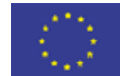 \\ europa.eu \\ STUDIARE IN ITALIA Intercultura e inclusione all'Università
}

a cura di Alessandro Vaccarelli

\section{La melagrana}

Ricerche e progetti per l'intercultura

FrancoAngeli OPEN 2 ACCESS 


\section{Collana diretta da Graziella Favaro e Massimiliano Fiorucci}

La collana La melagrana articola la sua proposta editoriale su due diversi piani dell' educazione interculturale: le idee e le pratiche.

La sezione Idee e metodi propone contributi teorici, riflessioni

e materiali che offrono spunti da sviluppare nel lavoro interculturale.

La sezione Ricerche e progetti descrive e commenta esperienze

e progetti realizzati, con uno sguardo attento al significato generale che possono avere anche in situazioni diverse da quelle in cui sono nati.

In ogni caso l'attenzione è rivolta a proporre dei testi che mettano in luce temi e problemi sinora poco sviluppati nell'ambito della pubblicistica sull'educazione

interculturale e che sappiano integrare i due piani che abbiamo indicato.

I lettori a cui è dedicata questa collana sono soprattutto gli insegnanti in formazione $o$ in servizio, ma i testi si rivolgono anche agli operatori dei servizi sociali, alle educatrici degli asili nido, alle figure di mediazione interculturale che non svolgono il loro lavoro nella scuola.

Questo anche nella convinzione che un efficace lavoro interculturale possa svilupparsi solo attraverso la collaborazione tra la scuola e le istituzioni

formative del territorio e con un contatto tra tutte le figure professionali che operano nei diversi ambiti.

\section{Comitato scientifico}

Ivana Bolognesi, Università di Bologna Marco Catarci, Università di Roma Tre Cristina Allemann-Ghionda, Università di Colonia Elio Gilberto Bettinelli, Università di Milano-Bicocca Giovanna Campani, Università di Firenze Don Virginio Colmegna, Fondazione Casa della Carità

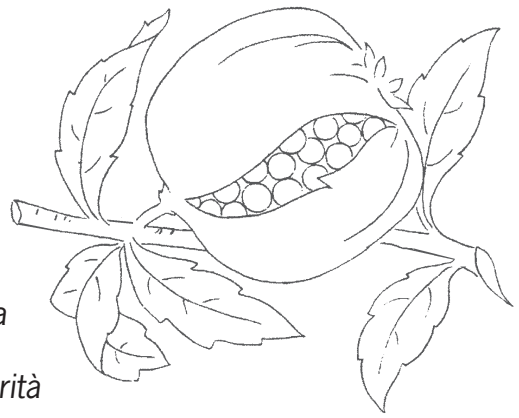
Duccio Demetrio, Università di Milano-Bicocca

F. Javier García Castaño, Università di Granada Antonio Genovese, Università di Bologna Francesca Gobbo, Università di Torino Jahdish Gundara, Università di Londra Lorenzo Luatti, Ucodep - Centro di Documentazione Città di Arezzo

Raffaele Mantegazza, Università di Milano-Bicocca

Giuseppe Milan, Università di Padova

Marie Rose Moro, Università di Paris Descartes

Vinicio Ongini, esperto Miur

Agostino Portera, Università di Verona

Milena Santerini, Università Cattolica del Sacro Cuore, Milano

Clara Silva, Università di Firenze

Massimiliano Tarozzi, Università di Bologna

Maria Sebastiana Tomarchio, Università di Catania

Alessandro Vaccarelli, Università dell'Aquila

Davide Zoletto, Università di Udine

Tutti i volumi pubblicati sono sottoposti a referaggio in "doppio cieco". Il Comitato scientifico può svolgere anche le funzioni di Comitato dei referee. 



\section{STUDIARE IN ITALIA Intercultura e inclusione all'Università}

a cura di Alessandro Vaccarelli

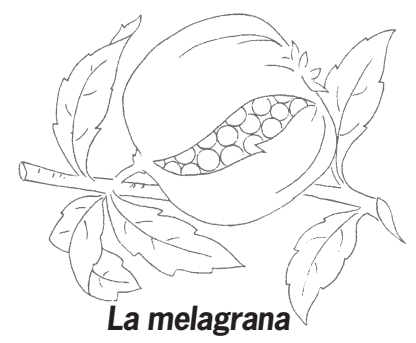

Ricerche e progetti per l'intercultura

\section{FrancoAngeli}




\section{Progetto cofinanziato da}

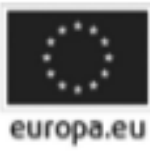

Il presente volume viene distribuito e reso accessibile in forma gratuita poiché finanziato con il contributo del FEI (Fondo Europeo per l'Integrazione dei cittadini di Paesi terzi), progetto “Unidiversità”, annualità 2013, Azione 7, PROG-1059381.

L'opera, comprese tutte le sue parti, è tutelata dalla legge sul diritto d'autore ed è pubblicata in versione digitale con licenza Creative Commons Attribuzione-Non Commerciale-Non opere derivate 3.0 Italia (CC-BY-NC-ND 3.0 IT)

L'Utente nel momento in cui effettua il download dell'opera accetta tutte le condizioni della licenza d'uso dell'opera previste e comunicate sul sito http://creativecommons.org/licenses/by-nc-nd/3.0/it/legalcode 


\section{Indice}

Ringraziamenti

pag. 9

Presentazione, di Paola Inverardi

Prefazione, di Massimiliano Fiorucci

1. Introduzione: il progetto "Unidiversità", di Mario Alaggio

1.1. L'Aquila: una città multiculturale dopo un terremoto

1.2. Il progetto "Unidiversità": convivenza e intercultura in città e nelle aule accademiche

\section{Parte I}

Studiare in Italia: scenari e prospettive

2. Le migrazioni internazionali come bene comune, di Luigi

2.1. Sponda sud del Mediterraneo e pressione migratoria

2.2. L'Italia tra Europa e Africa: una geografia dei flussi

2.3. Gli immigrati nel Belpaese ai tempi della crisi

2.4. Figli della mobilità: un territorio per le seconde generazioni

3. Il tutore della convivenza e la progettualità interculturale nei contesti urbani e formativi, di Sergio Bontempelli

3.1. La "città multiculturale" tra realtà e mito

3.2. Differenze culturali e asimmetrie di potere

3.3. Mediare tra diseguali: l'esperienza di RicostruireInsieme nel territorio dell'Aquila 
3.4. Il tutore della convivenza e il rapporto tra università e città

pag. 48

4. L'università nel processo di internazionalizzazione e mobilità globale: obiettivi, risultati e sfide, di Anna Tozzi 4.1. Il sistema Italia di fronte alle sfide europee e globali » 50

5. I tempi sono maturi: intercultura all'università, di Alessandro Vaccarelli

5.1. Lo specchio deformante: il paradosso multiculturale della presenza straniera all'università

5.2. L'universo composito degli studenti di cittadinanza non italiana: studenti internazionali e studenti stranieri scolarizzati in Italia

5.3. Avanguardie migranti: i figli delle migrazioni all'università

5.4. Il sistema a imbuto: i passaggi dalla scuola all'università

5.5. I tempi sono maturi: intercultura all'università

74

75

\section{Parte II}

Studenti italiani e studenti di altra cittadinanza allo specchio: la ricerca e l'analisi dei dati

6. Una ricerca sugli studenti dell'Università dell'Aquila: studenti stranieri, studenti internazionali, studenti italiani, di Alessandro Vaccarelli

6.1. Obiettivi, strumenti e metodologie

6.2. I campioni studiati

6.3. Guida alla lettura dei dati

81

81

83

85

6.4. Il doppio binario della presenza straniera all'università: studenti stranieri scolarizzati in Italia e studenti internazionali. Alcune anticipazioni sui dati

86

7. Gli studenti di cittadinanza non italiana all'Università dell'Aquila in relazione al contesto nazionale, di Serena Castellani

7.1. Gli studenti di altra cittadinanza in Italia negli ultimi 10 anni

7.2. La distribuzione su scala regionale

7.3. La distribuzione per atenei italiani

7.4. Gli studenti di cittadinanza non italiana verso l'Università dell'Aquila negli ultimi 10 anni

\section{0} 0 
8. Gli studenti italiani e non: chi sono, di Francesco Marola, Sonia Pagnanelli

8.1. Introduzione

8.2. Il sesso e l'età

8.3. La provenienza geografica

8.4. Provincia di residenza e condizione abitativa

8.5 Le pratiche religiose

pag. 101

» 101

» 101

$\gg 102$

$\gg 104$

$\gg \quad 105$

8.6. Gli studenti di cittadinanza non italiana: studenti internazionali e studenti scolarizzati in Italia

$\gg \quad 108$

9. La formazione scolastica e universitaria: i percorsi, gli esiti, la motivazione allo studio, di Valentina Ciaccio

9.1. La formazione scolastica nei due campioni

9.2. La scelta del percorso universitario

9.3. Requisiti di accesso richiesti e corsi integrativi erogati

9.4. Provenienze ed esiti dei percorsi formativi

9.5. Le motivazioni nel percorso di studi universitari

9.6. Proposte di intervento

10. Studiare all'Università dell'Aquila: qualità di servizi e attrattività, di Arianna Fiorenza, Ilaria Salvati

10.1. La scelta della sede universitaria per gli studenti italiani e di altra cittadinanza

10.2. La percezione della qualità della formazione

10.3. La valutazione delle attività a favore degli studenti di cittadinanza non italiana

11. Alcuni aspetti della socialità in ambito universitario, di Arianna Fiorenza, Chiara Ciccozzi

11.1. Introduzione

11.2. Le relazioni all'interno dell'ambiente universitario

11.3. Natura delle relazioni e situazione sentimentale

11.4. Le esperienze di associazionismo

12. Chi è l'altro? Percezioni a confronto, di Maria Grazia Ferretti, Roberto Manzi

12.1. La percezione dell'altro

12.2. Le percezioni sull'immigrazione nello scenario nazionale e internazionale

12.3. Atteggiamenti e percezioni nell'Università dell'Aquila 
13. Pregiudizi e socialità in ambito universitario, di Alessandro Vaccarelli

13.1. Il pregiudizio e i "contesti" della ricerca

13.2. La presenza del pregiudizio secondo gli studenti italiani e di altra cittadinanza

13.3. Il pregiudizio tra gli studenti internazionali e gli studenti stranieri scolarizzati in Italia e la relazione con altre variabili

13.4. Pregiudizi, socialità, atteggiamenti nei confronti degli italiani

13.5. Oltre il pregiudizio: il bisogno di mediazione e le possibili azioni

14. Tempi e spazi per lo studio e per la vita sociale, di Marta Allevi, Francesca Palma

14.1. Il contesto socio-territoriale della città dell'Aquila

14.2. I flussi degli studenti

14.3. L'agire territoriale degli studenti: dove si muovono in città 14.4. L'esperienza degli studenti: la città e l'università

\section{Parte III \\ Le azioni positive e lo sguardo al futuro}

15. La progettazione partecipata: il gruppo come strumento per promuovere la convivenza, di Maria Marronaro, Farnaz Mirzapoor, Jean Pierre Ndayambaje 》 185

15.1. Introduzione 》 185

15.2. La formazione del gruppo dei tutori della convivenza » 188

15.3. Il gruppo come risorsa $\gg 189$

15.4. Gruppo ed emozioni nella formazione dei tutori della convivenza

15.5. Chi è il tutore della convivenza?

15.6. I tavoli di progettazione partecipata » 195

\section{Allegati}

I questionari per gli studenti italiani e di altra cittadinanza, di Maria Grazia Ferretti, Alessandro Vaccarelli » 201

Bibliografia 》 225

Gli autori $\gg 232$ 


\section{Ringraziamenti}

Si desidera ringraziare: Lina Calandra e il Laboratorio di Cartografia "Cartolab" del Dipartimento di Scienze Umane dell'Università dell'Aquila per i continui e proficui confronti di carattere interdisciplinare; Marco De Luca, Stefania Ciocca, Francesca Paoni dell'Ufficio Fundraising e gestione progetti di ateneo, il cui lavoro, svolto con grandissima professionalità, è stato fondamentale nella realizzazione del volume e dell'intero progetto "Unidiversità"; Ciro Marziliano dell'Ufficio statistico di ateneo, per il prezioso aiuto nella costruzione dei campioni raggiunti nel corso della ricerca; Serena Castellani, Chiara Ciccozzi e Arianna Fiorenza, per il supporto nel lavoro di revisione del testo, Arianna Apicella e Antonio Marangiolo per il supporto tecnologico.

Si ringraziano inoltre: gli operatori di banca dati e i tutori della convivenza coinvolti nel progetto, per il lavoro svolto con spirito di gruppo e con passione civile e scientifica: Davie Chuwa, Emblia Nazhai, Valentina Ciaccio, Arianna Fiorenza, Roberto Manzi, Francesco Marola, Oscarine Mbouna, Sonia Pagnanelli, Francesca Palma, Ilaria Salvati; Marta Allevi, Chafiq Azizi, Francesca Battella, Marianne Fideliz Bermudez, Barbara Bologna, Proches Joseph Chuwa, Guy El Khoury, Samira Farda, Ghirmai Abiel Ghebre, Peter Linson, Adinani Awadh Mushi, Fadi Obeid, Marco Pasqua, Romina Pendolino, Fabrizio Rea, Samuel Champlain Takam, Valeria Tiganik, Federica Tomassoni, Olena Yakymets; il gruppo Educare in movimento che ha attivato le sue energie in modo volontario e convinto, supportando nelle diverse fasi della ricerca e di elaborazione del volume. 



\title{
Presentazione
}

\author{
di Paola Inverardi*
}

Il progetto "Unidiversità", finanziato dal Ministero dell'Interno nell'ambito del Fondo Europeo per l'Integrazione di cittadini di Paesi terzi (FEI annualità 2013), rappresenta per l'Università dell'Aquila l'ambizioso tentativo scientificamente orientato, e dunque monitorato nei suoi passaggi e nei suoi esiti, di rispondere ai bisogni sociali e formativi dei suoi studenti, di affrontare il rapporto con il territorio e con il cambiamento sociale, di promuovere inclusione tanto nelle aule accademiche quanto nel tessuto urbano. Tutto ciò in una città come L'Aquila, che porta ancora i segni profondi del sisma 2009, e nella quale, accanto ad una ricostruzione materiale ancora presente per i prossimi anni, è forte l'esigenza anche di una ricostruzione sociale, culturale, educativa.

Il partenariato con il mondo dell'associazionismo, e in particolare con l'ARCI e con il Coordinamento RicostruireInsieme, ha consentito di basare il progetto sulla costituzione e sul consolidamento di quelle reti di rapporti e di competenze che in un territorio, nel nostro caso post-emergenziale e al tempo stesso multiculturale, dovrebbero sempre interagire. "Unidiversità" ha raggiunto centinaia di studenti attraverso una ricerca rigorosa e originale, ha messo a fuoco i limiti e le potenzialità del sistema universitario sul tema dell'inclusione degli studenti di cittadinanza non italiana, ha sondato nelle pieghe dei rapporti sociali tra gli attori in gioco, ha permesso di prevedere, a partire dai risultati ottenuti, possibili azioni future; ed ha animato la città e l'università, creando punti di incontro, iniziative di dialogo e scambio interculturale, tavoli di partecipazione, oltre ad aver promosso nuove professionalità sociali orientate alla convivenza e alla mediazione dei conflitti.

Il volume che ho il piacere di presentare documenta un percorso, di ricerca e di intervento, che apre la strada a numerose ulteriori iniziative. So-

* Rettrice dell'Università degli Studi dell'Aquila. 
prattutto segnala l'importanza di una nuova sensibilità che la cultura organizzativa e didattica accademica non può che iniziare ad assumere, se è vero che oggi l'universo degli studenti di altra cittadinanza viene profondamente a modificarsi rispetto all'idea tradizionale di studente internazionale; l'affacciarsi, lento ma progressivo, delle ragazze e dei ragazzi che riconosciamo come "figli" delle migrazioni, ci dà l'occasione, ancora una volta, di riflettere sui grandi temi che rimandano ad una visione di università aperta e democratica dove l'equità, l'orientamento e il successo formativo, il contrasto ai fattori di ostacolo al raggiungimento dei traguardi, le azioni di continuità con la scuola e con il territorio, la valorizzazione della diversità e l'avversione alle diseguaglianze siano prassi comune e partecipata. Il volume mette allo scoperto anche problemi di convivenza, momenti di criticità nei rapporti tra soggetti di culture diverse, limiti che un'istituzione seria non può che rilevare per affrontarli. Il nostro impegno, a conclusione del progetto, non può che essere dunque quello di sedimentare la conoscenza e l'esperienza e di assumere la responsabilità di portare avanti un'ottica interculturale dentro e fuori le aule accademiche, dentro i processi gestionali e didattici e soprattutto nelle interazioni che giorno per giorno ci qualificano nel nostro ruolo sociale e civile. 


\title{
Prefazione
}

\author{
di Massimiliano Fiorucci
}

L'Italia si presenta, nel sistema migratorio internazionale, con una storia migratoria del tutto particolare. Le grandi migrazioni del secolo scorso e quelle in atto, infatti, hanno coinvolto e continuano a coinvolgere il nostro Paese in una triplice prospettiva: dapprima come terra di emigrazione (quasi 30 milioni di espatriati dall'Unità d'Italia ad oggi; oltre 60 milioni di oriundi italiani nel mondo e, attualmente, 4.482.115 italiani residenti all'estero iscritti all'Anagrafe degli Italiani Residenti all'Estero - AIRE al $1^{\circ}$ gennaio 2014) (Fondazione Migrantes, 2014), successivamente come Paese di immigrazione (il 1973 è l'anno in cui si è registrato, per la prima volta, un "saldo migratorio" positivo") e parallelamente come teatro di intensi spostamenti di popolazione interni.

Attualmente l'Italia è, dunque, sia un Paese di emigrazione sia un Paese di immigrazione: in conseguenza di ciò appare più corretto e più coerente parlare di "migrazioni". Tale nozione consente di non dimenticare e di non trascurare la lunga vicenda delle "migrazioni interne" che oscilla tra fasi di rimozione e momenti di centralità mediatica. Le migrazioni interne hanno avuto, invece, un ruolo chiave nella storia italiana (Ginsborg, 1989; Bonifazi, 2013) e sono strettamente legate ai cambiamenti sia economici che culturali e socio-demografici avvenuti nel Paese. Secondo lo storico Paul Ginsborg nel «ventennio 1951-71 la distribuzione geografica della popolazione italiana subì uno sconvolgimento. L'emigrazione più massiccia ebbe luogo tra il 1955 e il 1963; la tendenza migratoria si bloccò brevemente a metà degli anni '60, ma riprese poi fortemente negli anni 1967-71. In tutto, fra il 1955 e il 1971, 9.140.000 italiani sono coinvolti in migrazioni interregionali» (Ginsborg, 1989).

1. Con tale terminologia demografica si intende dire che le persone che sono entrate nel Paese (immigrati) sono numericamente maggiori rispetto a quelle che sono uscite (emigrati). Questo anno segna simbolicamente il passaggio dell'Italia da terra di emigrazione a terra di immigrazione. 
Non è più possibile, come mostrano con evidenza i dati storici e demografici, trincerarsi dietro la falsa e comoda rappresentazione dell'Italia come di un Paese che sarebbe impreparato ad affrontare la gestione dei fenomeni migratori poiché solo da poco a contatto diretto con essi: solo a voler considerare la questione dal punto di vista dell'immigrazione straniera in Italia sono passati comunque più di 40 anni dal suo avvio. A tale proposito va precisato che alla fine del 2013 gli stranieri residenti nel Paese erano ufficialmente 4.922.085 su una popolazione complessiva di 60.782.668, ma il Centro Studi e Ricerche IDOS stima una presenza effettiva di 5.364.000 persone in posizione regolare (IDOS, 2014). Le donne rappresentano il $52,7 \%$ del totale, i minori oltre 1 milione (925.569 quelli con cittadinanza non comunitaria) e 802.785 gli iscritti a scuola con cittadinanza non italiana nell'a.s. 2013/14 (il 9,0\% della popolazione scolastica complessiva). L'incidenza dei residenti stranieri sulla popolazione totale ha raggiunto, invece, l'8,1\% (1 ogni 12 abitanti) e la distribuzione territoriale della popolazione straniera residente in Italia risulta essere la seguente: Nord 60,1\%, Centro $25,4 \%$, Sud $14,6 \%$.

Per quanto concerne le aree di origine dei migranti va segnalato che la maggior parte di loro proviene dall'Europa $(52,8 \%)$, seguono in ordine decrescente Africa (20,9\%), Asia (18,3\%) e America (7,9\%). L'estrema eterogeneità dei Paesi di provenienza (circa 190) e l'alto numero di nazionalità rilevate sul territorio hanno portato alcuni studiosi a definire la società italiana come una sorta di "arcipelago migratorio", in quanto sono presenti, con percentuali diverse, persone provenienti da quasi tutti i Paesi del mondo. E, tuttavia, i primi cinque Paesi per provenienza sono la Romania (933mila presenze), il Marocco (525mila), l'Albania (503mila), la Cina (321mila) e l'Ucraina (234mila) i cui cittadini rappresentano quasi la metà dei migranti presenti in Italia (Ivi).

Questo flusso migratorio ha avuto forti ripercussioni sul sistema scolastico, in poco più di quindici anni il numero di studenti stranieri si è più che decuplicato, passando da 59.389 unità (a.s. 1996/97) a 802.844.630 (a.s. 2013/14) con un'incidenza percentuale di bambini e ragazzi di cittadinanza estera che ha raggiunto il 9\% (MIUR e ISMU, 2015). «Se si tiene conto che i Paesi di provenienza sono circa 200, è facile intuire la complessità del fenomeno soprattutto quando si tratta di sviluppare iniziative volte al sostegno dell'integrazione di alunni stranieri» (MIUR, 2012).

Di fronte ad una tale situazione è necessario elaborare risposte all'altezza dei problemi del presente. I processi di globalizzazione in atto e la configurazione in senso multiculturale delle società interrogano in profondità i sistemi educativi e formativi, che devono mirare ad una prospettiva di mediazione interculturale. La formazione degli insegnanti, degli educatori e degli operatori occupa, in tale contesto, un posto di tutto rilievo: è solo 
a partire da una corretta impostazione interculturale del lavoro socio-educativo nella scuola e nella società che si può sperare di diffondere una sempre più necessaria "cultura della convivenza". Non si tratta di un obiettivo facile: educatori ed operatori sono chiamati per primi a rimettere in discussione i propri paradigmi di riferimento con l'obiettivo di attenuare l'alto tasso di etnocentrismo presente nel sistema educativo e formativo.

La relazione tra immigrazione e formazione rappresenta un tema cruciale che comporterà anche per l'università una profonda opera di revisione, di rivisitazione e di rifondazione dell'asse formativo che deve mirare alla formazione di un cittadino del mondo, che vive e agisce in un mondo interdipendente.

La ricerca dell'Università dell'Aquila, coordinata da Alessandro Vaccarelli, si colloca all'interno dei filoni di ricerca della pedagogia interculturale che per sua natura dialoga con le altre scienze umane e deve necessariamente inverarsi nell'intervento e nella pratica. La ricerca che il libro presenta costituisce il primo studio sistematico di carattere pedagogico-interculturale condotto sugli studenti universitari di cittadinanza non italiana $\mathrm{e}$, in generale, uno dei primi lavori nello scenario nazionale, con un precedente, di taglio sociologico e nella prospettiva della metodologia qualitativa, condotto nell'Università di Genova (Lagomarsino e Ravecca, 2014).

Nella consapevolezza di quanto la pedagogia interculturale più matura afferma ormai da anni, il volume considera l'universo degli studenti di cittadinanza non italiana nelle sue diverse connotazioni, come mondo di per sé composito e articolato, internamente variabile: la prima grande distinzione tra la figura più classica dello studente internazionale e lo studente straniero scolarizzato in Italia (i figli delle migrazioni) già consente di orientare non solo la futura ricerca, ma anche le politiche formative che inevitabilmente dovranno essere pensate anche per il mondo accademico. E, dentro questa prima grande distinzione, attraverso un'analisi dei dati statistici che ha incrociato numerose variabili, si legge un ulteriore distinguersi di situazioni e condizioni che chiamano in causa le nazionalità, le variabili di natura socio-economica, i fattori religiosi e culturali, i motivi e i "tempi" della presenza in Italia, ecc. Si coglie, inoltre, nell'idea dello "specchio", vale a dire nello studio della componente studentesca di cittadinanza italiana e del confronto con quella di altre cittadinanze, un approccio ancora una volta maturo alla ricerca pedagogico-interculturale: a pesare sul successo formativo non troviamo solo fattori legati alle condizioni socio-economiche o alla conoscenza della lingua italiana, ma anche quelli, esplorati in modo piuttosto approfondito, che rimandano agli atteggiamenti reciproci, al mondo delle relazioni, alla presenza di pregiudizi e di fattori di discriminazione che sono attivi anche in ambito accademico. L'inclusione in questo senso, come anche dimostrato dalle prassi che il libro 
documenta, non può essere soltanto il fine degli interventi, ma anche la condizione imprescindibile, il "mezzo" dunque, per raggiungere il successo formativo. Il confronto con il mondo della scuola, i dati che vengono proposti e analizzati, le riflessioni su un sistema formativo definito "a imbuto" aprono problemi e prospettive che rimandano ai temi dell'equità dell'istruzione e che non possono che essere letti nell'ottica della continuità formativa.

Come segnala una ricerca dell'European Migration Network, in Italia la presenza di studenti stranieri nelle università resta ancora di molto inferiore rispetto a quella registrata in molti altri Paesi europei e, tuttavia, è destinata a crescere nei prossimi anni: in Gran Bretagna vi sono 550mila studenti universitari con cittadinanza non nazionale, in Germania e Francia 250mila, mentre in Italia solo 70mila (nel nostro Paese l'incidenza degli stranieri sul totale della popolazione passa dal $4,5 \%$ tra gli immatricolati, al 3,8\% tra gli iscritti complessivi all'università, fino al 2,7\% tra i laureati) (Ministero dell'Interno e IDOS, 2013). La presenza di studenti universitari di origine straniera rappresenta per l'Italia una sfida e l'accesso delle cosiddette "seconde generazioni" all'università costituisce un indicatore del livello di democrazia di una società. Se la società italiana sarà capace di garantire alle seconde e terze generazioni una "integrazione non subalterna", se anche ai cittadini italiani di origine immigrata sarà offerta la possibilità di diventare insegnanti, medici, avvocati, ingegneri, ecc. forse si potrà parlare a giusto titolo della nostra come di una società equa. 


\title{
1. Introduzione: il progetto "Unidiversità"
}

\author{
di Mario Alaggio
}

\subsection{L'Aquila: una città multiculturale dopo un terremoto}

Dal terremoto del 2009, più ancora che dal tempo, il territorio dell'Aquila e del suo circondario è segnato dallo spazio; dallo spazio negato alla socialità, che in una città terremotata significa l'assenza del centro storico. Dagli spazi delle periferie e dei borghi del cratere sismico, nei quali la ricostruzione ancora non parte, ridotti ai nuovi insediamenti abitativi, con acronimi a volte ammiccanti come CASE (Complessi Antisismici Sostenibili ed Ecocompatibili), a volte meno, MAP (Moduli Abitativi Provvisori). Il nostro territorio è segnato dalla mancanza di spazi sociali e di relazione, di incontro fine a se stesso, alla conoscenza dell'altro.

Che la convivenza con l'altro qui fosse in gioco, più che altrove, ce ne siamo accorti già dalle tendopoli, strutture di accoglienza per la popolazione terremotata, dove l'accesso agli stranieri era reso difficoltoso, dove tutti gli stereotipi razzisti e xenofobi trovavano spazio tra le paure e la rabbia. Ma la completa assenza di punti di riferimento che si prova dopo un terremoto che stravolge la quotidianità, i ruoli, le autocensure, è stata una novità inebriante; scintilla della rinascita collettiva potenzialmente diversa dallo status precedente.

Così accade che il nostro territorio, dal 2009, è percorso da esperienze sociali nuove, dai primi comitati di cittadini e cittadine che vogliono essere partecipi delle decisioni sulla ricostruzione fisica e sociale della città fino alle iniziative più disparate, dal teatro alla tutela dell'ambiente, dall'intercultura alla fotografia.

C'è una nuova progettualità che corre libera nella mente e nei cuori delle persone; è alimentata dalla generosità delle volontarie e dei volontari che da tutta Italia portano energie e idee. E allora diventa facile sognare di poter ricostruire una città a misura di tutti e tutte, che sia arricchita e non impaurita dalle differenze che portano i nuovi cittadini e le nuo- 
ve cittadine straniere, che non abbia paura, nonostante tutto, di accogliere chi ha bisogno.

E così il lavoro che prima raramente era svolto insieme da organismi e associazioni per la tutela sociale e la promozione del dialogo interculturale diviene sinergico: appare evidente che solo coinvolgendo le associazioni che a vario titolo si occupano di immigrati e lavorando in stretto raccordo, si può ricostruire insieme il tessuto sociale in un territorio devastato e nel quale cominciano ad affiorare preoccupanti segnali di tensione che rimarcano le difficoltà di convivenza tra immigrati e italiani e tra italiani stessi. L'ARCI provinciale dell'Aquila, la Caritas Diocesana dell'Aquila, il Patronato SIAS, le associazioni Rindertimi di Avezzano, Gentium, Asil, Afipo, Unimondo, IntiRaymi, l'Iris di Teramo, la Cooperativa Pralipé di Pescara si riuniscono in un unico coordinamento, RicostruireInsieme.

Nel tempo il coordinamento cambia composizione, inevitabilmente ci sono degli avvicendamenti, ma la sinergia dà presto i suoi frutti. Con l'aiuto del Centro informativo per l'immigrazione (CINFORMI) dell'assessorato alla solidarietà internazionale e alla convivenza della provincia autonoma di Trento si attiva una progettazione che viene accolta dal Ministero dell'Interno (FEI) e consente di condurre una ricerca socio-demografica sul contesto territoriale: vogliamo misurare lo stato di salute della convivenza.

Nel luglio del 2010 si realizza la ricerca Immigrati e italiani dopo il terremoto nel territorio aquilano. Ricerca sui bisogni sociali, educativi e sullo stato della convivenza (Vaccarelli, 2010; 2012)1. Ne emergono dati di estremo interesse a riprova dell'estrema vulnerabilità, abitativa, lavorativa e relazionale degli stranieri residenti, ma ancor più la ricerca ci dà delle informazioni sul punto di vista degli italiani: più della metà di loro vorrebbe dare agli italiani, ad appena un anno dal sisma e con la distribuzione degli alloggi in atto, la priorità nella assegnazione di un'abitazione provvisoria; inoltre, alla richiesta di quantificare, in percentuale, la presenza degli stranieri sul territorio aquilano, gli intervistati italiani danno risposte che evidenziano una percezione assai distorta, infatti solo un terzo di essi si avvicina al dato reale (circa $6 \%$ ), mentre gli altri indicano percentuali assai superiori (tra il 10 e il 25\%, o addirittura superiore al 30\%). Da notare, ancora, che il $19 \%$ degli intervistati stranieri dichiara di essere stato vittima di episodi di intolleranza/razzismo. Questi ultimi dati si allineano con una situazione nazionale, ma assumono un risalto assai maggiore per il loro carico di potenzialità sociali negative all'interno di un contesto terremotato, in cui abitazioni, lavoro, degrado costituiscono i temi caldi delle presenti e future scommesse sociali e politiche. Il senso di frustrazione può favorire

1. www.ricostruireinsieme.it/2010/07/10/la-convivenza-nel-dopo-terremoto-la-ricercadi-ricostruire-insieme/. 
forme di discriminazione o di conflittualità con gli immigrati, deteriorando anche la coesione sociale più complessiva.

Emerge, dunque, la necessità di costruire reti sociali significative, che svolgano funzioni di contenimento sociale e psicologico, di solidarietà tra pari, ecc.; bisogni che riguardano la sfera dell'esistenza e della resilienza a più livelli e la qualità della convivenza.

Una nuova progettualità viene messa in campo, questa volta in Piazza d'Arti, nuova piazza sorta nel territorio urbano aquilano dall'iniziativa di 16 associazioni che hanno deciso di riunirsi appunto intorno ad una piazza, luogo naturale dell'incontro e della relazione, dove potenzialmente superare vecchie barriere e stereotipi.

\subsection{II progetto "Unidiversità": convivenza e intercultura in città e nelle aule accademiche}

A partire da questo contesto, l'Università dell'Aquila in partenariato con le associazioni RicostruireInsieme e Comitato Territoriale ARCI dell'Aquila, attive da anni in iniziative interculturali, e con il sostegno dell'Associazione Bibliobus, ha avviato le attività del progetto denominato "Unidiversità" finanziato dal Ministero dell'Interno nell'ambito del Fondo Europeo per l'Integrazione di cittadini di Paesi terzi (FEI - annualità 2013).

Il Progetto ha inteso valorizzare l'alta presenza di studenti di cittadinanza non italiana attraverso l'attivazione di tavoli di progettazione partecipata aperti agli studenti e alle studentesse dell'Aquila che, partendo dall'analisi dei bisogni espressi dalla comunità studentesca aquilana, hanno di fatto elaborato alcune proposte di intervento nel contesto universitario e cittadino in tema di convivenza e di inclusione, cercando di superare i conflitti imputabili alle differenze culturali, nel rispetto dei valori e degli stili di vita di ciascuno.

Si è cercato dunque di rispondere all'esigenza sempre più pressante di intervenire sui conflitti, potenziali o già in atto, attraverso la formazione di tutori della convivenza, vale a dire di ragazzi e ragazze, stranieri ed italiani, che hanno operato e che potranno operare per favorire un rapporto positivo fra soggetti di culture diverse, stabilendo relazioni di incontro e di scoperta, oltrepassando i confini di senso propri di ogni identità. La figura del tutore della convivenza non si sovrappone a quella, già esistente, del mediatore linguistico-culturale; pur avendo dei tratti comuni (la mediazione dei conflitti, il sostegno all'inserimento sociale, la facilitazione nelle relazioni interculturali ecc.), agisce in un ambito più ampio. In altre parole, 
a differenza del mediatore linguistico-culturale, il tutore della convivenza dovrebbe intervenire non all'interno di uno specifico servizio o di una specifica struttura (in ospedale, a scuola), ma nell'ambito territoriale e di relazioni definito da una città, da un contesto urbano, sociale, comunitario o di quartiere. Si tratta dunque di un intervento che è a un tempo più ampio e più indeterminato, prezioso soprattutto in un contesto come quello descritto, dove si rende particolarmente urgente un'opera di mediazione sociale su scala comunitaria, per ricostruire tessuti di relazioni e forme di convivenza spesso lacerate dagli effetti (sociali, prima che fisici) del sisma, che operi per il superamento delle visioni stereotipate.

In quello che è stato definito "il più grande cantiere d'Europa" la rappresentazione sociale degli immigrati (forza lavoro, ma diversa e percepita come potenzialmente pericolosa) rende difficile la comunicazione tra cittadini autoctoni e non, per cui il rapporto tra culture diverse che entrano in relazione avviene in maniera asimmetrica; si costruiscono così, con i tutori della convivenza, spazi fisici e simbolici, in Piazza d'Arti, per far emergere la ricchezza culturale delle comunità dei migranti maggiormente rappresentative cercando di decostruire visioni stereotipate e pregiudiziali che costituiscono non certamente l'unico, ma quanto meno il principale ostacolo al processo di integrazione.

Si è cercato inoltre, ad onor del vero finora senza successo, di coinvolgere i cittadini immigrati sia nella progettazione che nella gestione concreta delle iniziative che li riguardano, attraverso la costituzione di forme associative interculturali. Nel territorio della provincia dell'Aquila i cittadini immigrati generalmente si riuniscono e si riconoscono in gruppi di appartenenza nazionale e monoculturale che hanno come referente un soggetto socialmente riconosciuto da tutti i membri; le poche associazioni, perlopiù anch'esse monoculturali, hanno spesso vita breve e conflittuale e non contemplano la partecipazione di cittadini italiani.

A fronte di questo insuccesso, più o meno nello stesso periodo si è avviata una esperienza di accoglienza fondamentale per l'identità nuova, solidale ed interculturale, che vogliamo costruire per questa città: L'Aquila si è aperta ai rifugiati e ai richiedenti asilo politico. La città, con le sue difficoltà e le sue ferite, ha accolto chi fugge dalla disperazione e dalla guerra come forse non avrebbe fatto prima del sisma.

Dal 2012 il comune dell'Aquila, avvalendosi delle competenze del Comitato Territoriale ARCI dell'Aquila, ha accolto, entrando nella rete del Servizio Protezione Richiedenti Asilo e Rifugiati (SPRAR) circa 70 persone, garantendo una serie di servizi quali l'assistenza sanitaria, l'assistenza sociale, attività interculturali, l'inserimento scolastico dei minori, la mediazione linguistica e interculturale, l'orientamento e l'informazione legale, servizi per l'alloggio, servizi per la formazione e l'inserimento lavorativo, 
non solo ai beneficiari del progetto ma anche ad un ampio numero di cittadini immigrati, collaborando con il privato sociale, con le scuole e con l'Università degli Studi dell'Aquila.

Finora, vittime anche noi forse della visione stereotipata dello straniero, non avevamo mai rivolto le nostre attenzioni alla popolazione universitaria straniera residente nella nostra città; eppure le studentesse e gli studenti stranieri, provenienti da più di 40 Paesi diversi, sono più di 500 pari a circa il 15\% delle presenze straniere nel comune dell'Aquila.

Il progetto "Unidiversità" si compone di 5 attività integrate, che verranno riportate nei loro esiti e nei loro contenuti all'interno del presente volume.

La prima è stata finalizzata alla creazione e alla gestione di una banca dati e al monitoraggio dello stato della convivenza. In questa azione sono state selezionate 10 persone con funzioni di operatori di banca dati, che hanno raggiunto per la somministrazione di un questionario più di 500 studenti italiani e di altra cittadinanza, supportando inoltre il lavoro di trattamento dei dati. Pertanto si è dato vita all'interno dell'ateneo ad un processo laboratoriale, gestito da operatori di banca dati e tutori della convivenza, da intendersi come momento di raccolta ed elaborazione di quelle informazioni da parte della comunità universitaria aquilana, sui maggiori disagi, le principali cause di incomprensione, le aspettative di entrambe le parti, ecc.

Da ciò è emersa una ricerca che ha voluto mettere allo specchio i bisogni, le aspettative, gli atteggiamenti, i percorsi accademici degli studenti italiani e di altra cittadinanza. La comunità migrante degli studenti e delle studentesse, come vedremo nella seconda parte del volume, pur rappresentando una comunità per certi versi percepita come privilegiata, evidenzia comunque, appena interrogata, problemi di integrazione sociale e culturale legati a fenomeni di emarginazione all'interno del contesto accademico e cittadino, o ancor peggio episodi di discriminazione nella ricerca di un alloggio o nello svolgimento di pratiche amministrative o addirittura esclusione dall'accesso ai servizi per la mancanza di informazioni e a causa della poca chiarezza delle procedure burocratico-amministrative. Sono studenti, inoltre, che non possono essere considerati come un universo omogeneo e dai tratti definiti, con differenze di condizione, di status, di competenze linguistiche in italiano che accompagnano l'altro grande fattore di diversificazione, vale a dire la provenienza geografica e culturale. La ricerca, condotta in una prospettiva pedagogica, con aperture interdisciplinari nei campi della geografia e della sociologia, ha messo in luce anche un'altra macro-distinzione che rende ancor più complessa la lettura del fenomeno e l'intervento sociale ed educativo: gli studenti di cittadinanza non italiana pongono domande e bisogni diversi quando considerati in quanto studenti internazionali (veri e propri migranti per motivi di studio) o studenti "figli delle migrazioni", vale a dire soggetti residenti in Italia già da 
prima del loro accesso all'università, scolarizzati nella scuola italiana e già conoscitori dei modelli sociali e culturali della società che li accoglie.

In parallelo alla costruzione della banca dati e della ricerca, al fine di attivare processi di facilitazione della convivenza e dell'incontro interculturale, in un'ottica di conoscenza, dialogo e apertura verso l'Altro, studenti universitari italiani e di Paesi differenti hanno partecipato ad un percorso di formazione per tutori della convivenza, per divenire punti di riferimento per il dialogo interculturale dentro il contesto accademico e quello cittadino. La formazione ha favorito un immediato confronto tra culture stabilendo relazioni di incontro e di scoperta, fornendo ai partecipanti degli strumenti necessari per operare in un ambito interculturale. Il corso di formazione, della durata di 30 ore e tenuto da esperti di rilevanza nazionale e internazionale del mondo accademico e del mondo del volontariato sociale, è stato destinato a 20 soggetti (10 studenti e 10 soggetti con esperienze nel campo del volontariato sociale) selezionate per poter acquisire le competenze e le conoscenze necessarie a svolgere le funzioni del tutore della convivenza.

L'azione saliente - forte dei risultati della ricerca e del percorso formativo - è consistita poi nell'attuare forme di progettazione partecipata: analizzando le informazioni della banca dati, tutrici e tutori della convivenza, in collaborazione con gli operatori del progetto e delle associazioni partner, hanno costituito tavoli di lavoro per attivare percorsi di progettazione partecipata in grado di valorizzare le ricchezze e le differenze culturali.

I gruppi si sono riuniti con cadenza settimanale sia all'interno dell'università sia presso la sede del Coordinamento RicostruireInsieme, in un luogo, Piazza d'Arti, ideale - anche per il significato simbolico che ha assunto nel dopo sisma - per riportare all'esterno dell'ambiente accademico, dunque verso la città, gli stimoli ad una progettazione partecipata che veda anche coinvolte le varie associazioni di rappresentanza delle comunità straniere presenti nel territorio. L'ultima parte del volume documenta in sintesi lo spirito, le finalità, gli approcci utilizzati nella formazione dei tutori e nella implementazione dei tavoli partecipati.

Un'ulteriore azione, legata ai tavoli di progettazione partecipata, è stata la realizzazione di un programma di iniziative multiculturali che hanno visto come destinatari sia gli studenti universitari sia l'intera cittadinanza. Tali iniziative (incontri con scrittori stranieri, percorsi artistici e sportivi, seminari di incontro ed approfondimento, cene interculturali) sono state realizzate in parte all'interno dell'università, in parte in contesti più informali ed allargati all'intera cittadinanza. Gli appuntamenti culturali hanno tratto spunto dalle riflessioni e dai dati rilevati e hanno cercato di "presentare" le ricchezze e le differenze culturali di tutte le comunità rappresentate. Le iniziative sono state realizzate in collaborazione con le associazioni dell'A- 
quila e con l'attivo sostegno delle associazioni di studenti stranieri coinvolte a più livelli nel progetto.

Consapevoli che anche la conoscenza di tradizioni, religioni, stili di vita di altri Paesi non può più essere caratterizzato da toni documentaristici o folkloristici, ma deve saper suscitare l'attenzione per influire sulle conoscenze e coscienze di tutti, è stato realizzato uno studio di fattibilità di una web-radio unidiversitaria capace di parlare il linguaggio corrente e raggiungere tutti nel modo appropriato.

Anche in questo progetto, e in tutti e tutte che lo rendono vivo, c'è un tratto comune: la convinzione che il miglioramento della conoscenza reciproca possa migliorare la convivenza tra nuovi cittadini e società ospitante. Perché non accada più che qualcuno - nello specifico un tutore della convivenza senegalese - debba dire di aver scoperto il razzismo in una città come L'Aquila. 

Parte I

Studiare in Italia: scenari e prospettive 



\title{
2. Le migrazioni internazionali come bene comune
}

\author{
di Luigi Gaffuri
}

\subsection{Sponda sud del Mediterraneo e pressione migratoria}

Secondo le proiezioni delle Nazioni Unite, i 7,2 miliardi d'individui dell'odierna popolazione mondiale sono destinati a diventare 8,1 nel 2025, 9,6 nel 2050 e quasi 11 miliardi sul finire del XXI secolo. Oltre la metà di questa crescita demografica, da oggi al 2050, si verificherà in Africa, dove gli abitanti passeranno dall'attuale miliardo a ben oltre il doppio (UN-DESA, $2013)^{1}$. Nel bel mezzo del secolo in cui viviamo, la più gran parte di tale popolazione sarà ospitata dalle sole città (nelle quali il carico demografico risulterà ampiamente superiore a quello complessivo odierno dell'intera Africa), la cui incidenza su quella totale del continente è oggi ancora inferiore alla media registrata nelle Nazioni industrialmente avanzate o alla media degli abitanti urbani negli altri Paesi in via di sviluppo (Tab. 1).

Se il mondo delle città accoglie ora poco più di 400 milioni di africani, nel 2050 sarà più che triplicata e ne ospiterà oltre un miliardo e 250 milioni, mentre la Nigeria avrà più abitanti degli Stati Uniti e, sul finire del secolo, rivaleggerà con la Cina per il secondo posto dopo l'India come Paese più popoloso del pianeta (UN-Habitat, 2014, pp. 262 ss.). Nel 2100 altre Nazioni africane, come la Tanzania, la Repubblica Democratica del Congo, l'Etiopia, l'Uganda e il Niger avranno oltre 200 milioni di abitan-

1. Conviene precisare che l'impostazione metodologica qui adottata, riguardo al fenomeno dell'immigrazione, non può prescindere dalla presa in carico della situazione nei territori di provenienza dei migranti, dove si originano, per un insieme di cause complesse e mutevoli tra le quali spiccano quelle economiche e demografiche, i flussi in uscita che appunto caratterizzano le dinamiche dell'emigrazione. Con le stesse parole di Sayad: «Non c'è immigrazione in un luogo senza che vi sia stata emigrazione da un altro luogo; non c'è presenza da qualche parte che non sia pagata da un'assenza in un'altra parte. La denominazione stessa di immigrato rinvia implicitamente a quella di emigrato, che ne è il corollario» (Sayad, 2008, p. 79). 
Tab. 1 - Popolazione africana nel 2010, proiezioni al 2050 (in migliaia)

\begin{tabular}{|c|c|c|c|c|c|c|}
\hline $\begin{array}{c}\text { Paesi per } \\
\text { grandi aree }\end{array}$ & $\begin{array}{c}2010 \\
\text { pop. totale }\end{array}$ & $\begin{array}{c}2050 \\
\text { pop. tot. }\end{array}$ & $\begin{array}{c}2010 \\
\text { pop. urb. }\end{array}$ & $\begin{array}{c}2050 \\
\text { pop. urb. }\end{array}$ & $\begin{array}{l}2010(\%) \\
\text { abit. città }\end{array}$ & $\begin{array}{l}2050(\%) \\
\text { abit. città }\end{array}$ \\
\hline Africa & 1.022 .234 & 2.191 .599 & 400.651 & 1.264 .629 & 39,2 & 57,7 \\
\hline Africa subsahariana & 822.724 & 1.891 .711 & 298.402 & 1.068 .752 & 36,3 & 56,5 \\
\hline Africa settentrionale & 199.511 & 299.888 & 102.249 & 195.877 & 51,2 & 65,3 \\
\hline Algeria & 35.468 & 46.522 & 25.546 & 40.649 & 72,0 & 87,4 \\
\hline Egitto & 81.121 & 123.452 & 35.186 & 74.040 & 43,4 & 60,0 \\
\hline Libia & 6.355 & 8.773 & 4.929 & 7.462 & 77,6 & 85,1 \\
\hline Marocco & 31.951 & 39.200 & 18.109 & 28.430 & 56,7 & 72,5 \\
\hline Sahara Occidentale & 531 & 901 & 434 & 802 & 81,8 & 89,0 \\
\hline Sudan & 33.604 & 68.391 & 11.117 & 34.731 & 33,1 & 50,8 \\
\hline Tunisia & 10.481 & 12.649 & 6.928 & 9.762 & 66,1 & 77,2 \\
\hline Africa occidentale & 304.261 & 743.850 & 134.810 & 488.886 & 44,3 & 65,7 \\
\hline Benin & 8.850 & 21.734 & 3.917 & 14.490 & 44,3 & 66,7 \\
\hline Burkina Faso & 16.469 & 46.721 & 4.227 & 25.801 & 25,7 & 55,2 \\
\hline Capo Verde & 496 & 632 & 307 & 502 & 61,8 & 79,5 \\
\hline Costa d'Avorio & 19.738 & 40.674 & 9.979 & 29.319 & 50,6 & 72,1 \\
\hline Gambia & 1.728 & 4.036 & 979 & 2.960 & 56,7 & 73,3 \\
\hline Ghana & 24.392 & 49.107 & 12.492 & 35.520 & 51,2 & 72,3 \\
\hline Guinea (Conakry) & 9.982 & 23.006 & 3.490 & 13.443 & 35,0 & 58,4 \\
\hline Guinea Bissau & 1.515 & 3.185 & 655 & 2.010 & 43,2 & 63,1 \\
\hline Liberia & 3.994 & 9.660 & 1.909 & 6.384 & 47,8 & 66,1 \\
\hline Mali & 15.370 & 42.130 & 5.268 & 24.937 & 34,3 & 59,2 \\
\hline Mauritania & 3.460 & 7.085 & 1.426 & 4.282 & 41,2 & 60,4 \\
\hline Niger & 15.512 & 55.435 & 2,733 & 20.546 & 17,6 & 37,1 \\
\hline Nigeria & 158.423 & 389.615 & 77.629 & 277.916 & 49,0 & 71,3 \\
\hline Senegal & 12.434 & 28.607 & 5.253 & 17.579 & 42,3 & 61,4 \\
\hline Sierra Leone & 5.868 & 11.088 & 2.281 & 6.599 & 38,9 & 59,5 \\
\hline Togo & 6.028 & 11.130 & 2.262 & 6.597 & 37,5 & 59,3 \\
\hline Africa orientale & 333.993 & 802.184 & 77.954 & 358.974 & 23,3 & 44,7 \\
\hline Burundi & 8.383 & 13.703 & 892 & 3.829 & 10,6 & 27,9 \\
\hline Comore & 735 & 1.700 & 206 & 682 & 28,0 & 40,1 \\
\hline Eritrea & 5.254 & 11.568 & 1.098 & 5.151 & 20,9 & 44,5 \\
\hline Etiopia & 82.950 & 145.187 & 13.900 & 51.601 & 16,8 & 35,5 \\
\hline Gibuti & 889 & 1.620 & 684 & 1.363 & 77,0 & 84,2 \\
\hline Kenya & 40.513 & 96.887 & 9.549 & 44.302 & 23,6 & 45,7 \\
\hline Madagascar & 20.714 & 53.561 & 6.614 & 30.640 & 31,9 & 57,2 \\
\hline Malawi & 14.901 & 49.719 & 2.316 & 15.670 & 15,5 & 31,5 \\
\hline Maurizio & 1.299 & 1.367 & 543 & 727 & 41,8 & 53,2 \\
\hline Ruanda & 10.624 & 26.003 & 1.998 & 10.192 & 18,8 & 39,2 \\
\hline Seicelle & 87 & 91 & 46 & 62 & 53,2 & 68,2 \\
\hline Somalia & 9.331 & 28.217 & 3.479 & 16.862 & 37,3 & 59,8 \\
\hline Sud Sudan & 9.948 & 22.571 & 1.777 & 7.991 & 17,9 & 35,4 \\
\hline Tanzania & 44.841 & 138.312 & 11.784 & 69.089 & 26,3 & 50,0 \\
\hline Uganda & 33.425 & 94.259 & 5.067 & 34.815 & 15,2 & 36,9 \\
\hline Africa centrale & 126.689 & 278.350 & 51.861 & 171.082 & 40,9 & 61,5 \\
\hline Angola & 19.082 & 42.334 & 11.140 & 33.004 & 58,4 & 78,0 \\
\hline Camerun & 19.599 & 38.472 & 10.096 & 27.387 & 51,5 & 71,2 \\
\hline Centrafricana Rep. & 4.350 & 8.491 & 1.688 & 4.916 & 38,8 & 57,9 \\
\hline Ciad & 11.227 & 27.252 & 2.441 & 10.295 & 21,7 & 37,8 \\
\hline Congo & 4.043 & 8.801 & 2.556 & 6.828 & 63,2 & 77,6 \\
\hline Congo Rep. Dem. & 65.966 & 148.523 & 22.248 & 85.100 & 33,7 & 57,3 \\
\hline Gabon & 1.505 & 2.784 & 1.292 & 2.582 & 85,8 & 92,7 \\
\hline Guinea equatoriale & 700 & 1.493 & 276 & 796 & 39,3 & 53,3 \\
\hline São Tomé e Príncipe & 165 & 299 & 103 & 234 & 62,0 & 78,2 \\
\hline Africa australe & 57.780 & 67.327 & 33.778 & 49.810 & 58,5 & 74,0 \\
\hline Botswana & 2.007 & 2.503 & 1.224 & 1.964 & 61,0 & 78,5 \\
\hline Lesotho & 2.171 & 2.788 & 583 & 1.517 & 26,8 & 54,4 \\
\hline Mozambico & 23.391 & 50.192 & 7.241 & 25.335 & 31,0 & 50,5 \\
\hline Namibia & 2.283 & 3.599 & 863 & 2.214 & 37,8 & 61,5 \\
\hline Sudafrica & 50.133 & 56.757 & 30.855 & 43.616 & 61,5 & 76,8 \\
\hline Swaziland & 1.186 & 1.679 & 253 & 499 & 21,3 & 29,7 \\
\hline Zambia & 13.089 & 45.037 & 5.069 & 26.816 & 38,7 & 59,5 \\
\hline Zimbabwe & 12.571 & 20.614 & 4.793 & 12.490 & 38,1 & 60,6 \\
\hline Totale & 1.022 .234 & 2.191 .599 & 400.652 & 1.264 .629 & 43,6 & 62,2 \\
\hline
\end{tabular}

Fonti: United Nations, 2013; Programme des Nations Unies pour les Etablissements Humains, Nairobi, 2014 
ti. Già nel 2030 la popolazione urbana dell'Africa (745 milioni) oltrepasserà la popolazione complessiva dell'Europa (685 milioni); inoltre, la speranza di vita alla nascita aumenterà notevolmente, mentre si abbasseranno i tassi di fertilità e i tassi di mortalità ${ }^{2}$. Del resto, sono destinate a migliorare anche l'odierna sottoalimentazione cronica e la perdurante malnutrizione (FAO, FIDA e PAM, 2013).

Nel continente oggi la maggiore incidenza della popolazione urbana su quella totale si verifica in Africa settentrionale $(51,2 \%)$, con punte elevatissime in Libia $(77,6 \%)$ e Algeria (72,0\%) ma importanti anche in Tunisia $(66,1 \%)$, tutti Stati con un'ampia componente di territorio desertico. Nondimeno, sulla sponda sud del Mediterraneo l'agglomerato con il maggior numero di abitanti è Il Cairo, un'autentica megalopoli che, con oltre $11 \mathrm{mi}$ lioni di residenti in città (diventeranno poco meno di 15 nel 2025), ospita quasi un terzo della popolazione urbana dell'intero Egitto e circa un settimo di quella nazionale ${ }^{3}$. Nell'Africa settentrionale fa la sua parte anche Alessandria, nella quale si concentrano 4.400 .000 abitanti. Sempre in questo areale, la capitale del Sudan, Khartoum, è la seconda città per peso demografico con 4 milioni e mezzo di persone, mentre in Marocco spicca Casablanca con i suoi 3 milioni.

In Africa occidentale è la Nigeria a presentare la più articolata strutturazione urbana, con un colosso come Lagos, città abitata già oggi da quasi 11 milioni di abitanti e destinata a divenire, nel 2025, la più popolata megalopoli africana con poco meno di 19 milioni di persone, una cifra pressoché pari all'attuale numero di abitanti della Costa d'Avorio. Oltre alla presenza di Lagos, l'area meridionale del Paese è caratterizzata da un addensamento di città medio-grandi, come Ibadan (2.855.000 abitanti), Ogbomosho, Ilorin, Benin-City, Onitsha, Enugu, Aba e Port-Harcourt (Tab. 2). Al centro "geometrico" della Nigeria è invece localizzata la capitale, Abuja, che per carico demografico è la quarta del Paese (2.010.000), affiancata, nell'altopiano centrale, da Kaduna e Jos. Nella parte più asciutta e settentrionale del Paese, all'estremità orientale un nucleo importante

2. Tra i primi 50 Paesi al mondo con più elevato tasso di mortalità dei bambini sotto $i$ cinque anni, 42 sono africani (UNICEF, 2012, pp. 87 ss.). Correlativamente, i Paesi africani occupano le ultime posizioni nella graduatoria mondiale della speranza di vita alla nascita, indicatore nel quale, mediamente, le persone raggiungono a malapena i cinquant'anni d'età, contro gli oltre ottant'anni dei cittadini di Italia e Giappone al vertice opposto (UNDP, 2012, pp. 154 ss.).

3. Non solo: gli abitanti del Cairo superano la popolazione totale della Tunisia e sono pressoché pari a tutta la popolazione urbana del Sudan. Ma, per meglio definire comparativamente il peso demografico di questa città, si pensi che alla scala continentale essa ha un numero di abitanti paragonabile (talvolta di poco superiore, talora lievemente inferiore) a quello di Paesi come il Benin, la Guinea (Conakry), il Senegal, il Ruanda, la Somalia, il Sud Sudan, la Repubblica Centrafricana o lo Zimbabwe. 
Tab. 2 - Abitanti delle maggiori agglomerazioni urbane in Africa (in migliaia)

\begin{tabular}{|c|c|c|c|c|c|}
\hline Nazioni & Città & 1960 & 1980 & 2010 & $2025^{*}$ \\
\hline \multicolumn{6}{|l|}{ Africa settentrionale } \\
\hline Algeria & Algeri & 872 & 1.621 & 2.851 & 3.977 \\
\hline Egitto & Alessandria & 1.504 & 2.519 & 4.400 & 6.189 \\
\hline Egitto & II Cairo & 3.680 & 7.349 & 11.031 & 14.740 \\
\hline Libia & Tripoli & 174 & 662 & 1.111 & 1.456 \\
\hline Marocco & Casablanca & 967 & 2.109 & 3.009 & 3.911 \\
\hline Marocco & Fès & 280 & 510 & 1.065 & 1.455 \\
\hline Marocco & Rabat & 233 & 808 & 1.807 & 2.429 \\
\hline Sudan & Khartoum & 347 & 1.164 & 4.516 & 7.090 \\
\hline \multicolumn{6}{|l|}{ Africa occidentale } \\
\hline Burkina Faso & Ouagadougou & 59 & 265 & 1.911 & 4.795 \\
\hline Costa d'Avorio & Abidjan & 192 & 1.384 & 4.151 & 6.971 \\
\hline Ghana & Accra & 393 & 863 & 2.342 & 3.497 \\
\hline Guinea & Conakry & 112 & 658 & 1.715 & 3.195 \\
\hline Mali & Bamako & 130 & 489 & 1.932 & 3.632 \\
\hline Niger & Niamey & 58 & 274 & 1.222 & 2.924 \\
\hline Nigeria & Abuja & 23 & 125 & 2.010 & 4.000 \\
\hline Nigeria & Benin City & 83 & 335 & 1.311 & 2.377 \\
\hline Nigeria & Ibadan & 570 & 1.186 & 2.855 & 5.028 \\
\hline Nigeria & Kaduna & 99 & 628 & 1.476 & 2.633 \\
\hline Nigeria & Kano & 229 & 1.350 & 3.271 & 5.724 \\
\hline Nigeria & Lagos & 762 & 2.572 & 10.788 & 18.857 \\
\hline Nigeria & Ogbomosho & 247 & 485 & 1.039 & 1.884 \\
\hline Nigeria & Port Harcourt & 135 & 482 & 1.807 & 3.371 \\
\hline Senegal & Dakar & 349 & 957 & 2.926 & 5.064 \\
\hline Togo & Lomé & 95 & 344 & 1.453 & 2.472 \\
\hline \multicolumn{6}{|l|}{ Africa orientale } \\
\hline Etiopia & Addis Abeba & 519 & 1.175 & 2.919 & 4.705 \\
\hline Kenya & Nairobi & 290 & 862 & 3.237 & 6.143 \\
\hline Madagascar & Antananarivo & 252 & 580 & 1.900 & 3.898 \\
\hline Somalia & Mogadiscio & 94 & 551 & 1.426 & 3.309 \\
\hline Tanzania & Dar es Salaam & 162 & 836 & 3.415 & 7.276 \\
\hline Uganda & Kampala & 137 & 469 & 1.594 & 3.540 \\
\hline \multicolumn{6}{|l|}{ Africa centrale } \\
\hline Angola & Huambo & 37 & 153 & 1.039 & 1.997 \\
\hline Angola & Luanda & 219 & 962 & 4.790 & 8.924 \\
\hline Camerun & Douala & 153 & 571 & 2.348 & 3.983 \\
\hline Camerun & Yaoundé & 75 & 415 & 2.320 & 3.997 \\
\hline Ciad & N'Djamena & 70 & 324 & 1.038 & 1.870 \\
\hline Congo & Brazzaville & 124 & 446 & 1.557 & 2.364 \\
\hline Congo Rep. Dem. & Kinshasa & 443 & 2.053 & 8.415 & 14.535 \\
\hline Congo Rep. Dem. & Lubumbashi & 194 & 482 & 1.486 & 2.689 \\
\hline Congo Rep. Dem. & Mbuji-Mayi & 136 & 407 & 1.433 & 2.606 \\
\hline \multicolumn{6}{|l|}{ Africa australe } \\
\hline Mozambico & Maputo & 181 & 550 & 1.132 & 1.823 \\
\hline Sudafrica & Città del Capo & 803 & 1.609 & 3.492 & 4.388 \\
\hline Sudafrica & Durban & 677 & 1.214 & 2.954 & 3.724 \\
\hline Sudafrica & East Rand & 682 & 1.107 & 3.284 & 4.150 \\
\hline Sudafrica & Johannesburg & 1.147 & 1.656 & 3.763 & 4.732 \\
\hline Sudafrica & Port Elizabeth & 289 & 590 & 1.097 & 1.418 \\
\hline Sudafrica & Pretoria & 419 & 688 & 1.468 & 1.894 \\
\hline Sudafrica & Vereeniging & 187 & 451 & 1.174 & 1.522 \\
\hline Zambia & Lusaka & 91 & 533 & 1.719 & 3.496 \\
\hline Zimbabwe & Harare & 248 & 616 & 1.526 & 2.337 \\
\hline
\end{tabular}

(* proiezioni)

Fonti: Farvacque-Vitkovic, Raghunath, Eghoff e Boakye, 2008, p. 2; United Nations Human Settlements Programme, 2010, p. 244; Programme des Nations Unies pour les Etablissements Humains, 2014 
di popolazione si concentra a Maiduguri, ma a farla da padrone nel nord è Kano, seconda città della Nigeria per numero di abitanti (3.271.000). Per fornire un'immagine della situazione, si pensi che nel 2025 questi 14 agglomerati urbani accoglieranno complessivamente quasi 53 milioni di persone, una popolazione di poco inferiore a quelle attuali di Spagna e Portogallo prese assieme.

Tra le altre città di grande peso demografico in Africa occidentale troviamo Abidjan, capitale economica della Costa d'Avorio (4.151.000 abitanti), destinata a raggiungere i 7 milioni di residenti entro il primo quarto del secolo. Ad essa si aggiungono sia Dakar (2.926.000), capitale politica e culturale del Senegal, che nel 2025 ne avrà 5 milioni, sia Accra (2.342.000), capitale del Ghana e agglomerato urbano destinato ad aumentare di 100.000 abitanti all'anno nel prossimo decennio (Farvacque-Vitkovic, Raghunath, Eghoff e Boakye, 2008, p. 2). Vengono poi diverse capitali ovest-africane come Bamako in Mali, Ouagadougou in Burkina Faso, Conakry in Guinea, Lomé in Togo, Niamey in Niger, tutte votate a una crescita popolazionistica importante, se si pensa che Ouagadougou si approssimerà ai 5 milioni di persone nell'orizzonte del 2025, Bamako supererà i 3 milioni e mezzo, Conakry oltrepasserà i 3 milioni e Niamey li sfiorerà, mentre Lomé si avvicinerà ai 2 milioni e mezzo. Insomma, l'Africa occidentale può essere identificata come la macroregione del continente in cui potenzialità e guasti dello sviluppo urbano faranno le loro prove maggiori e potranno alimentare quei push factor che influiscono sui flussi migratori.

In Africa orientale, attualmente l'ambito regionale più popoloso del continente, sulla costa dell'oceano Indiano spicca Dar es Salaam, capitale della Tanzania, con quasi 3 milioni e mezzo di abitanti destinati a duplicarsi nei prossimi dieci anni. Sull'altopiano kenyota di blixeniana memoria emerge poi, per consistenza della popolazione urbana, la capitale Nairobi (3.237.000 persone, supererà i 6 milioni nel 2025), mentre nel Corno d'Africa, sull'altopiano etiopico a tremila metri d'altitudine, sfiora i 3 milioni d'individui Addis Abeba (4.705.000 fra un decennio), ribattezzata "eucaliptopoli", capitale di questo grande Paese che ha subito il colonialismo italiano. Al confine sud-orientale dell'Etiopia, in terra somala si distingue ancora Mogadiscio, nonostante sia stata martoriata negli ultimi decenni dalle lotte intestine dei warlord. A loro volta Kampala, capitale dell'Uganda localizzata poco lontano dalle rive del lago Vittoria, e Antananarivo, centro di governo politico della grande isola del Madagascar, sono votate, insieme alla capitale somala, a più che raddoppiare la loro popolazione urbana entro il primo quarto del secolo (UN-Habitat, 2010, pp. 238 ss.).

Dopo Il Cairo e Lagos, è Kinshasa la terza megalopoli del continente, con otto milioni e mezzo di abitanti, largamente sottostimati, che saranno 
circa quindici fra un decennio. Ciò significa che ai giorni nostri, nella Repubblica Democratica del Congo, un abitante su otto vive nella capitale (e probabilmente l'incidenza è assai più marcata di quanto non attestino i dati ufficiali) ${ }^{4}$. In Africa centrale, un'altra città destinata a diventare una bomba demografica è Luanda, oggi abitata da 5 milioni di persone, mentre nel 2025 ne registrerà nove, senza contare che nel 2050 in Angola 8 persone su 10 abiteranno in ambiente urbano. Completano il quadro di questo areale, tra gli agglomerati urbani ancora significativi per carico demografico, Douala in Camerun, Brazzaville in Congo e N'Djamena in Ciad.

Nella regione australe giganteggia, invece, Johannesburg con quasi quattro milioni di abitanti; se, però, nella sua vastissima area metropolitana venissero inclusi l'East Rand (Ekurhuleni) e il West Rand, l'aggregato urbano e la sua periferia contrassegnata da bidonvilles conterebbero una popolazione di circa 10 milioni di persone. Ma il Sudafrica gioca un ruolo ben più significativo, paragonabile a quello svolto dalla Nigeria in Africa occidentale sotto il profilo della strutturazione urbana: infatti, aggiungendo anche solo Città del Capo, Durban, Pretoria, Vereeniging e Port Elizabeth alla grande Johannesburg, nel 2025 queste città aggregherebbero 26 milioni di individui, cioè più della metà dell'attuale popolazione sudafricana complessiva. Nella parte meridionale del continente un posto rilevante occupano infine Harare e Maputo, ma anche Lusaka, la sola di queste città in cui si prevede un raddoppio della popolazione nel prossimo decennio.

Insomma, questo continente così vicino alle nostre coste, che ha ormai raggiunto e superato il miliardo di abitanti, preoccupa l'opinione pubblica ma crea un certo disagio anche tra gli studiosi. E ancora oggi il disagio, com'era stato lucidamente sostenuto già vent'anni or sono, resta basato soprattutto sulla «consapevolezza che l'aumento imperioso della circolazione di uomini e il passaggio attraverso le frontiere più "molli" (Italia e Spagna) del Sud comunitario rendano assai problematico lo sbarrare le porte, che l'enorme pressione derivante dalla transizione demografica del Mediterraneo tenda quasi senza rimedio a superare ogni cancello esportandoci ampie fette di Terzo Mondo in casa» (Coppola, 1994, p. 11). In certa misura ciò è poi avvenuto, ma, come avremo modo di vedere, non ha prodotto quegli effetti catastrofici che una parte importante della società italiana, insofferente verso la presenza dei migranti sul territorio della penisola, perciò critica riguardo a ogni forma di multiculturalismo o intercultura, aveva paventato e continua oggi a temere.

4. Peraltro, una consistente comunità di congolesi (circa 5.000 migranti, che nel 2007 superavano appena le 3.000 unità) è già oggi presente nel nostro Paese (D’Ascenzo, 2010, pp. 139 ss.). 


\subsection{L'Italia tra Europa e Africa: una geografia dei flussi}

I fenomeni migratori hanno in Africa una rilevanza forse più marcata che in ogni altro continente - e non solo perché le rimesse dei migranti, cioè gli invii di denaro ai loro Paesi d'origine, hanno ormai raggiunto un volume finanziario tre volte superiore a quello dell'aiuto pubblico allo sviluppo, determinando un impatto non trascurabile sulla povertà e sulla sicurezza alimentare. Le migrazioni, infatti, sono importanti anche perché chi ne è protagonista può essere coinvolto nella crescita dei sistemi socioeconomici di provenienza, sia promuovendo dal luogo di approdo scambi commerciali con essi, sia rientrando direttamente per mettere a disposizione le proprie competenze acquisite. Del resto, in presenza di adeguate politiche nazionali o locali, i migranti possono generare un aumento degli investimenti in settori differenti, ivi compreso quello dello sfruttamento agricolo. Tuttavia, per restare alle rimesse, l'apporto monetario della diaspora africana è veramente imponente: nel continente arrivano annualmente circa 40 miliardi di dollari, quattro dei quali giungono da altri Paesi africani, in particolare dai migranti che vivono in Sudafrica - peraltro, primo Paese al mondo per accoglienza di richiedenti asilo (Nanni e Pittau, 2010). Le rimesse hanno una consistente incidenza sul Pil, specialmente in alcuni Paesi (28\% nel Lesotho, 10\% nel Senegal e nel Togo, 9\% a Capo Verde). La Nigeria è invece il principale ricettore di rimesse $(9,5$ miliardi di dollari nel 2008), seguita da Egitto (7,8 miliardi) e Marocco (5,7 miliardi). In anni recenti l'invio di rimesse da parte degli immigrati africani in Italia si è attestato sul miliardo di euro, di cui circa la metà verso il Nord Africa.

In ogni modo conviene sottolineare che le migrazioni, forzate o volontarie, sono soprattutto interne al continente (Wihtol de Wenden, 2009, p. 45). Si fugge da un Paese implicato in una "guerra a bassa intensità" per recarsi in un altro capace di offrire rifugio: basti pensare alle situazioni della Somalia, dell'Eritrea, della Repubblica Democratica del Congo o ad altri contesti di conflitto. Secondo l'UNHCR, alla fine del 2013 in Africa gli sfollati interni erano oltre 7 milioni e mezzo, cioè un terzo di tutti quelli esistenti al mondo, mentre i rifugiati e i richiedenti asilo hanno quasi raggiunto i 3 milioni e mezzo. Risultano intense anche le migrazioni più propriamente economiche. $\mathrm{Ci}$ si sposta dalle aree agricole a quelle urbane, ma ci si dirige anche da un Paese meno prospero verso altri che offrono maggiori prospettive occupazionali, come il Sudafrica o i Paesi del Nord Africa. I migranti che dalla fascia subsahariana si trasferiscono ogni anno nel Maghreb sono tra i 65.000 e i 120.000: non sempre si tratta di un insediamento definitivo, perché l'intenzione è solitamente di passare in Europa e una quota importante, stimata tra il $20 \%$ e il $38 \%$, tenta la traversata del Medi- 
terraneo. La migrazione interna è spesso solo una tappa intermedia di un viaggio diretto oltre i confini del continente.

Le partenze verso l'Europa, se irregolari, prendono solitamente la via del mare e si dirigono verso le Canarie (ma anche a Ceuta e Melilla) e, al di fuori del continente, le Baleari, l'Andalusia, Malta, la Sicilia (in particolare le isole di Lampedusa e Pantelleria), la Sardegna (almeno dal 2006 con partenze dall'Algeria) e la Grecia (transitando attraverso la Turchia). Tutti questi spostamenti, com'è ormai da tempo tristemente noto, non di rado sono contrassegnati da naufragi o altre circostanze mortali ${ }^{5}$. Più in generale, con il coinvolgimento di molte altre nazionalità non solo africane (in specie provenienti dall'Asia mediorientale), dal 2006 al 2013 il meridione d'Europa ha fatto registrare un andamento annuale fluttuante degli sbarchi in Grecia (tra un minimo di circa 1.000 e un massimo di poco inferiore ai 20.000) e a Malta (da un massimo di 2.700 nel 2008 a un minimo di 28 nel 2010), mentre è stato progressivamente decrescente in Spagna (39.180 sbarchi nel 2006 contro 3.235 nel 2013) e crescente in Italia, con un picco nel 2011 (62.692, in concomitanza delle "primavere arabe" nel Maghreb, particolarmente in Egitto, Libia e Tunisia), seguito al valore più basso in assoluto dell'anno precedente (4.406). In otto anni è stata l'Italia ad accogliere complessivamente il maggior numero di migranti irregolari (214.285), lasciando distanziate Spagna (93.979), Grecia (72.267) e Malta (13.270). Nei primi mesi del 2014, fino ad agosto, i flussi verso la penisola sono poi aumentati vertiginosamente, raggiungendo una cifra pari a circa la metà di tutti gli sbarchi verificatisi negli otto anni precedenti $(112.689)^{6}$. In tre lustri, dal 1999 al 2014, i dati disponibili e accertati mostrano che sono giunti in Italia con i "barconi" poco meno di mezzo milione di migranti.

Agli inizi del 2013, dei circa cinque milioni di africani regolarmente presenti nell'Unione Europea, oltre un quinto risultava insediato in Italia (Tab. 3). Qui appaiono nettamente prevalenti le nazionalità nordafricane, tanto che nel 2009 in sette casi su dieci i migranti erano originari dei Paesi affacciati sul Mediterraneo e, in cinque casi su dieci, provenivano dal Marocco. Gli immigrati africani, in quello stesso anno, vivevano nel settentrione della penisola in tre casi su quattro e per i due terzi in sole quattro regioni: Lombardia, Emilia-Romagna, Veneto e Piemonte. Nel 2013, all'inizio dell'anno i migranti regolari, in prevalenza maschi, avevano superato di gran lunga il milione di presenze, di cui più di un quinto composto da minori. I lavoratori d'origine africana costituivano una quota importante

5. Su cui, fin dagli inizi del terzo Millennio, aveva focalizzato un'attenzione critica Delle Donne (2004).

6. Di cui 81.973 in Sicilia (3.741 nella sola Lampedusa), 12.634 in Puglia, 11.254 in Calabria, 111 in Sardegna e 6.717 in altre regioni (de Donato e Stifano, 2014, p. 55). 
Tab. 3 - Migranti africani in Italia per nazionalità e sesso (2008-2012)

\begin{tabular}{|c|c|c|c|c|c|c|c|}
\hline Nazionalità & $\begin{array}{c}\text { Presenze } \\
31.12 .2008\end{array}$ & $M$ & $F$ & Nazionalità & $\begin{array}{c}\text { Presenze } \\
31.12 .2012 \\
\end{array}$ & $M$ & $F$ \\
\hline Marocco & 406.341 & 236.021 & 170.320 & Marocco & 513.374 & 288.242 & 225.132 \\
\hline Tunisia & 102.375 & 66.150 & 36.225 & Egitto & 123.529 & 87.592 & 35.937 \\
\hline Egitto & 77.750 & 54.408 & 23.342 & Tunisia & 121.483 & 77.525 & 43.958 \\
\hline Senegal & 68.194 & 53.807 & 14.387 & Senegal & 92.371 & 68.401 & 23.970 \\
\hline Nigeria & 45.743 & 20.365 & 25.378 & Nigeria & 66.631 & 32.675 & 33.956 \\
\hline Ghana & 42.937 & 24.262 & 18.675 & Ghana & 56.021 & 33.452 & 22.569 \\
\hline Algeria & 24.951 & 16.951 & 8.000 & Algeria & 28.617 & 18.254 & 10.363 \\
\hline Costa d'Avorio & 19.589 & 10.871 & 8.718 & Costa d'Avorio & 26.599 & 15.343 & 11.256 \\
\hline Eritrea & 11.949 & 6.405 & 5.544 & Burkina Faso & 15.662 & 10.287 & 5.375 \\
\hline Maurizio & 10.532 & 6.829 & 3.703 & Camerun & 12.602 & 6.576 & 6.026 \\
\hline Burkina Faso & 9.999 & 4.706 & 5.293 & Eritrea & 11.493 & 5.830 & 5.663 \\
\hline Etiopia & 9.330 & 3.794 & 5.536 & Etiopia & 9.420 & 3.535 & 5.885 \\
\hline Camerun & 8.178 & 3.972 & 4.206 & Maurizio & 8.934 & 4.004 & 4.930 \\
\hline Somalia & 8.165 & 4.325 & 3.840 & Somalia & 8.668 & 5.591 & 3.077 \\
\hline Capo Verde & 5.215 & 1.484 & 3.731 & Togo & 5.380 & 3.456 & 1.924 \\
\hline Congo & 3.987 & 2.077 & 1.910 & Capo Verde & 5.200 & 1.564 & 3.636 \\
\hline Togo & 3.854 & 2.547 & 1.307 & Guinea (Conakry) & 4.687 & 3.106 & 1.581 \\
\hline Rep. Dem. Congo & 3.667 & 1.908 & 1.759 & Rep. Dem. Congo & 4.321 & 2.224 & 2.097 \\
\hline Guinea (Conakry) & 2.749 & 1.751 & 998 & Mali & 4.236 & 3.697 & 539 \\
\hline Benin & 2.530 & 2.154 & 376 & Congo & 4.034 & 2.076 & 1.958 \\
\hline Sudan & 2.317 & 1.367 & 950 & Benin & 2.933 & 1.724 & 1.209 \\
\hline Liberia & 2.141 & 1.830 & 311 & Sudan & 2.862 & 2.407 & 455 \\
\hline Angola & 1.877 & 1.224 & 653 & Kenya & 2.295 & 907 & 1.388 \\
\hline Libia & 1.745 & 933 & 812 & Liberia & 1.814 & 1.514 & 300 \\
\hline Kenya & 1.445 & 607 & 838 & Angola & 1.700 & 890 & 810 \\
\hline Sierra Leone & 1.278 & 779 & 499 & Libia & 1.648 & 1.104 & 544 \\
\hline Madagascar & 1.169 & 309 & 860 & Gambia & 1.559 & 1.244 & 315 \\
\hline Niger & 1.121 & 510 & 611 & Madagascar & 1.401 & 412 & 989 \\
\hline Mali & 1.034 & 617 & 417 & Sierra Leone & 1.389 & 811 & 578 \\
\hline Tanzania & 924 & 404 & 520 & Niger & 1.352 & 945 & 407 \\
\hline Gambia & 833 & 384 & 449 & Tanzania & 1.073 & 498 & 575 \\
\hline Seicelle & 825 & 591 & 234 & Mauritania & 1.010 & 708 & 302 \\
\hline Sudafrica & 650 & 310 & 340 & Sudafrica & 718 & 287 & 431 \\
\hline Mauritania & 572 & 305 & 267 & Ciad & 688 & 568 & 120 \\
\hline Rwanda & 571 & 260 & 311 & Rwanda & 610 & 303 & 307 \\
\hline Burundi & 568 & 388 & 180 & Uganda & 599 & 295 & 304 \\
\hline Uganda & 497 & 253 & 244 & Seicelle & 594 & 166 & 428 \\
\hline Guinea-Bissau & 347 & 202 & 145 & Burundi & 588 & 243 & 345 \\
\hline Mozambico & 286 & 120 & 166 & Guinea-Bissau & 562 & 394 & 168 \\
\hline Zambia & 262 & 158 & 104 & Mozambico & 401 & 164 & 237 \\
\hline Gabon & 228 & 114 & 114 & Zambia & 274 & 140 & 134 \\
\hline Ciad & 156 & 96 & 60 & Gabon & 203 & 99 & 104 \\
\hline Rep. Centrafricana & 142 & 86 & 56 & Zimbabwe & 179 & 78 & 101 \\
\hline Guinea Equatoriale & 128 & 59 & 69 & Rep. Centrafricana & 138 & 79 & 59 \\
\hline Zimbabwe & 98 & 54 & 44 & Guinea Equatoriale & 107 & 57 & 50 \\
\hline Malawi & 48 & 23 & 25 & Malawi & 64 & 32 & 32 \\
\hline Gibuti & 41 & 27 & 14 & São Tomé e Príncipe & 30 & 13 & 17 \\
\hline São Tomé e Príncipe & 35 & 14 & 21 & Botswana & 26 & 15 & 11 \\
\hline Namibia & 16 & 4 & 12 & Gibuti & 26 & 15 & 11 \\
\hline Lesotho & 14 & 7 & 7 & Namibia & 25 & 7 & 18 \\
\hline Swaziland & 14 & 8 & 6 & Lesotho & 23 & 13 & 10 \\
\hline Botswana & 12 & 9 & 3 & Swaziland & 21 & 14 & 7 \\
\hline Comore & 6 & 6 & 0 & Comore & 3 & 3 & 0 \\
\hline TOTALE & 889.435 & 536.845 & 352.590 & TOTALE & 1.150 .177 & 689.579 & 460.598 \\
\hline
\end{tabular}

Fonti: Nostre elaborazioni su dati pluriennali Istat e Ministero dell'Interno 
di tutti gli occupati nati all'estero (poco meno del 20\%), con un'accentuata concentrazione nel settore industriale.

Oltre alle nazionalità predominanti del Nord Africa, tra i migranti provenienti da Paesi a sud del Sahara emergevano i cittadini del Senegal, della Nigeria, del Ghana e della Costa d'Avorio che, nell'insieme, costituivano i due terzi di tutti gli immigrati subsahariani presenti in Italia nel 2013. E i mutamenti più corposi nelle presenze, verificatisi dal 2008 al 2012, hanno riguardato soprattutto migranti partiti da questo vasto areale: i maliani si sono praticamente quadruplicati, i gambiani sono aumentati dell' $87,2 \%$, i mauritani del $76,6 \%$, i guineani del $70,5 \%$, i burkinabé del $56,6 \%$ e i camerunesi del $54,1 \%$. Nel complesso la crescita della popolazione migrante nel quadriennio ha raggiunto il 29,3\%. Questi flussi migratori, che oggi destano preoccupazione in Italia ma anche in Europa soprattutto quando li si collega erroneamente solo agli sbarchi irregolari in Sicilia e a Lampedusa, nel prossimo futuro saranno probabilmente artefici di un mutamento e di un riequilibrio popolazionistico tra Paesi poveri e Paesi ricchi, vista la stasi demografica nel vecchio continente e la crescita vistosa nel numero degli abitanti in Africa. E mentre sono già una fonte importante di introiti per lo Stato, si può immaginare un domani in cui il ruolo economico dei migranti e dei loro figli, vale dire le seconde e terze generazioni, tenderà a crescere incidendo sul tessuto sociale di molti Paesi europei.

In questo contesto, dunque, le migrazioni internazionali non potranno non avere un ruolo importante anche negli anni a venire. Ma, intanto, già oggi hanno un impatto positivo sullo sviluppo dei Paesi in difficoltà, incrementabile a condizione che in un tempo auspicabilmente prossimo vengano attuate politiche di sostegno adeguate e sia garantita la tutela dei diritti umani, tanto nei Paesi d'origine quanto in quelli di transito e destinazione. Si pensi che, in sei anni di crisi economica globale, le rimesse dei $232 \mathrm{mi}-$ lioni di migranti nel mondo, pari a circa 550 miliardi di euro all'anno, hanno fatto registrare un moderato ma costante aumento (Luatti, 2014, pp. 26 ss.). La tendenziale anticiclicità di questa fonte finanziaria porta certo benefici nei Paesi di esodo dei migranti (dove è una risorsa assai superiore agli aiuti allo sviluppo), così come l'alto numero d'imprese e attività economiche da essi avviate in quelli di approdo genera effetti importanti per i territori nazionali che li accolgono. Senza contare il contributo ineludibile che essi offrono in settori di lavoro spesso "disertati" dai cittadini autoctoni.

Nei 27 Paesi dell'Unione Europea vivono oltre 34 milioni di migranti, circa il 7\% sul totale dei residenti, con Germania (7,4 milioni), Spagna (5,5), Italia $(5,0)$, Regno Unito $(4,8)$ e Francia $(3,8)$ che, nell'insieme, ospitano quattro quinti degli stranieri presenti nell'UE. Nel 2011 dall'Europa sono stati inviati complessivamente 40 miliardi di rimesse ai Paesi d'origine degli immigrati e il maggior volume finanziario in uscita proviene proprio dalle Nazioni in cui 
si trovano le popolazioni straniere numericamente più consistenti. Insomma, i migranti mettono in circolazione denaro, ma non solo: importano prodotti dai loro Paesi e lì esportano quelli dei luoghi in cui si sono insediati, alimentano il transnazionalismo del web e delle telecomunicazioni, dei viaggi e dei rientri per ricorrenze festive o per pause di lavoro, fanno girare idee e tradizioni diverse. Sono il segno evidente del fatto che la mobilità geografica dei gruppi umani è diventata una componente imprescindibile della globalizzazione. Anche e soprattutto per il futuro, dunque, i migranti costituiscono una potenzialità economica e culturale che sarebbe imperdonabile sprecare.

\subsection{Gli immigrati nel Belpaese ai tempi della crisi}

Agli inizi del 2014 gli stranieri residenti in Italia sono risultati 4.922 .085 su una popolazione complessiva di 60.782.668, con un'incidenza dell' $8,1 \%$. Il loro livello d'istruzione è notevole: il $10,3 \%$ ha una laurea e il $32,4 \%$ un diploma (dati del Censimento 2011). Le donne sono il 52,7\% e i minori oltre un milione, di cui 802.785 iscritti a scuola nell'a.s. 2013/14 (con un'incidenza del 9,0\% sul totale degli iscritti). Secondo le stime del Centro Studi e Ricerche IDOS, la presenza effettiva dei migranti in posizione regolare è però più alta e ammonta a 5.364 .000 persone (IDOS, 2014, pp. 113-14). Rispetto al periodo antecedente la crisi, le quote d'ingresso di nuovi lavoratori sono molto diminuite. Attualmente hanno maggiore peso sull'aumento della popolazione straniera i visti per ricongiungimento familiare e le nuove nascite. Notevole è anche l'incidenza delle famiglie con almeno uno straniero, che in un quarto dei casi sono miste.

Al Censimento del 1981 vi era un cittadino straniero ogni 250 residenti (incidenza dello $0,4 \%$ ), mentre ora la quota sulla popolazione totale ha raggiunto l' $8 \%$ (1 ogni 12 residenti) e in 27 province supera il 10\%, con punte massime in alcuni piccoli comuni, nei quali l'incidenza raggiunge anche il 30\%. Nonostante il policentrismo delle provenienze (196 Paesi), si riscontra una notevole prevalenza di alcune aree d'origine: circa la metà $(51,1 \%)$ proviene da soli cinque Paesi (Romania, Albania, Marocco, Cina e Ucraina), mentre diventano quasi due terzi (64\%) se si tiene conto delle prime dieci nazionalità (Tab. 4).

Un quarto della popolazione straniera risiede in quattro province (Roma, Milano, Torino e Brescia). I residenti in Lombardia (oltre un milione) rappresentano il $22,9 \%$ del totale nazionale e quelli della provincia di Roma (oltre mezzo milione) il 10,3\%. La Lombardia, l'Emilia-Romagna e, in parte, il Lazio sono le regioni in cui le appena richiamate collettività realizzano le presenze più consistenti, salvo il caso dei cinesi (per il 17\% insediati in Toscana) e degli ucraini (per il $18,5 \%$ in Campania). La crisi continua a 


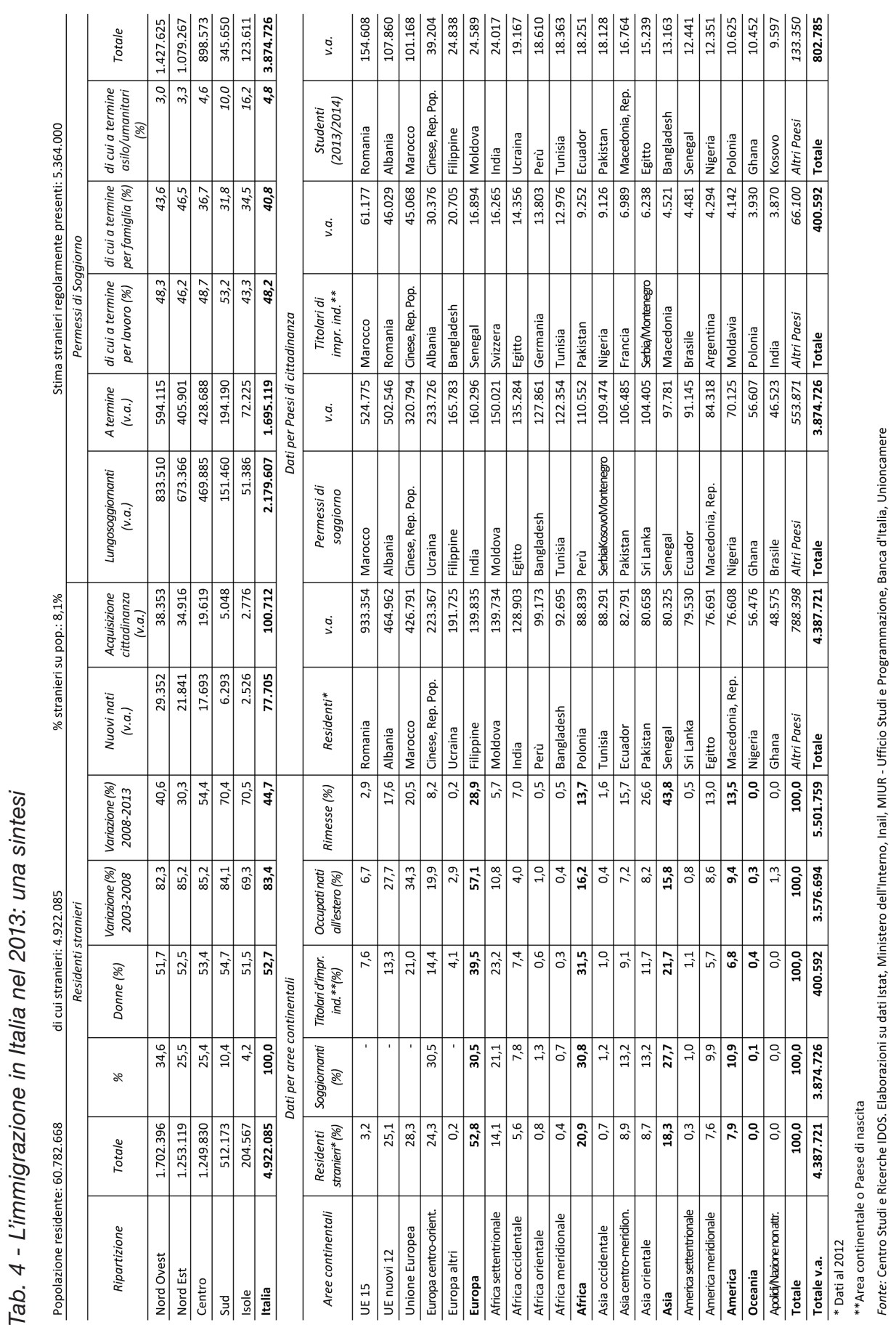


far sentire i suoi effetti negativi anche sul fenomeno migratorio. I permessi scaduti senza essere rinnovati sono stati 262.688 nel 2011, 166.321 nel 2012 e 145.670 nel 2013. Tuttavia, ufficialmente, le partenze per l'estero hanno coinvolto 44.000 cittadini stranieri.

Stando ai dati del Censimento 2011, in media la differenza d'età tra stranieri e italiani è di 13 anni (31,1 rispetto a 44,2) e questo divario fa sì che l'immigrazione influisca positivamente sul sistema pensionistico. Secondo stime recenti la quota attuale di immigrati che ha raggiunto l'età pensionabile si attesta al 2,6\% sul totale dei casi, ma essa salirà al 4,3\% nel 2020 e al 6,0\% nel 2025. Anche i cittadini italiani per acquisizione, che erano solo 285.785 nel 2001, sono aumenti a 671.394 , cui se ne sono aggiunti 65.383 che hanno ottenuto la cittadinanza nel 2012 e 100.712 nel 2013. In un terzo dei casi il riconoscimento della cittadinanza è avvenuto per matrimonio.

Come abbiamo già visto, sono in aumento pure gli sbarchi dei profughi provenienti dall'Africa e dall'Asia mediorientale, con diverse migliaia di migranti morti nel Mediterraneo nonostante l'operazione "Mare Nostrum" iniziata il 18 ottobre 2013, operazione che in un anno ha permesso di salvare oltre 130.000 persone, malgrado la mancanza di un accordo a livello comunitario per la ripartizione tra i Paesi europei degli oneri relativi a pattugliamento, salvataggio e accoglienza. Nel 2013 il numero delle richieste d'asilo in Italia è risultato contenuto (26.620) rispetto ad altri Paesi europei (la Germania, per esempio, ne ha ricevute 127.000). Il 10 luglio 2014 è intervenuto un accordo tra Stato, Regioni ed Enti Locali per un piano nazionale di accoglienza che, nel triennio 2014-2016, aumenterà da 3.000 a quasi 20.000 i posti messi a disposizione dal Sistema di accoglienza per richiedenti asilo e rifugiati.

Risultano in diminuzione le persone non autorizzate all'ingresso intercettate alle frontiere italiane (7.713), le persone rimpatriate (8.769) e quelle cui è stata intimata l'espulsione ma non hanno ottemperato (13.529) - per un totale di 30.011 individui, in costante diminuzione dal 2006 (quando furono 124.381). Secondo una convinzione prevalente, la popolazione straniera in posizione irregolare dovrebbe essere diminuita a meno di mezzo milione, anche perché 430.000 non autorizzati a stare in Italia sono stati interessati dai provvedimenti di emersione varati nel 2009 e nel 2012. Una realtà molto problematica è quella dei Centri di identificazione ed espulsione. $\mathrm{Su} 420$ Cie operanti in tutta l'UE (37.000 posti in totale), 10 si trovano in Italia, dove nel 2013 sono stati trattenuti 5.431 uomini e 585 donne, con una permanenza media di 38 giorni, un tasso di espulsioni eseguite pari al $45,7 \%$ e condizioni di vita critiche per quel che riguarda il rispetto dei diritti umani. In queste strutture di detenzione amministrativa, dove la possibilità di trattenimento è salita a 18 mesi a partire dal 2011, sono continue le proteste e le rivolte. 
Quanto poi al mondo del lavoro, l'incidenza degli stranieri sul totale degli occupati era del 3,2\% nel 2001 ed è aumentata nel 2011 all' $8,2 \%$. I dati dell'ISTAT attestano che nel 2013 si è trattato di 2,5 milioni di lavoratori, diminuiti a 2 milioni alla fine dello stesso anno, con un aumento di circa 1.000 disoccupati al mese nel corso dell'ultimo biennio (complessivamente 636.000). Tra i lavoratori stranieri il tasso di disoccupazione è salito nel 2013 al 17,3\%. La crisi ha influito anche sull'invio delle rimesse che, pur rimanendo essenziali per le famiglie e i Paesi d'origine degli immigrati, sono ulteriormente diminuite nel 2013 a 5,5 miliardi di euro, circa un quinto in meno rispetto all'anno precedente e con un notevole ribasso rispetto al picco raggiunto nel 2011 (7,4 miliardi di euro).

\subsection{Figli della mobilità: un territorio per le seconde gene- razioni}

Data la consistente presenza di migranti sul territorio, nel contesto italiano attuale sta crescendo il fenomeno dei matrimoni misti, uno dei più significativi processi interculturali e d'integrazione dei cittadini stranieri nella società d'accoglienza. Tali unioni, che nel 2009-2010 avevano fatto registrare una battuta d'arresto a causa del "pacchetto sicurezza" (legge Maroni n. 94 del 2009) con cui si era introdotto l'obbligo per il migrante di attestare la regolarità del soggiorno se voleva convolare a nozze, sono tornate ad aumentare in modo netto. Nel 2012 i matrimoni con almeno un coniuge straniero sono stati quasi 31.000 , cioè 5.000 in più rispetto al 2010 , pari a circa il 15\% sul totale delle celebrazioni (Gaffuri, 2014, pp. 211 ss.). Durante il medesimo anno, nelle oltre ventimila coppie miste in Italia, la tipologia più diffusa ha riguardato le unioni tra un coniuge italiano e una sposa straniera, con una disparità anagrafica dei coniugi più marcata che nel caso delle coppie dei nostri concittadini: sono state 16.340 le nozze di questo tipo, mentre 4.424 hanno visto come protagonisti una donna italiana e un partner straniero.

Quanto poi alla cittadinanza dei coniugi coinvolti, lo sposo italiano contrae matrimonio con una romena nel $17,4 \%$ dei casi, il $10,9 \%$ prende in moglie un'ucraina e il 7,2\% convola a nozze con una brasiliana. Nella caratterizzazione contraria, vale a dire quando si tratta di spose italiane, esse si maritano nel $15,0 \%$ dei casi con marocchini, per il 7,8\% celebrano unioni con albanesi e il 7,6\% di esse contrae matrimonio con tunisini. In ogni caso, si tratta sempre di coppie nelle quali i partner stranieri provengono da Paesi in cui la pressione migratoria è assai elevata. Tra le unioni miste con almeno un coniuge straniero, nel 2012 la stragrande maggioranza del- 
le celebrazioni è stata di tipo civile (26.596, pari all'86,6\%). Le coppie miste hanno visto il prevalere dei primi matrimoni rispetto alle seconde nozze, sia nel caso in cui si sono sposate in chiesa, sia nel caso in cui hanno contratto matrimonio con rito civile.

Resta da dire che, tra il 2010 e il 2012, le nozze con sposi entrambi stranieri sono cresciute poco più di quelle in cui almeno uno dei due coniugi è nato all'estero $(15,7 \%)$. Questa tipologia di matrimoni rimane comunque minoritaria (4,8\% sul totale delle celebrazioni) e, all'interno delle circa 10.000 unioni complessive, le più numerose sono quelle fra romeni, seguite dai matrimoni tra cinesi, fra nigeriani, tra cittadini della Moldavia, fra ucraini e tra peruviani. Meno frequenti sono le coppie composte da entrambi i coniugi provenienti dall'Albania o dal Marocco, pur essendo tali nazionalità immigrate tra le più consistenti in Italia. Motivi diversi spingono verso queste attitudini concernenti la nuzialità - e certo hanno a che fare con le differenti propensioni culturali di ciascuna comunità interessata, ma s'intrecciano anche con progettualità ed esperienze migratorie specifiche.

Comunque sia, in Italia i matrimoni misti sono in continuo aumento e ciò è anzitutto dovuto al fatto che nel nostro Paese il fenomeno migratorio ha ormai assunto forme strutturali. Si tratta di un processo che, del resto, ha investito un numero sempre più elevato di Paesi europei, in cui la presenza migratoria ha determinato un'evoluzione sia dei modelli famigliari sia nelle forme di unione tra coniugi. Sul nostro territorio nazionale, mentre nel corso del tempo i comportamenti matrimoniali hanno subito cambiamenti anche tra gli italiani, le coppie miste hanno introdotto nuovi modi con cui si formano e si ricompongono i nuclei famigliari. Si tratta ovviamente di mutamenti che rinviano a più profonde trasformazioni sociali e culturali che stanno attraversando il mondo contemporaneo, ma che trovano nei dinamismi migratori una delle più rilevanti modalità di espressione, capace di proiettare ricadute significative su differenti aspetti del vivere collettivo.

Intanto, la stabilizzazione dell'immigrazione genera effetti a catena e, nel lungo periodo, è in grado di incidere sull'organizzazione territoriale e sulla riproduzione sociale del Paese che li accoglie. E già oggi, anche se nella società italiana gli stereotipi e certi pregiudizi tendono a perdurare, i migranti occupano un posto importante nella nostra economia nazionale, interpretano un ruolo non di secondo piano nell'apportare stimoli culturali, svolgono una funzione demografica strategica nell'arginare l'invecchiamento della popolazione e, in futuro, avranno un sempre maggiore impatto nel sostenere il sistema pensionistico. Del resto, per rimanere al caso delle unioni miste, in esse la propensione procreativa è assai più elevata che tra le coppie con entrambi i genitori italiani; nel mondo della scuola, poi, aumenta gradualmente la presenza di figli nati da matrimoni misti; infine, le famiglie con alme- 
no un genitore straniero e le seconde generazioni stanno assumendo un peso ineludibile nei percorsi d'integrazione degli immigrati.

Tutti questi fattori contribuiscono a collocare le coppie miste in un orizzonte di primo piano nei processi di trasformazione sociale e il loro protagonismo, per quanto riguarda l'affermarsi di nuovi modelli famigliari, non si discosta dall'evoluzione più generale. Anche tra di esse, infatti, crescono le unioni celebrate con rito civile, aumenta l'instabilità coniugale benché in misura inferiore rispetto alle famiglie italiane, si conferma la maggiore fragilità economica delle donne nel ruolo di genitore solo in caso di separazione o divorzio. E, nonostante gli elementi di convenienza nelle procedure per l'acquisizione della cittadinanza o l'esistenza dei cosiddetti "matrimoni di riserva", vale a dire sia quelli contratti dopo un'altra esperienza coniugale sia quelli che per problemi legati alla famiglia dei genitori avvengono in tarda età rispetto alla media, le unioni miste hanno comunque un potere dirompente.

Basti pensare al fatto che, in diverso modo, i partner sfidano implicitamente o esplicitamente le "norme" delle società alle quali appartengono, spesso mettono in questione le regole coniugali delle famiglie d'origine, sono costretti a interrogarsi su credenze religiose e schemi culturali ingenuamente ritenuti stabili dai più: insomma, devono confrontarsi con i dinamismi della propria identità individuale e collettiva, rinegoziando continuamente un patrimonio di esperienze e di convinzioni sottoposte a sollecitazioni inaggirabili. È precisamente questo, però, che fa delle coppie miste una componente importante della "diversità", una risorsa ineliminabile per il futuro di una società multiculturale aperta all'innovazione e non irretita negli stilemi consolidati della tradizione.

Le cosiddette seconde generazioni costituiscono un universo composito all'interno del quale si inseriscono anche i figli di questi nuovi nuclei famigliari. Coppie confluite in una popolazione di migranti che già di per sé, come si è avuto modo di vedere, ha un'età mediamente inferiore a quella dei cittadini italiani. In particolare i giovani d'origine immigrata, che nel nostro Paese vivono in una situazione mobile tra assegnazione di ruoli e rivendicazione di diritti, sono difficili da collocare. Diverse espressioni tentano di rubricare la loro posizione: "stranieri non immigrati", "italiani con permesso di soggiorno", "giovani cittadini immigrati", "generazione di italiani d'origine straniera". Dato che i nomi non sono mai innocenti, questo imbarazzo definitorio rivela per un verso la difficoltà di metabolizzare il fenomeno e, per altro verso, l'ambigua condizione nella quale vengono a trovarsi le seconde generazioni nel nostro Paese (Gaffuri, 2013, pp. 159 ss.). Un tema scomodo che, intrecciato con gli ostacoli frapposti al riconoscimento dello status di cittadini ai figli dei migranti, interroga il corpo sociale e le sue istituzioni su quale sia l'idea d'identità nazionale perseguita, vista l'impor- 
tanza assegnata dalla legislazione italiana allo ius sanguinis per l'attribuzione della cittadinanza.

Anche sul piano statistico si riscontrano difficoltà metodologiche a identificare e quantificare con precisione le seconde generazioni, una categoria le cui implicazioni sociali sono diversificate e cruciali per il futuro di una collettività demograficamente in affanno. Intanto, classificare i giovani d'origine straniera nati in Italia a partire dalla nazionalità dei loro genitori significa gettare le basi per un trattamento giuridico differenziato - e ciò vale anche per i minorenni arrivati nella penisola tramite il ricongiungimento famigliare. In secondo luogo, la questione è spinosa perché il senso comune, la comunicazione mediatica e ampi strati dell'opinione pubblica tendono a definire questi giovani come immigrati a tutto tondo, senza dare peso adeguato alle discriminazioni che subiscono.

Eppure, tenendo conto delle potenzialità racchiuse nella capacità delle seconde generazioni di rifiutare la loro condizione d'italiani con permesso di soggiorno, cioè di negare la propria inclusione differenziale nel tessuto sociale d'approdo, si può gettare nuova luce sulla funzione strategica di questi giovani. I figli di cittadini stranieri hanno infatti un ruolo importante per il domani della nostra società e già oggi manifestano posizioni critiche. Se non venissero considerati solo come una "posterità inopportuna" (Queirolo Palmas, 2012, pp. 103 ss.) e li si guardasse in termini positivi, oltre a contribuire al tasso di natalità potrebbero diventare protagonisti di innovativi percorsi di trasformazione sociale, insieme ai giovani italiani loro coetanei.

Inquadrare in una prospettiva generazionale la questione dei minorenni d'origine straniera significa sottolineare quel sincronismo delle relazioni tra persone della stessa età che permette di elaborare un'identità multipla e "tattica", legata alla dimensione transnazionale e all'appartenenza plurale (Colombo, 2012, pp. 99 ss.). Nati in tempi dominati da spazi globali nei quali si verificano ricomposizioni che assumono forme differenti secondo le tappe del ciclo di vita, essi riescono più facilmente a bilanciare l'ansietà di assimilazione della società d'accoglienza. E ciò è possibile anche perché, tra i figli scolarizzati degli immigrati, esistono nei fatti dinamismi interculturali quotidiani. Così, la coabitazione e la condivisione creano una "dissonanza generazionale" che si manifesta nel diverso grado di esposizione dei giovani alla cultura del Paese ospitante rispetto ai propri genitori (processo richiamato in Ambrosini e Caneva, 2012, pp. 127 ss.).

Dopo i percorsi formativi nelle scuole primarie e secondarie inferiori, le superiori sono caratterizzate dalla fase dell'adolescenza, un momento assai delicato soprattutto per coloro che sono arrivati con i ricongiungimenti famigliari. In più, la scolarizzazione è un fattore che può esercitare un'influenza positiva o negativa sui minori d'origine straniera, secondo l'età del loro arrivo in Italia, l'appartenenza dei genitori a una certa classe sociale, 
la capacità delle istituzioni scolastiche di promuovere l'inclusione. E proprio focalizzando l'attenzione sugli adolescenti "stranieri" emergono nodi sociali e culturali rivelatori, poiché i giovani sono più sensibili alle forme della globalizzazione contemporanea che hanno diretto rapporto con i processi d'integrazione locale. In questo orizzonte, la filiazione migrante come elemento identitario delle seconde generazioni rimane un aspetto problematico, al quale si aggiunge quello del lungo percorso cui sono ancora costrette per acquisire la cittadinanza italiana come condizione d'esercizio del "diritto di avere diritti". Ma è il territorio a essere fondamentale nelle politiche d'integrazione, perché l'avvenire di una società aperta in Italia dipenderà dalla capacità dei luoghi di farsi domesticare da altre pratiche d'uso, derivanti dalla presenza sincronica nello stesso spazio geografico di più culture in relazione tra loro. E una polis alfine giusta, in cui il territorio garantisse un accesso più ampio ai beni e ai servizi, agli spazi, ai simboli e ai modi d'abitare, rappresenterebbe un'autentica garanzia per tutti i cittadini, compresi gli ultimi venuti. 


\title{
3. II tutore della convivenza \\ e la progettualità interculturale nei contesti urbani e formativi
}

\author{
di Sergio Bontempelli
}

\subsection{La "città multiculturale" tra realtà e mito}

È ormai divenuto un luogo comune affermare che, a seguito dei flussi migratori degli ultimi decenni, le città italiane siano divenute sempre più multiculturali e multietniche. $E$ in effetti sarebbe difficile sopravvalutare la portata delle trasformazioni - economiche, sociali, culturali, persino urbanistiche e demografiche - che la presenza dei migranti ha apportato nelle realtà urbane del nostro Paese.

Tuttavia, mentre nella letteratura scientifica queste trasformazioni sono state attentamente analizzate nella loro portata e nelle loro conseguen$\mathrm{ze}^{1}$, il discorso pubblico si è spesso nutrito di stereotipi esotizzanti e banali: così, la presenza straniera è stata ridotta ad una improbabile "invasione delle moschee", o al presunto "degrado" dei quartieri maggiormente abitati o frequentati dai migranti, o - ancora - al commercio di prodotti cosiddetti "etnici" come il kebab (a loro volta dipinti come altrettante minacce alla "tradizione italiana"). In questo modo, l'impatto delle migrazioni sulle realtà urbane è stato descritto a partire da un canovaccio narrativo tutto incentrato sulla dicotomia "identità/diversità": un corpo sociale immaginato come omogeneo o comunque unitario (l'Italia, la sua cultura, le sue tradizioni) sarebbe stato attraversato negli ultimi decenni da flussi migratori portatori di diversità culturali più o meno irriducibili.

Anche nei casi, tutt'altro che infrequenti, in cui questo accento sulle differenze assume un valore positivo - quando cioè l'Alterità degli immigrati

1. I testi sono ormai numerosi, ed è impossibile proporre in questa sede una bibliografia completa. Per un quadro di insieme si vedano almeno: Colombo, Genovese e Canevaro (2006); Caponio (2006). Per considerazioni più aggiornate si veda almeno Ambrosini (2014, in particolare capitolo 4, "I multiculturalismi urbani alla prova. Alla ricerca di nuovi linguaggi", pp. 115-142). 
viene presentata come un indispensabile arricchimento per le società ospitanti - questo discorso rischia di occultare alcune evidenze, messe in luce con chiarezza nella letteratura specialistica: qui, per motivi di spazio, possiamo riassumerle solo in modo molto schematico (il lettore perdonerà qualche inevitabile semplificazione).

In primo luogo, il nostro Paese non è, e non è mai stato, culturalmente omogeneo e coeso. La storia italiana - come ogni altra storia nazionale - è anzitutto storia di differenze: differenze religiose (si pensi a minoranze storiche come gli ebrei o i protestanti), geografiche (tra Nord e Sud, tra campagna e città, tra regioni ecc.), culturali, politiche, sociali, di classe, di genere ecc. In altre parole, la cosiddetta "città multiculturale" non è affatto il prodotto delle recenti migrazioni: queste ultime hanno, per così dire, aggiunto ulteriori apporti a un panorama urbano che è da sempre culturalmente variegato, differenziato e segmentato.

In secondo luogo, l'enfasi sulle differenze attribuite agli immigrati rischia spesso di essere eccessiva e fuorviante. Così, solo per fare un esempio, gli stranieri residenti in Italia sono in maggioranza di fede cristiana (cattolica, ortodossa o protestante), un dato che smentisce i timori diffusi di una "islamizzazione" del Paese (IDOS, 2014, pp. 190-198). D'altra parte, la stessa rappresentazione dell'Islam come di un universo radicalmente altro rispetto all'Europa occulta una storia millenaria di scambi, di osmosi e di contatti tra i due mondi²: per tacere poi della pluralità e delle ampie articolazioni interne che caratterizzano tanto il mondo musulmano quanto quello cristiano, irriducibili a "identità" omogenee e indifferenziate.

\subsection{Differenze culturali e asimmetrie di potere}

Il discorso pubblico sull'immigrazione nelle realtà urbane ha insomma avallato l'idea di un conflitto tra culture, e di un connesso problema di convivenza, entrambi indotti dalla diversità di cui i migranti sarebbero portatori ${ }^{3}$.

Si è così creata quella che potremmo definire una retorica della convivenza tra culture: chi si batte per l'inserimento sociale degli immigrati insiste sull'arricchimento reciproco derivante dal contatto tra universi culturali differenti, e vede nella conoscenza, nello scambio e nel mutuo riconoscimento altrettanti antidoti al conflitto. Le parole di Tahar Ben Jelloun sintetizza-

2. Anche in questo caso, il tema è così complesso, e gli studi sono così numerosi, che è impossibile fornire una bibliografia esaustiva, o anche solo indicativa. Qui, per gli aspetti che ci interessano, si veda almeno Campanini, Merzan (2007).

3. Per una critica documentata dell'idea di diversità culturale nei contesti sociali contemporanei si vedano le considerazioni contenute in Aime (2004). 
no con esemplare chiarezza questo approccio: «È l'ignoranza ad alimentare la paura [...]. Guarda per esempio i nostri vicini di casa. Per molto tempo si sono mostrati diffidenti verso di noi, fino al giorno in cui li abbiamo invitati a mangiare il cuscus [...]. Imparare a conoscersi, a parlarsi [...], è questo che potrebbe far regredire il razzismo» (Ben Jelloun, 1998, pp. 17-18).

Gli studi più recenti sui conflitti urbani connessi alle migrazioni ${ }^{4}$ mostrano però una realtà ben più complessa, e sicuramente meno tranquillizzante: il razzismo non deriva dall'ignoranza, e ha poco a che fare con la diversità culturale - spesso più immaginata che empiricamente constatabile - di cui i migranti sarebbero portatori. Non basta un cuscus o una "cena etnica", dunque, per far regredire i fenomeni di xenofobia.

Oggi, gli immigrati vivono situazioni diffuse di discriminazione, esclusione e disparità di trattamento (cfr. Saraceno, Sartor e Sciortino, 2013). In un contesto di crisi economica, poi, il discorso pubblico e politico enfatizza spesso la competizione tra stranieri e autoctoni per l'accesso a risorse di welfare sempre più scarse: alloggi popolari, prestazioni sanitarie, sussidi alle famiglie indigenti e così via. L'immigrato è divenuto in tal modo il facile capro espiatorio di rabbie e paure diffuse: ed è sempre più spesso vittima di stigmatizzazione, quando non di vere e proprie aggressioni a sfondo razziale 5 .

I cosiddetti "problemi di convivenza" sono ascrivibili più spesso a queste asimmetrie di potere che a una generica diversità culturale: la discriminazione da un lato, la competizione tra gruppi sociali etnicizzati dall'altro, innescano e alimentano molti conflitti urbani: gli esempi tratti dalle vicende di attualità - si pensi ai fatti di Tor Sapienza a Roma - sono ormai sempre più numerosi.

Intervenire nei contesti di conflitto urbano, favorire la pacifica convivenza tra cittadini italiani e stranieri immigrati, significa dunque agire su queste asimmetrie di potere, favorire l'empowerment dei gruppi più svantaggiati, e al contempo decostruire pregiudizi e stereotipi diffusi nel corpo sociale. Il compito non è facile, ma non esistono scorciatoie: parafrasando una blasonata definizione, potremmo dire che il contrasto al razzismo "non è un pranzo di gala".

\subsection{Mediare tra diseguali: l'esperienza di Ricostruireln- sieme nel territorio dell'Aquila}

Le vicende accadute nel territorio aquilano all'indomani del sisma del 6 aprile 2009 consentono di illustrare questi fenomeni a partire da un esempio concreto.

4. Per il caso italiano, si vedano almeno: Dal Lago (2004), Cotesta (1999), Zanfrini, (2004 , 2004b), Colombo (2012).

5. Si veda, per gli episodi più recenti, l'ampio dossier di Lunaria (2014). 
Le drammatiche esperienze vissute da un'intera comunità locale - il terremoto, la distruzione del tessuto urbano, poi le tendopoli, la vita da sfollati, le polemiche sulla gestione autoritaria della Protezione Civile, i ritardi nella ricostruzione - hanno fatto emergere inedite forme di solidarietà e nuovi legami sociali tra cittadini (Spila, 2009). E tuttavia, questa solidarietà spontanea, spesso generosa, non ha prodotto automaticamente legami di uguaglianza e di parità: già all'indomani del sisma, nelle tendopoli il clivage italiani/stranieri era ben visibile sia nella gestione istituzionale dei campi (nei quali, in molti casi, i migranti privi di residenza o di permesso di soggiorno non potevano entrare), sia nelle relazioni tra gli ospiti. Da una ricerca pubblicata nel 2010 emergono numerosi casi di discriminazione, di intolleranza o di vero e proprio razzismo contro i migranti accolti nelle tendopoli (cfr. Vaccarelli, 2010).

In questo contesto un gruppo di associazioni - composto da ARCI, Caritas diocesana e da altre realtà attive nel contesto aquilano - ha dato vita al Coordinamento RicostruireInsieme, finalizzato a promuovere la convivenza e il dialogo interculturale nel tessuto sempre più lacerato della città. Sin dai suoi primi passi, il coordinamento ha intuito però che i conflitti tra italiani e stranieri dovevano essere letti non in chiave "culturalista", ma a partire dalle discriminazioni e dalle "asimmetrie di potere". Garantire il pieno accesso dei migranti nelle tendopoli, favorire il rilascio e il rinnovo dei permessi di soggiorno, costituire dei punti informativi contro le discriminazioni sono state le prime azioni a supporto dei migranti. L'ottica era quella di promuovere il dialogo garantendo diritti: nessuna concessione, dunque, a quella che qui sopra abbiamo definito "retorica della convivenza tra culture".

\subsection{Il tutore della convivenza e il rapporto tra università e città}

Questa prospettiva si è alla fine concretizzata nella definizione di una nuova figura professionale, quella del tutore della convivenza, su cui vale la pena spendere qualche parola conclusiva.

Il tutore della convivenza è un operatore capace di intervenire in situazioni di conflitto tra cittadini italiani e migranti, per favorire il dialogo, agevolare la comunicazione tra le parti e contrastare le discriminazioni. Benché chiamato di fatto a mediare, il tutore non è però una figura imparziale: consapevole delle asimmetrie di potere, è chiamato a colmarle, supportando la parte più debole e operando per il suo pieno accesso ai diritti. Al contempo, il tutore lavora per contrastare stereotipi e pregiudizi che impediscono la comunicazione e che irrigidiscono il conflitto. 
Su questa prospettiva, nel contesto cittadino aquilano e nell'ambito del progetto "Unidiversità", si è costruito il percorso formativo della nuova figura professionale. Nel corso di formazione per tutori della convivenza conclusosi nell'aprile 2015 - si sono proposti moduli sulla costruzione sociale dello straniero, sul razzismo, sulla segregazione urbana e spaziale dei migranti' ${ }^{6}$ l'ottica è quella di restituire l'idea del razzismo come costruzione complessa e multidimensionale (contrastando letture semplicistiche e tranquillizzanti, come quella del "razzismo frutto dell'ignoranza"). Tra le competenze richieste vi sono anche quelle giuridiche, necessarie per la tutela e la promozione dei diritti: i futuri tutori della convivenza hanno seguito moduli sul contrasto alle discriminazioni e sullo status giuridico dello straniero. Infine, particolare attenzione è stata dedicata allo studio dei fenomeni migratori, restituendo la loro dimensione storica e sociale.

Il corso di formazione è stato organizzato assieme all'università: la scelta è stata quella di coinvolgere le istituzioni formative e di ricerca in un percorso che per sua natura richiede un incrocio costante di saperi accademici (storici, sociologici, giuridici) e saperi "applicativi", tipici della cosiddetta street level bureucracy.

$\mathrm{Al}$ termine del percorso formativo, il tutore della convivenza ̀̀ - per così dire - pronto ad entrare in azione. Si tratterà, nei prossimi anni, di valutare l'efficacia e i limiti di questa nuova figura professionale.

6. Si veda anche il capitolo 15. 


\title{
4. L'università nel processo di internazionalizzazione e mobilità globale: obiettivi, risultati e sfide
}

\author{
di Anna Tozzi
}

\subsection{II sistema Italia di fronte alle sfide europee e globali}

Negli ultimi venti anni l'umanità ha sperimentato profondi e rapidi cambiamenti culturali, sociali ed economici, mai osservati prima nel corso dei secoli e in alcuni casi assolutamente impensabili.

Se andiamo indietro al 1990 lo scenario che affiora alla memoria sembra ora quasi incredibile:

- fino al 1990 l'Europa era nettamente divisa in due;

- i contatti tra Est e Ovest erano molto difficili;

- il mondo era completamente diverso: I'uso dei computer era solo all'inizio, gli studenti usavano ancora i libri, vocabolari, enciclopedie e frequentavano le biblioteche, la comunicazione avveniva solo per telefono (fisso) e per posta, il fax era uno strumento "sofisticato";

- la formazione, anche quella universitaria, era di carattere "nazionale";

- il programma Erasmus era appena stato lanciato (1987).

$\grave{E}$ in questo contesto che il 16 novembre 1989 avviene la "caduta del muro di Berlino" che in effetti ha rappresentato la fine della Cortina di ferro e la caduta di altri muri più o meno manifesti.

L'Europa è chiamata a dare rapide risposte al nuovo assetto politico-sociale. Si evidenzia la necessità di sostenere la riforma dell'istruzione formazione dell'Europa centrale e orientale. L'università, per la sua natura interdisciplinare, e la sua influenza multi settoriale diventa uno degli attori chiave per gestire il cambiamento. A dicembre 1989, un mese dopo la caduta del muro, a Strasburgo vengono stabiliti i criteri e i fondi per realizzare il programma:

- Ia CE lancia il programma TEMPUS I che subito dimostra di essere uno strumento eccellente per "riunire" l'Europa; 
- specifici fondi PHARE vengono messi a disposizione per aiutare e sostenere la riunificazione della Germania, I'Ungheria, la Polonia, la Repubblica Ceca, la Romania, la Bulgaria e quella che a breve non sarà più la Jugoslavia;

- 12 Nazioni europee collaborano con l'Europa centrale e orientale nella rapida trasformazione.

Già nel 1993 l'Europa ha stabilizzato il suo nuovo assetto e si prepara al primo allargamento dei suoi confini. Quasi tutti i nuovi Stati dell'Est Europa partecipano all'Erasmus, la collaborazione a livello universitario identifica chiaramente i nuovi target. In questo scenario vengono lanciati i programmi Socrates e Leonardo da Vinci, che vedono la collaborazione nel settore della scuola e della formazione superiore degli Stati Membri e i 10 Paesi dell'Europa centrale e orientale, Cipro, e Malta. L'Europa ha nuovi confini, e viene lanciato il TEMPUS II (1994-1999) e ai fondi PHARE si aggiungono i fondi TACIS. I Paesi target della cooperazione passano da 11 a 26 :

- la Bielorussia, l'Ucraina e la Federazione Russa si uniscono al Programma già nel 1993;

- il Kazakistan, il Kirghizistan e l'Uzbekistan si uniscono nel 1994, l'Armenia, l'Azerbaijan e la Georgia nel 1995, seguiti dal Tagikistan nel 1996 e dal Turkmenistan nel 1997;

- la Mongolia partecipa dal 1995;

- l'Europa effettua un altro allargamento: nel 1995 l'Austria, la Svezia e la Finlandia diventano membri dell'UE che ora conta 15 Stati.

Una delle attività finanziate era la mobilità di studenti verso atenei europei estendendo quanto succedeva con l'Erasmus per la mobilità intereuropea. Le classi universitarie vedono l'arrivo di studenti provenienti da vari Paesi non europei che sperimentano le stesse difficoltà legate alla mobilità di breve durata sostenuta dall'Erasmus: mancato riconoscimento degli esa$\mathrm{mi}$, traduzione dei voti nel sistema locale inadeguato.

Il 1999 rappresenta un anno cruciale per l'assetto dei sistemi universitari: la firma della Dichiarazione di Bologna, che lancia la grande sfida di creare uno Spazio Europeo della Formazione Superiore (EHEA - European Higher Education Area) per permettere la mobilità di diplomati e laureati tra diverse istituzioni di diverse Nazioni dell'area rendendo i titoli e le lauree più compatibili ${ }^{1}$. Nel decennio 2000-2010 si assiste ad una grande tra-

1. «The overarching aim of the Bologna Process is to create a European Higher Education Area (EHEA) based on international cooperation and academic exchange that is attractive to European students and staff as well as to students and staff from other parts of the world». 
sformazione dell'organizzazione della formazione superiore, che va sotto il nome di Processo di Bologna, che porterà a riforme dei sistemi universitari non solo nei Paesi dell'Unione ma anche in tutti i Paesi partner della cooperazione. Infatti, come osservato da più parti (Pépin, 2006), Bologna Declaration ha cambiato la prospettiva: non si tratta più solo di mobilità e cooperazione, ma piuttosto di convergenza di sistemi.

Il 2000 è l'anno di avvio di un'altra strategia europea, la Strategia di Lisbona, che intende promuovere lo sviluppo economico, la crescita di posti di lavoro e una migliore coesione sociale creando e supportando l'economia della conoscenza ${ }^{2}$. Di nuovo l'istruzione e la formazione sono gli strumenti fondamentali e la necessità degli Stati Membri di cooperare per creare un metodo di "convergenza" dei sistemi di istruzione dà l'avvio a grandi dibattiti che non si limitano ai confini geopolitici dell'Unione ma che si estendono a tutti i Paesi confinanti.

Nel 2004 si completa il quinto allargamento dell'Unione che include otto Nazioni dell'Europa centrale e orientale e le due isole del Mediterraneo. Il nuovo assetto porta naturalmente la necessità di estendere la cooperazione ai Paesi confinanti con il Sud dell'Europa, cioè i Paesi che si affacciano alla riva sud del Mediterraneo e quindi:

- viene lanciato il TEMPUS III (2000-2007) che vede come Paesi target della cooperazione i Paesi dei Balcani Occidentali (Albania, Bosnia ed Erzegovina), la Croazia, la Repubblica Federale della Jugoslavia e l'ex Repubblica Jugoslava di Macedonia;

- si aggiungono i Paesi mediterranei (Algeria, Egitto, Giordania, Libano, Marocco, i Territori Occupati della Palestina, Siria e Tunisia);

- 10 Paesi prima target di cooperazione diventano Stati Membri e continuano a partecipare al TEMPUS nel ruolo di finanziatori;

- contemporaneamente in Europa si accelera il processo di convergenza e riconoscibilità dei titoli, iniziano i primi corsi di laurea internazionali con titoli doppi/congiunti;

- tutti i Paesi membri riformano il sistema della formazione superiore individuando i 3 cicli: Bachelor, Master, PhD, che in Italia sono Laurea, Laurea Magistrale e Dottorato;

- Socrates II, con i sottoprogrammi relativi a tutti i livelli di formazione, continua ad essere un programma di grande successo e strumento fondamentale per la realizzazione degli obiettivi.

Durante il settennio 2000-2007 iniziano i primi segnali della incombente crisi economica. Si assiste ad un aumento continuo di flussi migrato-

2. Si consulti il sito web www.eea.europa.eu/policy-documents/lisbon-european-council23-and riguardo la Lisbon European Council Presidency Conclusions del 23 e 24 marzo 2000 . 
ri da varie parti del mondo afflitte da guerre, conflitti e crisi di vario genere. L'Europa è meta di persone in cerca di una vita migliore, ma anche di studenti che riconoscono l'importanza e la validità del sistema universitario europeo che acquista sempre più una sua caratteristica e visibilità. Il Processo di Bologna raffina i suoi obiettivi, mette al centro la necessità di "assicurare la qualità" dei suoi percorsi formativi. La mobilità, l'integrazione e la convergenza dei titoli non possono realizzarsi senza la garanzia della qualità della formazione a tutti i livelli. I Paesi target della cooperazione, molti dei quali hanno sottoscritto la Bologna Declaration, vengono coinvolti nel Processo. La cooperazione riguarda la progettazione dei curricula, la gestione e democratizzazione degli istituiti e dei sistemi di formazione. Come osserva Janez Potočnik, Commissario per la Scienza e Ricerca nel 2006 le università sono le «power-houses of knowledge generation but they needed to adapt to the demands of a global, knowledge-based economy» ${ }^{3}$.

Si arriva così alla programmazione 2007-2013 in cui grande rilievo e importanza continua ad avere la cooperazione nell'istruzione e nella formazione. Il Programma LLP (Lifelong Learning Programme) attualmente raggruppa vari programmi di mobilità come Erasmus, Leonardo, Comenius e TEMPUS che sono riconosciuti fondamentali per la creazione dello Spazio Europeo della Formazione Superiore e per la risposta alle nuove sfide economiche e sociali che si apprestano ad affrontare il mondo. Quindi all'interno di tale programmazione:

- viene lanciato il TEMPUS IV che amplia ancora di più il suo target geografico ma sostanzialmente continua le sue azioni di successo: approccio bottom-up, trasferimento di conoscenze e know-how, collaborazione tra istituzioni e persone;

- viene lanciato il programma innovativo Erasmus Mundus, che premia e valorizza le iniziative di curricula congiunti europei (Erasmus Mundus Master Course) sostenendo economicamente la mobilità dei migliori studenti del mondo presso le istituzioni europee titolari dei prestigiosi $E M$ Master Degree, e la mobilità del tutto simile a quella del collaudato Erasmus verso istituzioni europee da varie regioni del mondo, non solo quelle target del TEMPUS (Erasmus Mundus Action 2).

Le università attive nella cooperazione vedono le proprie classi integrarsi con studenti che provengono da tutto il mondo. L'internazionalizzazione dei percorsi formativi diventa per alcune università europee l'obiettivo primario delle loro attività. Il riconoscimento dei crediti formativi, l'armonizzazione dei voti, le procedure di iscrizione, i visti e i permessi di

3. European Commission Press Release (RAPID) IP/06/592 del 10.05.2006 sulla modernizzazione delle università europee. 
soggiorno, il dialogo tra amministrazioni, ecc. diventano vorticosamente l'argomento di discussione a livello nazionale e istituzionale. La trasformazione reale è stata molto più veloce di quella organizzativa. Le esperienze fatte diventano la base di partenza della programmazione 2014-2020 che vede il mondo ancora più diverso e i problemi ancora più complessi.

Ma l'idea che il miglioramento del mondo debba per forza passare dalla conoscenza e dalle capacità dei giovani non può cambiare. Le azioni di cooperazione si rafforzano e diventano globali come globali sono i problemi e le sfide economiche e sociali. Il percorso fatto in Europa per la creazione dell'EHEA è seguito e adottato in tutto il mondo. Crediti, workload, mobilità e riconoscimento di titoli ed esami è l'argomento di discussione e accordi di Ministri e Presidenti di tutto il mondo. L'università ha sempre il ruolo fondamentale nel processo come produttore e diffusore di conoscenza.

I nuovi programmi, come Capacity Building (nuova versione del TEMPUS) e Credit Mobility (nuova versione dell'EMA2) sosterranno il cambiamento e la cooperazione nei prossimi anni. Ma l'nternazionalizzazione della formazione superiore, e non solo, è un dato di fatto che può solo crescere.

In tutta questa trasformazione, l'Università degli Studi dell'Aquila è stata sempre presente mediante progetti sia nel TEMPUS che nell'ERAMUS MUNDUS, creando una vasta rete di relazioni affidabili e prestigiose.

L'esperienza inizia con il TEMPUS PHARE nel 1992, con il progetto JEP-2692-92 "Upgrading of education in algebraic and categorical methods in computer science" - target Repubblica Ceca, Ungheria e Slovacchia - che con il progetto poterono aggiornare i laboratori e acquistare i primi computer. E continua negli anni con altri progetti in varie regioni del mondo. Le attività sono varie e riguardano sia l'aggiornamento dei curricula sia l'organizzazione della gestione, in particolare il sostegno alle relazioni internazionali:

- JEP 21117-2000 "Increase of student mobility in the economic field", target Russia;

- JEP 27083-2006 "ICT Curriculum reform in Lisbon perspective", target Russia;

- UM_JEP-27129-2006 (TJ) "Bologna process promotion in Tajikistan through the reorganisation of International Relations Offices" - IRORG, target Tagikistan;

- 145686-TEMPUS-1-2008-FR-TEMPUS-SMHES "Awareness raising, Interest development, desire creation and Action stimulation on the Bologna Process expansion in Central Asian countries and Russia" - AIDA, target Russia, Uzbekistan e Kazakhstan;

- 144976-TEMPUS-1-2008-RO-TEMPUS-JPCR "Professionnalisation des enseignements en travail social", target Russia, Ucraina e Kazakistan; 
- 144935-TEMPUS-1-2008-1-UK-TEMPUS-JPCR "Chemical Engineering: Curriculum Development and International Recognition", target Russia e Kazakistan;

- 144654-TEMPUS-2008-MA-JPGR "Gouvernance Numérique des Universités Marocaines", JPCR-JP-159143-2009 "Using Local Resources for Microregional Development, Sustainable Agribusiness and Tourism in the Southern Balkans" - SATIS, target Albania, Macedonia, Kosovo;

- JPCR-JP-159143-2009 "Development of the E-learning and Distance Learning Courses and Assessment in Biomedical Sciences in the Southern Caucasus";

- 511172-TEMPUS-1-2010-1-DE-JPCR "Curriculum Invoking Bologna-aligned Education Leading to reform in Environmental Studies" - CIBELES, target Tagikistan, Kazakistan, Uzbekistan, Kirghizistan, Turkmenistan e Georgia;

- 510941-TEMPUS-1-2010-1-IL- SMHES "English For All in Academia, to Foster Education, Research and Innovation" - EFA, target Israele.

- 516885-TEMPUS-1-2011-1-ES-TEMPUS-JPHES "Entrepreneuriat et Valorisation de la Recherche" - EVARECH, target Marocco, Tunisia e Algeria;

- 516721-TEMPUS-1-2011-1-RO-TEMPUS-JPCR "Licence-Master professionnels en psychologie sociale pour intervenir auprès des publics migrants en Russie, Ukraine et au Kazakhstan", target Russia, Ukraina, Kazakhstan;

- 517313-TEMPUS-1-2011-1-IT-TEMPUS-JPCR "Environment protecttion through development and application of sustainable Agriculture Technologies" - EPASAT, target Tagikistan, Kazakhstan, Uzbekistan, Kirghizistan.

- 530154-TEMPUS-1-2012-1-IT-TEMPUS-JPGR "Student Support and Development Services" - SSDS, target Kazakhstan, Kyrgyzstan, Israele, Georgia, Azerbaijan;

- 543683-TEMPUS-1-2013-1-IL-TEMPUS-JPCR "English as the Cornerstone of Sustainable Technology and Research - ECOSTAR", target Israele.

- 543713-TEMPUS-1-2013-1-ES-TEMPUS-JPHES "Moderniser la formation sur les Energies Renouvelables (ER) au Maghreb: Transfert de l'expérience UE - MOMATE", target Marocco, Tunisia e Algeria;

- 543802-TEMPUS-1-2013-1-UK-TEMPUS-JP "Establishment of Multidisciplinary Innovative Centres for the Development of Virtual Laboratories (MICVL) in Biology and Medicine - MICVL", target Georgia e Armenia;

- 544046-TEMPUS-1-2013-1-PS-TEMPUS-JPHES "Career-Oriented Curricula Development for Road Vehicle Maintenance - CODE", target Palestina.

Le relazioni costruite durante la realizzazione dei progetti TEMPUS hanno facilitato la realizzazione dei progetti Erasmus Mundus Action 2:

- 2010-2380/001-001-EMA2 "Transfer of Appropriate Requirements for Global Education and Technology" - TARGET (Asia centrale);

- 2011-2569/001-001-EMA2 "Transfer of Appropriate Requirements for Global Education and Technology" - TARGET II (Asia centrale); 
- 2010-2376/-001-EMA2 "Transfer of skills, knowledge and ideas to Central Asia" - TOSCA (Asia centrale);

- 204625-1-2011-1-EMA21 "European Research and education collaboration with Western Balkans" - EUROWEB (Balcani);

- 372296-EM-1-2012-1-EMA21 "Transfer of skills, knowledge and ideas to Central Asia" - TOSCA II (Asia centrale);

- 372244-EM-1-2012-1-AT-EMA21 "Knowledge Management Technology Transfer and Education Programme" - GATE (Sud-Est asiatico);

- 545788-EM-1-2013-1-ES-EMA21 "Intercultural Exchange for Internationalization: Research Management, Student employability and Academic Excellence" - PHOENIX (Medio Oriente);

- 551742-EM-1-2014-1-IT-EMA21, 2014-2018 "IntegrAted Studies for Syrian and eUropean universities" - ASSUR (Siria);

- 552061-EM-1-2014-1-IT-EMA21, 2014-2018 "Mobility As keY factor for quAlity eNhancement of EU and LA universities" - MAYA-NET (America Latina).

- Attraverso questi progetti di mobilità, hanno preso forma le lauree internazionali (GSEEM, Modelli Matematici, Biologia Ambientale, Fisica, ICT, e altri) e l'Erasmus Mundus Master Course: 2013-01000/0012014-2019 "Mathematical Modelling in Engineering: theory, numerics, applications" - MathMods.

Negli ultimi anni le nostre classi si sono popolate di studenti di varie nazionalità. Le diverse culture si sono integrate mediante l'impegno di studio portato avanti insieme agli studenti locali.

È su questo variegato panorama che si inserisce il progetto "Unidiversità" che vuole dare un contributo all'integrazione e una testimonianza su come la cultura e il dialogo siano gli strumenti per una convivenza pacifica. Ma vuole anche esplicitare i problemi, discuterli e analizzarli, per mettere in campo le risorse di ognuno in termini di civiltà, disponibilità, ascolto, pazienza e perché no di difesa delle differenze come "strumenti" per la costruzione di qualcosa di diverso e possibilmente migliore. 


\title{
5. I tempi sono maturi: intercultura all'università
}

\author{
di Alessandro Vaccarelli
}

\subsection{Lo specchio deformante: il paradosso multiculturale della presenza straniera all'università}

La presenza straniera nell'università italiana ha una lunga storia, molto più lunga di quella che possiamo riferire alla scuola, dove un'incidenza significativa di studenti stranieri si è registrata solo a seguito dell'affermarsi delle migrazioni da altri Paesi e continenti.

I dati a disposizione segnalano come già negli anni '50 la presenza di studenti non italiani in ambito accademico superasse la soglia dell' $1 \%$, dato che, se riferito esclusivamente al contesto italiano (e dunque al di fuori di qualsiasi comparazione con altri Paesi), ci sembra piuttosto significativo in rapporto ad almeno tre considerazioni (Tab. 1):

- ancora nel 1981, quando il Censimento della popolazione registra un saldo migratorio positivo che dà il via ufficiale alla nuova stagione della società multiculturale ${ }^{1}$, gli stranieri presenti sul territorio nazionale pesano soltanto per lo $0,37 \%$ sul totale della popolazione (210.937 su oltre 56 milioni di residenti) ${ }^{2}$. Sempre nello stesso anno, la percentuale degli studenti stranieri nelle università italiane si attesta al 2,97\%;

1. Sul multiculturalismo in Italia esiste un dibattito ampio e articolato. Nella letteratura scientifica si fa sempre riferimento al carattere intrinsecamente multiculturale della società italiana, che parte da lontano e che prescinde anche dall'arrivo e dalla presenza di migranti. Con questa consapevolezza parleremo di stagione del multiculturalismo italiano, riferendoci alla fase storica in cui esso viene ulteriormente specificato e declinato, grazie a quei flussi migratori in entrata che hanno strutturalmente trasformato l'assetto sociale, a partire da una nuova idea di diversità culturale e linguistica. Cfr. Fiorucci, 2000; Genovese, 2003.

2. Fonti: Serie storiche ISTAT (http://seriestoriche.istat.it) e Censimento della Popolazione 1981 (www.istat.it/it/files/2011/06/Italia_in_cifre_20132.pdf). 
- bisogna aspettare almeno gli anni '90 per registrare una percentuale di studenti stranieri superiore all' $1 \%$ nella scuola italiana e per percepire tale fenomeno come degno di attenzione da parte delle istituzioni e delle comunità scientifiche che lo hanno poi studiato ed esaminato;

- il numero di studenti stranieri e il loro peso percentuale nelle università italiane tendono a crescere in modo rapido e consistente, con qualche battuta di arresto negli anni '90 e una consistente ripresa nel primo decennio del 2000 (Graf. 1).

Per molti anni dunque, è la stessa presenza di studenti che scelgono di venire a studiare in Italia, a rappresentare una fetta estremamente significativa della presenza straniera in Italia, un fattore importante e relativamente "stabile" di multiculturalismo prima ancora di entrare - a partire dagli anni '80/'90 - nella stagione dello stesso multiculturalismo.

Tab. 1 - Presenza di studenti di cittadinanza non italiana nelle università - Serie storica (1995-2014)

\begin{tabular}{lccc}
\hline A.A. & $\begin{array}{c}\text { Iscritti di } \\
\text { cittadinanza } \\
\text { non italiana }\end{array}$ & Totale iscritti & $\begin{array}{c}\text { \% sul } \\
\text { totale iscritti }\end{array}$ \\
\hline $1955 / 56$ & 2.828 & 210.228 & 1,34 \\
\hline $1961 / 62$ & 3.967 & 287.975 & 1,37 \\
\hline $1965 / 66$ & 6.130 & 404.938 & 1,51 \\
\hline $1971 / 72$ & 17.957 & 759.872 & 2,36 \\
\hline $1975 / 76$ & 18.921 & 935.795 & 2,02 \\
\hline $1981 / 82$ & 30.493 & 1.024 .681 & 2,97 \\
\hline $1985 / 86$ & 26.268 & 1.113 .175 & 2,35 \\
\hline $1991 / 92$ & 20.513 & 1.475 .400 & 1,39 \\
\hline $1995 / 96$ & 21.521 & 1.685 .921 & 1,27 \\
\hline $2001 / 02$ & 25.977 & 1.702 .575 & 1,52 \\
\hline $2005 / 06$ & 41.589 & 1.796 .270 & 2,31 \\
\hline $2011 / 12$ & 66.797 & 1.766 .205 & 3,78 \\
\hline $2013 / 14$ & 70.635 & 1.693 .804 & 4,17 \\
\hline
\end{tabular}

Fonte: Elaborazione su dati ISTAT (Serie storiche) 
Graf. 1 - Presenza di studenti di cittadinanza non italiana nelle università - Serie storica (1955-2014)

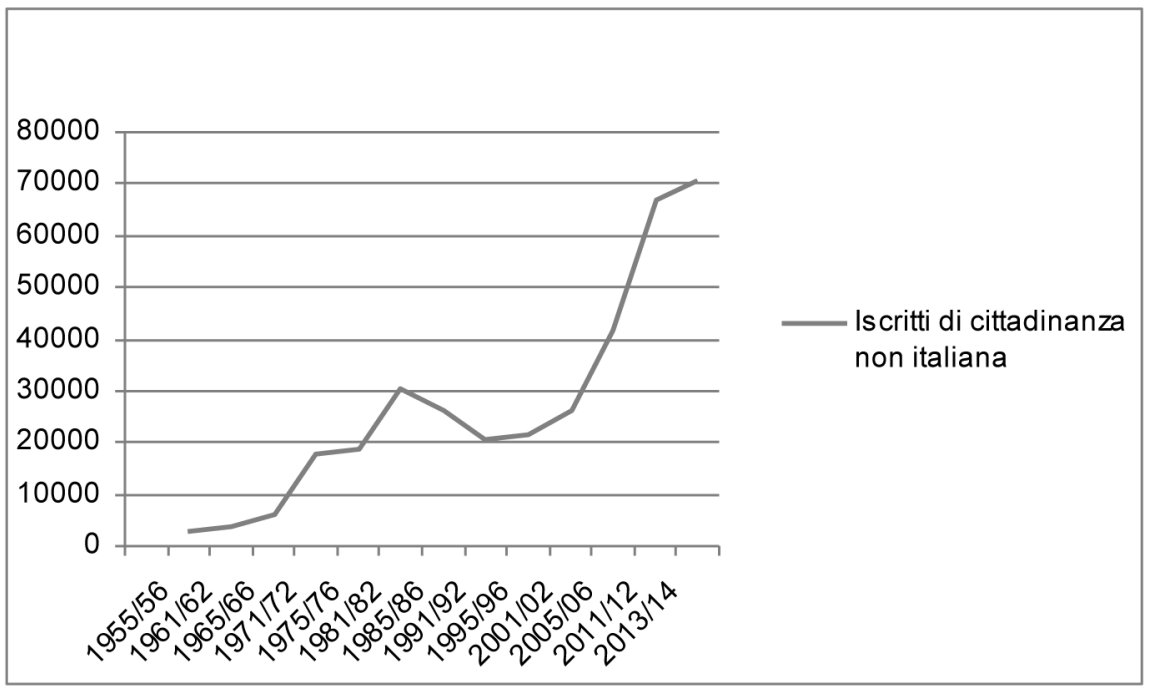

Fonte: Elaborazione su dati ISTAT (Serie storiche)

Dai dati a disposizione (Tab. 2), osserviamo infatti come, ancora nel 1981, la presenza di studenti universitari di cittadinanza non italiana incida addirittura per il 14,45\% sul totale della presenza straniera in Italia, incidenza che già si assottiglia al 5,75\% nel 1991 (anno di applicazione della Legge Martelli), per scendere sotto il 2\% a partire dal 2001.

Tab. 2 - Gli studenti universitari di cittadinanza non italiana e la presenza immigrata in Italia

\begin{tabular}{lcccc}
\hline A.A. & $\begin{array}{c}\text { Iscrittidi } \\
\text { cittadinanza } \\
\text { non italiana }\end{array}$ & $\begin{array}{c}\text { \% sul } \\
\text { totale iscritti }\end{array}$ & $\begin{array}{c}\text { Popolazione } \\
\text { straniera } \\
\text { in Italia }\end{array}$ & $\begin{array}{c}\text { \% studenti su } \\
\text { popolazione } \\
\text { straniera in } \\
\text { Italia }\end{array}$ \\
\hline $1981 / 82$ & 30.493 & 2,97 & 210.937 & 14,45 \\
\hline $1991 / 92$ & 20.513 & 1,39 & 356.159 & 5,75 \\
\hline $2001 / 02$ & 25.977 & 1,52 & 1.334 .889 & 1,94 \\
\hline $2011 / 12$ & 66.797 & 3,78 & 4.570 .317 & 1,46 \\
\hline $2013 / 14$ & 70.635 & 4,17 & 4.922 .085 & 1,43 \\
\hline
\end{tabular}

Fonti: Anagrafe studenti MIUR; Serie storiche ISTAT 
In questo senso l'università si è posta come avanguardia del multiculturalismo italiano, ma non ha saputo interpretare in modo pieno e coerente questo suo carattere dalle forti implicazioni socio-culturali, politiche e, non da ultimo, economiche. Paradossalmente, nel momento in cui l'Italia diventa un Paese di immigrazione, con ritmi di crescita della componente straniera significativi e costanti, la presenza di studenti di cittadinanza non italiana nelle università, per quanto in crescita in termini assoluti e, più lievemente, in termini relativi, sembra sfumare di fronte alla nuova compagine sociale segnata dalla diversità culturale, sia in termini quantitativi sia, come vedremo, per una differenziazione dei profili sociali degli studenti stessi. E mentre, in questi anni, è la scuola che vede il fenomeno farsi avanti in modo costante e progressivo, l'università sembra passare in retroguardia rispetto alle presenze straniere se considerate in termini relativi. La Tabella 3 ci permette infatti di osservare come il numero assoluto

Tab. 3 - Studenti di cittadinanza non italiana iscritti a scuola e all'università - Valori assoluti e per 1000 iscritti

\begin{tabular}{lccccc}
\hline $\begin{array}{l}\text { A.S. } \\
\text { A.A. }\end{array}$ & $\begin{array}{c}\text { Scuola } \\
\text { (tutti gli } \\
\text { ordini) }\end{array}$ & $\begin{array}{c}\text { Scuola } \\
\text { Secondaria } \\
\text { di Il grado }\end{array}$ & $\begin{array}{c}\text { Per 1000 } \\
\text { iscritti }\end{array}$ & Università & $\begin{array}{c}\text { Per 1000 } \\
\text { iscritti }\end{array}$ \\
\hline $1993 / 94$ & 33.081 & 5.324 & 1,9 & 23.623 & 14,5 \\
\hline $1994 / 95$ & 35.284 & 6.060 & 2,2 & 22.918 & 13,8 \\
\hline $1995 / 96$ & 39.872 & 6.410 & 2,4 & 21.521 & 12,8 \\
\hline $1996 / 97$ & 44.996 & 7.837 & 3,0 & 22.988 & 13,0 \\
\hline $1997 / 98$ & 57.299 & 9.280 & 3,6 & 24.010 & 13,6 \\
\hline $1998 / 99$ & 73.222 & 10.520 & 4,1 & 23.063 & 13,8 \\
\hline $1999 / 00$ & 101.240 & 14.801 & 5,8 & 23.666 & 10,0 \\
\hline $2000 / 01$ & 127.916 & 21.149 & 8,2 & 25.769 & 15,3 \\
\hline $2001 / 02$ & 156.883 & 26.963 & 10,4 & 25.977 & 15,3 \\
\hline $2002 / 03$ & 192.298 & 34.890 & 13,3 & 31.778 & 18,0 \\
\hline $2003 / 04$ & 247.641 & 52.380 & 19,9 & 35.299 & 21,2 \\
\hline $2004 / 05$ & 296.455 & 63.833 & 24,0 & 38.298 & 21,3 \\
\hline $2005 / 06$ & 347.153 & 83.052 & 30,9 & 41.589 & 22,8 \\
\hline $2006 / 07$ & 407.166 & 103.287 & 37,8 & 47.506 & 26,3 \\
\hline $2007 / 08$ & 463.632 & 119.520 & 43,5 & 51.790 & 28,6 \\
\hline $2008 / 09$ & 504.857 & 130.601 & 48,0 & 55.743 & 31,0 \\
\hline $2009 / 10$ & 565.635 & 153.423 & 57,6 & 58.940 & 33,1 \\
\hline$F 0 n$ & 150 & & &
\end{tabular}

Fonte: Anagrafe studenti MIUR; MIUR, ISMU 
e il valore relativo su 1000 iscritti degli studenti di cittadinanza non italiana nelle scuole (figli delle migrazioni internazionali), segua un incremento significativo, di fronte al quale l'università non tiene il passo: nell'arco di nemmeno un ventennio, dal 1993 al 2009, la scuola secondaria passa da un valore assoluto di 5.324 presenze (1,9 studenti stranieri su 1.000) a 153.000 (57,6 studenti stranieri su 1.000); diversamente, l'università passa da 23.000 presenze $(14,4$ studenti su 1.000$)$ a 58.940 (33,1 studenti su 1000). Il sorpasso della scuola, che registriamo nel 2001/02, è seguito, negli anni successivi, da una sua rapidissima trasformazione in senso multiculturale, come è evidente nel Grafico 2.

Graf. 2 - Confronto studenti stranieri iscritti nella scuola secondaria di II grado e all'università - Serie storica

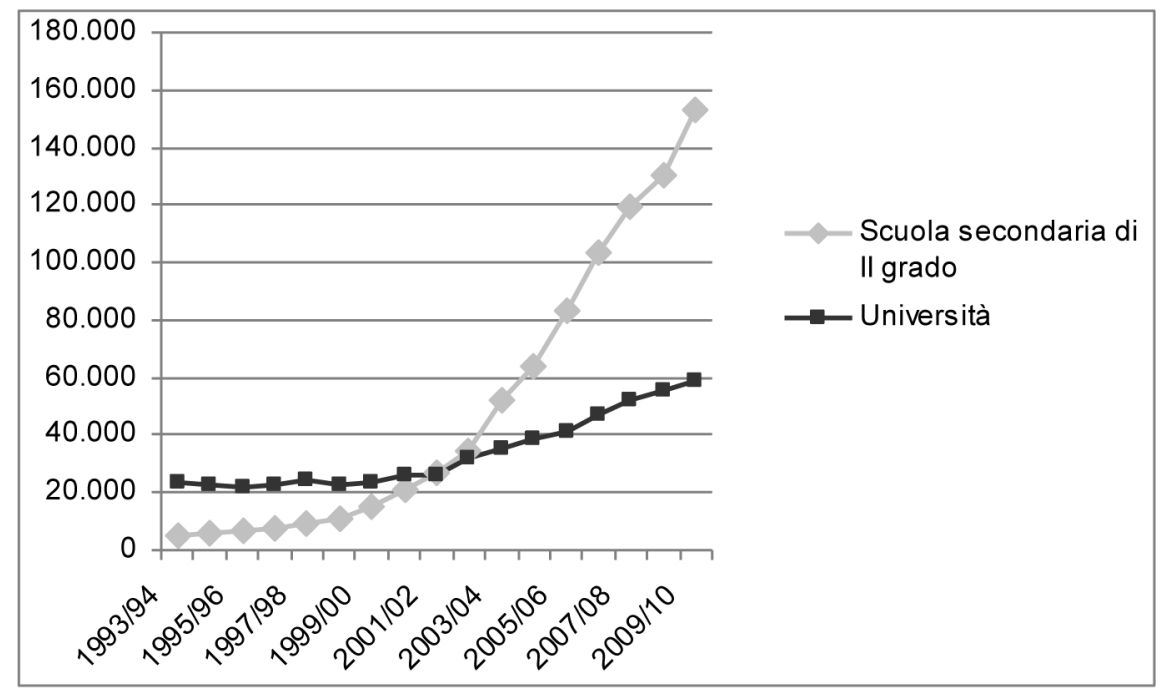

Fonte: Anagrafe studenti MIUR; MIUR, ISMU

Esiste - ci sembra - una sfida che non si è colta almeno in due direzioni: da un lato non si sono implementate al massimo quelle politiche di internazionalizzazione del sistema universitario, che (Erasmus a parte) non ha visto crescere significativamente il numero percentuale di studenti attratti in Italia esclusivamente per motivi di studio; dall'altro, si è attinto ancora poco da quell'universo migratorio che si è invece così profondamente riflesso nel sistema scolastico, ma che non ha trovato in esso quelle garanzie di equità di risultati tali da consentire una più ampia possibilità di ac- 
cesso nel sistema di istruzione universitaria. Tali considerazioni, così poste, aprono altre questioni ancora, che questa volta implicano anche le trasformazioni, di tipo qualitativo oltre che quantitativo, relative ai profili sociali degli studenti stessi, ricadendo su problemi di natura terminologica e di rilevazione statistica delle presenze. Se gli studenti di origine straniera che frequentavano l'università italiana negli anni '50, '60, '70, '80, possiamo ragionevolmente ritenerli migranti per motivi di studio, dunque soggetti che arrivano in Italia attratti dalla possibilità di frequentarvi l'università, come possiamo considerare gli studenti di altra cittadinanza che invece, nei decenni successivi, si sono iscritti all'università? Quanta parte hanno preso, in definitiva, i figli delle migrazioni, vale a dire quegli studenti che hanno ricevuto, in toto o in parte, una scolarizzazione in Italia, vi sono nati o vi sono arrivati al seguito delle loro famiglie? La questione non è di secondaria importanza e porta con sé numerosi problemi che rimandano a tipologie sociali che, anche al loro interno, possono articolarsi variamente e porre ulteriori questioni: lo studente che parte dal suo Paese per studiare in Italia da che cosa è attratto? Da quali Paesi proviene principalmente? Quali sono le sue condizioni socio-economiche di base? Quale lingua italiana parla? Dove vorrà spendere il suo titolo?

E ancora, lo studente che è cresciuto in Italia, di prima o di seconda generazione $^{3}$, in che cosa si differenzia? Come arriva all'università? Quali sono le comunità che meglio (o peggio) riescono ad avere accesso nel sistema universitario?

\subsection{L'universo composito degli studenti di cittadinanza non italiana: studenti internazionali e studenti stranieri scolarizzati in Italia}

La presenza di studenti di cittadinanza non italiana nel sistema universitario italiano e in quello post-secondario (se consideriamo i conservatori, le accademie delle belle arti, ecc.) è stata oggetto di pochi studi e ricerche, a fronte, probabilmente, di un fenomeno dalle dimensioni non troppo ampie e di una scarsa attenzione ad una questione che, in altri Paesi, ha avuto sviluppi ed esiti diversi. Sebbene i dati rilevati in Italia (così come in Francia, Grecia, Cina, ecc.) risentano di un sistema di classificazione diverso da quello utilizzato da altri Paesi, e ciò renda non sempre agevole la compara-

3. Nell'a.s. 2012/13 gli alunni con cittadinanza non italiana nati in Italia sono 371.332 e rappresentano il $47,2 \%$ sul totale degli alunni con cittadinanza non italiana (nel 2008/09 il $37 \%$ ). 
zione, risalta la grande differenza in ambito europeo ed extraeuropeo (sotto il 5\% l'Italia, con la Grecia, la Turchia, a fronte di Paesi come il Regno Unito, la Svizzera, l'Austria, il Belgio che superano la soglia del 15\% o della Francia, la Repubblica Ceca, il Belgio, che si collocano oltre il 10\%) (OECD, 2014).

La comparazione con altri contesti risente di una definizione che viene data di "studente all'estero", con una differenza non soltanto nominale ma anche sostanziale, che riguarda il motivo per cui ci si trova a studiare in un altro Paese. L'OECD (Organization for Economic Cooperation and Development) ravvisa tale limite e sottolinea come non sempre i dati siano facilmente comparabili, poiché variano i modi di intendere e classificare la presenza degli studenti all'interno dei diversi Paesi. In alcuni casi, come in Italia, sotto la definizione di studenti stranieri ricadono tutti gli studenti di cittadinanza non italiana, senza avere la possibilità di distinguere tra quei soggetti che sono residenti per effetto di precedenti migrazioni familiari e quelli che vi si recano per compiere gli studi universitari (studenti internazionali). Vale la pena riportare le definizioni dell'OECD (Ibidem, p. 352):

«Foreign students are those who are not citizens of the country in which the data are collected. While pragmatic and operational, this classification is inappropriate for capturing student mobility because of differing national policies regarding the naturalisation of immigrants. For instance, Australia has a greater propensity to grant permanent residence to its immigrant populations than Switzerland. This implies that even when the proportion of foreign students in tertiary enrolment is similar for both countries, the proportion of international students in tertiary education is smaller in Switzerland than in Australia. Therefore, for student mobility and bilateral comparisons, interpretations of data based on the concept of foreign students should be made with caution.

International or mobile students are those who left their country of origin and moved to another country for the purpose of study. Depending on countryspecific immigration legislation, mobility arrangements, such as the free mobility of individuals within the EU and the EEA, and data availability, international students may be defined as students who are not permanent or usual residents of their country of study or alternatively as students who obtained their prior education in a different country, including another EU country».

Anche in linea con precedenti ricerche (Lagomarsino, Ravecca, 2014), distingueremo (non senza difficoltà nella rappresentazione quantitativa del fenomeno) tra coloro che elettivamente decidono di venire a studiare nelle università italiane e coloro che, per precedenti scelte migratorie (proprie o della famiglia), trovandosi già a vivere in Italia, decidono di frequentarvi l'università. Se per molto tempo, la questione della presenza di studenti 
di altra cittadinanza poteva essere posta esclusivamente, o quasi, nei termini di attrattività del sistema universitario italiano, su basi legate a certe tradizioni culturali (si pensi alla musica, alle arti, o anche ad alcune discipline scientifiche) o su opportunità che in termini di diritto allo studio si sono gestite dentro accordi tra stati, se, ancora, le politiche di mobilità, Erasmus in primis, hanno in qualche modo reso più dinamici i flussi (in entrata e in uscita), oggi essa va presa in considerazione anche rispetto ad un fenomeno tanto nuovo quanto interessante per chi si occupa di intercultura ed inclusione: l'ingresso di quei soggetti che, per effetto delle migrazioni delle loro famiglie, si sono trovati a frequentare le scuole in Italia o, in alcuni casi, vi sono nati.

In questo senso la distinzione individuata dall'OECD (2014) tra lo studente internazionale (international student o mobile student) e lo studente straniero, può essere estremamente utile, in un contesto come quello attuale, che strutturalmente si presenta come multiculturale, se utilizzata per inquadrare in una prima e macroscopica tipologia sociale i soggetti iscritti nelle università.

Per questo motivo utilizzeremo, all'interno della nostra ricerca, dentro la categoria più ampia di studente di cittadinanza non italiana, la distinzione tra studente internazionale e studente straniero scolarizzato in Italia, a sottolineare una differenza sociologica, mai netta e non sempre schematica, tra i due macro-sottogruppi. Differenza, che si traduce in una serie di problemi pedagogici che affronteremo nel corso del volume.

Una lettura del fenomeno a partire dal tipo di permesso di soggiorno o dalla condizione giuridica legata alla cittadinanza, ci potrebbe dare la misura del fatto che lo studio in Italia rappresenta, per alcuni, motivo diretto e primario di "migrazione", per altri un motivo secondario, contestuale, riferito ad una vita in Italia che va letta e interpretata spesso dentro la cornice più ampia del progetto migratorio familiare. Se gli studenti internazionali si spostano dal loro Paese di origine con il proposito di studiare all'estero, gli studenti stranieri già residenti in Italia (d'ora in avanti studenti stranieri), pur non essendo ancora cittadini del Paese ospitante, sono residenti a medio-lungo termine, hanno frequentato la scuola, in toto o in parte, talvolta vi sono nati (le legislazioni in materia di cittadinanza rendono variabile questa condizione di stato in stato, a partire dall'applicazione del principio dello ius soli o di quello dello ius sanguinis). Ciò chiama in causa differenze sostanziali, che rimandano a fattori di tipo socio-culturale, socio-economico, non da ultima fattori di tipo linguistico e di conoscenza della cultura organizzativa e pedagogico-didattica del sistema formativo del Paese ospitante. In alcuni casi, si tratta di soggetti in attesa di acquisizione della cittadinanza, che nel nostro Paese, in applicazione del principio dello ius sanguinis risulta piuttosto lunga e complessa. 
Una ricerca dell'European Migration Network (EMN-Italia, 2012) disegna un quadro piuttosto interessante sulla situazione relativa agli studenti internazionali non comunitari presenti in Italia. A partire dai dati del Ministero degli Affari Esteri, è possibile avere un quadro più generale della situazione, che va però assunto con qualche cautela poiché esso si riferisce alla totalità dei permessi per motivi di studio, e, dunque, anche ai soggiorni brevi (corsi di lingua, ecc.), alle iscrizioni presso le Accademie delle Belle Arti, i Conservatori, le università straniere o religiose. La presenza degli studenti statunitensi si impone come la più consistente, anche se essi sono decisamente poco rappresentati nell'universo degli studenti iscritti nell'università italiana. Nel 2011 si registrano addirittura 15.162 permessi attivi, rilasciati per motivi di studio; di contro, nello stesso anno, risultano iscritti nelle università italiane soltanto 243 studenti $^{4}$. Si tratta dunque degli iscritti ai numerosi college nordamericani presenti in Italia e ad atenei pontifici, rispetto ai quali però non sono disponibili dati ufficiali. Si stima che siano circa 20.000 gli studenti statunitensi venuti nello stesso anno per compiere almeno un breve periodo di studio in Italia: tra questi, almeno 10.000 tra seminaristi e suore, iscritti nelle strutture pontificie e in possesso di titolo di soggiorno per motivi religiosi valido anche per la frequenza universitaria (IDOS, 2013). A seguire troviamo la Cina, per la quale sembra esistere un flusso consistente indipendente dalla comunità immigrata già residente in Italia (4.124 permessi per studio rilasciati nel 2011). Lo studio presso le università italiane può essere collegato ad alcune aree dalle cui si sono originati flussi di richiedenti asilo, come è il caso dell'Iran (1260 permessi rilasciati nel 2011) e del Medio Oriente. Anche alcuni Paesi africani, come il Camerun, attivano flussi significativi per motivi di studio.

Per analizzare più specificamente la situazione degli atenei, abbiamo proceduto alla consultazione della banca dati del MIUR (Anagrafe studenti), che meglio può aiutarci a definire il quadro di riferimento. Possiamo procedere ad una prima analisi della situazione italiana attraverso la Tabella 4 e il Grafico 3, che evidenziano come nel decennio considerato (2004-2014), il numero, assoluto e relativo, degli studenti di cittadinanza non italiana sia notevolmente aumentato, di fatto più che raddoppiato, passando da un'incidenza del 2,44\% nel 2004/05, al 4,17\% del 2013/14. Un trend in continuo aumento dunque, che rileva, sul macrodato, anche una maggiore presenza femminile, che si attesta, nell'ultimo anno preso in considerazione, al $58,9 \%$ sul totale della popolazione studentesca di altra cittadinanza.

4. Fonte: Anagrafe studenti MIUR (http://anagrafe.miur.it). 
Tab. 4 - Gli iscritti di cittadinanza non italiana all'università - Serie storica

\begin{tabular}{lccccc}
\hline A.A. & $\begin{array}{c}\text { Totale } \\
\text { iscritti }\end{array}$ & $\begin{array}{c}\text { Iscritti } \\
\text { cittadinanza } \\
\text { non italiana }\end{array}$ & $\begin{array}{c}\text { Incidenza } \\
\text { su iscritti }\end{array}$ & $\begin{array}{c}\% \\
\text { femmine }\end{array}$ & $\begin{array}{c}\text { Indice } \\
\text { di crescita } \\
\text { (base 100) }\end{array}$ \\
\hline $2004 / 05$ & 1.323 .802 & 33.054 & $2,44 \%$ & 58,33 & 100 \\
\hline $2005 / 06$ & 1.527 .000 & 38.949 & $2,55 \%$ & 59,04 & 117,8 \\
\hline $2006 / 07$ & 1.633 .789 & 44.560 & $2,73 \%$ & 59,42 & 134,8 \\
\hline $2007 / 08$ & 1.712 .173 & 50.443 & $2,95 \%$ & 59,37 & 152,6 \\
\hline $2008 / 09$ & 1.749 .929 & 55.576 & $3,18 \%$ & 59,21 & 168,1 \\
\hline $2009 / 10$ & 1.785 .320 & 60.176 & $3,37 \%$ & 58,78 & 182,0 \\
\hline $2010 / 11$ & 1.722 .346 & 63.845 & $3,57 \%$ & 58,98 & 193,1 \\
\hline $2011 / 12$ & 1.766 .205 & 66.797 & $3,78 \%$ & 58,81 & 202,0 \\
\hline $2012 / 13$ & 1.723 .852 & 68.947 & $4,00 \%$ & 58,85 & 208,6 \\
\hline $2013 / 14$ & 1.693 .804 & 70.635 & $4,17 \%$ & 58,90 & 213,7 \\
\hline
\end{tabular}

Fonte: Elaborazioni su database Anagrafe studenti MIUR

Graf. 3 - L'andamento della presenza degli studenti di cittadinanza non italiana (2004-2014)

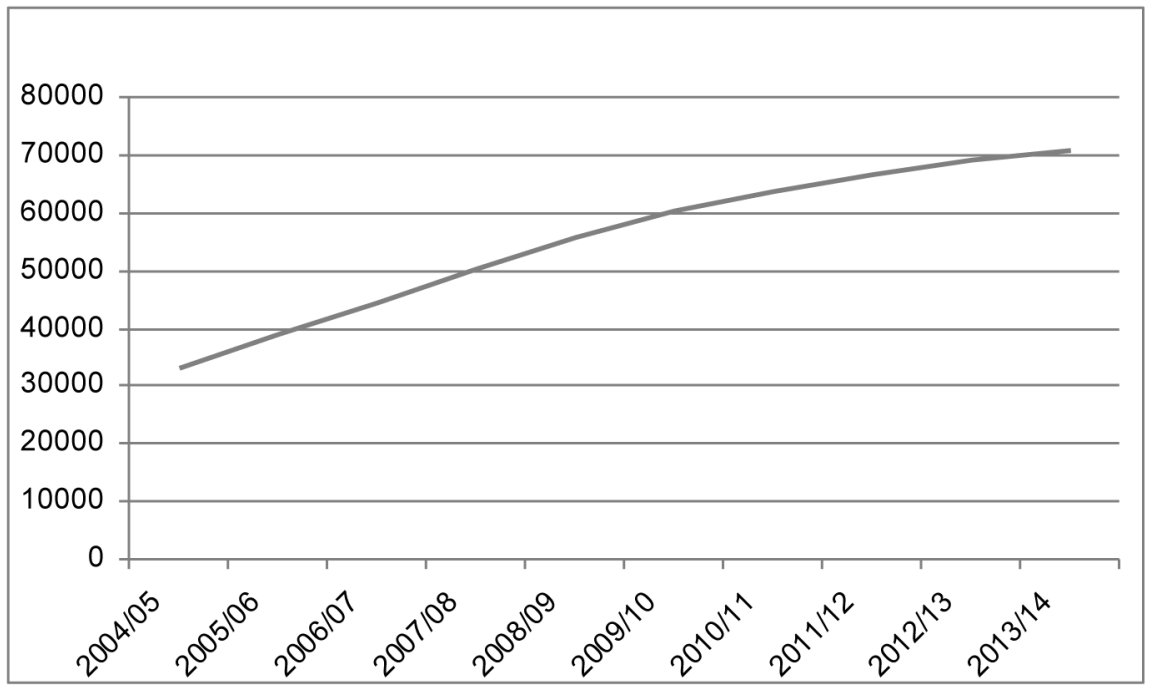

Fonte: Anagrafe studenti MIUR 
I dati non ci permettono tuttavia di riferire questo incremento alle diverse tendenze, che potrebbero essere meglio inquadrate a partire dalla tipologia del permesso di soggiorno, relative agli andamenti degli studenti internazionali e degli studenti stranieri scolarizzati in Italia. Possiamo formulare una prima idea di come siano cambiate le tendenze e per quanta parte, oggi, gli studenti stranieri scolarizzati in Italia, che dunque hanno studiato nelle scuole italiane e che sono inquadrati, a prescindere dalla loro presenza all'università, nel contesto multiculturale italiano, contribuiscono a rendere questo aumento così significativo.

Le Tabelle 5 e 6 ci forniscono una prima e indiretta indicazione a tal proposito, poiché ci permettono in alcuni casi, di formulare ipotesi partendo dalle prime 15 nazionalità presenti nei due anni accademici presi a riferimento.

L'Albania e la Romania si collocano, in modo stabile, con un tasso di incremento maggiormente visibile nel secondo caso, rispettivamente al primo e al terzo posto nella lista delle nazionalità. Nel contesto più generale dell'universo migratorio italiano, con riferimento al 2013, la Romania occupa la prima posizione tra le provenienze presenti, mentre l'Albania la terza. I marocchini, che nello scenario italiano, rappresentano la terza comunità presente, si collocano al nono posto dell'elenco 2013/14 (con una presenza, però, quasi triplicata nell'arco del decennio). Certamente, senza escludere che ci siano numerosi studenti che vengono a studiare in Italia senza avervi frequentato la scuola, attratti anche da parenti ed amici che vi si sono stabiliti precedentemente, possiamo ritenere che la presenza all'università di queste nazionalità almeno in parte sia il risultato dei più generali processi migratori.

Per le restanti nazionalità troviamo una differenza di posizioni in alcuni casi fortemente pronunciata: è il caso della Cina (seconda posizione del 2013/14 e diciannovesima nel 2004/05), così come della Germania (dalla quarta alla quindicesima posizione), della Grecia (dalla seconda all'ottava posizione), della Polonia e di altre nazionalità che escono (Francia, San Marino, Bulgaria, Croazia, Svizzera) o entrano nella lista delle prime quindici (Moldavia, Ucraina, Russia, Ecuador). 
Tab. 5 - Le prime 15 nazionalità - Confronto iscritti università 2004/05 e 2013/14

\begin{tabular}{lrr|lrr}
\hline \multicolumn{2}{c|}{ Le prime 15 nazionalità a.a. $2004 / 05$} & Le prime 15 nazionalità a.a. $2013 / 14$ \\
\hline Albania & 8.898 & $26,92 \%$ & Albania & 11.036 & $15,62 \%$ \\
Grecia & 3.025 & $9,15 \%$ & Cina & 7.176 & $10,16 \%$ \\
Romania & 1.351 & $4,09 \%$ & Romania & 6.854 & $9,70 \%$ \\
Germania & 1.302 & $3,94 \%$ & Iran & 2.862 & $4,05 \%$ \\
Camerun & 1.195 & $3,62 \%$ & Camerun & 2.752 & $3,90 \%$ \\
Polonia & 980 & $2,96 \%$ & Moldavia & 2.121 & $3,00 \%$ \\
Croazia & 895 & $2,71 \%$ & Perù & 2.073 & $2,93 \%$ \\
Israele & 842 & $2,55 \%$ & Grecia & 2.046 & $2,90 \%$ \\
Svizzera & 829 & $2,51 \%$ & Marocco & 1.956 & $2,77 \%$ \\
Perù & 785 & $2,37 \%$ & Ucraina & 1.896 & $2,68 \%$ \\
Marocco & 697 & $2,11 \%$ & Russia & 1.621 & $2,29 \%$ \\
Francia & 626 & $1,89 \%$ & Israele & 1.343 & $1,90 \%$ \\
Iran & 584 & $1,77 \%$ & Polonia & 1.194 & $1,69 \%$ \\
San Marino & 576 & $1,74 \%$ & Ecuador & 1.147 & $1,62 \%$ \\
Bulgaria & 540 & $1,63 \%$ & Germania & 1.114 & $1,58 \%$ \\
\hline
\end{tabular}

Tab. 6 - Le prime 15 nazionalità - Confronto con popolazione residente in Italia al $1^{\circ}$ gennaio 2013

\begin{tabular}{l|r|r|r|r}
\hline \multicolumn{2}{c|}{$\begin{array}{c}\text { Le prime 15 nazionalità - } \\
\text { Iscritti a.a. 2013/14 } \\
\text { (dati MIUR) }\end{array}$} & $\begin{array}{c}\text { Le prime 15 nazionalità in } \\
\text { Italia al } 1^{\circ} \text { gennaio 2013 } \\
\text { (dati ISTAT) }\end{array}$ & Incidenza\% \\
\hline 1. Albania & 11.036 & $2^{\circ}$ & 495.709 & 2,22 \\
2. Cina & 7.176 & $4^{\circ}$ & 256.846 & 2,79 \\
3. Romania & 6.854 & $1^{\circ}$ & 1.081 .400 & 0,63 \\
4. Iran & $\mathbf{2 . 8 6 2}$ & $\mathbf{4 5}^{\circ}$ & $\mathbf{8 . 9 9 5}$ & $\mathbf{3 1 , 8 1}$ \\
5. Camerun & $\mathbf{2 . 7 5 2}$ & $\mathbf{4 3}^{\circ}$ & $\mathbf{1 1 . 8 8 0}$ & $\mathbf{2 3 , 1 6}$ \\
6. Moldavia & 2.121 & $7^{\circ}$ & 149.434 & 1,41 \\
7. Perù & 2.073 & $10^{\circ}$ & 109.851 & 1,88 \\
8. Grecia & $\mathbf{2 . 0 4 6}$ & $\mathbf{5 6}$ & $\mathbf{7 0 . 8 1}$ & $\mathbf{2 8 , 8 9}$ \\
9. Marocco & 1.956 & $3^{\circ}$ & 454.773 & 0,43 \\
10. Ucraina & 1.896 & $5^{\circ}$ & 219.050 & 0,86 \\
11. Russia & $\mathbf{1 . 6 2 1}$ & $\mathbf{2 6 ^ { \circ }}$ & $\mathbf{3 4 . 4 8 3}$ & $\mathbf{4 , 7 0}$ \\
12. Israele & $\mathbf{1 . 3 4 3}$ & $\mathbf{8 5}$ & $\mathbf{2 . 1 0 5}$ & $\mathbf{6 3 , 8 0}$ \\
13. Polonia & 1.194 & $11^{\circ}$ & 97.566 & 1,22 \\
14. Ecuador & 1.147 & $15^{\circ}$ & 91.861 & 1,24 \\
15. Germania & 1.114 & 260 & 38.136 & 2,92 \\
\hline Popolazione residente complessivamente in Italia: 60.782 .668 & 2,78 \\
Iscritti nelle università italiane: 1.693.804 & & \\
\hline
\end{tabular}

Le tabelle appena presentate ci consentono di approfondire ulteriormente e di individuare, tra gli studenti di diversa nazionalità, quelli che più contribuiscono ad essere rappresentativi della tipologia di studenti inter- 
nazionali. Considerando come parametri di riferimento l'incidenza percentuale del numero degli iscritti all'università sul totale della popolazione italiana (pari al 2,78\%), nonché il confronto sulle posizioni occupate tra le presenze all'università e le presenze nel più generale contesto italiano, abbiamo osservato che alcune comunità spiccano per essere maggiormente implicate in un flusso migratorio per motivi di studio: è il caso dell'Iran (al quarto posto tra le nazionalità presenti nel sistema universitario e addirittura al $45^{\circ}$ posto tra le comunità residenti in Italia, con un'incidenza degli studenti sul totale della popolazione iraniana nel territorio nazionale del 31,81\%); è il caso del Camerun, che mantiene nei due anni accademici di riferimento la stessa posizione (Tab. 5), a fronte però di una posizione nell'elenco delle nazionalità presenti in Italia decisamente in basso (al $43^{\circ}$ posto, incidenza del $23,16 \%$ ); è il caso della Grecia, che verrà meglio analizzato nel capitolo 7; è il caso degli studenti di cittadinanza israeliana, molto spesso di etnia palestinese (12 ${ }^{\mathrm{a}}$ posizione, a fronte dell' $85^{\mathrm{a}}$, con un'incidenza percentuale addirittura del 63,80\%). Interessante il caso degli studenti cinesi, che si attestano nel 2013-2014 come la seconda nazionalità presente. A distanza di 10 anni (nel 2004/05 occupavano la ventesima posizione, con 385 iscritti, incidenti per l'1,16\% sull'intera popolazione cinese presente in Italia), troviamo una crescita di quasi 20 volte, con 7.176 iscritti. Nonostante quella cinese sia considerata, in ambito internazionale, come una model minority, o super minority (Li e Wang, 2008) in quanto ricorrente in posizioni apicali nei sistemi formativi rispetto alle altre minoranze immigrate, dobbiamo far presente che gli iscritti cinesi rappresentano un caso "ambiguo" rispetto alla loro collocazione tra gli studenti internazionali e gli studenti stranieri scolarizzati in Italia. L'incremento va spiegato infatti anche a partire dalle recenti politiche bilaterali tra Italia e Cina, che hanno dato corpo al Progetto "Marco Polo" (e al Progetto "Turandot" per l'alta formazione artistica e musicale), che prevede un semestre di studio dell'italiano nelle università per stranieri ed il successivo ingresso ai vari corsi di laurea. In questo caso rimane difficile scorporare il dato rispetto a quegli studenti, figli di migranti, che hanno studiato in Italia e gli studenti internazionali. Nell'a.a. 2012/13, da fonti del Ministero degli Esteri e Uni-Italia 5 , il Programma "Marco Polo" ha attirato quasi 4.000 studenti, soprattutto dai distretti consolari di Pechino, Shangai e Canton, (su un totale di iscritti 6.901 studenti cinesi iscritti), il che ci porta a relativizzare l'eventuale ruolo di super minority delle comunità cinesi già residenti in Italia.

5. Ministero degli Affari Esteri e della Cooperazione Internazionale, Italia-Cina: crescono iscrizioni studenti cinesi in università italiane, comunicazione del 21/02/2012 (www.esteri.it/mae/it/sala_stampa/archivionotizie/approfondimenti/2012/02/20120221_italiacina.html). 
Se ragioniamo sulla comparazione tra le prime presenze nell'università e nella scuola, riusciamo da un lato a confermare l'individuazione di quelle nazionalità che in maniera più cospicua possiamo fare rientrare nella categoria degli studenti internazionali (Grecia, Israele, Polonia, Germania), dall'altro ad individuare le comunità immigrate che più debolmente passano dalla scuola all'università (India, Pakistan, Tunisia, Egitto, Macedonia, Bangladesh, Romania).

Con la Tabella 7 possiamo comparare la presenza straniera nella scuola secondaria di II grado e all'università. La differenza percentuale tra gli iscritti nella scuola e all'università va considerata come dato da leggere alla luce delle considerazioni fino ad ora fatte: se nei passaggi da un ordine di scuola all'altra si ragiona coerentemente su un universo sociologico di riferimento generale (le comunità migranti), per l'università si pone il problema di considerare gli studenti internazionali e, dunque, di verificare effettivamente quanta parte, sul $4 \%$ rilevato, prendano gli studenti stranieri scolarizzati in Italia.

Tab. 7 - Iscritti di cittadinanza non italiana nella scuola secondaria di II grado e all'università - 2012/13

\begin{tabular}{|c|c|c|c|c|c|}
\hline \multicolumn{3}{|c|}{$\begin{array}{c}\text { Scuola secondaria } \\
\text { di Il grado } 6,6 \% \text { su totale } \\
\text { popolazione studentesca }\end{array}$} & \multicolumn{3}{|c|}{$\begin{array}{c}\text { Iscritti università } \\
4,0 \% \text { su totale } \\
\text { popolazione studentesca }\end{array}$} \\
\hline 30.478 & $17,4 \%$ & 1. Romania & Albania & 11.300 & $16,39 \%$ \\
\hline 23.614 & $13,5 \%$ & Albania & Cina & & $10,01 \%$ \\
\hline 15.253 & $8,7 \%$ & Marocco & Romania & 6.362 & $9,23 \%$ \\
\hline 8.649 & $4,9 \%$ & Moldova & Camerun & 2.784 & $4,04 \%$ \\
\hline 7.996 & $4,6 \%$ & 5. Ucraina & Iran & 2.697 & $3,91 \%$ \\
\hline 6.348 & $3,6 \%$ & 6. Cina & Grecia & 2.221 & $3,22 \%$ \\
\hline 6.269 & $3,6 \%$ & Ecuador & Perù & 2.018 & $2,93 \%$ \\
\hline 6.239 & $3,6 \%$ & 8. Perù & Moldova & 1.976 & $2,87 \%$ \\
\hline 5.454 & $3,1 \%$ & 9. Filippine & 9. Marocco & 1.874 & $2,72 \%$ \\
\hline 4.402 & 2,59 & 10. India* & 10. Ucraina & 1.758 & $2,55 \%$ \\
\hline 3.177 & $1,8 \%$ & 11. Macedonia & 11. Russia & 1.502 & $2,18 \%$ \\
\hline 2.943 & 1,7 & 12. Tunisia* & 12. Israele & 1.467 & $2,13 \%$ \\
\hline 3.056 & 1,70 & 13. Pakistan & 13. Polonia & 1.2 & $1,74 \%$ \\
\hline 2.303 & $1,3 \%$ & 14. Egitto* & 14. Germania & 1.129 & $1,64 \%$ \\
\hline 1.882 & $1,1 \%$ & 15. Bangladesh & 15. Ecuador & 1.031 & $1,50 \%$ \\
\hline 47.057 & $26,9 \%$ & 16. Altre & 16. Altre & 22.726 & $32,96 \%$ \\
\hline
\end{tabular}

* posizioni su elenco nazionalità presenti all'università

India: $18^{\circ}$ posto, 836 studenti - Pakistan: $20^{\circ}$ posto, 784 studenti - Tunisia: $21^{\circ}$ posto, 771 studenti - Egitto: $28^{\circ}$ posto, 664 studenti - Macedonia: $30^{\circ}$ posto, 519 studenti - Bangladesh, $31^{\circ}, 451$ studenti - Filippine: $33^{\circ}$ posto, 445 studenti

Fonti: dati MIUR e ISMU, 2014; Anagrafe studenti MIUR 
Anche in questo caso, se consideriamo le prime 15 comunità presenti nella scuola, verifichiamo la condizione di "debolezza" delle stesse all'interno dell'università, come nel caso della Macedonia (undicesima posizione nella scuola, trentesima all'università), del Bangladesh, delle Filippine, dell'India, dell'Egitto, della Tunisia, del Pakistan. Di contro, si osserva come alcune comunità presenti all'università (greca, israelo-palestinese, polacca, tedesca) non sono presenti nella lista riferita alla scuola secondaria e ciò ci lascia confermare la loro specificità di studenti internazionali.

\subsection{Avanguardie migranti: i figli delle migrazioni all'univer- sità}

I dati sulle immatricolazioni nell'ultimo decennio registrano un trend negativo sui valori riferiti al numero complessivo degli studenti (336.397 nel 2004 - 269.450 nel 2014). Se consideriamo invece soltanto la componente degli studenti di cittadinanza non italiana, osserviamo un trend positivo, che ha visto salire il loro numero da 9.797 nel 2004 a 13.069 nel 2014 (Tab. 8). Il Grafico 4 riporta gli andamenti sui valori indicizzati (base 100) e mostra molto chiaramente come ci troviamo di fronte ad un andamento che richiama anche quello in atto ormai da anni nella scuola e nella composizione demografica della società nel suo complesso. Ciò significa che la presenza straniera, legata ai più generali movimenti migratori, sta diventando un carattere strutturale dell'università italiana, che vede (e vedrà sempre di più) compiersi un'accelerazione della sua trasformazione, qualitativa e quantitativa insieme, in direzione multiculturale. A ciò si aggiungano anche quegli studenti che non vengono presi in considerazione dalle statistiche poiché hanno acquisito la cittadinanza italiana, ma per i quali va

Tab. 8 - Confronti tra immatricolati (lauree triennali e a ciclo unico), immatricolati di altra cittadinanza, con diploma estero o italiano

\begin{tabular}{lcccccc}
\hline & $\begin{array}{c}\text { Immatri- } \\
\text { colati }\end{array}$ & $\begin{array}{c}\text { Di cui di } \\
\text { cittad. } \\
\text { non } \\
\text { italiana }\end{array}$ & $\begin{array}{c}\text { \% cittadi- } \\
\text { nanza } \\
\text { non } \\
\text { italiana }\end{array}$ & $\begin{array}{c}\text { Con } \\
\text { diploma } \\
\text { estero }\end{array}$ & $\begin{array}{c}\text { \% diploma } \\
\text { estero } \\
\text { (stima } \\
\text { studenti } \\
\text { interna- } \\
\text { zionali) }\end{array}$ & $\begin{array}{c}\text { Stima } \\
\text { studenti } \\
\text { stranieri* } \\
\text { (diploma } \\
\text { italiano) }\end{array}$ \\
\hline $2004 / 05$ & 336.397 & 9.797 & $2,91 \%$ & 8.439 & $2,51 \%$ & $0,4 \%$ \\
$2008 / 09$ & 293.875 & 12.298 & $4,18 \%$ & 8.595 & $2,92 \%$ & $1,26 \%$ \\
$2013 / 14$ & 269.450 & 13.069 & $4,85 \%$ & 6.016 & $2,23 \%$ & $2,62 \%$ \\
\hline
\end{tabular}

Fonte: Dati Anagrafe studenti MIUR 
considerata una storia migratoria familiare che può in qualche modo avvicinarli alla componente degli studenti stranieri.

Graf. 4 - Trend immatricolati e immatricolati di cittadinanza non italiana (indice base 100)

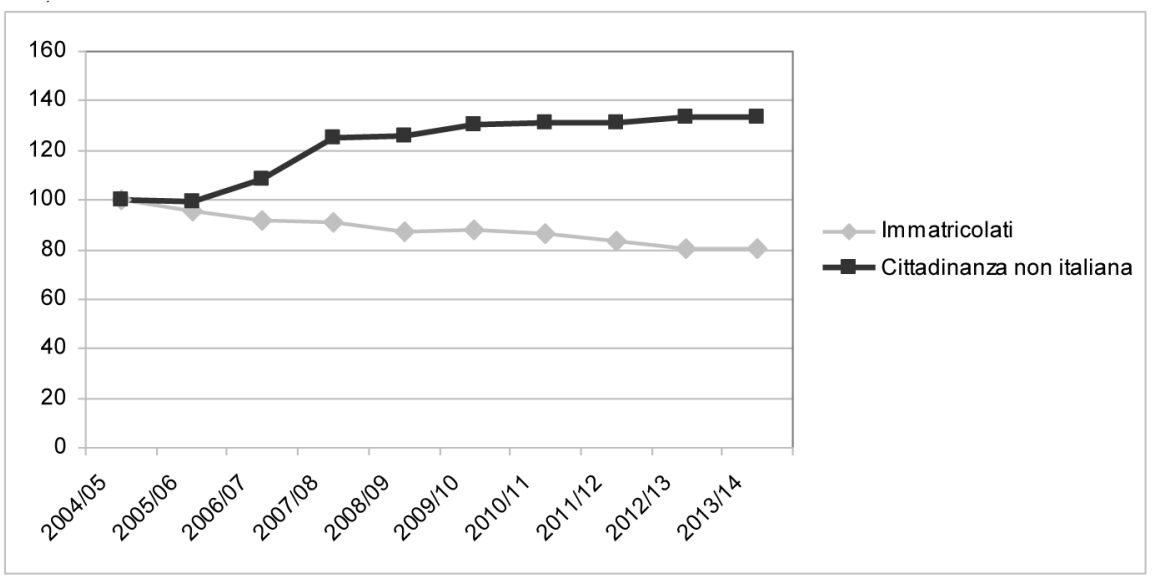

Graf. 5 - Immatricolati per tipologia di diploma (italiano o estero)

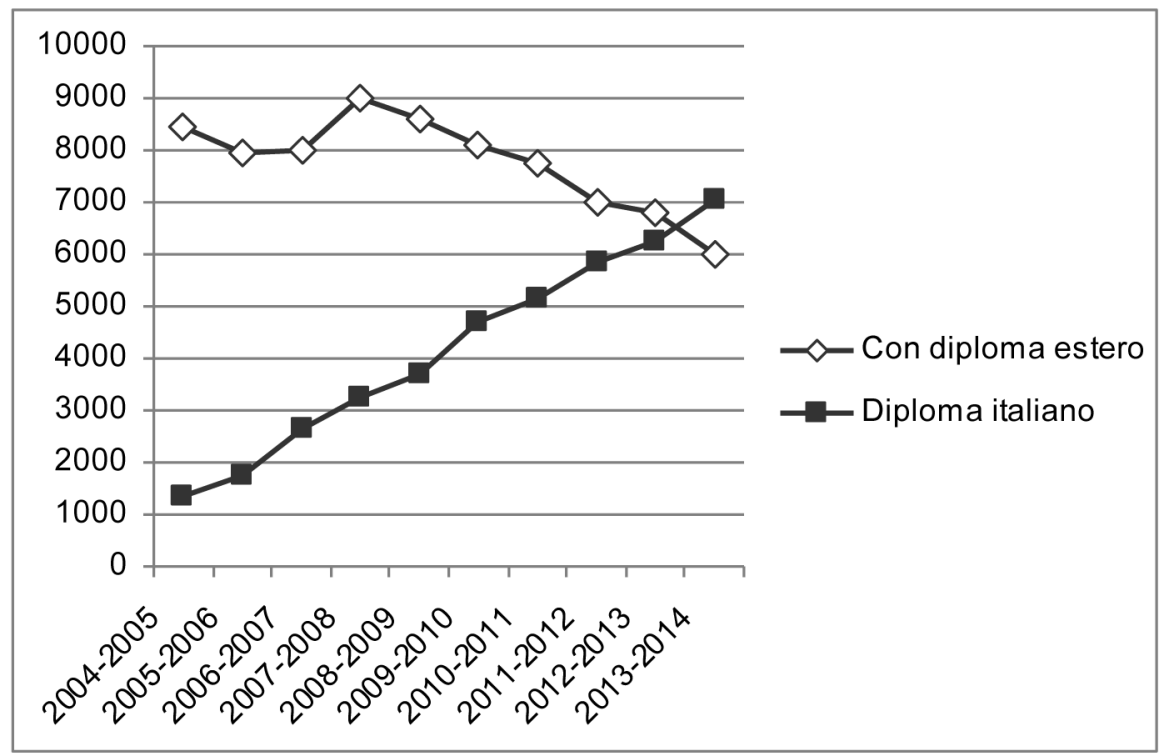


I dati sugli immatricolati ci consentono anche di avere una stima orientativa sul numero degli studenti internazionali e degli studenti stranieri scolarizzati in Italia, a partire dal possesso del titolo di studio utile ai fini dell'iscrizione all'università (Graf. 5). L'a.a. 2012/13 vede tra i 216.497 immatricolati ai corsi di laurea triennale, 11.796 studenti $(5,4 \%)$ di cittadinanza diversa da quella italiana. Di questi, 6.691 hanno conseguito il diploma di scuola secondaria in Italia, rappresentando il 56,7\% degli studenti di cittadinanza non italiana e il 3,1\% degli immatricolati in totale (Colombo e Ongini, 2014).

Prima di continuare con l'analisi, avanziamo alcune cautele: il titolo estero non sempre corrisponde necessariamente alla posizione dello studente internazionale, poiché non possiamo ritenere infrequente la possibilità che ad iscriversi siano persone, talvolta adulte, già presenti in Italia per motivi diversi o richiamate dalle famiglie solo dopo il conseguimento del titolo di studio superiore. Al contrario, gli studenti che hanno acquisito un titolo di scuola secondaria di II grado in Italia sono sostanzialmente inquadrabili all'interno di quella componente che abbiamo definito come studenti stranieri, vale a dire quei soggetti che, per vari motivi, soprattutto legati a progetti migratori diversi dalle motivazioni di studio, si trovano a risiedere in Italia già dall'età scolare. Abbiamo osservato, nella tabella e nei grafici, come a fronte di un trend che vede una relativa decrescita degli studenti con diploma estero nel decennio considerato, gli studenti con diploma italiano siano rapidamente aumentati, con un peso percentuale dello $0,4 \%$ nel 2004/05 e un peso del 2,62\% nel 2013/14. Se nel 2004/05 gli studenti stranieri che hanno acquisito un titolo di accesso italiano erano il 13,9\% sul totale degli studenti di cittadinanza non italiana, nel 2013/14 arriviamo al 53,9\%, un cambiamento, che solo nell'arco di un decennio, stravolge l'universo sociologico di partenza. Nella ricerca presentata nella seconda parte del volume, che ha ristretto il suo campo di indagine all'Università degli Studi dell'Aquila, il campione raggiunto (iscritti 2013) si compone per il $38,2 \%$ di studenti stranieri, nati in Italia $(1,7 \%)$ o che vi hanno frequentato la scuola (36,5\%), e per il 61,8\% di studenti internazionali.

Il cambiamento appena richiamato si presenta come sostanziale almeno in due direzioni, che hanno forti implicazioni in tema di politiche formative accademiche:

- l'attrattività del sistema universitario italiano per gli studenti internazionali non ha fatto, se si considerano i dati percentuali, sostanziali passi avanti rispetto ai decenni che ci precedono;

- l'aumento del numero degli studenti stranieri scolarizzati in Italia pone sfide che possono essere affrontate soltanto nell'ottica della continuità formativa scuola-università e nell'ottica di quella pedagogia interculturale che molto si è spesa negli ultimi decenni sul fronte della scolarizzazione dei migranti e che oggi deve misurarsi anche con i loro percorsi universitari. 


\subsection{II sistema a imbuto: i passaggi dalla scuola all'univer- sità}

La sfida che l'università si pone dipende in larga misura anche dalle sfide che il sistema scolastico italiano si trova non senza fatica ad affrontare, con risposte che ancora non consentono al sistema stesso di potersi definire pienamente equo rispetto agli esiti, e dunque rispetto alla qualità della formazione. Per quanto nel corso degli anni siano state messe a punto strategie interessanti in tema di accoglienza e integrazione (MIUR, 2014), i dislivelli nei tassi di non ammissione tra stranieri e italiani nei vari ordini e gradi risultano ancora troppo ampi: prendendo a riferimento l'a.s. 2012/13, nella scuola primaria a fronte dello $0,3 \%$ di non ammessi alla classe successiva di nazionalità italiana, troviamo un $2,1 \%$ di studenti di cittadinanza diversa; nella secondaria di I grado, troviamo rispettivamente un 3,0\% a fronte del 9,1\%; nella secondaria, che è il segmento che maggiormente ci riguarda nell'ottica di una continuità scuola-università, il 12,7\% di non ammessi italiani viene contrassegnato dal $27,0 \%$ di studenti stranieri, con una differenza percentuale di 14,3 (Colombo e Ongini, 2014). Rispetto ai risultati dell'esame di stato, si diploma il 98,3\% degli stranieri a fronte del 99,3\% degli italiani ammessi, con uno svantaggio relativo dei primi, anche se piuttosto contenuto $(-1,0 \%)$. Tale svantaggio è più evidente negli istituti tecnici e professionali rispetto ai licei e agli istituti di vocazione artistica (Ibidem).

Nonostante i percorsi scolastici siano segnati da ostacoli e difficoltà maggiori, e a diplomarsi arrivino i soggetti più determinati, più performanti o quelli che hanno incontrato migliori opportunità nella scuola, anche per quanto riguarda i passaggi dalla scuola secondaria di II grado all'università, si osserva una differenza piuttosto significativa tra i diplomati italiani e quelli di altra cittadinanza: tra i diplomati del 2012 si sono iscritti all'università il 50,8\% degli studenti italiani e il 35,3\% degli studenti di altra cittadinanza, con una differenza percentuale del 15,5\% (MIUR, 2013a). Rispetto alle scelte, le aree disciplinari preferite dagli studenti non differiscono molto tra italiani e stranieri, tranne che per una maggiore concentrazione nelle aree economico-statistica (21\% degli stranieri contro il 14,6\% degli immatricolati totali), ingegneristica (16\% contro il 15\%) e linguistica (13\% contro il 7\%). Per quanto riguarda i Paesi di provenienza, si notano invece differenze significative nelle scelte: il $43 \%$ degli studenti cinesi privilegia l'area economico-statistica, il 32\% degli studenti indiani l'area ingegneristica, il 28\% degli ucraini l'area linguistica.

Ci troviamo di fronte ad un sistema ad "imbuto" sul quale è necessario riflettere adeguatamente per individuare scelte di sistema che possano col- 
mare il gap all'interno di una situazione generale anch'essa piuttosto complessa e problematica. Le politiche universitarie, in questo senso, non solo devono essere ancora messe a punto, ma potranno essere implementate solo a fronte di politiche formative scolastiche in grado di costruire profili in ingresso quantitativamente e qualitativamente più solidi, dunque attraverso azioni che producano, nell'ottica dell'equità, maggiori flussi di accesso e migliori forme di successo.

\subsection{I tempi sono maturi: intercultura all'università}

Sono poche le ricerche sugli studenti di cittadinanza non italiana all'università. Significativo lo studio, di approccio sociologico, svolto nell'ambito dell'Università di Genova che ha centrato l'attenzione, attraverso una metodologia qualitativa, proprio sugli studenti scolarizzati in Italia, i figli delle migrazioni, tra i quali non poco peso oggi hanno le seconde generazioni. La ricerca ha messo in luce come, a fronte di un'idea comunemente diffusa secondo cui chi si iscrive all'università abbia già superato gli ostacoli che rendono difficile il successo formativo e l'integrazione culturale, linguistica e sociale, le cose non sono così scontate e, nelle tipologie individuate dai ricercatori, continuano spesso ad essere attivi quei fattori di influenza negativi che rendono i percorsi non sempre lineari e liberi da condizionamenti legati alla posizione sociale degli studenti. Se per alcuni, i diritti alla meta, si osserva una condizione di partenza caratterizzata da famiglie con buoni livelli culturali, un inserimento nella scuola italiana generalmente di lunga durata, per altri i percorsi sono più articolati e complessi: i camminatori inesperti - in posizione intermedia - vivono l'aspirazione dell'emancipazione socio-economica che un titolo superiore potrebbe promuovere come desiderio di riscatto sociale, ma con un capitale culturale, sociale e linguistico segnato dallo svantaggio della condizione familiare e da percorsi pregressi talvolta deboli e non lineari; nel caso dei disorientati, le limitate risorse economiche, un background culturale ed economico familiare generalmente basso, la necessità di lavorare per aiutarsi, costituiscono un fattore di svantaggio che li rende la categoria più debole e, dunque, diremmo a maggiore rischio di dispersione. In questo ultimo caso le scelte amicali e di socializzazione sono spesso orientate esclusivamente verso i connazionali. Un ulteriore dato significativo, dal punto di vista pedagogico, che la ricerca offre, riguarda il ruolo che ha avuto la scuola: anche nel caso dei disorientati, infatti, l'aver frequentato scuole con buona sensibilità interculturale e con adeguata cultura organizzativa e didattica è un fattore ricorrente. 
La ricerca presentata in questo volume ha come campo di indagine l'Università dell'Aquila e si è mossa essenzialmente all'interno di una metodologia di tipo quantitativo. Affidando ai prossimi capitoli il compito di chiarire i diversi risultati nel dettaglio, si vuole anticipatamente fornire qualche spunto di riflessione che ci consente di mettere meglio a fuoco la situazione degli studenti con cittadinanza non italiana all'università e di sottolineare come, se nella scuola ormai il dibattito pedagogico-interculturale sta entrando all'interno della sua cultura organizzativa e didattica, ormai i tempi sono più che maturi anche per il mondo accademico.

La ricerca ha evidenziato non soltanto la presenza, non più sottile, di studenti figli delle migrazioni, ma anche - più generalmente considerando tutti gli studenti di cittadinanza non italiana - la presenza nel contesto di fattori che incidono negativamente sulla loro riuscita accademica e sul loro inserimento socio-culturale. A prescindere dalle differenze di background (status socio-economico e culturale delle famiglie, percorsi scolastici, provenienze), che pure rappresentano elementi particolarmente interessanti ed utili per un ripensamento del sistema, interrogando i soggetti sulla quotidianità delle loro relazioni, sui dispositivi didattici e organizzativi, ci si rende conto del fatto che non di minore importanza sono quei fattori che scandiscono e regolano la vita accademica, sia nelle pieghe informali dei rapporti tra attori, sia nei momenti più formali e istituzionali. Presenti, in molti casi, il pregiudizio e forme di discriminazione, presenti alcuni dispositivi didattici non in grado di neutralizzare, almeno all'inizio delle carriere, una non piena conoscenza linguistica in italiano, assente l'attenzione verso la qualità della vita sociale di quei soggetti (in questo caso ciò vale soprattutto per gli studenti internazionali) per i quali l'arrivo è spesso segnato da un senso di solitudine e difficoltà di integrazione. Il progetto "Unidiversità", nel quale la ricerca si inserisce, segnala un'attenzione dell'istituzione (e degli altri partner) e l'intenzione di agire attraverso azioni di breve e media durata.

Se questa sensibilità si sta facendo avanti a livello di singoli atenei, va rilevato che sarebbe necessaria una visione di insieme del problema, una visione che riguardi l'intero sistema universitario e lo orienti, anche nelle scelte locali, a partire da principi e prassi che possano rendere lo studio universitario maggiormente attrattivo, accessibile e libero da influenze che condizionamenti di tipo socio-economico e di tipo socio-culturale possono esercitare: non si tratta soltanto di richiamare il principio dell'uguaglianza delle opportunità, e quindi il diritto allo studio e delle varie forme di sostegno economico, ma anche quei fattori di processo che rendono effettivamente equo, sul piano sostanziale, l'intero sistema: dunque, un orientamento mirato, un supporto maggiore in lingua italiana e nell'italiano declinato nelle varie microlingue disciplinari, una maggiore accessibilità ai contenu- 
ti di studio attraverso il ricorso all'inglese, una valutazione dei profitti che tenga conto delle competenze linguistiche (almeno transitoriamente), un'attenzione alle relazioni interculturali (spesso segnate da percezioni alterate), un'attenzione al tempo libero. Sarebbe opportuno dunque agire tanto sulla rimozione di ostacoli di varia natura quanto sulla messa a punto di prassi positive; coerentemente con quanto Favaro ha messo in luce in riferimento al tema della mediazione culturale (Favaro e Fumagalli, 2004), diventa necessario intervenire almeno su tre livelli: uno pratico-orientativo (accoglienza, orientamento, accesso ai servizi), uno linguistico-comunicativo, uno psico-sociale. Peraltro, il contrasto alla dispersione - che sembra essere uno dei temi maggiormente sottolineati nell'agenda del sistema universitario - non può non tener conto di questo e del fatto che sempre di più gli studenti di altra cittadinanza (e tra questi quelli figli delle migrazioni) popoleranno le aule accademiche.

Bisognerebbe riflettere sulla reattività che la scuola ha avuto nel definire un quadro orientativo in tema di alunni stranieri: in solo pochi decenni di storia dell'inserimento scolastico, seppure con un'implementazione a macchia di leopardo e non sempre lineare (Vaccarelli, 2011), il Ministero ha definito quegli orientamenti, in accordo con le più rilevanti acquisizioni in campo pedagogico-interculturale, che hanno consentito di disegnare un modello di riferimento per le singole istituzioni scolastiche: con una normativa che ha segnato diversi passaggi, oggi la scuola dispone di un recente documento le "Linee guida per l'accoglienza e l'integrazione" (MIUR, 2014), che fa da quadro di riferimento per tutti quegli aspetti organizzativi e amministrativi (iscrizioni in primis) e per le prassi educative da mettere in campo.

L'università è il luogo in cui la pedagogia interculturale, con il suo statuto teorico ed epistemologico, ha preso forma e peso e dove, oggi, diventa necessario rivolgere l'attenzione; ciò, attraverso la ricerca e una progettualità che trasferisca e contestualizzi i saperi ormai maturi che la stessa pedagogia interculturale ha costruito nel confronto con la scuola. E i tempi, lo ripetiamo, si sono fatti maturi per pensare, così come ha fatto la scuola, non soltanto il presente, ma anche il futuro. 

Parte II

Studenti italiani e studenti di altra cittadinanza allo specchio: la ricerca e l'analisi dei dati 



\title{
6. Una ricerca sugli studenti dell'Università dell'Aquila: studenti stranieri, studenti internazionali, studenti italiani
}

\author{
di Alessandro Vaccarelli
}

\subsection{Obiettivi, strumenti e metodologie}

La ricerca qui presentata - inserita nel più ampio progetto "Unidiversità" - è stata condotta dal mese di ottobre 2014 al mese di marzo 2015. Il coordinatore della ricerca si è avvalso della collaborazione di 10 operatori di banca dati, con funzione anche di somministratori, e dell'Ufficio statistico dell'Università dell'Aquila'. L'obiettivo generale del progetto prevedeva la creazione di una banca dati sulle condizioni di vita e di studio degli studenti stranieri. All'interno di tale obiettivo si sono definiti obiettivi specifici che possiamo sommariamente sintetizzare: 1) esplorare le situazioni di partenza degli studenti attraverso dati relativi alla provenienza geografica, alle condizioni socio-economiche, alla scolarizzazione pregressa, alle competenze linguistiche; 2) studiare i percorsi accademici rispetto alle loro tipologie, ai risultati, ai fattori di successo o insuccesso, alla motivazione allo studio; 3) esplorare le dimensioni della vita sociale, degli atteggiamenti, delle relazioni di reciprocità, dell'integrazione socioculturale nel contesto accademico ed extra-accademico, anche in riferimento al territorio; 4) individuare eventuali criticità legate alla cultura organizzativa dell'ateneo in riferimento al successo negli studi dei soggetti di altra cittadinanza e legate al clima sociale nelle situazioni formali ed informali; 5) comparare il campione degli studenti stranieri con un campione di studenti italiani, al fine di rilevare affinità, differenze, percezioni reciproche.

1. Il gruppo di ricerca è così composto: Anna Tozzi - referente di progetto; Alessandro Vaccarelli (docente di Pedagogia interculturale) - coordinamento scientifico; Davie Chuwa, Emblia Nhazai, Valentina Ciaccio, Arianna Fiorenza, Roberto Manzi, Francesco Marola, Oscarine Mbouna, Sonia Pagnanelli, Francesca Palma, Ilaria Salvati - operatori di banca dati e somministratori; Maria Grazia Ferretti e Marta Allevi - collaboratrici per il try-out e la somministrazione; Serena Castellani - supporto elaborazione dati e revisione; Arianna Apicella, Antonio Marangiolo - supporto tecnologico. 
In una prima fase di scouting (novembre 2014) sono stati raccolti dati generali sulla presenza straniera nell'Università dell'Aquila e nelle università italiane, nonché si è proceduto ad un'analisi della letteratura esistente. Alcune prime esplorazioni sono state condotte attraverso una fase qualitativa, effettuata attraverso la raccolta di interviste a soggetti italiani e stranieri e due focus group con studenti di nazionalità miste. Ciò ha consentito di progettare due questionari rigidamente strutturati (fatta eccezione per alcune domande lasciate aperte), da somministrare ad un campione di soggetti italiani e ad un campione di soggetti stranieri. I due strumenti contemplano, al fine di consentire comparazioni, numerose domande comuni (si vedano gli allegati 1 e 2). Sono stati sottoposti a try-out, al fine di perfezionare la loro comprensibilità, la coerenza complessiva della loro "architettura", la coerenza e la copertura semantica delle domande e delle risposte chiuse. I questionari sono stati inseriti su una piattaforma online e sono stati concepiti per essere autosomministrati. Questa modalità ha consentito di raggiungere, attraverso posta elettronica o telefono, anche gli studenti fuori sede e/o non frequentanti. A fronte delle numerose persone che non hanno autocompilato il questionario, si è proceduto anche alla somministrazione telefonica e faccia a faccia. In alcuni casi è stato necessario il ricorso alla lingua inglese. Un quadro delle dimensioni, delle variabili, degli indici esplorati attraverso i questionari sono sintetizzati rispettivamente nelle Figg. 1 e 2. Una versione integrale dei due questionari è contenuta negli Allegati 1 e 2.

\section{Fig. 1 - Le dimensioni e le variabili esplorate nei questionari}

Dati generali: Sesso - Età - Cittadinanza - Pratiche religiose - Residenza, domicilio - Permesso di soggiorno* - Età di arrivo in Italia* - Situazione abitativa - Titolo di studio genitori - Fascia di reddito familiare - Tasse/borse di studio - Eventuale situazione lavorativa. Le competenze linguistiche*: Difficoltà nell'apprendimento dell'italiano - Competenze linguistiche in ingresso e attuali - Corsi e certificazioni linguistiche. Formazione scolastica: Scuola frequentata - Voto diploma - Percezione dell'efficacia della scuola italiana in campo interculturale. Percorso universitario: Corso di laurea e dipartimento - Anno di iscrizione - CFU acquisiti - Voto medio - Informazioni su Univaq - Motivi della scelta di Univaq - Frequenza delle lezioni - Difficoltà nello studio - Tempo dedicato allo studio - Motivazione allo studio - Valutazione della qualità (8 indicatori) - Rapporto con le rappresentanze studentesche - Suggerimenti e proposte. Socialità: Situazione sentimentale - Numero di amici, numero di conoscenti, loro nazionalità - Motivi delle affinità - Luoghi di incontro - Associazionismo. Percezioni e atteggiamenti: Stima della presenza straniera in Italia - Stima della presenza straniera in Univaq - Atteggiamenti colleghi stranieri (14 indicatori) - Atteggiamenti colleghi italiani (14 indicatori) - Atteggiamenti nei confronti degli immigrati (6 indicatori) - Pregiudizi e loro attori. La vita in Italia e all'Aquila: Gradimento dei diversi aspetti della cultura italiana (11 indicatori) - Conoscenza luoghi dedicati ad altre culture - Prospettive di vita in Italia o all'estero - Nostalgia per il Paese di origine* - Valutazione della vita sociale all'Aquila (16 indicatori) - Scala di solidarietà con i problemi del post-terremoto.

* Le dimensioni contrassegnate da asterisco sono state esplorate soltanto per gli studenti di altra cittadinanza 
- Indice di status socio-economico: ottenuto per somma dei punteggi attribuiti al titolo di studio dei genitori (scala da 1 a 6 per madre e scala da 1 a 6 per padre) e al reddito familiare (scala da 1 a 5). Min teor. 3 - Max teor. 17.

- Indice di percezione dell'efficacia della scuola italiana in campo interculturale: ottenuto per somma dei punteggi dei 6 indicatori (scale da 1 a 4) riferiti a lingua italiana, lingue straniere, discipline umanistiche, tecnico-scientifiche, motorie/espressive, integrazione socio-culturale. Min. teor. 6 - Max teor. 24.

- Indice di difficoltà nello studio: ottenuto per somma dei punteggi dei 6 indicatori (scale da 1 a 4) riferiti a uso della lingua negli esami scritti a domanda aperta, a domanda chiusa, uso della lingua negli esami orali, comprensione delle domande negli esami orali, studio dei materiali didattici, comprensione delle lezioni. Min. teor. 6 - Max teor. 24.

- Indice motivazionale: ottenuto per somma dei punteggi dei 6 indicatori (scale da 1 a 4) riferiti a 3 orientamenti di tipo intrinseco e 3 orientamenti di tipo estrinseco. Min. teor. 6 - Max teor. 24.

- Indice valutazione qualità: ottenuto per somma dei punteggi degli 8 indicatori (scale da 1 a 5) riferiti a tutorato, servizi di segreteria, diffusione delle informazioni, didattica, strutture di supporto allo studio, diritto allo studio, logistica, modulistica. Min. teor. 8 - Max teor. 40.

- Indice atteggiamenti nei confronti dei colleghi italiani: ottenuto per somma dei punteggi delle 14 scale di Likert (scale da 1 a 4) riferite ai colleghi italiani. Min teor. 14 - Max teor. 54.

- Indice atteggiamenti nei confronti dei colleghi stranieri: ottenuto per somma dei punteggi delle 14 scale di Likert (scale da 1 a 4) riferite ai colleghi stranieri. Min teor. 14 - Max teor. 54.

- Indice atteggiamenti nei confronti degli immigrati: ottenuto per somma dei punteggi delle 6 scale di Likert (scale da 1 a 4) riferite agli immigrati. Min teor. 6 Max teor. 32.

- Indice di gradimento dei diversi aspetti della cultura italiana: ottenuto per somma dei punteggi attribuiti a 11 indicatori riguardanti la vita politica, l'alimentazione, la vita sociale, ecc. Min teor. 11 - Max teor. 55.

- Indice Valutazione della vita sociale all'Aquila: ottenuto per somma dei punteggi attribuiti a 16 indicatori riguardanti le condizioni abitative, tempo libero, organizzazione del territorio, ecc. Min teor. 16 - Max teor. 80.

\subsection{I campioni studiati}

L'estrazione dei campioni, avvenuta in modo casuale, è stata possibile grazie all'accesso ai database dell'ateneo e ha riguardato gli studenti e le studentesse regolarmente iscritti nell'a.a. 2013/14. Sono stati raggiunti i soggetti che hanno preventivamente autorizzato l'università al trattamento dei dati. In entrambi i casi i campioni soddisfano i criteri per un'ampiezza campionaria con intervallo minimo di confidenza al 95\% e un livello minimo di fiducia fissato a 0,5 .

Per quanto riguarda il campione di studenti con cittadinanza non italiana, sono stati contattati tutti i 561 soggetti appartenenti all'universo degli 
studenti stranieri (iscritti a.a. 2013/14). Il campione raggiunto è composto da 184 soggetti. La stratificazione è stata verifica su area geografica di provenienza e dipartimento di riferimento. Le differenze percentuali indicate nella Tabella 1 indicano, tranne in un caso, scarti inferiori al 5\% rispetto ai dipartimenti frequentati e una distribuzione per aree geografiche di provenienza che tende a sovrarappresentare leggermente gli studenti europei, soprattutto a scapito di quelli asiatici.

Tab. 1 - Confronti universo - campione (studenti con cittadinanza non italiana)

\begin{tabular}{|c|c|c|c|}
\hline Area geografica di provenienza & $\%$ universo & $\%$ campione & Diff. \% \\
\hline Nord Africa & 4,4 & 3,4 & $-1,1$ \\
\hline Africa subsahariana & 13,2 & 12,0 & $-1,2$ \\
\hline Europa occidentale & 14,7 & 19,6 & $+4,9$ \\
\hline Europa balcanica e orientale & 30,0 & 39,6 & $+9,6$ \\
\hline Nord America & 2,9 & 0 & $-2,9$ \\
\hline America centrale e meridionale & 7,4 & 6 & $-1,4$ \\
\hline Medio Oriente & 8,8 & 10,3 & $+1,5$ \\
\hline Asia centro-orientale e sud-orientale & 14,7 & 8,7 & $-6,0$ \\
\hline Dipartimento di riferimento & $\%$ universo & $\%$ campione & Diff. \% \\
\hline $\begin{array}{l}\text { Ingegneria civile, edile-architettura, } \\
\text { ambientale (DICEAA) }\end{array}$ & 5,2 & 8,1 & $+2,9$ \\
\hline $\begin{array}{l}\text { Ingegneria e scienze dell'informazione } \\
\text { e matematica (DISM) }\end{array}$ & 17,6 & 8,7 & $-8,9$ \\
\hline $\begin{array}{l}\text { Ingegneria industriale e dell'informazione } \\
\text { e di economia (DIIE) }\end{array}$ & 11,9 & 13,7 & $+1,8$ \\
\hline $\begin{array}{l}\text { Medicina clinica, sanità pubblica, } \\
\text { scienze della vita e dell'ambiente (MESVA) }\end{array}$ & 49,2 & 52,8 & 3,6 \\
\hline $\begin{array}{l}\text { Scienze cliniche applicate e } \\
\text { biotecnologiche (DISCAB) }\end{array}$ & 3,6 & 1,9 & $-1,7$ \\
\hline Scienze fisiche e chimiche (DSFC) & 0,7 & 1,9 & $-0,2$ \\
\hline Scienze umane (DSU) & 11,8 & 13,0 & $+2,8$ \\
\hline
\end{tabular}

Con un universo di 24.258 studenti (iscritti a.a. 2013/14), il campione di soggetti italiani raggiunto risulta costituito da 398 studenti. La stratificazione è stata verificata su sesso e dipartimento di riferimento. Le differenze ottenute risultano irrilevanti e ciò rende il campione ben stratificato rispetto alle variabili considerate (Tab. 2). 
Tab. 2 - Confronti universo - campione (studenti italiani)

\begin{tabular}{lccc}
\hline Sesso & \% universo & \% campione & Diff. \% \\
\hline Maschi & 55,6 & 55,1 & $-0,5$ \\
Femmine & 44,4 & 44,9 & $+0,5$ \\
\hline Dipartimento di riferimento & \% universo & $\%$ campione & Diff. \% \\
\hline $\begin{array}{l}\text { Ingegneria civile, edile-architettura, } \\
\text { ambientale (DICEAA) }\end{array}$ & 6,7 & 6,5 & $-0,2$ \\
$\begin{array}{l}\text { Ingegneria e scienze dell'informazione } \\
\text { e matematica (DISIM) }\end{array}$ & 7,5 & 7,3 & $-0,2$ \\
$\begin{array}{l}\text { Ingegneria industriale e dell'informazione } \\
\text { e di economia (DIIIE) }\end{array}$ & 21,7 & 21,9 & $+0,2$ \\
$\begin{array}{l}\text { Medicina clinica, sanità pubblica, } \\
\text { scienze della vita e dell'ambiente (MESVA) }\end{array}$ & 37,1 & 38,7 & $+1,6$ \\
$\begin{array}{l}\text { Scienze cliniche applicate e } \\
\text { biotecnologiche (DISCAB) }\end{array}$ & 5,5 & 5,5 & 0 \\
$\begin{array}{l}\text { Scienze fisiche e chimiche (DSFC) } \\
\text { Scienze umane (DSU) }\end{array}$ & 2,1 & 1,8 & $-0,3$ \\
\hline
\end{tabular}

\subsection{Guida alla lettura dei dati}

Il tipo di variabili presenti nel questionario, la tipologia delle domande e delle risposte hanno consentito un approccio di tipo descrittivo, soprattutto per quanto riguarda l'analisi monovariata (frequenze, misure centrali, deviazione standard), e un approccio di tipo esplicativo, che ha permesso di sondare il rapporto tra variabili (analisi bivariata e multivariata). Per l'analisi bivariata sono stati utilizzati diversi test statistici applicati a partire dal tipo di variabile considerata o degli indici costruiti (Laeng, 1992; Lombardo, 1993; Corbetta, 1999):

- per variabili di tipo nominale: test del Chi Quadro e test esatto di Fisher. II Chi Quadro è un test di significatività statistica, applicabile a variabili nominali, volto ad evidenziare se la differenza tra due proporzioni o due percentuali è dovuta o meno al caso, vale a dire se la differenza dei "numeri" tra due gruppi è probabilmente dovuta al caso (test non significativo, con un livello di significatività $>0,05$ ) o no (test significativo, con un livello di significatività $<0,05)$. II test esatto di Fisher viene utilizzato quando entrambe le variabili sono dicotomiche o viene applicato su piccoli campioni. $\mathrm{Si}$ applicano gli stessi criteri per la significatività statistica;

- variabile nominale (indipendente) per variabili ordinali/cardinali: analisi delle medie condizionate e della varianza (Anova). Si tratta di un test utile a confrontare più di due gruppi in rapporto alla media espressa su una variabi- 
le. L'ipotesi nulla si verifica quando le medie dei gruppi sono uguali, mentre l'ipotesi alternativa quando osserviamo una differenza tra gruppi. Se la differenza tra gruppi è accompagnata da un indice $F$ con livello di significatività $\mathrm{P}<0,05$, si può considerare come significativa e dunque non dovuta al caso;

- per variabili cardinali: coefficiente di correlazione parametrico $r$ di Pearson. L'indice di correlazione di Pearson (o di Bravais-Pearson) tra due variabili cardinali è un coefficiente che esprime la linearità tra la loro covarianza e il prodotto delle rispettive deviazioni standard. II rapporto tra le variabili si esprime con un risultato compreso tra -1 e 1 , laddove il segno negativo indica una relazione inversa (al crescere di una variabile decresce il valore dell'altra), il segno negativo indica una relazione diretta (al crescere di una variabile cresce il valore dell'altra), e la prossimità allo 0 orienta verso l'assenza di relazione tra variabili. Anche in questo caso si associa un livello di significatività $P$ che se $<0,05$, può considerarsi soddisfacente;

- variabili ordinali (anche poste in relazione con variabili cardinali): coefficiente di correlazione non parametrico rho di Spearman. Anche in questo caso si associa un livello di significatività $P$ che se $<0,05$, può considerarsi soddisfacente;

- per gli indici sintetici (ottenuti per somma di punteggi scalari): per garantire una maggiore leggibilità dei dati, i punteggi medi ottenuti sugli indici sono stati spesso riportati su una scala 0-10, attraverso la formula: (mediaminimo teorico)/(massimo teorico-minimo teorico) ${ }^{\star} 10$. In questo modo si rende più leggibile il dato rispetto alla distanza tra il minimo e il massimo e si riesce a collocare in maniera più intuiva il valore medio rispetto alle distanze estreme ${ }^{2}$.

Il trattamento statistico dei dati è stato effettuato mediante il programma SPSS. Una prima lettura descrittiva è possibile attraverso l'analisi dei questionari allegati, che riportano le frequenze e i valori riscontrati all'interno di ciascuna variabile.

\subsection{Il doppio binario della presenza straniera all'universi- tà: studenti stranieri scolarizzati in Italia e studenti in- ternazionali. Alcune anticipazioni sui dati}

I dati della ricerca che stiamo per presentare, unitamente alle considerazioni avanzate nella prima parte del volume, di carattere più generale e ri-

2. Ad esempio in un indice con minimo 14 e massimo teorico 56, una media ottenuta di 27,3 viene riscalata, con la formula descritta, con valore 3,1 , che intuitivamente collochiamo su una fascia medio-bassa rispetto alla dimensione considerata. Allo stesso modo, con una media 43,1 otteniamo il valore 6,9 che, sempre intuitivamente, collochiamo su una fascia medio-alta della dimensione considerata. 
ferite al contesto nazionale e internazionale, ci portano a sottolineare la natura marcatamente articolata e complessa della presenza straniera nelle università italiane. Si tratta, infatti, di una presenza che ha origini e radici diverse, non soltanto in riferimento alle provenienze degli studenti e delle studentesse, ma anche rispetto ai motivi, alle cause del loro trovarsi in Italia a compiere gli studi universitari.

Storicamente, una prima linea direttrice che spiega questa presenza, ormai strutturale, va fatta risalire ai flussi esclusivamente legati ai motivi di studio che, già dagli anni ' 50 , vedono l'università attrarre studenti esteri e a definirsi come avanguardia inconsapevole di un multiculturalismo che sarà destinato più avanti ad essere uno degli elementi maggiormente caratterizzanti della società italiana. È stato ricordato come ancora nel 1981, quando il Censimento della popolazione registra un saldo migratorio positivo, con una presenza di stranieri sul territorio nazionale pari allo $0,37 \%$ della popolazione ${ }^{3}$, la percentuale degli studenti stranieri nelle università italiane si attestava al 2,97\%.

Se dagli anni '80 la presenza di studenti di altra cittadinanza è aumentata, sia in termini assoluti sia in termini relativi, fino ad arrivare all'attuale 4,17\% (a.a. 2013/14), è perché una seconda linea direttrice l'ha resa sostanzialmente più dinamica: le migrazioni internazionali e la presenza di minori nelle scuole italiane hanno portato inevitabilmente e con una certa gradualità i figli dei migranti nelle aule accademiche, seppure con difficoltà legate a fattori di contesto legati all'implementazione delle politiche scolastiche, che non sempre consentono ad essi di raggiungere il pieno successo formativo. Facendo riferimento a queste due linee direttrici, abbiamo voluto distinguere tra quegli studenti che scelgono di venire a studiare in Italia da un altro Paese (gli studenti internazionali) e quelli che invece, assimilati ai primi nelle statistiche ufficiali, sono in realtà già presenti in Italia per effetto di precedenti migrazioni familiari, hanno frequentato la scuola italiana, ma hanno una diversa cittadinanza (gli studenti stranieri scolarizzati in Italia).

Alcune anticipazioni sui risultati della ricerca che andremo a leggere nei prossimi capitoli, possono costituire un quadro di riferimento utile a leggere e interpretare al meglio $\mathrm{i}$ dati che verranno esposti in modo più articolato nei seguenti capitoli. Abbiamo infatti riscontrato non soltanto delle differenze significative (rilevate principalmente attraverso il test del Chi Quadro e l'analisi della varianza), nei trend che contraddistinguono i percorsi accademici e le caratteristiche sociali, economiche e culturali degli studenti italiani e di quelli di altra cittadinanza generalmente intesi, ma

3. Fonti: Serie storiche ISTAT e Censimento della Popolazione 1981. 
anche in quelli che, internamente a quest'ultimo gruppo, si riferiscono agli studenti stranieri scolarizzati in Italia e agli studenti internazionali.

Il campione dei 184 soggetti di cittadinanza non italiana si compone del $62,5 \%$ di studenti internazionali e del 37,5\% di studenti stranieri scolarizzati in Italia. Le differenze significative possono essere colte su diverse variabili e fattori, tra i quali quelli più interessanti riguardano il genere, l'età, i fattori socioeconomici di partenza, ma anche gli indicatori di profitto e di integrazione socioculturale. In sostanza possiamo rilevare che per alcuni aspetti gli studenti stranieri scolarizzati in Italia tendono ad avvicinarsi di più alla condizione degli studenti italiani di quanto facciano gli studenti internazionali, che vivono con maggiori ostacoli o difficoltà la loro carriera accademica e l'integrazione sociale. Va sicuramente ricordato, ancora una volta, che i primi accedono all'università con una conoscenza della lingua italiana e della cultura organizzativa dei sistemi formativi, nonché naturalmente dei modelli socioculturali, diversa rispetto a chi, arrivando in Italia specificamente per studiare, vive l'esperienza dello sradicamento, dell'inserimento sociale, talvolta dello shock culturale e linguistico, in modo concomitante all'avvio del percorso di studio. E va ricordato, per una corretta analisi dei dati e per una loro corretta interpretazione, che esistono differenze interne ai due sottogruppi che dipendono da ulteriori variabili prese di volta in volta in considerazione (quali la nazionalità, il tempo trascorso in Italia, il livello di competenza linguistica, ecc.). Tali differenze saranno discusse e approfondite nei capitoli successivi, pertanto la tabella che segue (Tab. 3) vuole rappresentare un momento di inquadramento generale che spiega in modo macroscopico come dentro una rappresentazione unitaria e di senso comune di studente straniero non si possano cogliere quegli elementi che invece caratterizzano fortemente i due sottogruppi, che, lo ripetiamo, hanno dei fattori di background che necessariamente li vanno a distinguere.

Una prima caratterizzazione riguarda le nazionalità, per cui rileviamo che solo alcuni gruppi si distribuiscono (come gli albanesi) tra le due sottocategorie, mentre tendenzialmente troviamo che per alcune nazionalità (greci, israelo-palestinesi e camerunensi) la condizione di studente internazionale è ben definita e costante. L'età media ci porta a constatare che gli studenti internazionali costituiscono il gruppo significativamente più giovane (25,2 anni) anche se confrontato con il campione italiano (26,8 anni), mentre per gli studenti stranieri scolarizzati in Italia, tra i quali sono presenti anche studenti lavoratori, l'età media sale addirittura a 30,9 anni. Ciò anche a fronte del fatto che l'incidenza dei fuori corso pesa notevolmente più sugli studenti internazionali $(40,3 \%)$ che su quelli stranieri scolarizzati in Italia $(16,9 \%)$, che tendono a distinguersi positivamente anche rispetto agli italiani raggiunti $(31,7 \%)$. La distribuzione per genere varia notevol- 
Tab. 3 - Differenze statisticamente significative: studenti italiani e studenti di cittadinanza non italiana (studenti stranieri scolarizzati in Italia e studenti internazionali)

\begin{tabular}{|c|c|c|c|c|}
\hline & Studenti & Studen & di cittadinanza & on italiana \\
\hline \multirow[t]{2}{*}{ Età media } & \multirow[t]{2}{*}{26,8} & \multirow[t]{2}{*}{28,7} & stranieri & 30,9 \\
\hline & & & internazionali & 25,2 \\
\hline \multirow[t]{2}{*}{ Sesso:\% di femmine } & \multirow[t]{2}{*}{$52,3 \%$} & \multirow[t]{2}{*}{$56,5 \%$} & stranieri & $75,4 \%$ \\
\hline & & & internazionali & $46,4 \%$ \\
\hline \multirow{2}{*}{$\begin{array}{l}\text { Reddito familiare: } \\
\% \text { basso }\end{array}$} & \multirow[t]{2}{*}{$13 \%$} & \multirow[t]{2}{*}{$43,5 \%$} & stranieri & $47,7 \%$ \\
\hline & & & internazionali & $41,2 \%$ \\
\hline \multirow[t]{2}{*}{ Prime 5 nazionalità } & \multirow[t]{2}{*}{---------- } & \multirow[t]{2}{*}{$\begin{array}{l}\text { Grecia, } \\
\text { Albania, } \\
\text { Romania, } \\
\text { Israele, } \\
\text { Camerun }\end{array}$} & stranieri & $\begin{array}{l}\text { Albania, } \\
\text { Romania, } \\
\text { Macedonia, } \\
\text { Ucraina, } \\
\text { Moldavia }\end{array}$ \\
\hline & & & internazionali & $\begin{array}{l}\text { Grecia, } \\
\text { Israele, } \\
\text { Camerun, } \\
\text { Albania, } \\
\text { Angola }\end{array}$ \\
\hline \multirow{2}{*}{$\begin{array}{l}\text { Livello di pratica } \\
\text { religiosa (scala 1-10) }\end{array}$} & \multirow[t]{2}{*}{5,7} & \multirow[t]{2}{*}{6,2} & stranieri & 6,5 \\
\hline & & & internazionali & 5,5 \\
\hline \multirow{2}{*}{$\begin{array}{l}\text { Scuola frequentata: } \\
\% \text { formazione liceale }\end{array}$} & \multirow[t]{2}{*}{$66,6 \%$} & \multirow[t]{2}{*}{$63,0 \%$} & stranieri & $53,8 \%$ \\
\hline & & & internazionali & $71,8 \%$ \\
\hline \multirow{2}{*}{$\begin{array}{l}\text { Voto scuola superiore: } \\
\% \text { votazione medio-alta }\end{array}$} & \multirow[t]{2}{*}{$44,2 \%$} & \multirow[t]{2}{*}{$46,8 \%$} & stranieri & $36,5 \%$ \\
\hline & & & internazionali & $55,8 \%$ \\
\hline \multirow{2}{*}{$\begin{array}{l}\text { Conoscenza della lingua } \\
\text { italiana: } \% \text { ottimale }\end{array}$} & \multirow{2}{*}{----------- } & \multirow[t]{2}{*}{$52,0 \%$} & stranieri & $78,5 \%$ \\
\hline & & & internazionali & $36,6 \%$ \\
\hline \multirow[t]{2}{*}{ Incidenza fuori corso } & \multirow[t]{2}{*}{$31,7 \%$} & \multirow[t]{2}{*}{$32,1 \%$} & stranieri & $16,9 \%$ \\
\hline & & & internazionali & $40,3 \%$ \\
\hline \multirow{2}{*}{$\begin{array}{l}\text { Votazione media esami } \\
\text { di profitto }\end{array}$} & \multirow[t]{2}{*}{$25,6 / 30$} & \multirow[t]{2}{*}{$23,8 / 30$} & stranieri & $24,1 / 30$ \\
\hline & & & internazionali & $23,5 / 30$ \\
\hline \multirow{2}{*}{$\begin{array}{l}\text { Corsi a numero } \\
\text { programmato }\end{array}$} & $22,9 \%$ & $55,3 \%$ & stranieri & $36,7 \%$ \\
\hline & & & internazionali & $65,7 \%$ \\
\hline Presenza di & $25,1 \%$ & $53,5 \%$ & stranieri & $47,4 \%$ \\
\hline & & & internazionali & $58,9 \%$ \\
\hline Numero di amici & $44 \%$ & $48,1 \%$ & stranieri & $50,9 \%$ \\
\hline$=0$ inf. a 3 amici & & & internazionali & $45,6 \%$ \\
\hline Numero di conoscenti & $37,9 \%$ & $34,2 \%$ & stranieri & $29,2 \%$ \\
\hline$=0$ inf. a 5 & & & internazionali & $37 \%$ \\
\hline Non hanno amici italiani & $83,9 \% *$ & $19,9 \%$ & stranieri & $9,1 \%$ \\
\hline & & & internazionali & $26,4 \%$ \\
\hline
\end{tabular}


mente nei diversi gruppi: se tra gli studenti internazionali la presenza di donne è del 46,4\% (e ciò andrebbe considerato anche in rapporto ai fattori culturali dei diversi Paesi di provenienza dei soggetti), tra gli studenti stranieri scolarizzati in Italia la presenza femminile sale addirittura al 75,4\%, segno che le ragazze più che i ragazzi che frequentano la scuola italiana tendono ad avere migliori prestazioni e motivazioni a continuare gli studi. Per quanto riguarda la situazione socio-economica, risalta la differente distribuzione tra italiani e non italiani sulle fasce di reddito familiare considerate. In questo caso, vediamo che si colloca ad un livello basso soltanto il 13\% degli italiani, a fronte del 41,2\% degli stranieri scolarizzati in Italia e del 47,7\% degli internazionali. Come si vedrà nei prossimi capitoli, le ragazze e i ragazzi scolarizzati in Italia tendono a compensare tale dislivello soprattutto attraverso forme diverse di attività lavorativa, mentre gli studenti internazionali attraverso l'accesso a borse di studio internazionali.

Considerando, insieme all'incidenza dei fuori corso, altri fattori di rendimento, vediamo che, pur considerando una migliore preparazione in entrata degli studenti internazionali (voto di diploma nella scuola superiore), la media dei voti negli esami di profitto è migliore negli studenti stranieri scolarizzati in Italia, che pure si distanziano per più di un punto in negativo, dagli studenti italiani. Su questo pesa una più sicura competenza linguistica dei soggetti che hanno frequentato la scuola in Italia rispetto a coloro che sono venuti per frequentarvi l'università: il 78,5\% dei primi dichiara di avere un'ottima conoscenza linguistica a fronte del $36,6 \%$ dei secondi. È interessante verificare anche come per la distribuzione delle frequenze per tipo di corso frequentato siano evidenti differenze sostanziali all'interno dei gruppi: se gli italiani frequentano corsi ad accesso programmato (che per la loro natura garantiscono un migliore accesso nel mondo del lavoro) nel 22,9\% dei casi, l'incidenza sale al 36,7\% per gli studenti stranieri scolarizzati in Italia e addirittura al $65,7 \%$ per gli studenti internazionali. Ciò segnala il fatto che per molti studenti figli delle migrazioni la frequenza dell'università è vissuta come investimento e forma di emancipazione sociale ed economica, mentre, per gli studenti internazionali, il carattere di investimento (per quel che comporta studiare all'estero, lontano dalla famiglia, in un Paese con un costo della vita molto spesso superiore a quello del proprio Paese di origine) si fa decisamente più marcato. Come vedremo, sono soprattutto i corsi di laurea ad orientamento sanitario ad attirare studenti di altra cittadinanza.

Generalmente, almeno considerando alcune variabili isolate, gli studenti stranieri scolarizzati in Italia tendono a mostrare maggiori affinità dal punto di vista socio-culturale con gli italiani rispetto agli studenti internazionali: ce lo dimostrano il livello di pratica religiosa (ma, anche come vedremo, una più sottile differenza tra le religioni praticate), alcuni indicatori 
di socialità, come una maggiore propensione ad avere amici e conoscenti al di fuori della propria comunità di appartenenza (non ha amici italiani solo il 7,7\% degli studenti stranieri a fronte del $20,2 \%$ di quelli internazionali); di contro gli studenti internazionali, seppure maggiormente ancorati alla propria comunità e alla rete degli studenti non italiani, mostrano di essere maggiormente espansivi e dunque ad avere un rete di rapporti sociali più ampia, ciò anche in ragione della distanza dai rapporti familiari che tende ad essere compensata da quelli amicali. Colpisce la percentuale decisamente alta di studenti italiani che dichiara di non avere amici di altra nazionalità, pari all' $83,9 \%$ (percentuale che si affievolisce nel caso dei conoscenti ad un $61,5 \%$ che sempre denota una scarsa apertura alla costruzione di una rete sociale di tipo interculturale).

Anche in tema di pregiudizio e discriminazioni, inaspettatamente, abbiamo rilevato, non soltanto una forte presenza di essi in ambito universitario, ma anche una differenza significativa tra i vari gruppi: se gli italiani tendono a sottostimare la loro presenza nell'ambiente accademico $(25,1 \%$ di risposte affermative), gli studenti internazionali (con picchi che colpiscono in modo preponderante gli studenti provenienti dall'Africa subsahariana) riferiscono di essere stati colpiti da pregiudizi e discriminazioni nel $58,9 \%$ dei casi, a fronte di un non meno importante $47,4 \%$ di casi che riguarda gli studenti stranieri scolarizzati in Italia.

Le differenze evidenziate non possono che porre risalto alla necessità di considerare - nella ricerca sul campo, nonché nella rilevazione statistica e nella definizione di politiche formative specifiche - la compresenza di questi due sotto-universi studenteschi, che, pur presentandosi con qualche tratto comune, non sempre pongono bisogni formativi e sociali univoci e convergenti. 


\section{Gli studenti di cittadinanza non italiana all'Università dell'Aquila in relazione al contesto nazionale}

di Serena Castellani

\subsection{Gli studenti di altra cittadinanza in Italia negli ultimi 10 anni}

Sulla base dei dati dell'Anagrafe Nazionale Studenti (Osservatorio MIUR $^{1}$ ), gli studenti di cittadinanza non italiana iscritti nelle varie università italiane sono passati dai 33.032 dell'a.a. 2004/05 ai 70.762 del 2013/14 $(+46,7 \%)$, secondo un incremento costante e progressivo (Graf. 1).

Graf. 1 - Gli studenti di cittadinanza non italiana iscritti nelle università italiane dall'a.a. 2004/05 al 2013/14

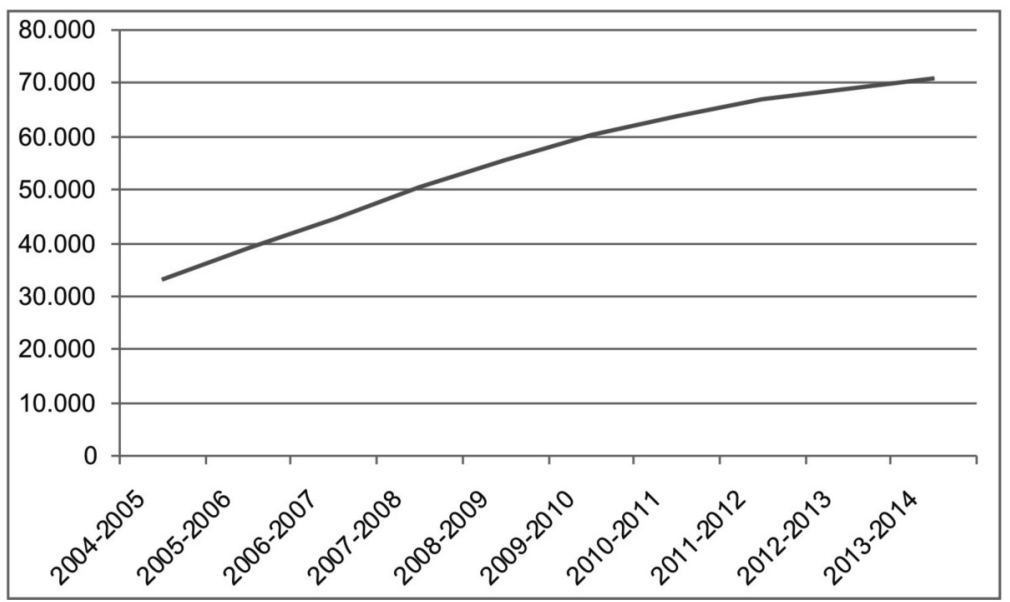

1. Si può consultare la pagina web all'indirizzo http://anagrafe.miur.it/index.php. 
Come mostra il Grafico 2, per ciascun a.a. sono le prime 10 nazionalità a rappresentare la maggior parte di studenti di cittadinanza non italiana iscritti. Ma queste prime 10 nazionalità non restano sempre le stesse nell'arco di tempo preso in considerazione. In effetti, sempre il grafico mostra come possano essere distinte tre categorie di Paesi di provenienza degli studenti:

- quelli che nell'arco di 10 anni risultano sempre tra i primi 10. È il caso di Albania, Romania, Grecia, Camerun e Perù;

- quelli che, dentro il periodo considerato, non risultano più tra i primi $10 . \mathrm{Si}$ tratta di Israele, Polonia, Germania, Croazia e Svizzera;

- quelli che, dentro il periodo considerato, cominciano a risultare tra i primi 10 come la Cina (con una comunità di studenti quantitativamente importante), il Marocco, l'Iran e, molto più recentemente, Moldavia e Ucraina.

Graf. 2 - Le prime 10 nazionalità di studenti di cittadinanza non italiana iscritti (2004-2014)

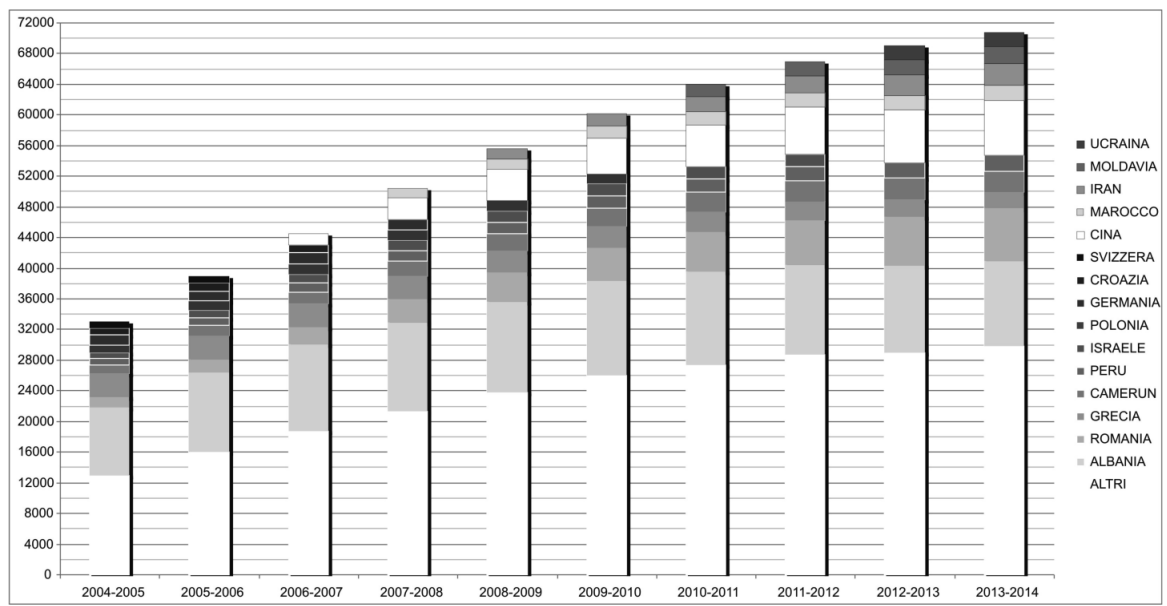

Il dato riportato nel Grafico 2 è confermato anche dal totale degli stranieri residenti in Italia registrato dal XV Censimento della popolazione (2011). In effetti, l'andamento degli studenti di cittadinanza non italiana iscritti nelle università italiane segue, pur non rispecchiando in toto la distribuzione percentuale per nazionalità (cfr. cap. 5), quello del totale della presenza straniera nel nostro Paese. Rispetto al Censimento del 2001 le collettività che hanno avuto dei considerevoli incrementi sono la Romania, l'Albania, il Marocco e la Cina (IDOS, 2014, pp. 89-96). Non a caso, infat- 
ti, gli studenti albanesi iscritti si attestano sempre al vertice della graduatoria; gli studenti rumeni si posizionano tendenzialmente al $3^{\circ}$ posto, quelli marocchini entrano tra le prime dieci posizioni nell'a.a. 2007/08 e si mantengono mediamente tra $1^{\prime} 8^{\circ}$ e il $9^{\circ}$ posto. Attenzione particolare va data agli studenti cinesi i quali entrano tra i primi dieci posti nell'a.a. 2006/07 e fanno registrare un incremento notevole che li posiziona al $2^{\circ}$ posto dall'a.a. 2008/09. Rispetto alla loro presenza, ricordiamo che gli accordi bilaterali tra stati hanno molto incrementato la numerosità degli studenti cinesi (cfr. cap. 3). Il maggiore incremento in percentuale, sempre confrontando il Censimento del 2011 con quello del 2001, lo hanno registrato la Moldavia e l'Ucraina (Ibidem). Questo incremento è testimoniato anche dai dati dell'Osservatorio MIUR sugli studenti di cittadinanza non italiana: dall'a.a. 2010/11 gli studenti moldavi entrano a far parte delle 10 nazionalità più rappresentate nelle università italiane posizionandosi al $10^{\circ}$ posto $\mathrm{e}$ nell'a.a. 2013/14 addirittura al $6^{\circ}$ posto; mentre gli studenti ucraini entrano a far parte dei primi dieci nell'a.a. 2012/13, collocandosi al $10^{\circ}$ posto anche nell'anno successivo.

Se si guarda complessivamente il dato emerso dal Grafico 2 è necessario mettere in evidenza che la nazionalità presente in tutti gli aa.aa., ma che registra un andamento negativo costante e consistente, è la Grecia (cfr. cap. 8). Si tratta di un caso particolare che va analizzato non solo in termini di flussi migratori ma soprattutto considerando il contesto politico, economico e sociale di questo Paese. Il flusso maggiore di studenti greci si registra a cavallo tra gli anni ' 60 e ' 70 con un costante aumento nel corso degli anni ' 70 . Si tratta della generazione cresciuta negli anni della dittatura militare, la quale vedeva questa emigrazione come una trasformazione identitaria (Kornetis, 2007). Nel corso degli anni '80 il flusso migratorio tende a diminuire costantemente nonostante gli studenti greci ammontano a circa il 25\% degli studenti di cittadinanza non italiana presenti in Italia (Papadimitriou, 1997).

Nonostante ciò l'Italia continua ad accogliere molti di coloro che non superano l'esame di ammissione in patria anche se il numero ad oggi si dimostra esiguo. Questa inversione di tendenza dipende sia dalla riforma delle università greche che dalle limitazioni poste dallo stato italiano (Ibidem). Un'idea dell'andamento degli studenti greci iscritti nelle università italiane è illustrata nel Grafico 3. 
Graf. 3 - Studenti di cittadinanza greca iscritti nelle università italiane (aa.aa. $1955 / 56-2009 / 10)^{2}$

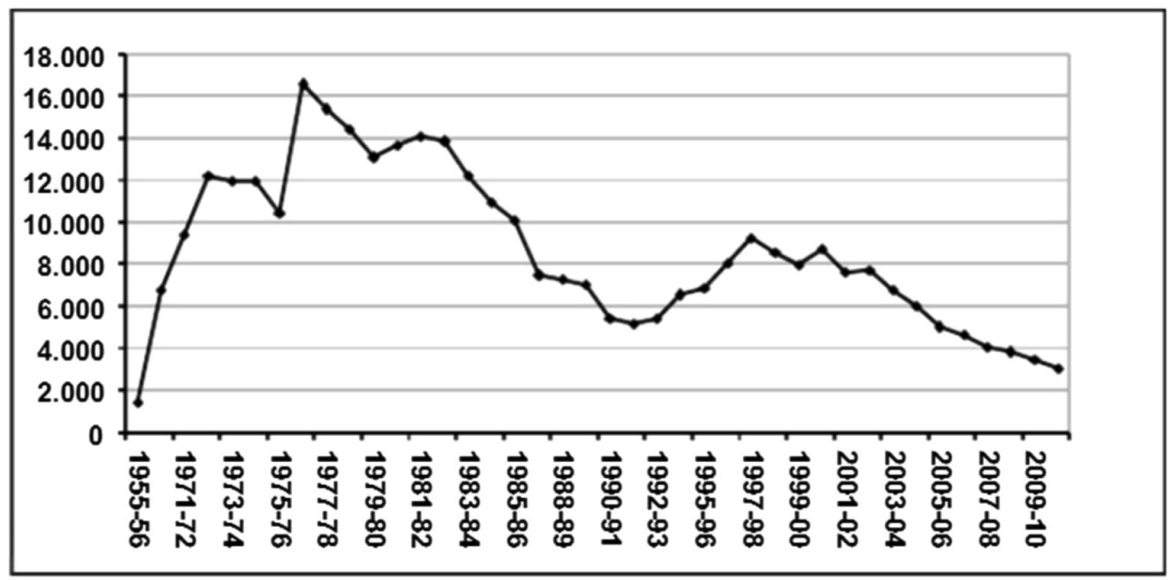

\subsection{La distribuzione su scala regionale}

Continuando con i dati dell'Anagrafe Nazionale Studenti (Osservatorio MIUR), si è analizzata la distribuzione su scala regionale degli studenti di cittadinanza non italiana iscritti nelle varie università italiane dall'a.a. 2004/05 al 2013/14 (Graf. 4).

Analizzando il dato è evidente come nell'arco di 10 anni le regioni Lombardia, Lazio ed Emilia-Romagna accolgano quasi la metà degli studenti di cittadinanza non italiana iscritti nel nostro Paese; inoltre la Lombardia e il Lazio per tutti gli aa.aa. si posizionano sempre, rispettivamente, al primo e al secondo posto, mentre l'Emilia-Romagna si posiziona al terzo posto tranne negli ultimi due aa.aa. in cui viene superata dal Piemonte. Se si analizzano i dati in percentuale si può notare come sia in Lombardia che nel Lazio e in Emilia-Romagna gli studenti di cittadinanza non italiana iscritti crescono in maniera costante e progressiva nel corso di questi 10 anni accademici.

Se si pone l'attenzione sulla regione Abruzzo, dai dati emerge che il numero di stranieri iscritti aumenta costantemente e si aggira intorno alle 1.000 unità fino all'a.a. 2008/09 in cui arriva a 1.175 studenti. Il terremoto del 2009 ha minime ripercussioni su scala regionale rispetto al numero di studenti di cittadinanza non italiana iscritti nell'a.a. 2009/10 nelle univer-

2. Il Grafico 3 è stato estratto dall'articolo di A. Pelliccia (2013). 
Graf. 4 - Numero studenti di cittadinanza non italiana iscritti per ogni regione negli ultimi dieci anni accademici

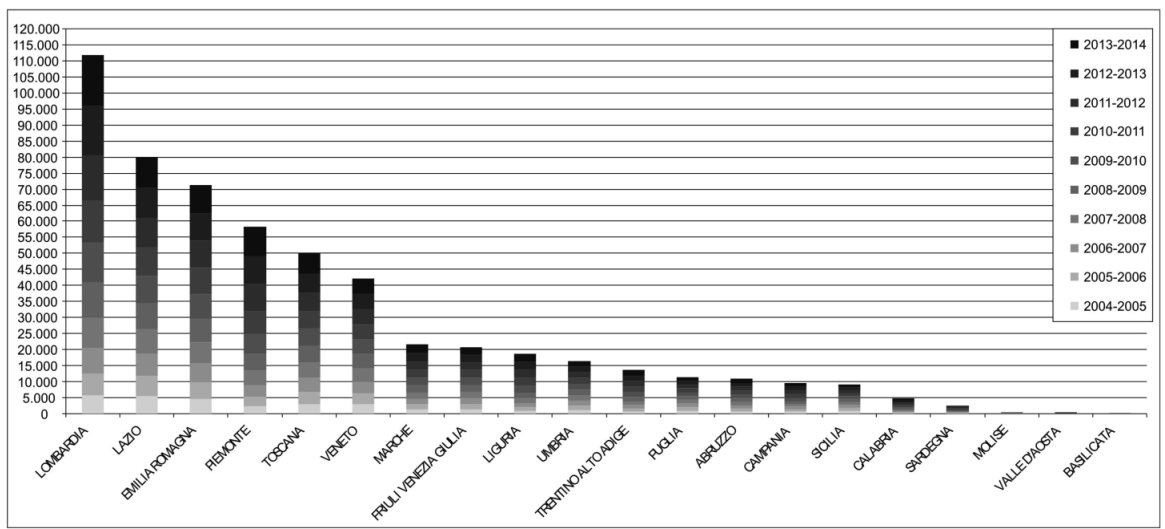

sità abruzzesi (1.151), quindi appena 24 studenti in meno dell'anno precedente. Nei due anni successivi c'è un incremento notevole: 1.235 studenti di cittadinanza non italiana nel 2010/11 e 1.247 nel 2011/12; negli ultimi due anni la presenza degli studenti di cittadinanza non italiana in Abruzzo subisce un calo: infatti, nell'a.a. 2012/13 si iscrivono in 1.204 e nel 2013/14 in 1.199. Soffermarsi all'andamento dei dati della regione Abruzzo è utile per inquadrare il caso dell'Università dell'Aquila che si analizzerà nel paragrafo 4 .

Inoltre, se si mette in relazione il numero degli studenti di cittadinanza non italiana iscritti nelle regioni che si collocano alle prime tre posizioni e i dati delle prime 10 nazionalità rappresentate, emerge che:

- in Lombardia nell'a.a. 2004/05 le nazionalità più rappresentate sono l'Albania, la Svizzera e il Perù; nel 2013/14 sono la Cina, l'Albania e la Romania;

- nel Lazio nel 2004/05 sono l'Albania, la Romania e la Grecia le nazionalità più rappresentate, mentre nel 2013/14 sono la Romania, l'Albania e l'Iran;

- in Emilia-Romagna nel 2004/05 le tre nazionalità più rappresentate sono l'Albania, il Camerun e la Grecia, mentre nel 2013/14 sono l'Albania, la Cina e il Camerun.

\subsection{La distribuzione per atenei italiani}

Oltre alla distribuzione per regioni degli studenti di cittadinanza non italiana si è analizzata la loro distribuzione nei primi 10 atenei italiani, nei quali è rappresentato il maggior numero di iscritti di altra nazionalità nell'arco 
di tempo di 10 anni. Il Grafico 5 mostra che possono essere distinte tre categorie di atenei in cui si iscrivono più studenti di cittadinanza non italiana:

- quelli che nell'arco di 10 anni risultano sempre tra i primi 10. È il caso di Roma "La Sapienza", Bologna, Padova, Firenze, Torino, Milano, Genova e il Politecnico di Milano;

- quelli che da un certo anno in poi non risultano più tra i primi 10. Si tratta di Trieste e Perugia;

- quelli che da un certo anno in poi cominciano a risultare tra i primi 10 come il Politecnico di Torino e Milano-Bicocca.

Graf. 5 - Le prime 10 università italiane con il maggior numero di studenti di cittadinanza non italiana iscritti

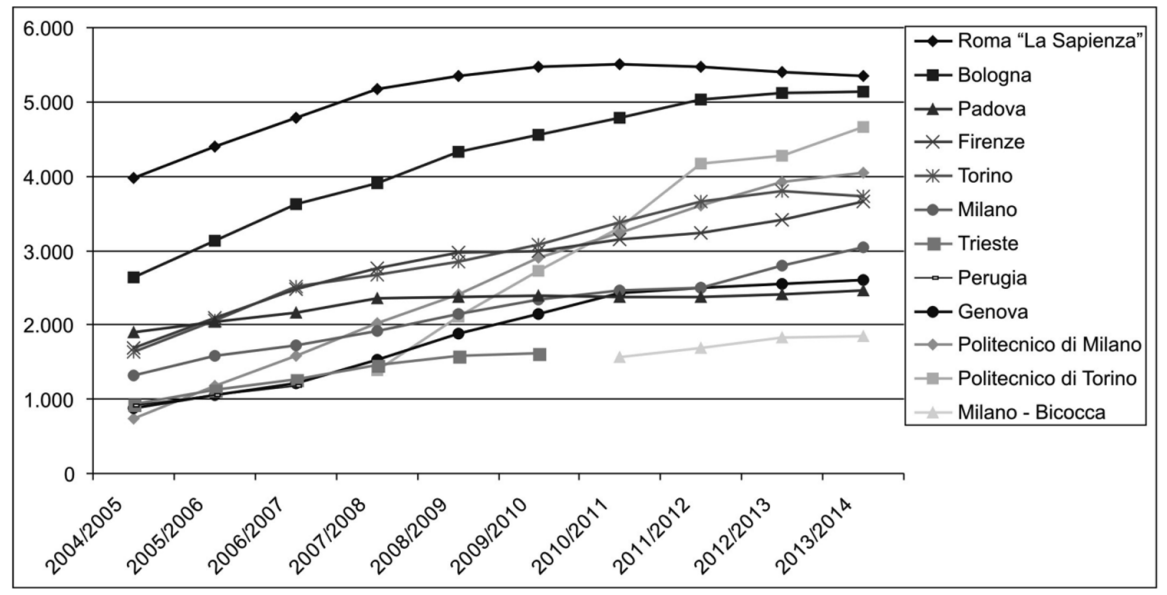

Analizzando il grafico si nota anche che l'Università di Roma "La Sapienza" si posiziona sempre al $1^{\circ}$ posto, così come l'Università di Bologna si attesta sempre al $2^{\circ}$ posto. Inoltre, significativo è l'incremento costante e continuo che registrano i Politecnici di Milano e di Torino.

Se si pone uno sguardo alle università abruzzesi si vede come L'Aquila e Chieti-Pescara nel corso degli ultimi dieci anni si intervallano tra il $30^{\circ} \mathrm{e}$ il $38^{\circ}$ posto in graduatoria, mentre l'Università di Teramo si posiziona tendenzialmente tra il $50^{\circ}$ e il $60^{\circ}$ posto. L'Università dell'Aquila passa dai 357 studenti di cittadinanza non italiana nel 2004/05 ai 564 nel 2013/14; il dato nel corso degli anni è abbastanza oscillante ma nell'a.a. 2009/10 conosce una vera e propria battuta d'arresto, sicuramente legata all'evento sismico che ha colpito L'Aquila nell'aprile 2009 (476 iscritti contro 515 dell'anno precedente). 


\subsection{Gli studenti di cittadinanza non italiana verso l'Uni- versità dell'Aquila negli ultimi 10 anni}

Grazie ai dati dell'Osservatorio MIUR è possibile esaminare l'andamento degli studenti di cittadinanza non italiana iscritti all'Università dell'Aquila dall'a.a. 2004/05 all'a.a. 2013/14. L'accesso ai dati in possesso dell'ateneo aquilano ha permesso di fare un confronto con i dati online del Ministero: si registra una lieve discrepanza poiché l'Università dell'Aquila, rispetto al totale degli iscritti di altra cittadinanza, comprende anche coloro che hanno aderito ai programmi di scambio e gli iscritti ai corsi postlaurea (master, dottorato di ricerca, scuole di specializzazione), a differenza del MIUR che comprende solo gli iscritti ai corsi di laurea di I e II livello e a ciclo unico (Graf. 6).

Graf. 6 - Confronto dati MIUR e Univaq sugli studenti di cittadinanza non italiana iscritti all'Università dell'Aquila

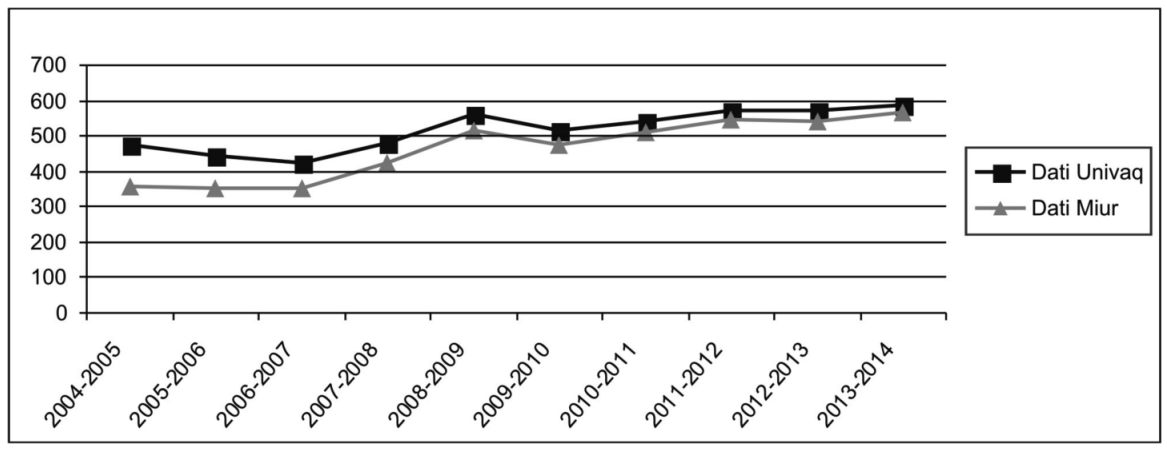

Gli studenti di cittadinanza non italiana iscritti all'Università dell'Aquila rappresentano, rispetto al totale degli studenti iscritti nelle università italiane appartenenti a questa categoria, una percentuale che si aggira intorno al $2 \%$, nell'arco dei 10 anni accademici presi in esame.

Le tre nazionalità che rappresentano più del $50 \%$ degli iscritti all'Università dell'Aquila dall'a.a. 2004/05 all'a.a. 2011/12 sono Grecia, Albania e Israele. La Romania sale alla terza posizione prendendo il posto di Israele negli anni accademici 2006/07 e 2013/14; mentre nel 2011/12 si posiziona sempre al $3^{\circ}$ posto ma a pari merito con l'Albania. Negli ultimi due anni accademici la percentuale delle prime tre nazionalità scende leggermente al dì sotto del $50 \%$, precisamente al $44 \%$, rispetto al totale degli iscritti di altra cittadinanza all'ateneo aquilano. Il quadro attuale, riferito all'a.a. 
2013/14, mostra che le prime dieci nazionalità maggiormente rappresentate coprono il $60 \%$ dell'universo di studenti di cittadinanza non italiana. Nello specifico: Grecia (18,7\%), Albania (13\%), Romania (11,2\%), Israele (10\%), Ucraina (4\%), Moldavia (2,7\%), Iran (2,5\%), Polonia (2,5\%), Venezuela $(1,8 \%)$.

Il Grafico 7 riassume quanto detto sinora e permette di fare ulteriori tre significative considerazioni sulla Grecia:

- essa si posiziona per tutti i dieci aa.aa. analizzati al primo posto della graduatoria;

- dall'a.a. 2004/05 all'a.a. 2013/14 il numero degli studenti greci iscritti all'Università dell'Aquila subisce un calo continuo e progressivo, tanto che passa dai 271 iscritti ai 105;

- la percentuale degli studenti greci all'Università dell'Aquila, rispetto al totale degli iscritti di cittadinanza non italiana, si attesta tra il 2004/05 e il $2007 / 08$ al dì sopra del 50\%; dato che tende a diminuire costantemente e rappresentare, quindi, un terzo del totale degli iscritti e negli ultimi due anni addirittura meno di un quarto.

Graf. 7 - Le nazionalità più rappresentate all'Università dell'Aquila (2004-2014)

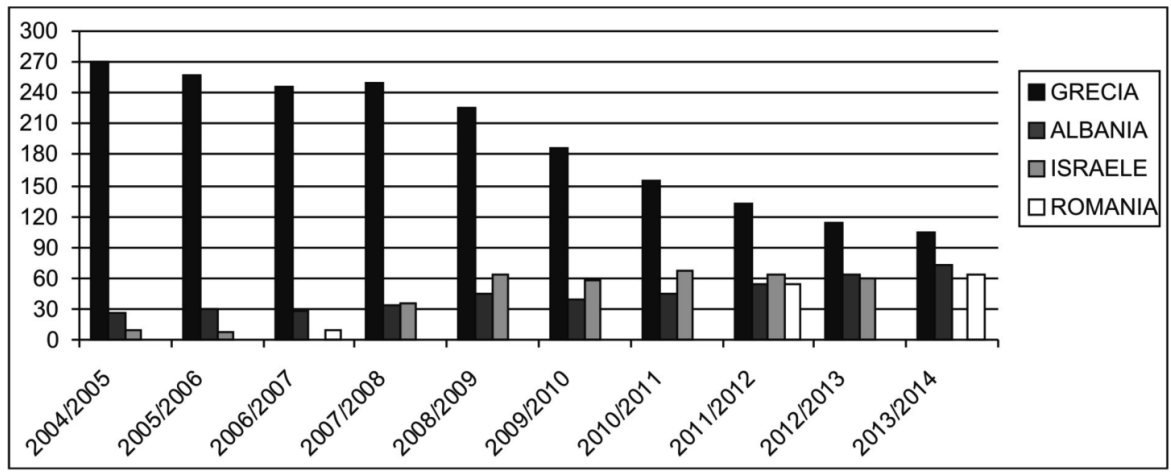

I dati messi a disposizione dall'Osservatorio MIUR, oltre che permettere di ottenere il totale degli studenti di altra cittadinanza iscritti all'Università dell'Aquila e rintracciare le nazionalità più rappresentate, consente anche di reperire per ogni ateneo il dettaglio delle facoltà (ormai ex facoltà) alle quali gli studenti di cittadinanza non italiana si sono iscritti nell'arco temporale degli ultimi dieci anni.

Ciò ha permesso di individuare a quali facoltà decidono di iscriversi gli studenti di altra cittadinanza. Il Grafico 8 mostra che la facoltà che accoglie il maggior numero di iscritti di altra cittadinanza e che si colloca sem- 
pre al $1^{\circ}$ posto è Medicina e Chirurgia, seguita stabilmente al $2^{\circ}$ e $3^{\circ}$ posto, rispettivamente, da Ingegneria e da Scienze Matematiche, Fisiche e Naturali.

Significativo è il dato riguardante la Facoltà di Psicologia, la quale registra un aumento evidente degli iscritti che nel giro di qualche anno la colloca tra il $4^{\circ}$ e il $5^{\circ}$ posto, superando in alcuni anni accademici il numero di iscritti della Facoltà di Lettere e Filosofia che tendenzialmente si situa al $4^{\circ}$ posto.

Graf. 8 - La percentuale degli studenti di cittadinanza non italiana iscritti nelle diverse facoltà dell'Università dell'Aquila

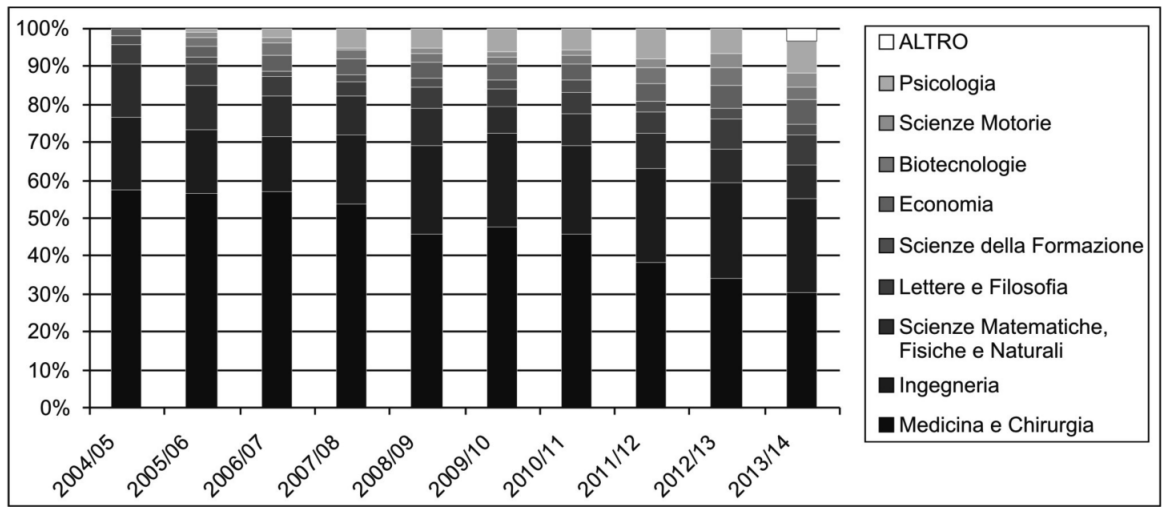

Per concludere, l'Università dell'Aquila è un ateneo che nel corso degli ultimi dieci anni accademici:

- ha immatricolato un numero limitato di studenti di cittadinanza non italiana rispetto al totale degli iscritti (il $2 \%$ contro una tendenza nazionale del $4,7 \%$ - a.a. 2013/14);

- ha una significativa tradizione di accoglienza di studenti di nazionalità greca (seppure in decrescita) e mediorientale;

- ha fatto registrare almeno il $50 \%$ degli iscritti di altra cittadinanza alla Facoltà di Medicina e Chirurgia fino all'a.a. 2007/08 e nel corso dei successivi anni accademici tale percentuale è diminuita fino a toccare il $30 \%$, riportando un costante aumento degli iscritti in tutte le altre facoltà dell'ateneo. 


\title{
8. Gli studenti italiani e non: chi sono
}

\author{
di Francesco Marola, Sonia Pagnanelli
}

\subsection{Introduzione}

Le informazioni che prenderemo in esame e metteremo a confronto in questo capitolo riguardano diverse caratteristiche dei soggetti intervistati, nello specifico: il sesso, l'età, la cittadinanza (relativamente al campione di studenti con nazionalità non italiana), la residenza e la condizione abitativa, la religione e il livello di osservanza delle pratiche religiose, le situazioni economiche e i background socio-culturali. In questo modo sarà possibile ottenere un ritratto minimo della condizione di provenienza dei soggetti che sono stati raggiunti dalla nostra indagine.

\subsection{Il sesso e l'età}

In entrambi i campioni esaminati, la maggioranza degli studenti è composta da donne; su 398 italiani le donne sono 220 (55,3\%), mentre gli uomini sono $178(44,7 \%)$. Su un totale di 184 studenti con cittadinanza non italiana, le donne sono 104 (56,5\%) e gli uomini 80 (43,5\%). Riscontriamo dunque, nella comparazione tra i campioni, una distribuzione sostanzialmente omogenea.

Altra informazione che contribuisce alla messa a fuoco della fisionomia generale dei due gruppi di studenti è l'età, ma la presentazione di questo dato necessita di una premessa. L'Università dell'Aquila è stata interessata, per i cinque anni successivi al terremoto del 2009, dalla riduzione delle tasse universitarie, sia per gli studenti già iscritti sia per quelli immatricolati negli anni in questione, per far fronte alla situazione di disagio in cui

1. I paragrafi 8.2., 8.3., 8.4. e 8.5. vanno attribuiti a Francesco Marola, mentre il paragrafo 8.6. a Sonia Pagnanelli. 
versava la città e alla relativa mancanza di servizi e di strutture adeguate alle necessità degli studenti, che avrebbero potuto causare un forte calo nelle iscrizioni. Si può riscontrare che ciò ha avuto principalmente due effetti sul corpus degli iscritti: da un lato ha permesso che si contenesse il calo delle iscrizioni e delle immatricolazioni, dall'altro ha fatto sì che l'età media degli studenti si alzasse notevolmente, con l'iscrizione di numerose persone già laureate o di lavoratori. L'età media degli studenti italiani raggiunti è pari a 28,7 anni; invece la media che si ottiene dai dati del gruppo degli studenti con altra cittadinanza è di 26,8 anni. Pertanto gli studenti italiani presentano un'età media maggiore di 2 punti rispetto quelli di altra cittadinanza: quest'ultima è dunque più giovane della popolazione italiana. La deviazione standard è sostanzialmente assimilabile tra i due campioni (italiani: 7,6; stranieri: 7,8) e indica che la differenza media è statisticamente significativa ${ }^{2}$.

\subsection{La provenienza geografica}

Il quarto quesito rivolto agli studenti con cittadinanza non italiana prende in esame la loro provenienza. Essendo le risposte molto variegate è necessario tralasciare il dato delle specifiche nazionalità (a meno che si tratti di risposte rilevanti, capaci di raggruppare un ampio numero di soggetti) ed effettuare una schematizzazione delle principali macro-aree geografiche di provenienza (Tab. 1).

Tab. 1 - La provenienza geografica

\begin{tabular}{lc}
\hline Area geografica & Studenti con cittadinanza non italiana \\
\hline Africa subsahariana & $11,4 \%$ \\
\hline Africa settentrionale & $3,3 \%$ \\
\hline America centrale e meridionale & $6 \%$ \\
\hline Asia centro-orientale & $6,5 \%$ \\
\hline Asia sud-orientale & $2,2 \%$ \\
\hline Europa balcanica e orientale & $39,6 \%$ \\
\hline Europa occidentale & $19,6 \%$ \\
\hline Medio Oriente & $10,3 \%$ \\
\hline
\end{tabular}

2. L'analisi della varianza restituisce un alto livello di significatività statistica $(0,001)$. 
Le informazioni indicano innanzitutto che la maggior parte dei soggetti (109) proviene dal continente europeo, con una percentuale pari al $59,2 \%$ delle risposte. Il continente africano è la seconda area per provenienza, con una percentuale del 14,7\% degli studenti; seguono il Medio Oriente, con il 10,3\%, l'Asia con il 8,7\%, l'America centrale e meridionale con il $6 \%$.

Procedendo ad un'osservazione più ravvicinata delle informazioni, distingueremo, per quanto riguarda gli studenti di origine europea, tra chi proviene dai Paesi dell'Europa occidentale e chi da Paesi dell'Europa balcanica e orientale, indipendentemente dall'appartenenza o meno di questi ultimi all'Unione Europea.

La prima macro-area del Vecchio Continente per provenienza è l'Europa balcanica e orientale, luogo di origine di 73 studenti (39,6\%). Al suo interno gli stati più largamente rappresentati sono l'Albania e la Romania, in entrambi i casi con un $12,5 \%$. Contrariamente all'andamento del dato relativo all'afflusso decrescente di studenti greci, quello riguardante gli studenti albanesi e romeni mostra un'ascesa nel corso degli anni. Anche l'Ucraina e la Polonia sono Paesi d'origine rilevanti, rispettivamente indicate nel $3,8 \%$ e $3,2 \%$ delle risposte.

Provengono dagli stati dell'Europa occidentale 36 studenti (19,6\% del totale), con una prevalenza di greci, 31 risposte $(16,8 \%)$. È da notare che il numero degli studenti greci è in calo rispetto agli anni precedenti (cfr. supra, Castellani).

All'interno del gruppo di 27 studenti che provengono dal continente africano $(14,7 \%)$, è da osservare come la gran parte - 21 studenti $(11,4 \%)-$ provenga da stati dell'Africa subsahariana. Tra di essi, 7 sono originari del Camerun $(3,8 \%)$ e i restanti si dividono tra Angola, Tanzania, Madagascar, mentre il Nord Africa (rappresentato da studenti egiziani, marocchini, tunisini e libici) è stato indicato soltanto in 6 risposte $(3,3 \%)$.

Dal Medio Oriente (19 risposte) proviene il 10,3\% degli studenti, principalmente da Israele (10 studenti israelo-palestinesi), seguito da Libano, Iran e Turchia.

Per quanto riguarda l'Asia centro-orientale, da cui provengono 16 studenti $(8,7 \%)$, vediamo che il dato preponderante riguarda l'India con 5 intervistati (2,7\%); invece il Sud-Est asiatico (Filippine, Thailandia, Indonesia) è emerso in 4 risposte $(2,2 \%)$.

Da ultimo, il dato sui Paesi dell'America centrale e meridionale, che riguarda 11 studenti (6\%): in questo caso sono maggiormente rappresentati gli stati di Colombia e Venezuela (4 studenti - il 2,2\% - provenienti da ciascuno dei due). 


\subsection{Provincia di residenza e condizione abitativa}

I dati che riguardano la provincia di residenza in Italia dei due campioni di studenti sono estremamente variegati, in quanto le informazioni ottenute si sono rivelate molto eterogenee; tuttavia l'area geografica preponderante nelle risposte di entrambi i gruppi è quella delle regioni centro-meridionali, con netta preminenza numerica di studenti residenti nelle province abruzzesi.

La prima provincia per rilevanza è quella dell'Aquila, per quanto riguarda sia gli studenti italiani che gli studenti di cittadinanza non italiana: il $38,8 \%$ dei primi e il $61 \%$ dei secondi dichiara infatti di risiedervi, con il dato percentuale relativo agli studenti con cittadinanza non italiana nettamente superiore al primo.

Nel dato di entrambi i campioni le altre province abruzzesi seguono con andamento simile per rilevanza. L'11,5\% degli italiani e il 12\% degli stranieri risiedono a Teramo e provincia, che emerge come la seconda zona di residenza per i due gruppi. Lo scarto percentuale tra essi aumenta, tuttavia, nel caso della provincia di Pescara, terza per numero di risposte nel campione di cittadini italiani (con il 7,5\%), meno importante invece in quello di studenti con cittadinanza non italiana, dove è stata indicata nel $2 \%$ delle risposte. Analogo il caso di Chieti, infatti, tra gli italiani, la quota sul totale è del 5,5\% contro quella del $2 \%$ relativa agli studenti stranieri.

Roma emerge in un numero considerevole di risposte nel primo come nel secondo campione, rappresentando rispettivamente il $6 \%$ e il $7 \%$; la capitale risulta dunque essere la terza provincia quanto al dato di residenza per gli studenti provenienti dall'estero che hanno preso parte all'indagine. Le altre province laziali hanno una certa occorrenza nelle risposte, ma con netta differenza percentuale tra gli studenti italiani e quelli internazionali; se Rieti segue Roma tra le città di residenza sia per quanto riguarda il primo campione (5,8\% delle risposte), che per quanto riguarda il secondo (3\%), Frosinone e Latina, province in cui complessivamente risiede il 3,8\% degli studenti italiani, non emergono invece con rilevanza particolare tra le risposte di quelli di cittadinanza non italiana.

Proseguendo con l'analisi possiamo riscontrare una progressiva dispersione del dato tra città marchigiane (la provincia di Ascoli Piceno raccoglie l'1\% italiano e il $2 \%$ internazionale), campane e pugliesi - particolarmente rappresentate nel campione di italiani - mentre nel campione degli studenti non italiani compaiono anche province di residenza estere, per esempio greche ( $1 \%$ delle risposte).

Per concludere presentiamo l'esame del dato che riguarda la condizione abitativa (Tab. 2). 


\begin{tabular}{lcc}
\hline & Studenti italiani & $\begin{array}{c}\text { Studenti con } \\
\text { cittadinanza } \\
\text { non italiana }\end{array}$ \\
\hline Casa a L'Aquila con famiglia & $17,6 \%$ & $14,4 \%$ \\
\hline Casa in affitto con altri studenti & $35,9 \%$ & $41,7 \%$ \\
\hline Pendolarismo & $38,2 \%$ & $37,4 \%$ \\
\hline Residenza universitaria & $2,5 \%$ & $6,4 \%$ \\
\hline Altro & $5,8 \%$ & $0,7 \%$ \\
\hline
\end{tabular}

Per quanto riguarda la condizione abitativa assieme ad altri studenti, si può riscontrare una prevalenza tra le risposte degli studenti con cittadinanza non italiana: il 41,7\% dichiara di condividere una casa in affitto con altri studenti e il $6,4 \%$ vive in una residenza universitaria, mentre tra gli italiani le percentuali scendono al $35,9 \%$ e $2,5 \%$. Situazione opposta per la condizione abitativa con la propria famiglia all'Aquila, indicata dal 17,6\% degli italiani, mentre dal $14,4 \%$ degli studenti non italiani. Un dato sostanzialmente omogeneo riguarda invece il pendolarismo, indicato dal 38,2\% degli italiani e dal $37,4 \%$ dei non italiani.

Un dato particolarmente interessante per la nostra indagine, non indicato nella Tabella 2, riguarda gli studenti italiani che vivono in una casa in affitto: il $12 \%$ di loro dichiara di condividere l'appartamento con almeno uno di altra cittadinanza.

\subsection{Le pratiche religiose}

Agli intervistati è stato chiesto di indicare in primo luogo se fossero o meno appartenenti a un credo religioso; il quesito successivo, invece, chiedeva di indicare il grado di osservanza su una scala discendente che va da "molto" a "per niente". Vedremo come le risposte omesse nel secondo dei quesiti corrispondano coerentemente al numero di studenti di entrambi i gruppi che hanno precedentemente dichiarato di non essere religiosi, e come il dato che emerge rispetto alla pratica quotidiana della religione sia significativamente diverso tra un gruppo e l'altro (Tab. 3).

Il campione italiano in netta maggioranza esprime appartenenza alla religione cristiana; il dato riguarda infatti 281 soggetti, ovvero il 70,6\% del totale.

La religione cristiana è quella largamente maggioritaria anche all'interno del campione di studenti con cittadinanza diversa da quella italiana, in- 
dicata, infatti, da 116 soggetti (il $63 \%$ del totale). Non abbiamo distinto ulteriormente tra le varie confessioni delle diverse religioni per esigenze di sintesi.

Tab. 3 - Le pratiche religiose

\begin{tabular}{lrc}
\hline Religione & $\begin{array}{c}\text { Studenti } \\
\text { italiani }\end{array}$ & $\begin{array}{c}\text { Studenti con } \\
\text { cittadinanza } \\
\text { non italiana }\end{array}$ \\
\hline Cristiana & $70,6 \%$ & $63 \%$ \\
\hline Musulmana & $0 \%$ & $20,6 \%$ \\
\hline Buddista & $1,3 \%$ & $0,5 \%$ \\
\hline Induista & $0 \%$ & $1,6 \%$ \\
\hline Bantu & $0 \%$ & $0,5 \%$ \\
\hline Ebraica & $0,3 \%$ & $0 \%$ \\
\hline Nessuna & $24,3 \%$ & $14,1 \%$ \\
\hline Altro & $3,5 \%$ & $0 \%$ \\
\hline
\end{tabular}

Il secondo dato per rilevanza complessiva è quello che concerne la non appartenenza ad alcun credo religioso: nel campione degli studenti italiani la percentuale è del $24,3 \%$ con 97 risposte in tal senso, laddove in quello degli studenti di altra cittadinanza è del 14,1\%. Sebbene le percentuali varino di circa 10 punti tra il gruppo di italiani e il gruppo di non italiani, ad indicare quanto i primi siano complessivamente meno religiosi dei secondi, la presenza di soggetti non religiosi emerge con numeri importanti in entrambi i campioni, costituendo infatti il secondo dato assoluto sulla somma dei due gruppi.

La religione musulmana emerge con una forte percentuale nelle risposte degli studenti di altra cittadinanza (38 risposte), costituendo il 20,6\% del totale. Questo dato segna una differenza importante con il gruppo italiano, nel quale nessun soggetto dichiara di essere musulmano.

Per finire, riportiamo il dato che riguarda l'appartenenza a religioni diverse dalla cristiana e musulmana, relativamente ai casi più significativi: notiamo quindi che un piccolo numero di studenti di entrambi i campioni (5 italiani e 1 straniero) si dichiara buddista; la religione induista riceve invece 3 risposte nel gruppo degli studenti con cittadinanza non italiana; infine, la religione ebraica viene indicata solamente da parte di uno studente italiano, quella bantu da un non italiano.

Incrociando il dato relativo alla confessione religiosa con quello inerente la provenienza geografica possiamo notare che la religione cristiana è lar- 
gamente maggioritaria tra gli studenti della macro-area dell'Europa occidentale, indicata da 31 studenti su 36.

Allo stesso modo la religione cristiana è prevalente nell'area dell'Europa balcanica e orientale, da cui provengono 73 studenti. I cittadini di Paesi quali Romania, Ucraina e Polonia si dichiarano tutti cristiani (per un totale di 36 risposte), mentre gli studenti musulmani originari della stessa macro-area vengono principalmente dalla Macedonia e dall'Albania; tuttavia, bisogna sottolineare come la maggioranza degli albanesi intervistati si dichiari atea (11 su 23).

Anche tra gli studenti dell'Africa subsahariana la religione cristiana è preponderante, infatti è stata indicata in ben 15 risposte su 21 , laddove i rimanenti 6 intervistati si dividono tra 3 musulmani, 2 atei e 1 bantu.

Si può presumere che una buona parte dei soggetti appartenga a confessioni cristiane diverse da quella cattolica: la confessione ortodossa, per esempio, è predominante in Grecia (in cui ricordiamo avere la cittadinanza 31 intervistati) così come in Romania (di cui sono cittadini 23 tra coloro che hanno preso parte all'indagine) e infatti essa è sporadicamente specificata alla voce "Altro" del questionario (come fatto da 5 intervistati del continente europeo).

Come abbiamo visto, oltre ai dati sui Paesi dell'Europa balcanica e orientale quali l'Albania, la Macedonia, il Kosovo, la religione musulmana emerge in maniera preponderante in quelli relativi a macro-aree come il Medio Oriente (12 musulmani a fronte di 6 cristiani), e il Nord Africa (5 studenti su 6 appartenenti al credo islamico, a fronte di un solo cristiano).

Con andamento conforme al quesito precedentemente preso in esame, alla domanda "Sei un credente praticante?" vediamo scendere il numero dei rispondenti a 300 italiani e a 158 studenti di altra cittadinanza. In questo caso, l'aspetto più rilevante che emerge dal complesso delle risposte è che gli studenti non italiani risultano mediamente più osservanti (così come erano risultati complessivamente più credenti) delle pratiche religiose rispetto agli studenti italiani.

È, infatti, superiore a quella degli italiani la percentuale degli studenti di origine estera che riempiono le voci "molto" (il 15,7\% contro l'11,3\%) e "abbastanza" (il 31,9\% contro il 27,2\%); mentre alle voci "poco" e "per niente" si presenta la situazione opposta: rispettivamente il $38,5 \%$ e il $22,9 \%$ degli italiani si contrappone al $36,3 \%$ e al $16,4 \%$ dei non italiani. Nel complesso, si può riscontrare che la risposta principalmente indicata è "poco", in entrambi i campioni. 


\subsection{Gli studenti di cittadinanza non italiana: studenti in- ternazionali e studenti scolarizzati in Italia}

Parlare di studenti di cittadinanza non italiana, in alternativa al termine più impreciso di stranieri, risulta essere una scelta che rende maggiormente giustizia alla composizione eterogenea degli iscritti e permette di sottolineare una distinzione che non è utile minimizzare: il panorama degli universitari composto dai cittadini non italiani comprende, infatti, sia coloro che hanno scelto di iscriversi all'Università dell'Aquila per motivi strettamente inerenti alla loro formazione culturale, sia i figli di immigrati, che risiedono in questo Paese insieme alle loro famiglie. I primi sono quindi tendenzialmente migranti provvisori provenienti da diverse aree geografiche, i secondi concludono un percorso formativo già precedentemente iniziato nella scuola italiana e probabilmente contribuiranno, più dei primi, a connotare il futuro livello socio-culturale e anche economico-politico di questo Paese.

Frazionando la percentuale complessiva degli intervistati che non hanno cittadinanza italiana (Tab. 4), si riesce a mettere in evidenza come varia il dato percentuale tra coloro che frequentano l'Università dell'Aquila per una scelta mirata (studenti internazionali) e coloro che hanno frequentato già la scuola in Italia (studenti stranieri scolarizzati in Italia): i primi costituiscono il $62,5 \%$ del campione, i secondi il $37,5 \%$.

La presenza di studenti internazionali è senza dubbio prevalente, in controtendenza con una stima (cfr. cap. 5) che rileva, durante gli ultimi anni, una crescita della percentuale straniera nella scuola e che indurrebbe a pensare ad una realtà quantomeno più proporzionata tra studenti provenienti da realtà internazionali e stranieri che vivono in Italia.

Una differenza significativa tra i due gruppi la individuiamo già in merito all'età: all'interno del campione di studenti con cittadinanza non italiana l'età media risulta di 25,2 anni tra gli studenti internazionali e di 30,9 anni tra gli studenti stranieri scolarizzati in Italia, con un'analisi della varianza fortemente significativa $(\mathrm{P}=0.000)$. Ciò lascia supporre che per gli studenti stranieri l'incontro con l'università non sia sempre vissuto in maniera lineare e che, in alcuni casi, forse anche per le politiche di esenzione dalle tasse nel post-sisma aquilano, il percorso sia stato intrapreso anche da lavoratori/lavoratrici migranti adulti.

Come abbiamo visto, su un campione di 185 iscritti di nazionalità non italiana, una percentuale che supera abbondantemente la metà $(56,7 \%)$ è costituita da cittadini europei. Per il continente africano, la regione subsahariana pesa per circa il $12 \%$ sul numero degli iscritti di origine estera, mentre l'apporto dei Paesi nordafricani è lievemente al di sopra del 3\%. 
Dal Medio Oriente, in particolare da Israele e dal Libano, proviene quasi il $10 \%$ del campione e dall'Asia centro-orientale e sud-orientale poco più dell' $8 \%$. L'America centrale e meridionale è indubbiamente rappresentata con una percentuale di nativi più cospicua di quella relativa al Nord America (rispettivamente $4,8 \%$ e $0,5 \%$ ).

Procedendo ad un'analisi più approfondita, si arriva a focalizzare inevitabilmente l'attenzione su un dettaglio: per ognuna delle diverse aree geografiche di provenienza, la percentuale degli intervistati che dichiara di essere in Italia esclusivamente per motivi di studio non solo è più alta rispetto alla percentuale degli scolarizzati, ma in alcuni casi è pressoché totalizzante. In questo quadro generale, l'unica eccezione che riporta una tendenza contraria è l'area dell'Europa balcanica.

Tab. 4 - Le provenienze geografiche degli studenti internazionali e degli studenti stranieri scolarizzati in Italia

\begin{tabular}{lrrr}
\hline & $\begin{array}{c}\text { Studenti stranieri } \\
\text { scolarizzati in Italia }\end{array}$ & $\begin{array}{c}\text { Studenti } \\
\text { internazionali }\end{array}$ & Totale \\
\hline Africa subsahariana & 2 & 20 & 22 \\
\hline America centrale e meridionale & $9 \%$ & $91 \%$ & $100 \%$ \\
\hline Asia centro-orientale e & 4 & 7 & 11 \\
sud-orientale & $36,4 \%$ & $63,6 \%$ & $100 \%$ \\
\hline Europa balcanica e orientale & 6 & 10 & 16 \\
& 37,8 & $62,2 \%$ & $100 \%$ \\
\hline Europa occidentale & 52 & 21 & 73 \\
& $71,2 \%$ & $28,8 \%$ & $100 \%$ \\
\hline Medio Oriente & 1 & 35 & 36 \\
\hline Nord Africa & $2,8 \%$ & $97,2 \%$ & $100 \%$ \\
\hline Nord America & 1 & 18 & 19 \\
\hline Totale & $5,3 \%$ & $94,7 \%$ & $100 \%$ \\
\hline
\end{tabular}

Il 73,9\% degli studenti intervistati di cittadinanza non italiana vive nel nostro Paese da oltre cinque anni (Tab. 5). Scorrendo in maniera più dettagliata tra i dati a disposizione è possibile affermare che coloro che vi risiedono da più tempo appartengono alla fascia dell'Europa balcanica e 
orientale (Albania, Romania, Macedonia, Ucraina, Polonia), dell'Europa occidentale (Grecia), del Nord Africa e dell'America centrale e meridionale, dati che coincidono abbastanza con le provenienze e i tempi di presenza media delle maggiori comunità, sull'intero territorio nazionale (IDOS, 2014). Non sempre, tuttavia, le collettività più consistenti di immigrati hanno il maggior numero di studenti, come attestano per esempio i casi del Marocco e delle Filippine.

Tab. 5 - Studenti con cittadinanza non italiana: da quanto tempo sei in Italia?

\begin{tabular}{|c|c|c|c|c|c|c|c|}
\hline $\begin{array}{l}\% \text { area geografica } \\
\text { di provenienza }\end{array}$ & $\begin{array}{c}\text { Da } \\
\text { meno di } \\
6 \text { mesi }\end{array}$ & $\begin{array}{l}\text { Da } 6 \text { a } \\
12 \text { mesi }\end{array}$ & $\begin{array}{l}\text { Da } 1 \text { a } \\
2 \text { anni }\end{array}$ & $\begin{array}{l}\text { Da } 2 \text { a } \\
5 \text { anni }\end{array}$ & $\begin{array}{l}\text { Da più } \\
\text { di } 5 \\
\text { anni }\end{array}$ & $\begin{array}{l}\text { Sono } \\
\text { nato/a } \\
\text { in Italia }\end{array}$ & Totale \\
\hline $\begin{array}{l}\text { Africa } \\
\text { subsahariana }\end{array}$ & $\begin{array}{r}4,5 \% \\
1\end{array}$ & $\begin{array}{r}4,5 \% \\
1\end{array}$ & $\begin{array}{r}22,7 \% \\
5\end{array}$ & $\begin{array}{r}22,7 \% \\
6\end{array}$ & $\begin{array}{r}45,5 \% \\
10\end{array}$ & $\begin{array}{r}0 \% \\
0\end{array}$ & $\begin{array}{r}100 \% \\
22\end{array}$ \\
\hline $\begin{array}{l}\text { America centrale } \\
\text { e meridionale }\end{array}$ & $\begin{array}{r}0 \% \\
0\end{array}$ & $\begin{array}{r}9,1 \% \\
1\end{array}$ & $\begin{array}{r}0 \% \\
0\end{array}$ & $\begin{array}{r}0 \% \\
0\end{array}$ & $\begin{array}{r}90,9 \% \\
10\end{array}$ & $\begin{array}{r}0 \% \\
0\end{array}$ & $\begin{array}{r}100 \% \\
11\end{array}$ \\
\hline $\begin{array}{l}\text { Asia centro- } \\
\text { orientale e sud- } \\
\text { orientale }\end{array}$ & $\begin{array}{r}6,3 \% \\
1\end{array}$ & $\begin{array}{r}18,8 \% \\
3\end{array}$ & $\begin{array}{r}25,0 \% \\
4\end{array}$ & $\begin{array}{r}0 \% \\
0\end{array}$ & $\begin{array}{r}50,0 \% \\
8\end{array}$ & $\begin{array}{r}0 \% \\
0\end{array}$ & $\begin{array}{r}100 \% \\
16\end{array}$ \\
\hline $\begin{array}{l}\text { Europa balcanica } \\
\text { e orientale }\end{array}$ & $\begin{array}{r}0 \% \\
0\end{array}$ & $\begin{array}{r}1,4 \% \\
1\end{array}$ & $\begin{array}{r}4,2 \% \\
3\end{array}$ & $\begin{array}{r}5,6 \% \\
4\end{array}$ & $\begin{array}{r}88,9 \% \\
64\end{array}$ & $\begin{array}{r}0 \% \\
0\end{array}$ & $\begin{array}{r}100 \% \\
72\end{array}$ \\
\hline $\begin{array}{l}\text { Europa } \\
\text { occidentale }\end{array}$ & $\begin{array}{r}0 \% \\
0\end{array}$ & $\begin{array}{r}2,8 \% \\
1\end{array}$ & $\begin{array}{r}5,6 \% \\
2\end{array}$ & $\begin{array}{r}11,1 \% \\
4\end{array}$ & $\begin{array}{r}80,6 \% \\
29\end{array}$ & $\begin{array}{r}0 \% \\
0\end{array}$ & $\begin{array}{r}100 \% \\
36\end{array}$ \\
\hline Medio Oriente & $\begin{array}{r}0 \% \\
0\end{array}$ & $\begin{array}{r}10,5 \% \\
2\end{array}$ & $\begin{array}{r}0 \% \\
0\end{array}$ & $\begin{array}{r}36,5 \% \\
7\end{array}$ & $\begin{array}{r}52,6 \% \\
10\end{array}$ & $\begin{array}{r}0 \% \\
0\end{array}$ & $\begin{array}{r}100 \% \\
19\end{array}$ \\
\hline Nord Africa & $\begin{array}{r}0 \% \\
0\end{array}$ & $\begin{array}{r}0 \% \\
0\end{array}$ & $\begin{array}{r}0 \% \\
0\end{array}$ & $\begin{array}{r}33,3 \% \\
2 \\
\end{array}$ & $\begin{array}{r}66,7 \% \\
4\end{array}$ & $\begin{array}{r}2,8 \% \\
1\end{array}$ & $\begin{array}{r}100 \% \\
6\end{array}$ \\
\hline Nord America & $\begin{array}{r}0 \% \\
0\end{array}$ & $\begin{array}{r}0 \% \\
0\end{array}$ & $\begin{array}{r}0 \% \\
0\end{array}$ & $\begin{array}{r}0 \% \\
0\end{array}$ & $\begin{array}{r}100 \% \\
1 \\
\end{array}$ & $\begin{array}{r}0 \% \\
0\end{array}$ & $\begin{array}{r}100 \% \\
1\end{array}$ \\
\hline Totale & $\begin{array}{r}1,1 \% \\
2\end{array}$ & $\begin{array}{r}4,9 \% \\
9\end{array}$ & $\begin{array}{r}7,6 \% \\
14\end{array}$ & $\begin{array}{r}12,0 \% \\
22\end{array}$ & $\begin{array}{r}73,9 \% \\
136\end{array}$ & & $\begin{array}{r}100 \% \\
184\end{array}$ \\
\hline
\end{tabular}

Gli studenti greci costituiscono una parte fondamentale del campione preso in esame, tuttavia notiamo come la Grecia, che storicamente è stato un Paese "esportatore" di studenti universitari grazie anche alla vicinanza geografica e dunque della facilità nei collegamenti, oggi vede una diminuzione generale della mobilità dei suoi studenti in Italia e, di conseguenza, anche verso l'ateneo aquilano (cfr. cap. 7). Il fenomeno potrebbe essere anche un riflesso del periodo di crisi e della riduzione degli incen- 
tivi che lo stato greco garantisce ai suoi cittadini per lo studio all'estero (IDOS, 2014).

La diminuzione della migrazione universitaria temporanea, tipica dunque degli iscritti ellenici, viene perlopiù compensata da quella di studenti provenienti in particolare dall'Albania e dalla Romania, con una diversa tendenza di base: se si considera che la percentuale maggiore di iscritti provenienti dalla regione dei Balcani ha nazionalità del proprio Paese di origine, ma vive con la famiglia in Italia, il carattere della $m i$ grazione universitaria della suddetta tipologia di studenti, appare molto meno temporaneo e molto più legato a spostamenti per ricongiungimento familiare.

L'aumento, all'interno dell'Università degli Studi dell'Aquila, degli iscritti provenienti da altre Nazioni dell'Unione Europea e, nella fattispecie, dalla Romania, è sostanzialmente in linea con ciò che emerge sia a livello regionale, sia a livello nazionale, dall'analisi dei dati ISTAT, ovvero si è assistito ad un incremento della collettività romena negli ultimi anni maggiore nel Centro Italia, dove il livello di istruzione delle comunità provenienti dall'Area UE è superiore rispetto al resto del territorio nazionale (Ibidem).

Gli studenti provenienti dalla regione subsahariana, dall'Asia centrale e orientale e dal Medio Oriente, risultano in media residenti in Italia da tempi più recenti (rispettivamente circa il $32 \%$, circa il $50 \%$ e quasi il $48 \%$ da meno di cinque anni).

In netta controtendenza rispetto a quanto emerge dai dati MIUR 2013/14, che mostrano un incremento degli alunni con cittadinanza non italiana ma nati in Italia nelle aule scolastiche del nostro Paese, nel campione dei 184 iscritti all'Univaq la percentuale degli aventi cittadinanza estera ma di nascita italiana risulta alquanto bassa. Solo l'1,7\% degli intervistati, infatti, è nato in Italia.

Il Paese dell'Africa equatoriale che conta più iscritti, stando alle rilevazioni, è il Camerun, fenomeno che rispecchia quanto avviene su scala nazionale. In base alle statistiche fornite dalla Caritas italiana (IDOS, 2014), dopo Francia, Inghilterra e Germania, l'Italia è il quarto Paese con il maggior numero di studenti camerunensi, agevolati anche dalla diffusione della lingua italiana in Camerun.

Confrontando i dati raccolti nel corso della nostra indagine emerge come la provenienza geografica influisca anche sul livello di reddito degli studenti. Il 41,2\% degli studenti internazionali e il 47,7\% degli studenti stranieri scolarizzati in Italia dichiarano di rientrare in una fascia di reddito bassa, a fronte del 13\% degli italiani che dichiarano di rientrare nella medesima categoria. Una percentuale più cospicua di intervistati, sostanzialmente analoga relativamente alle varie tipologie (28,9\% italiani, $29,4 \%$ 
studenti internazionali, 32,3\% stranieri), si colloca in una posizione più intermedia, tra il basso e il medio/basso profilo (Tab. 6). Si ritrova una differenza più marcata nelle fasce di reddito "media" (48,3\% degli italiani $v s$ il $22,5 \%$ dei cittadini non italiani, presi complessivamente) e "medio-alta/alta" (10\% circa di italiani, $4 \%$ circa di cittadini non italiani). Il valore del Chi Quadro restituisce il massimo livello di significatività statistica. È interessante notare come, anche all'interno dei due sottogruppi di studenti di cittadinanza non italiana, si osservi una lieve tendenza degli studenti internazionali a collocarsi su una posizione di relativo vantaggio rispetto agli studenti stranieri scolarizzati in Italia.

Tab. 6 - Condizione socio-economica degli studenti internazionali, stranieri scolarizzati in Italia, italiani

\begin{tabular}{lrrrrrr}
\hline & Bassa & $\begin{array}{c}\text { Medio/ } \\
\text { bassa }\end{array}$ & Media & $\begin{array}{c}\text { Medio/ } \\
\text { alta }\end{array}$ & Alta & Totale \\
\hline $\begin{array}{l}\text { Studenti } \\
\text { internazionali }\end{array}$ & $41,2 \%$ & $29,4 \%$ & $24,4 \%$ & $4,2 \%$ & $0,8 \%$ & $100,0 \%$ \\
\hline $\begin{array}{l}\text { Studenti } \\
\text { stranieri }\end{array}$ & 31 & 21 & 11 & 2 & 0 & 65 \\
scol. in It. & $47,7 \%$ & $32,3 \%$ & $16,9 \%$ & $3,1 \%$ & $0,0 \%$ & $100,0 \%$ \\
$\begin{array}{l}\text { Studenti } \\
\text { italiani }\end{array}$ & 51 & 115 & 192 & 35 & 5 & 398 \\
\hline Totale & $12,8 \%$ & $28,9 \%$ & $48,3 \%$ & $8,8 \%$ & $1,3 \%$ & $100,0 \%$ \\
\hline & $22,5 \%$ & $29,4 \%$ & $40,0 \%$ & $7,2 \%$ & $1,0 \%$ & $100,0 \%$ \\
\hline Chi Quadro di Pearson & Valore & Df & \multicolumn{3}{c}{ Sig. asint. (2 vie) } \\
\cline { 2 - 7 } & $81,798^{\text {a }}$ & 9 & \multicolumn{3}{c}{, 000} \\
\hline
\end{tabular}

L'indicatore di reddito sommato al fattore livello di istruzione dei genitori, per rappresentare il quale si è attribuito un punteggio sulla base del titolo di studio conseguito, ci dà un'indicazione orientativa sullo status socio-economico degli intervistati (Tab. 7). Gli studenti con una condizione reddituale più agevole sono in percentuale maggiore tra gli italiani e tra i non italiani di provenienza principalmente asiatica e mediorientale. Osservando, invece, le percentuali relative ai titoli di studio conseguiti da entrambi i genitori (Graf. 1) nei due campioni di intervistati, è possibile notare una predominanza di laureati (36,6\% maschi, 30,6\% femmine) e possessori di qualifiche professionali tra i genitori di cittadinanza non italiana, a fronte di una maggioranza di diplomati tra i genitori italiani $(41,6 \%$ maschi, 40,4\% femmine). 
Tab. 7 - Titolo di studio dei genitori

\begin{tabular}{lrrrr}
\hline & \multicolumn{2}{c}{ Studenti italiani } & \multicolumn{2}{c}{$\begin{array}{c}\text { Studenti di altra } \\
\text { cittadinanza }\end{array}$} \\
\hline & Padre & Madre & Padre & Madre \\
\hline Nessuno & $0,0 \%$ & $0,0 \%$ & $2,7 \%$ & $3,2 \%$ \\
\hline Scuola elementare & $5,8 \%$ & $7,3 \%$ & $4,8 \%$ & $5,4 \%$ \\
\hline Scuola media & $26,3 \%$ & $23,8 \%$ & $11,8 \%$ & $16,2 \%$ \\
\hline Qualifica professionale & $12,5 \%$ & $8,8 \%$ & $19,4 \%$ & $14,0 \%$ \\
\hline Scuola superiore & $40,4 \%$ & $41,6 \%$ & $24,7 \%$ & $30,6 \%$ \\
\hline Laurea & $15,0 \%$ & $18,5 \%$ & $36,6 \%$ & $30,6 \%$ \\
\hline
\end{tabular}

Graf. 1 - Titolo di studio genitori

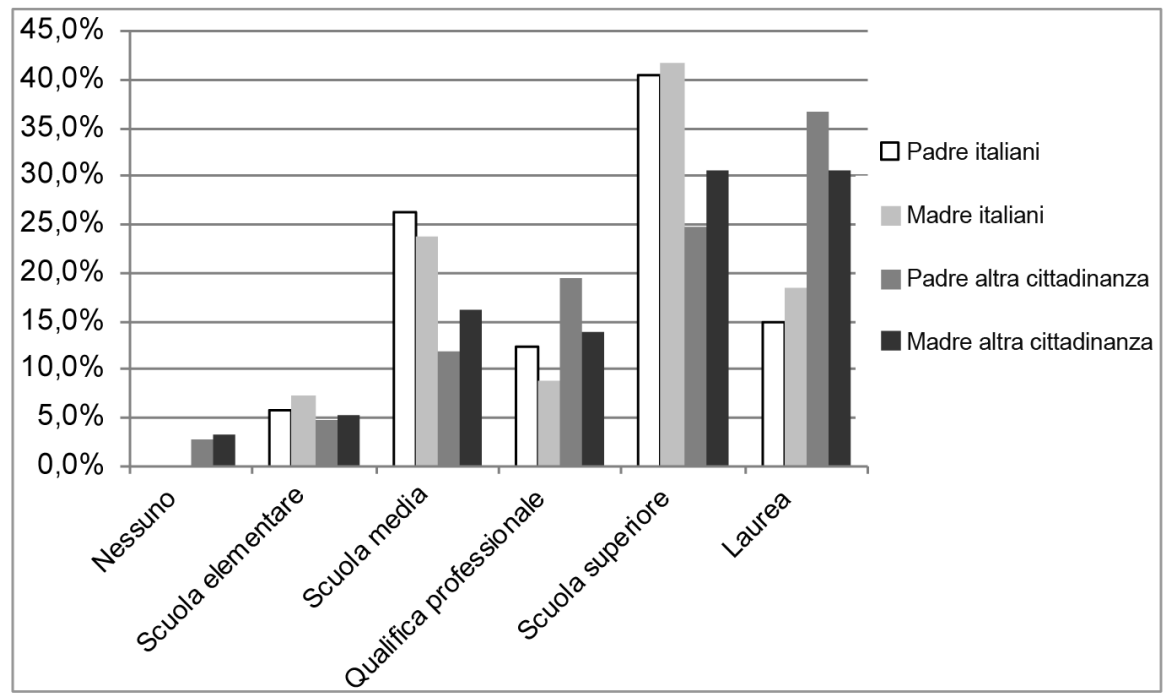

Attraverso l'applicazione del coefficiente non parametrico di correlazione rho di Spearman, abbiamo studiato le relazioni tra variabili all'interno dei tre sottogruppi individuati (studenti italiani, studenti internazionali, studenti stranieri scolarizzati in Italia), per verificare se esistono delle diverse intensità nei rapporti tra variabili (Tab. 8). Nella matrice abbiamo riportato esclusivamente le correlazioni con significatività di almeno 0,05 ; le correlazioni non significative sono state contrassegnate con un "no" al fine di rendere più leggibile la tabella. 
È interessante, in prima battuta, la correlazione di tipo negativo tra l'età da un lato e il titolo di studio della madre e lo status socio-economico, che osserviamo soltanto tra gli studenti italiani. In questo senso si può ravvisare tra i nostri connazionali una tendenza a studiare in età più adulta o al permanere più lungamente negli studi da parte di quei soggetti con una più bassa estrazione socioculturale che, al contrario, non si ravvisa tra gli studenti di altra cittadinanza. Un altro dato particolarmente interessante riguarda la relazione tra la fascia di reddito e il titolo di studio dei genitori che, mentre nel caso degli studenti italiani e di quelli internazionali si correlano positivamente in modo significativo, nel caso degli studenti stranieri scolarizzati in Italia non restituisce valori significativi. In questo caso si può rinviare alla condizione di quelle famiglie migranti per le quali la relazione tra titoli di studio e posizioni lavorative e reddituali non è così scontata e lineare. Questo lascia intendere che nel sottogruppo di studenti stranieri scolarizzati in Italia l'impatto del background culturale familiare non sempre è associato ad una adeguata condizione economica, che invece troviamo negli altri casi.

Tab. 8 - Età e background culturale ed economico: studenti italiani, internazionali, stranieri

\begin{tabular}{llrrrrr}
\hline & & Età & $\begin{array}{r}\text { Titolo } \\
\text { studio } \\
\text { madre }\end{array}$ & $\begin{array}{r}\text { Titolo } \\
\text { studio } \\
\text { padre }\end{array}$ & $\begin{array}{r}\text { Fascia } \\
\text { reddito }\end{array}$ & $\begin{array}{l}\text { Indice } \\
\text { Status }\end{array}$ \\
\hline Età & Italiani & 1 & & & & \\
& Stranieri & 1 & & & & \\
& Internazionali & 1 & & & & \\
\hline Titolo studio & Italiani & $-0,249$ & 1 & & & \\
madre & Stranieri & no & 1 & & & \\
& Internazionali & no & 1 & & & \\
\hline Titolo studio & Italiani & no & 0,542 & 1 & & \\
padre & Stranieri & no & 0,537 & 1 & & \\
& Internazionali & no & 0,667 & 1 & & \\
\hline Fascia reddito & Italiani & no & 0,308 & 0,372 & 1 & \\
& Stranieri & no & no & no & 1 & \\
& Internazionali & no & 0,225 & 0,152 & & 1 \\
\hline Indice Status & Italiani & $-0,222$ & 0,821 & 0,840 & 0,639 & 1 \\
& Stranieri & no & 0,763 & 0,856 & 0,451 & 1 \\
& Internazionali & no & 0,877 & 0,838 & 0,502 & 1 \\
\hline
\end{tabular}


Calcolando la media dell'Indice di Status per ogni macro-area geografica di appartenenza, appare evidente che le aree più simili tra loro risultano essere l'Italia, l'Europa occidentale e l'Europa balcanica (con un indice medio di 11.1); l'Asia, l'America centrale e meridionale e il Medio Oriente (con un indice medio di 10.2) ed infine l'Africa subsahariana e il Nord Africa (con un indice medio di 9.5).

Laddove il reddito risulta più basso, spesso interviene una sorta di compensazione a livello di istruzione familiare; ciò riesce a colmare il dislivello creato dalla condizione socio-economica, nonostante le differenze sostanziali che in termini sociali ed economici si possono riscontrare mettendo a confronto i diversi luoghi di provenienza.

Al quesito "Chi paga i tuoi studi?" (Graf. 2) la maggior parte degli studenti italiani (57\%) dichiara di essere dedita principalmente allo studio e che le spese per l'istruzione sono a carico dei familiari, a fronte del $40 \%$ degli studenti di cittadinanza non italiana. Tra questi ultimi, la differenza percentuale tra gli studenti internazionali e quelli stranieri scolarizzati in Italia è di 4,7 punti (37,8\% studenti internazionali - 43,1\% stranieri). Tale divario sembra compensarsi attraverso l'accesso a borse di studio, che nel primo caso pesa per il $12,6 \%$ e nel secondo per il $6,2 \%$.

Graf. 2 - Chi paga i tuoi studi?

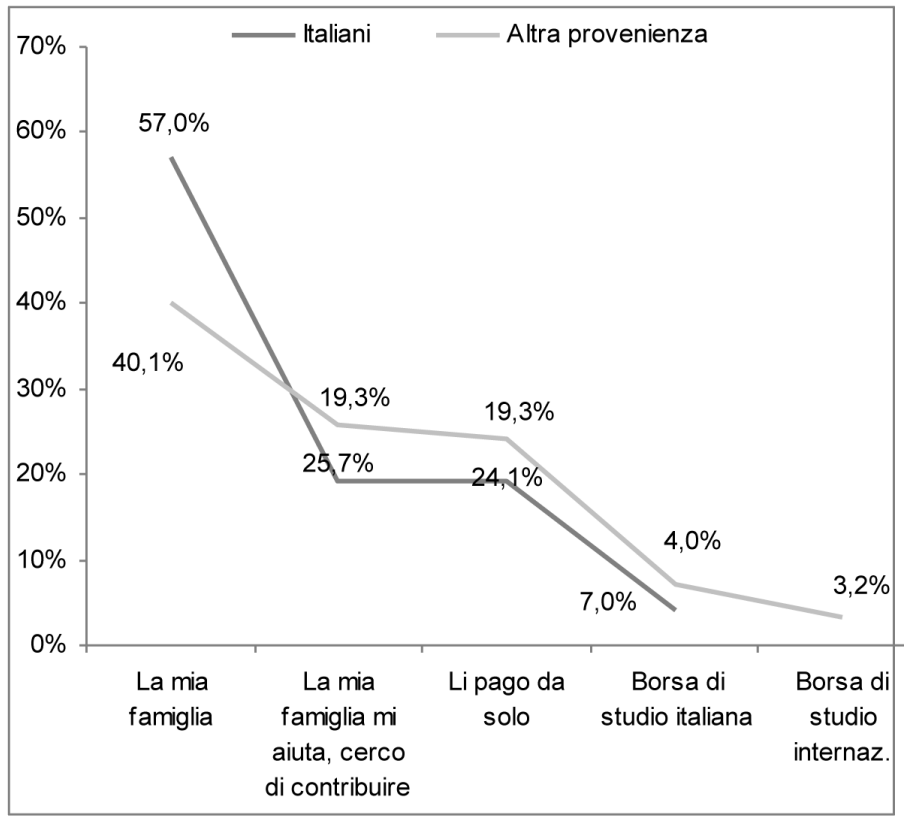


Approfondendo l'analisi per provenienza geografica emerge come, tra gli intervistati provenienti sia dall'Europa occidentale sia dal Medio Oriente, la percentuale di coloro che sono totalmente a carico dei familiari superi il $63 \%$, mentre al contrario, il $54 \%$ dei sudamericani dichiara di provvedere personalmente alla propria istruzione universitaria. Tra gli intervistati dell'Africa subsahariana, soltanto 3 su 22 sono gli studenti totalmente sostenuti dalla famiglia $(13,6 \%)$. All'interno dello stesso gruppo, riscontriamo il maggior numero di beneficiari di borse di studio (6 su 22).

Dei 185 studenti di cittadinanza non italiana che hanno risposto al questionario, il 44,4\% possiede un permesso di soggiorno per motivi di studio, il $26,7 \%$ è titolare di una carta di soggiorno ${ }^{3}$ e il $10 \%$ circa ha ottenuto un permesso per motivi di ricongiungimento familiare (Graf. 3). Si registra un solo caso di rilascio di permesso di soggiorno per asilo politico (uno studente proveniente dal Nord Africa). Il 15,5\% degli intervistati non ha permesso di soggiorno e il $2,7 \%$ possiede un permesso scaduto e non rinnovato.

\section{Graf. 3 - Il permesso di soggiorno}

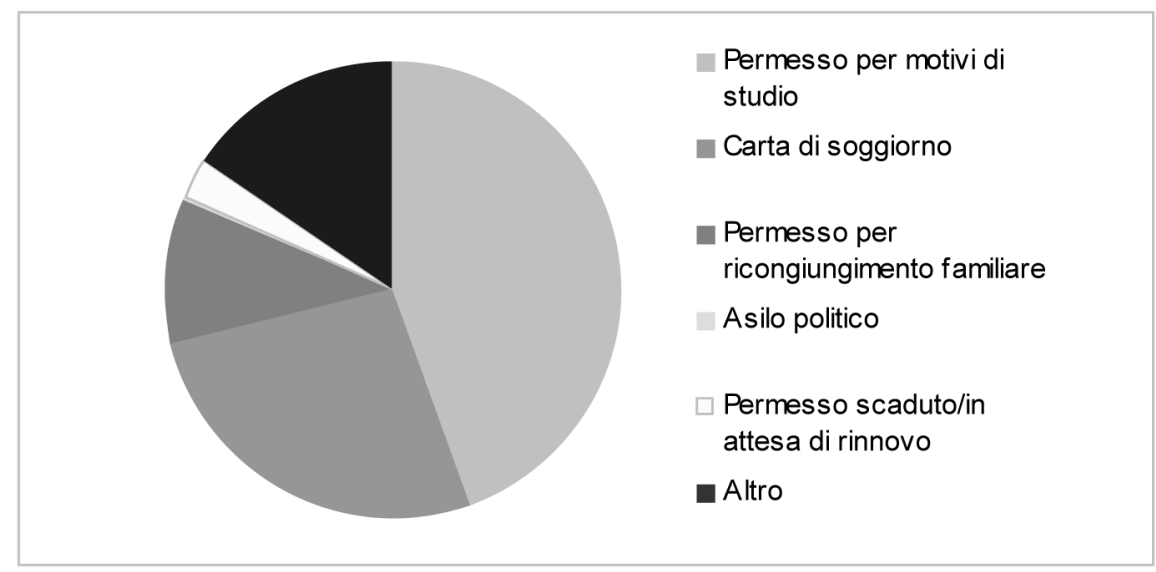

3. Dal $1^{\circ}$ aprile 2007 , data di entrata in vigore del d.lgs. 30/2007, la denominazione "carta di soggiorno" si utilizza soltanto per il titolo di soggiorno rilasciato agli stranieri extra-comunitari familiari di cittadini comunitari (e italiani) residenti sul territorio italiano, in possesso dei requisiti richiesti, ai sensi dell'art. 10 del d.lgs. 6.02.2007, n. 30, a cui viene rilasciata la "Carta di soggiorno per familiare di cittadino comunitario". Il permesso di soggiorno CE di lungo periodo, ai sensi d.lgs. 286/98, può essere richiesto dallo straniero extracomunitario in possesso, da almeno cinque anni, di un permesso di soggiorno in corso di validità, che dimostri la disponibilità di un reddito minimo non inferiore all'importo annuo dell'assegno sociale e di un alloggio idoneo e che non sia pericoloso per l'ordine pubblico o la sicurezza dello Stato. Il rilascio è subordinato al superamento del test di conoscenza della lingua italiana. 
Più dettagliatamente: gli intervistati che possiedono un permesso di soggiorno (prevalentemente o esclusivamente) per motivi di studio, oppure una carta di soggiorno, sono studenti provenienti dall'Africa subsahariana (81,8\% PS; 9\% CS), dal Medio Oriente (78,9\% PS; 15,8\% CS), dall'America centrale e meridionale (36,5\% PS; $45,5 \%$ CS) e dal Nord Africa $(50 \%$ PS; $15 \% \mathrm{CS}$ ). La più alta concentrazione di permessi scaduti/non rinnovati si rileva tra i cittadini dell'area dell'Asia centro-orientale e sud-orientale (3 su 16). Riguardo ai cittadini comunitari, la disciplina è la seguente: «Tutti i cittadini dell'Unione Europea hanno il diritto di entrare e soggiornare liberamente in Italia o in un altro Stato membro diverso da quello di cui hanno la cittadinanza, con modalità differenti a seconda che il periodo di soggiorno sia di durata inferiore o superiore a tre mesi». Agli studenti provenienti dall'Europa occidentale e dall'Europa balcanica e orientale, anche quando cittadini comunitari, è dunque richiesto il permesso di soggiorno per motivi di studio; il 25\% di essi, però, dichiara di non averne o di non averne bisogno in quanto cittadino comunitario, italiano, oppure perché in attesa del riconoscimento della cittadinanza italiana.

Gli intervistati originari dell'area balcanica e orientale, in particolare, sono titolari di permessi di soggiorno per ricongiungimento familiare in percentuale più alta $(22,2 \%$, ovvero 16 soggetti su 62$)$ rispetto ai cittadini di altre aree. Stando a quanto emerge da una recente analisi sulle ragioni del soggiorno (IDOS, 2014), nell'anno 2013 la quota di titoli emessi per motivi di famiglia è in crescita rispetto ai permessi rilasciati per altre motivazioni, compresi quelli per studio, che rappresentano il $10.7 \%$ del totale. Questo dato è associabile alla tendenziale stabilità e al radicamento a carattere familiare di ampi strati di popolazione non comunitaria (e, negli ultimi anni, anche comunitaria), soggiornante nel nostro Paese.

Uno scenario diversificato, dunque, quello legato alla mobilità studentesca non autoctona, che assume diversi aspetti, in base alle condizioni socio-economiche dei Paesi e delle famiglie di provenienza e alle motivazioni che portano un giovane a decidere di studiare all'estero o di proseguire gli studi nel Paese in cui vive. Un contesto parallelo all'interno del quale convive chi può godere di una maggiore sicurezza economica e chi invece si trova in uno stato più precario; chi si muove all'interno di un'area culturale condivisa e chi invece proviene da tradizioni più distanti; chi è spinto dal desiderio di inserirsi nel Paese che l'accoglie e chi invece assapora un'esperienza temporanea. 


\title{
9. La formazione scolastica e universitaria: i percorsi, gli esiti, la motivazione allo studio
}

\author{
di Valentina Ciaccio
}

\subsection{La formazione scolastica nei due campioni}

Secondo il Dossier di Tuttoscuola "Dispersione nella scuola secondaria superiore" (giugno 2013) basato sull'elaborazione di dati MIUR relativi agli ultimi 15 anni, il 31,9\% degli studenti abbandona il percorso formativo di II grado senza aver conseguito il diploma. Tale percentuale, che in alcune regioni (fra cui le Isole) arriva a quota $36 \%$, vede al suo interno una rilevante fetta di studenti di cittadinanza non italiana. Gli alunni stranieri ${ }^{1}$, nell'a.s. 2013/14, rappresentavano il 9\% del totale; di questi il 51,7\% è nato in Italia, percentuale che passa dall' $84 \%$ nelle scuole dell'infanzia al $20,9 \%$ di iscritti al primo anno della secondaria di II grado per arrivare al $10,1 \%$ di nati in Italia presenti nella classe quinta della scuola secondaria di II grado (MIUR e ISMU, 2015). Secondo uno studio MIUR sul rischio di abbandono durante l'anno scolastico ${ }^{2}$ tali studenti, iscritti nelle scuole superiori di II grado, sono a rischio di abbandono durante l'anno scolastico nel $2,42 \%$ dei casi, contro l'1,16\% degli italiani. Inoltre, emerge una situazione di maggiore difficoltà per gli alunni nati all'estero rispetto a quelli di seconda generazione: degli alunni stranieri che non terminano la scuola di II grado, il 92\% è composto da stranieri di prima generazione (MIUR, 2013b). Tale dato è probabilmente indicativo di quanto la conoscenza della lingua e l'integrazione sociale possano supportare il percorso formativo.

Per avere maggiori informazioni riguardo l'esperienza scolastica dei due campioni di studenti universitari, abbiamo posto loro alcune domande riguardanti la scelta di percorso superiore di II grado, l'esito e la percezione

1. Prendendo in considerazione tutti i livelli di istruzione scolastica.

2. I dati rilevati riguardano l'abbandono durante l'anno scolastico e non prendono in considerazione le interruzioni di frequenza nel passaggio da un anno di corso a quello successivo. 
di efficacia della scuola italiana. All'interno del nostro studio non si notano differenze significative, fra gli studenti italiani e quelli con cittadinanza non italiana, per quanto concerne la scelta del percorso di studi superiori (Chi Quadro con Sig. ,000): in entrambi i gruppi circa il 66\% ha frequentato un Liceo ad indirizzo umanistico o scientifico, mentre circa il 34\% un Istituto tecnico o un Istituto professionale (scarto percentuale dello 0,9\%). A livello nazionale, invece, nell'a.s. 2013/14 il 76,4\% degli alunni stranieri risultava iscritta ad istituti tecnici e professionali, contro il 52,3\% degli italiani (MIUR e ISMU, 2014).

Analizzando invece il voto di diploma (Graf. 1) possiamo osservare le prime significative differenze fra i due gruppi, con un rendimento in uscita migliore per gli studenti con cittadinanza non italiana rispetto a quello degli italiani. Considerato che quest'ultimo gruppo era composto sia da studenti che avevano almeno in parte frequentato e concluso il periodo di scolarizzazione in Italia, sia da studenti che avevano conseguito il titolo nel Paese d'origine, abbiamo esaminato separatamente i due sottogruppi ${ }^{3}$. Come osserviamo nel Grafico 1 (Chi Quadro con Sig. ,000) abbiamo un picco del $43 \%$ di studenti stranieri che hanno frequentato la scuola in Italia, il cui voto finale si attesta nella fascia media 71-80, e una percentuale del

Graf. 1 - Fasce di voto di diploma - Studenti italiani, stranieri scolarizzati in Italia, internazionali

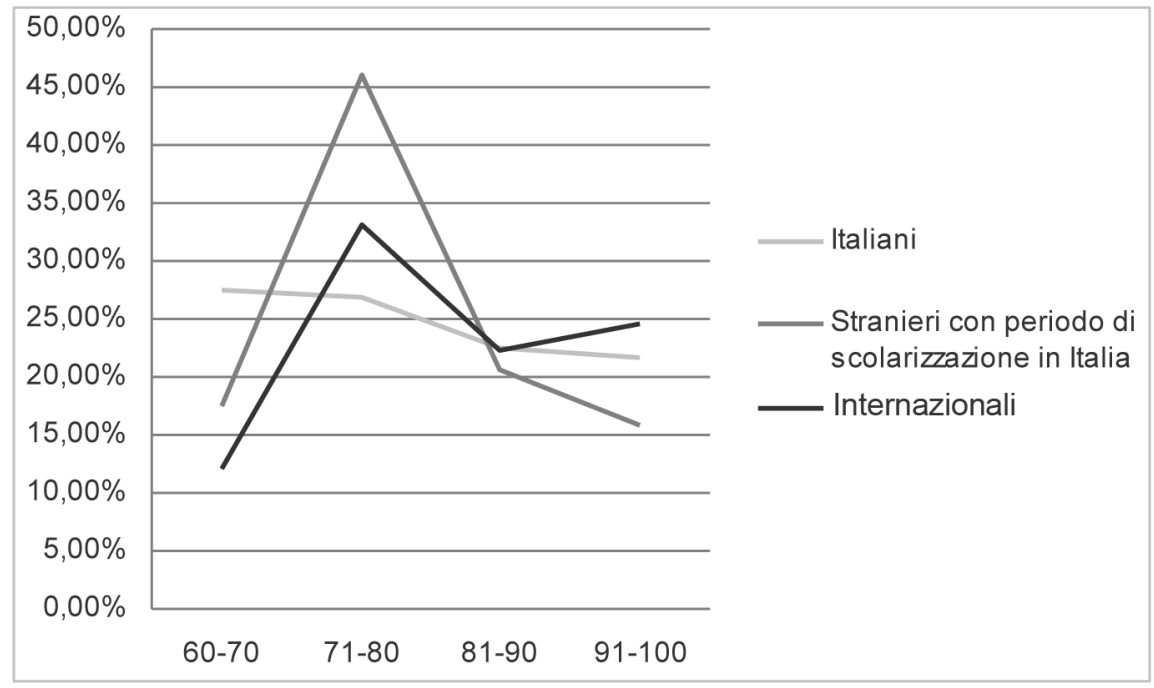

3. Il campione degli studenti stranieri nati in Italia era troppo esiguo per essere preso in considerazione come categoria a se stante. 
$20,6 \%$, di poco inferiore a quella degli italiani $(22,6 \%)$, nella fascia medio/ alta 81-90. Confrontando il dato degli studenti internazionali con quello degli italiani, osserviamo anche in questo caso un rendimento in uscita migliore nel primo gruppo: il 30,6\% degli studenti con titolo conseguito all'estero si attesta nella fascia alta di votazione, $91-100$, contro il $21,60 \%$ relativo al dato del secondo campione.

Va precisato che gli studi comparativi del rendimento in uscita degli italiani rispetto agli studenti di cittadinanza non italiana, a livello nazionale, mostrano una scarsa presenza di questi ultimi nelle fasce di rendimento eccellente ed una maggiore concentrazione nelle fasce di voto più basse (MIUR e ISMU, 2014).

I dati del nostro campione appaiono ancor più sorprendenti prendendo in considerazione il livello di conoscenza della lingua prima di arrivare in Italia: 1'81\% degli stranieri che ha frequentato almeno in parte la scuola italiana non aveva nessuna conoscenza della lingua italiana, mentre il 9,5\% aveva solo una minima conoscenza della stessa. Inoltre, soltanto il $24 \%$ di essi ha frequentato un corso di lingua italiana.

Oltre alla conoscenza della lingua, quali altri elementi possono influenzare il percorso formativo degli alunni? Vari studi hanno mostrato gli effetti positivi delle buone relazioni interpersonali sulla motivazione e sul rendimento scolastico: il clima sociale è influenzato dal coinvolgimento e può essere predittivo della prestazione. Abbiamo quindi voluto comprendere quale fosse, nei due campioni, la percezione della capacità della scuola italiana di supportare gli alunni di cittadinanza non italiana, prendendo in considerazione diversi ambiti legati sia alla formazione dello studente sia alla sua integrazione sociale (Graf. 2). Gli indicatori sono stati rilevati in scala da 1 a 4 e comprendono le seguenti voci: lingua italiana; lingue straniere; apprendimenti nelle discipline umanistiche; apprendimenti nelle discipline tecnico-scientifiche; attività artistiche e/o sportive; integrazione sociale e benessere scolastico. Il punteggio medio, calcolato sull'indice sintetico riferito all'efficacia della scuola italiana nei confronti degli studenti di cittadinanza diversa (min. teorico 6 - max teorico 24) si attesta su un valore pari a 15,9 (deviazione std. 2,92) calcolato sul campione italiano, e di 16,6 (deviazione std. 3,13) sul sottogruppo degli studenti stranieri scolarizzati in Italia. La differenza media, che lascerebbe presupporre una valutazione più positiva da parte dei ragazzi stranieri, non è statisticamente significativa (Anova con Sig. 0,443) .

4. In entrambi i casi, l'efficacia della scuola italiana viene valutata su un livello sostanzialmente "medio". 
Graf. 2 - La percezione della capacità della scuola italiana di supportare i ragazzi stranieri nei diversi ambiti: studenti italiani e stranieri scolarizzati in Italia

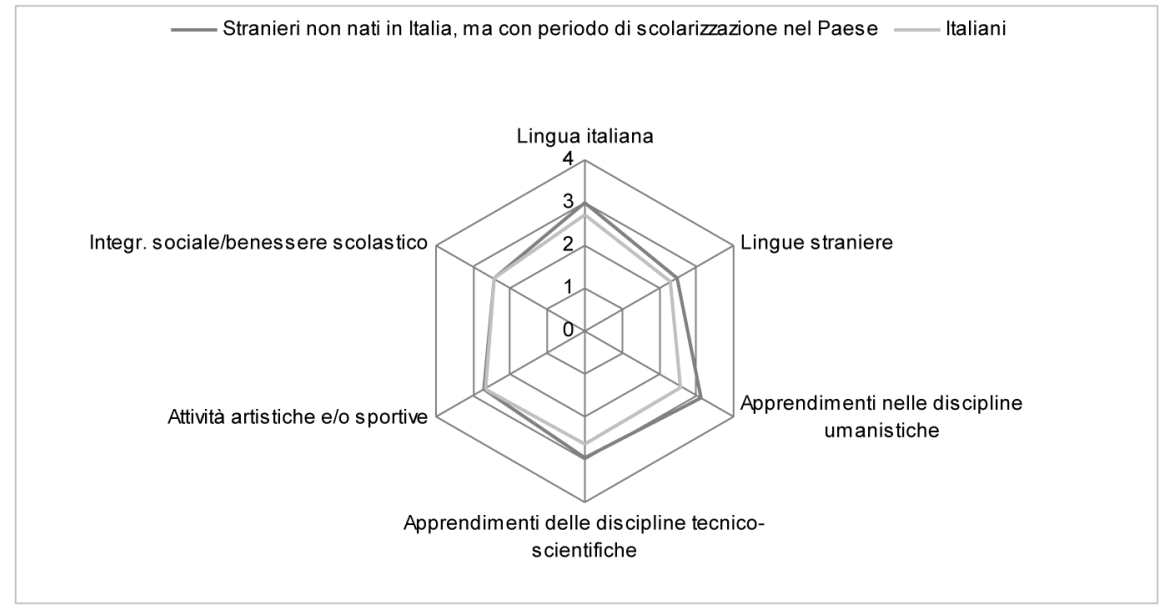

I valori divisi per le diverse aree, che prendendo in considerazione le differenze fra i due campioni sono quasi sovrapponibili, hanno valori leggermente più alti per gli studenti stranieri non nati in Italia, ma con un periodo di scolarizzazione nel Paese 5 .

Possiamo notare dei valori più elevati nell'insegnamento della lingua italiana e in quello delle discipline tecnico-scientifiche ed umanistiche, mentre compaiono valori più bassi nell'insegnamento della lingua straniera e nell'integrazione sociale e benessere scolastico. Quest'ultimo indicatore, che per gli studenti italiani raggiunge un valore medio di 2,42 (deviazione std. 0,721) e per gli studenti stranieri nati all'estero e con periodo di scolarizzazione in Italia raggiunge un valore medio di 2,43 (deviazione std. 0,875), meriterebbe un maggior approfondimento in un successivo studio per comprendere se e quali strumenti formativi vengono messi a disposizione degli insegnanti per migliorare il livello di integrazione sociale degli alunni stranieri e come tale variabile influisce nell'esito del loro percorso formativo. Precisiamo però che anche in questo caso, nei quesiti relativi all'insegnamento di lingua italiana, lingua straniera e nell'integrazione sociale e benessere scolastico, la differenza media non è statisticamente significativa (Anova con sig. rispettivamente di 0,156, 0,245 e 0,848) men-

5. Come nel caso del Grafico 1 sui voti di diploma, anche in questo caso il campione degli studenti stranieri nati in Italia era troppo esiguo per essere preso in considerazione come categoria a se stante. 
tre lo è per i quesiti relativi all'apprendimento nelle discipline umanistiche, tecnico-scientifiche e sportive (Anova con sig. rispettivamente di 0,011, $0,001$ e 0,061$)$.

Il quadro che ci si mostra davanti è quindi quello di una popolazione straniera che, nel caso non faccia parte della seconda generazione, è arrivata fra i banchi della scuola italiana con una quasi inesistente conoscenza della lingua, carenza che solo in 1 caso su 4 è stata colmata attraverso dei corsi appositi. Ciò probabilmente influisce significativamente sia sul rischio di abbandono durante l'anno scolastico, la cui percentuale è doppia per gli studenti stranieri rispetto agli italiani, sia sul senso di autoefficacia (Bandura, 2012) e quindi sull'esito del percorso formativo di quelli stranieri che, nonostante gli ostacoli, riescono non solo a terminare il percorso formativo di II grado, ma anche a farlo con un voto di diploma migliore di quello dei colleghi italiani.

\subsection{La scelta del percorso universitario}

Secondo il rapporto dell'OECD sull'istruzione intitolato Education at a Glance 2014: OECD Indicators (OECD, 2014), gli studenti di diversa nazionalità sono portati a scegliere diversi corsi di studi rispetto agli studenti italiani: questo indica sia la presenza fra i corsi offerti dallo Stato non di origine di percorsi più specializzanti, sia un'insufficienza di corsi nei Paesi d'origine e/o migliori opportunità di impiego collegate con i specifici campi formativi ${ }^{6}$.

Nel quadro successivo (Tab. 1), nel quale viene riportata la distribuzione degli studenti nei 7 dipartimenti dell'ateneo, osserviamo notevoli differenze fra i due campioni presi in esame, statisticamente significative (Chi Quadro di Pearson con Sig. 0,000).

Nel Dipartimento Medicina clinica, sanità pubblica, scienze della vita e dell'ambiente (MESVA), che da solo contiene quasi metà dell'offerta formativa e della popolazione studentesca dell'ateneo, notiamo una differenza di ben 14,2 punti percentuali fra i due campioni, nello specifico, più della metà degli studenti con cittadinanza non italiana coinvolti nell'indagine è iscritta a tale dipartimento. Questo dato, oltre ad essere legato alle motivazioni elencate dallo studio OECD, è legato anche al contingente riservato agli studenti non comunitari, che è una quota di posti resi disponibili per tale categoria e comunicati attraverso i bandi d'accesso ai corsi di studi, emanati ogni anno accademico dagli atenei. Sulla base delle

6. Indicatore A4 all'interno del rapporto OECD (2011). 
Tab. 1 - La distribuzione degli studenti nei dipartimenti

\begin{tabular}{lcc}
\hline Dipartimento & $\begin{array}{c}\text { Studenti } \\
\text { italiani }\end{array}$ & $\begin{array}{c}\text { Studenti } \\
\text { con cittadinanza } \\
\text { non italiana }\end{array}$ \\
\hline $\begin{array}{l}\text { Medicina clinica, sanità pubblica, } \\
\text { scienze della vita e dell'ambiente (MESVA) }\end{array}$ & $38,6 \%$ & $52,8 \%$ \\
\hline $\begin{array}{l}\text { Ingegneria industriale e dell'informazione } \\
\text { e di economia (DIIIE) }\end{array}$ & $21,8 \%$ & $13,7 \%$ \\
\hline Scienze umane (DSU) & $18,5 \%$ & $13,0 \%$ \\
\hline $\begin{array}{l}\text { Ingegneria e scienze dell'informazione } \\
\text { e matematica (DISIM) }\end{array}$ & $7,3 \%$ & $8,7 \%$ \\
\hline $\begin{array}{l}\text { Ingegneria civile, edile-architettura, } \\
\text { ambientale (DICEAA) }\end{array}$ & $6,5 \%$ & $8,1 \%$ \\
\hline $\begin{array}{l}\text { Scienze cliniche applicate e } \\
\text { biotecnologiche (DISCAB) }\end{array}$ & $5,5 \%$ & $1,9 \%$ \\
\hline \begin{tabular}{l} 
Scienze fisiche e chimiche (DSFC) \\
\hline
\end{tabular} & $1,8 \%$ & $1,9 \%$ \\
\hline
\end{tabular}

disponibilità comunicate dalle università, con decreto del Ministro degli Affari Esteri, di concerto con il MIUR e con il Ministro dell'interno, è disciplinato annualmente il numero massimo dei visti di ingresso e dei permessi di soggiorno per l'accesso all'istruzione universitaria degli studenti residenti all'estero ${ }^{7}$. La rilevanza di ciò si può evincere anche dalla percentuale di studenti internazionali iscritti a corsi ad accesso programmato $(65,7 \%)$ rispetto agli italiani $(22,9 \%)$ e dal fatto che il Dipartimento MESVA contenga al suo interno quasi tutti i corsi a numero programmato dell'ateneo. Va precisato che non tutti gli studenti stranieri accedono ai corsi ad accesso programmato attraverso il contingente riservato, anche perché parte del campione di studenti con cittadinanza non italiana proviene da Stati dell'UE e/o ha conseguito il titolo di diploma in Italia (cfr. par. 3). Probabilmente anche tale fattore va ad influire nella scelta del corso di studi, che negli studenti stranieri scolarizzati in Italia ha un andamento più vicino a quello degli italiani rispetto a quello degli studenti internazionali (Tab. 2).

7. Art. 39 del d.lgs. del 25 luglio 1998, n. 268 "Testo unico delle disposizioni concernenti la disciplina dell'immigrazione e norme sulla condizione dello straniero". 


\begin{tabular}{lccc}
\hline & $\begin{array}{c}\text { Studenti } \\
\text { internazionali }\end{array}$ & $\begin{array}{c}\text { Studenti stranieri } \\
\text { scolarizzati in Italia }\end{array}$ & $\begin{array}{c}\text { Studenti } \\
\text { italiani }\end{array}$ \\
\hline $\begin{array}{l}\text { Iscrizione a corso con } \\
\text { numero programmato }\end{array}$ & $65,7 \%$ & $36,7 \%$ & $22,9 \%$ \\
\hline $\begin{array}{l}\text { Iscrizione a corso ad } \\
\text { accesso libero }\end{array}$ & $34,3 \%$ & $63,3 \%$ & $77,1 \%$ \\
\hline
\end{tabular}

(Chi Quadro con Sig. ,000)

Andando ad analizzare quali sono le aree geografiche maggiormente rappresentate all'interno della popolazione di studenti con cittadinanza non italiana iscritti ad un corso ad accesso programmato (sottogruppo composto da 88 studenti), abbiamo il 35,2\% proveniente da uno Stato dell'Unione Europea, il 18,2\% dall'Europa balcanica e orientale, il 14,8\% dall'Africa subsahariana e il 13,6\% dal Medio Oriente. Prendendo invece in considerazione, all'interno delle singole aree geografiche, la percentuale di iscritti a corsi ad accesso programmato, possiamo osservare le percentuali più alte fra gli studenti provenienti dal Medio Oriente (80\%), Asia centro-orientale e sud-orientale $(66,6 \%)$, Africa subsahariana $(61,9 \%)$ e Nord Africa 50\% (Chi Quadro con Sig. ,000).

Fra i corsi ad accesso programmato, nel campione degli studenti con cittadinanza non italiana, abbiamo una prevalenza di iscritti a Medicina e Chirurgia (31 di cui 26 internazionali), Infermieristica (9 di cui 4 internazionali) ed Odontoiatria e Protesi Dentaria (9 internazionali). La scelta di tali corsi di studi, caratterizzati da un'elevata percentuale di occupati fra i relativi laureati ${ }^{8}$ fa pensare ad una scelta degli stessi come investimento per il futuro, specialmente considerate le spese onerose a cui lo studente va incontro durante il suo percorso formativo". Dallo studio "Gli studenti internazionali nelle università italiane: indagine empirica e approfondimenti" (Ministero dell'Interno e IDOS, 2013) emerge come, per la maggioranza degli studenti non comunitari, venire in Italia per motivi di studio rappresenti una scelta in grado di offrire buone prospettive di carriera, sia in Italia che nel Paese di origine.

8. AlmaLaurea - Consorzio Universitario, XVI Indagine (2013) - Condizione occupazionale dei Laureati, 2014.

9. I dati OECD relativi al 2011 (Indicatore B5, OECD 2011) mostrano come l'Italia è la terza Nazione europea nella graduatoria dei Paesi con le tasse universitarie più elevate. Si calcola un ammontare di tasse universitarie, pagate dagli iscritti con cittadinanza non italiana, di $71 \mathrm{mln}$ di euro. In questo modo si calcola una spesa complessiva sostenuta da essi di 718,5mln di euro (EMN-Italia, 2012). 


\subsection{Requisiti di accesso richiesti e corsi integrativi ero- gati}

Rispetto al livello di studio richiesto all'ingresso e alla possibilità di accesso ai corsi di lingua italiani, vi è netta differenza fra norme ed opportunità relative agli studenti di cittadinanza non italiana, in mobilità, internazionali con cittadinanza comunitaria e non comunitaria ${ }^{10}$. L'ammissione degli studenti con cittadinanza non italiana negli atenei è regolata da normativa nazionale, con particolare riferimento all'art. 39 del d.lgs. n. 268 del 25 luglio 1998 "Testo unico delle disposizioni concernenti la disciplina dell'immigrazione e norme sulla condizione dello straniero". Essa viene regolamentata, in base alle varie casistiche, come segue:

- i candidati dell'Unione Europea ovunque residenti e quelli non dell'Unione Europea regolarmente soggiornanti in Italia $^{11}$, accedono senza limitazioni di contingente e senza una prova idoneativa di lingua italiana. I cittadini di Norvegia, Islanda, Lichtenstein, Svizzera e San Marino sono equiparati ai cittadini comunitari ai fini dell'accesso ai corsi delle università;

- i candidati non provenienti dall'Unione Europea o dagli Stati sopra menzionati devono partecipare ad una prova idoneativa di lingua italiana. Essi rientrano nel contingente a loro riservato e, se non classificati in graduatoria in posizione utile rispetto al numero dei posti loro riservati possono presentare una sola domanda di ammissione ad altro corso universitario presso la stessa sede o di ri-assegnazione, per lo stesso corso universitario o per altro, ad altra sede. Alcune categorie sono equiparate a quella degli studenti con cittadinanza italiana ai fini dell'accesso ai corsi delle università. Fra esse troviamo i rifugiati e i titolari di protezione sussidiaria, i titolari dei diplomi finali rilasciati dalle scuole italiane statali e paritarie all'estero e i cittadini stranieri in possesso di permesso di soggiorno UE per soggiornanti di lungo periodo.

La conoscenza della lingua italiana, per gli studenti con cittadinanza non comunitaria, viene verificata dalle Rappresentanze diplomatiche italiane nei Paesi di origine. Alternativamente, presso la sede universitaria scelta da ciascun candidato viene svolta una prova della conoscenza della lingua, obbligatoria per tutti i corsi universitari, verifica da cui si viene esonerati se si è in possesso di una certificazione di competenza di lingua italiana di livello C1, C2 o di livello non inferiore al B2 del Consiglio d'Europa, o in possesso del diploma di istruzione secondaria di II grado di durata quin-

10. Fonti per il paragrafo: MIUR, www.studiare-in-italia.it in riferimento alla normativa nazionale; www.univaq.it per quanto concerne i regolamenti locali.

11. Cfr. art. 39, comma 5, del d.lgs. n. 286 del 25.07.98, come modificato dall'art. 26 della L. 189 del 30.07.2002, "Modifica alla normativa in materia di immigrazione e di asilo". 
quennale o quadriennale solo se conseguito presso le scuole italiane statali e paritarie all'estero. Sono esonerati dalla suddetta verifica effettuata dalle Rappresentanze diplomatiche anche gli studenti che chiedono l'iscrizione ai corsi di studio erogati in lingua inglese, fra cui ad esempio il corso "MathMod", erogato dal Dipartimento di Ingegneria e Scienze dell'Informazione e Matematica (DISIM) dell'Università degli Studi dell'Aquila. Per quanto riguarda il livello di conoscenza della lingua in ingresso per gli studenti in mobilità, nei singoli accordi che vengono siglati sono indicati i livelli linguistici richiesti sia per l'inglese sia per l'italiano. Nonostante tale indicazione, durante diverse consultazioni col corpo docente riguardo alla tematica, è emersa una situazione di scarsa padronanza della lingua da parte degli studenti in mobilità in ingresso, fattore che spesso pregiudica la comprensione delle lezioni svolte in italiano.

Nell'Università degli Studi dell'Aquila gli studenti con cittadinanza non italiana, indipendentemente se iscritti o in mobilità, hanno a disposizione $\mathrm{i}$ moduli di Lingua e Cultura Italiana per stranieri, attivi per i livelli A1, A2, B1 e B2 ${ }^{12}$ del Quadro europeo per le lingue. Si tratta di moduli da 3 CFU, corrispondenti a 30-40 ore, che prevedono un esame finale scritto e orale nel corso del quale si verificano le competenze previste dal Quadro europeo. Tali corsi, facoltativi, sono codificati come tutti gli altri insegnamenti, per cui necessitano dell'inserimento nel piano di studi entro le scadenze amministrative previste dall'ateneo e dell'approvazione da parte del Consiglio di area didattica. Considerato tale iter burocratico, essi vengono frequentati maggiormente dagli studenti iscritti all'ateneo, rispetto agli studenti in mobilità.

\subsection{Provenienze ed esiti dei percorsi formativi}

Come abbiamo proceduto per il percorso formativo di II grado, anche in questo caso abbiamo voluto indagare come la conoscenza della lingua possa influire sull'esito del percorso formativo accademico. Il gruppo degli studenti internazionali, arrivati quindi in Italia per frequentare l'università, ha dichiarato nel 76,5\% dei casi di aver avuto una conoscenza nulla o minima della lingua italiana al proprio ingresso nel Paese. In compenso, tale dato viene controbilanciato dalla percentuale di studenti che ha frequentato dei corsi di lingua italiana: essi sono 1'82\%, mentre per gli studenti arrivati in Italia in età scolare questa percentuale arrivava solo al $24 \%$.

12. Il numero di studenti stranieri che richiede il corso per il livello B2 è estremamente esiguo. 
Mettendo in relazione la condizione degli studenti con la media conseguita negli esami di profitto (espressa in trentesimi) possiamo osservare alcuni primi dati indicativi riguardo al rapporto fra conoscenza della lingua e gli esiti del percorso formativo: gli studenti internazionali hanno la media più bassa, con 23,5/30 (deviazione std. 4,947), media che sale a 24,0/30 (deviazione std. 5,2) ${ }^{13}$ per gli studenti stranieri scolarizzati in Italia (che, quindi, hanno avuto più tempo per poter imparare la lingua italiana) e a 25,6/30 (deviazione std. 3,2) nel gruppo degli studenti italiani (Anova con sig. 0,000$)$. Vi è anche una differenza nella votazione media fra gli studenti provenienti dalle varie aree geografiche, differenza probabilmente dovuta anche a quanto la lingua d'origine differisce rispetto a quella italiana. Gli studenti provenienti dall'Europa occidentale risultano avere il dato più alto, con una media di 24,9/30 (deviazione std. 1,9) e al $3^{\circ}$ posto troviamo, con una media di 24,5 (deviazione std. 1,5), gli studenti provenienti dall'America centrale e meridionale. Troviamo un'eccezione a tale ragionamento nel sottogruppo degli studenti provenienti dal Medio Oriente che, nonostante le rilevanti differenze fra la lingua d'origine, non neolatina, e quella italiana, si attestano al $2^{\circ}$ posto con una media di 24,5/30 (deviazione std. 1,4). In tale classifica troviamo agli ultimi posti gli studenti provenienti dall'Asia centro-orientale e sud-orientale, con una media di 23,3/30 (deviazione std. 7,9) ed infine dall'Africa subsahariana, con un dato che si attesta a 22,7/30 (deviazione std. 5,9) (Anova con Sig. ,000). Il sottogruppo che detiene la media più bassa, proveniente dall'Africa subsahariana, è anche quello oggetto, in maggior misura, di atteggiamenti pregiudiziali: 1' $80 \%$ di tali studenti riferisce di aver subito degli atti discriminatori, dato che risultava essere ancora più di impatto se comparato con quello medio degli studenti con cittadinanza non italiana, che si attesta al 53,9\%. Tali dati potrebbero portare a riflettere su un'ipotesi di correlazione fra la discriminazione degli studenti di altra cittadinanza e l'esito del loro percorso formativo che sarà presa in esame, nello specifico, nel capitolo 13.

\subsection{Le motivazioni nel percorso di studi universitari}

Le motivazioni sono un insieme di fattori che sollecitano ed orientano l'azione individuale, caratterizzate da una certa quota di eccitazione necessaria per iniziare e portare a termine un'azione (Atkinson, 1973). Secondo un modello ormai classico, le motivazioni si possono classificare in intrinseche ed estrinseche: le prime portano ad intraprendere un'attività perché è

13. Il campione degli studenti stranieri nati in Italia che ha risposto a tale quesito è troppo esiguo per essere preso in considerazione come categoria a se stante. 
di per sé motivante, mentre le seconde sono motivazioni sostenute da rinforzi esterni, quali vantaggi, riconoscimenti o evitamento di conseguenze spiacevoli. Si precisa che tale classificazione dicotomica è stata nel tempo ampliata e superata da diversi autori (Deci e Ryan, 1985; 2000).

Le motivazioni sono state rilevate attraverso un quesito in cui si chiedeva agli studenti, sia italiani sia di cittadinanza non italiana, quanto fossero d'accordo in una scala da 1 a 4 con le motivazioni elencate, di cui due di tipo intrinseco e quattro di tipo estrinseco. Comparando la scelta delle varie motivazioni osserviamo una preferenza generale per il gruppo delle motivazioni intrinseche e, fra quelle estrinseche, prevale la motivazione con una maggior "connotazione personale", che è quella di trovare il lavoro che più piace. Risulta molto indicativo il simile grado di preferenza dei fattori stimolanti nei due campioni, con una media più alta di approvazione negli studenti con cittadinanza non italiana (Graf. 3). Tali livelli di gradimento si discostano maggiormente in due punti: "poter trovare un lavoro ben pagato" e "tenermi occupato, visto che ancora non riesco a trovare un lavoro", anche se si precisa che quest'ultima motivazione è stata quella che in entrambi i campioni ha ricevuto meno apprezzamento (1,66 per il primo campione, con deviazione sdt di 0,886 e 1,47 per il secondo campione, con deviazione sdt 0,747 ). Riguardo alle motivazioni legate al "poter trovare un lavoro ben pagato" e al "dare soddisfazione ai famigliari", risulta interessante una testimonianza di uno studente proveniente dall'area dell'Europa balcanica e orientale: «Genitori insegnanti ingannati a venire in Italia con la promessa

\section{Graf. 3 - Motivazioni allo studio}

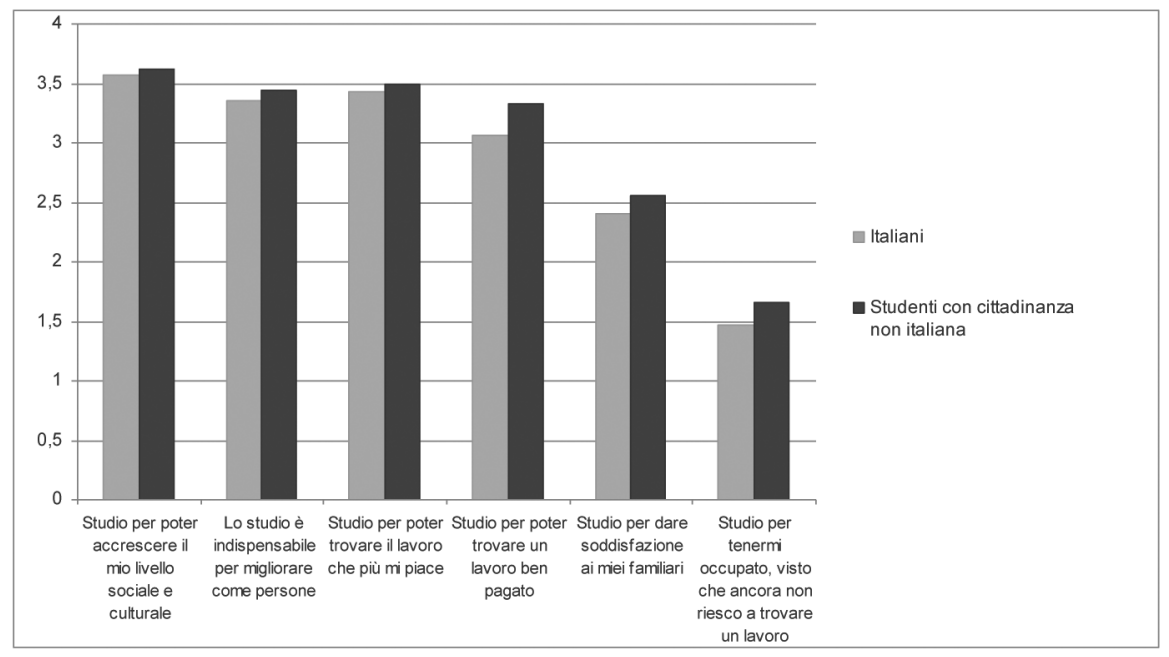


di stipendi più abbondanti... non avuto il quanto promesso in quanto le loro lauree qui non sono valide qui, sono rimasti in Italia per far studiare me e mio fratello in modo da far avere a noi lauree valide in tutta Europa $»^{14}$.

\subsection{Proposte di intervento}

«I minori stranieri, come quelli italiani, sono innanzitutto persone, e in quanto tali, titolari di diritti e doveri che prescindono dalla loro origine nazionale». Esordisce così la prima parte delle "Linee guida per l'accoglienza e l'integrazione degli alunni stranieri", pubblicate dal MIUR nel febbraio 2014, che fa seguito alle omonime linee guida pubblicate nel 2006 (MIUR, 2006; 2014). Alle linee guida ministeriali, nonostante esse abbiano svolto un importante ruolo di riferimento, in molti casi non sono state seguite nella pratica le azioni suggerite. Un esempio riguarda l'insegnamento della lingua italiana: le linee guida prevedevano l'istituzione di laboratori linguistici con percorsi e strumenti per l'insegnamento intensivo dell'italiano, eppure, nonostante il sottogruppo degli studenti stranieri scolarizzati in Italia abbia dichiarato una conoscenza quasi inesistente della lingua al suo arrivo nella scuola italiana, solo il $24 \%$ di essi ha svolto dei corsi ad hoc. Considerato che il rischio di abbandono scolastico è doppio per gli studenti stranieri rispetto a quelli con cittadinanza italiana, e che il livello di conoscenza della lingua può interagire con l'esito del percorso formativo, il primo passo per supportare il percorso scolastico e universitario è quello di potenziare i corsi di lingua ${ }^{15}$, rendendoli fortemente consigliati se non obbligatori. Di questo non gioverebbe solo il singolo alunno, ma l'intera comunità, soprattutto per quanto concerne il dramma dell'abbandono scolastico: la Comunità Europea stessa ha inserito tra i cinque obiettivi principali della Strategia Europa 2020 quello di ridurre al $10 \%$ la quota di abbandoni scolastici. Questo perché gli studenti che abbandonano il percorso formativo «sono più soggetti alla disoccupazione, hanno bisogno di più sussidi sociali e sono ad alto rischio di esclusione sociale, con conseguenze sul benessere e la salute. Inoltre, tendono a partecipare meno ai processi democratici» ${ }^{16}$.

14. Durante un'audizione al Senato della Repubblica, svoltasi nell'ottobre 2011, emersero alcune criticità dell'attuale sistema universitario, fra cui l'insufficiente riconoscimento dei titoli di studio rilasciati da Paesi terzi. Fonte: Audizione del Governo, Legislatura $16^{\mathrm{a}}-7^{\mathrm{a}}$ Commissione permanente, Resoconto sommario n. 333 del 19 ottobre 2011.

15. Come riportato anche nell'art. 5 del DPR n. 89/2009 e nella Circolare Ministeriale n. $8 / 2013$.

16. J. Ottaviani, "In Italia uno studente su tre non finisce le scuole superiori”, in L'Internazionale, 10 febbraio 2015 (www.internazionale.it/opinione/jacopo-ottaviani/2015/02/20/scuola-studenti-italia-abbandono). 
Accogliamo positivamente la presenza, nelle linee guida per l'accreditamento delle sedi di corsi di studi del $2014^{17}$, di un paragrafo legato agli studenti universitari in cui si afferma che la presenza di studenti di cittadinanza non italiana all'interno delle scuole e nei corsi universitari, sono l'indiscutibile segnale di un'immigrazione che, stabilizzandosi, punta sempre di più all'istruzione come ascensore sociale per i propri giovani. Ciò deve portare il Paese ad attuare delle strategie nei confronti di tali studenti, atte ad evitare delusioni e difficoltà che porterebbero ad una piena integrazione. Tale ragionamento si è reso necessario anche considerato l'aumento degli studenti stranieri specie di seconda generazione, rispetto agli scorsi decenni, in cui vi era una maggior percentuale di studenti internazionali.

Nel sistema universitario nazionale e locale, quali possono essere queste strategie? A livello nazionale, considerato anche il recente interesse mostrato dal MIUR e dall'ANVUR riguardo ai corsi di formazione in ingresso e in servizio rivolti ai docenti, potrebbe essere opportuno l'inserimento fra essi di moduli legati all'insegnamento e ad un corretto processo valutativo in presenza di studenti di cittadinanza non italiana e/o non italofoni, in modo da mettere a disposizione dei docenti degli strumenti adeguati per poter supportare il loro percorso formativo.

A livello locale, considerato anche che durante il primo periodo di permanenza all'università gli studenti internazionali spesso non posseggono le competenze linguistiche necessarie ad affrontare i corsi universitari e i vari iter burocratici, potrebbe essere utile la presenza di una versione in inglese dei singoli siti istituzionali degli atenei nonché della modulistica. Facendo particolare riferimento all'ateneo aquilano, un'ulteriore strategia potrebbe essere l'estensione del servizio di tutorato per gli studenti in mobilità anche agli studenti con cittadinanza non italiana che ne facciano richiesta e/o l'apertura di sportelli informativi dedicati a tale categoria all'interno dei singoli poli didattici.

17. Indicatore AQ1.D.2 delle "Linee guida per l'accreditamento delle sedi di corsi di studi" emanate dall'ANVUR in data 24.04.2014. In esse viene riportato il seguente indicatore: "L'ateneo organizza corsi di formazione e di aggiornamento destinati ai docenti sugli aspetti pedagogici e dell'insegnamento universitario, le nuove tecnologie a sostegno dell'apprendimento e la docimologia?". 


\title{
10. Studiare all'Università dell'Aquila: qualità di servizi e attrattività
}

\author{
di Arianna Fiorenza, Ilaria Salvati ${ }^{1}$
}

\subsection{La scelta della sede universitaria per gli studenti italiani e di altra cittadinanza}

Ai fini della nostra indagine abbiamo ritenuto importante capire quali siano i fattori di maggiore attrattività dell'Università dell'Aquila agli occhi degli studenti italiani e di cittadinanza non italiana. Abbiamo pertanto dedicato a questo tema una sezione del questionario, ponendo diverse domande miranti ad investigare le modalità attraverso cui gli studenti sono venuti a conoscenza dell'ateneo, le motivazioni che li hanno portati a sceglierlo, la loro percezione della qualità complessiva.

Per quanto riguarda la prima domanda ("Come e dove hai ottenuto informazioni sull'Università dell'Aquila?”) per più del 50\% degli studenti intervistati, sia italiani che non, familiari e amici hanno rappresentato la maggiore fonte di informazione. I due campioni rivelano una sostanziale somiglianza anche relativamente alle altre risposte fornite riguardo alle opzioni selezionate. In entrambi i casi, infatti, Internet è al secondo posto tra le modalità di ricerca di notizie sull'ateneo, seguito dalla scuola. L'unica, prevedibile, differenza $(7,5 \%)$ tra i due gruppi sta nel fatto che una parte degli studenti proveniente dall'estero è venuta a conoscenza della nostra università attraverso l'ambasciata o il consolato. È stato interessante chiedere agli studenti di cittadinanza non italiana se fosse stato facile per loro venire in Italia. Per poco più della metà $(55,2 \%)$ è stato "molto" o "abbastanza" facile, mentre il $23 \%$ ha dichiarato di averlo trovato "poco facile" e il 21,7\% addirittura "difficile". Alcuni, 60 su 185, hanno riportato la loro esperienza. Stando a quanto dichiarato, le principali problema-

1. I paragrafi 10.1. e 10.2. vanno attribuiti a Ilaria Salvati, mentre il paragrafo 10.3 ad Arianna Fiorenza. 
tiche riscontrate sono legate alla burocrazia eccessiva e a procedure molto lunghe. Inoltre, per alcuni, raggiungere un'ambasciata italiana è stato difficoltoso ed ha richiesto un discreto dispendio di tempo e denaro. Una volta arrivati nel nostro Paese, le complicazioni maggiormente sperimentate sono state quelle legate alle difficoltà di integrazione, al superamento della barriera linguistica e al confronto con episodi di discriminazione (cfr. cap. 13). Da un punto di vista amministrativo, gli ostacoli incontrati più frequentemente sono stati quelli relativi al rinnovo del titolo di soggiorno e al riconoscimento del titolo di studi ottenuto in patria, motivo per cui molti studenti hanno dovuto ricominciare daccapo il loro percorso accademico. Dagli studenti che, invece, si trovano in Italia da diversi anni, che vivono nel nostro Paese insieme alla loro famiglia, l'esperienza del trasferimento in Italia è stata descritta positivamente, probabilmente perché gestita interamente dai loro familiari nell'ambito delle pratiche utili al ricongiungimento.

Tab. 1 - Le testimonianze degli studenti di cittadinanza non italiana

1. Difficoltà riscontrate legate alla burocrazia eccessiva e a procedure molto lunghe

- Camerun - Dopo aver superato l'esame di lingua italiana, bisogna essere tra i primi 450 selezionati dall'ambasciata ed avere 5.750 euro in banca.

- Romania - È stato molto complicato rapportarsi con l'ambasciata e vi è tanta burocrazia inutile in certi momenti.

- Camerun - Un anno di corso di lingua intensivo e un esame molto al di sopra di quello che si vede una volta arrivati qua.

- Filippine - Abbiamo trovato molte difficoltà per le pratiche burocratiche, i tempi lunghissimi d'attesa e la mancanza di informazioni precise da seguire per fare i documenti.

- Iran - Sono dovuto andare nella capitale dieci volte per ottenere il mio visto. Non c'è nessun tipo di sistema per prendere un appuntamento.

- Ucraina - Le traduzione che si fanno presso ambasciata italiana costano troppo e l'attesa e lunghissima.

2. Difficoltà legate al raggiungimento dell'ambasciata

- Moldova - Al tempo l'Italia non aveva un'ambasciata nel mio Paese e dovevo andare nel Paese vicino, Romania.

- Madagascar - L'assente de l'ambasciata Italiana in Madagascar, insufficienza dell'informazione sul università rendono tutti molto difficile.

3. Difficoltà incontrate in Italia legate alle difficoltà di integrazione, al superamento della barriera linguistica e al confronto con episodi di discriminazione

- Romania - Sono in Italia da 7 anni. ... II problema "razzismo" racchiude un po" tutti gli altri.

- Grecia - Ad alcuni prof. non piace il fatto che abbiamo un accento non perfetto o sbagliare a volte mentre parliamo. 
Tra i motivi principali che hanno spinto gli intervistati ad iscriversi presso l'Università dell'Aquila, per entrambi i gruppi di studenti, vi sono l'esenzione dalle tasse e la vicinanza al luogo di residenza o alla famiglia. Quest'ultimo è il primo motivo indicato dagli italiani (35,9\%), seguito dall'esenzione dalle tasse (22,9\% a fronte del 24,8\% degli studenti di cittadinanza non italiana). Per gli studenti di cittadinanza non italiana l'importanza di restare vicini al proprio luogo di residenza e ai propri familiari è sentita dal $23,6 \%$ e un altro $21,1 \%$ ha scelto di frequentare l'Università dell'Aquila per seguire amici o familiari. Questi dati ci spingono ad una riflessione di tipo sociologico di un certo rilievo: sia i figli di migranti sia coloro che vengono da lontano sono tendenzialmente portati a stabilirsi in un luogo in cui hanno delle conoscenze, dove possono avere un punto di riferimento dato da persone care, secondo il modello classico della catena migratoria, che, dunque, almeno in parte, spiega i fattori di attrazione che regolano i flussi migratori per motivi di studio.

\subsection{La percezione della qualità della formazione}

La percezione della qualità del sistema universitario complessivamente inteso è stata misurata attraverso 8 indicatori (scale da 1 a 5) riferiti a tutorato, servizi di segreteria, diffusione delle informazioni, didattica, strutture di supporto allo studio, diritto allo studio, logistica e modulistica. Riportiamo nella Tabella 2 le medie dei diversi indicatori rilevati, sui due gruppi rapportate, per rendere maggiormente leggibili i dati, su una scala $0-10$. L'analisi della varianza ci ha permesso di individuare per quasi tutti gli aspetti indagati (tranne che per la didattica e per il sistema di diffusione delle informazioni) differenze significative nella valutazione tra il campione degli italiani e il campione degli studenti di cittadinanza non italiana (Tab. 2); questi ultimi sembrano valutare meglio dei primi i servizi offerti dall'università².

2. All'interno del campione di studenti di cittadinanza non italiana non sono state riscontrate differenze significative tra studenti internazionali e studenti stranieri scolarizzati in Italia. 
Tab. 2 - La qualità dell'ateneo per gli studenti italiani e di altra cittadinanza (punteggi su scala 0-10)

\begin{tabular}{lccc}
\hline & $\begin{array}{c}\text { Studenti } \\
\text { italiani }\end{array}$ & $\begin{array}{c}\text { Studenti di } \\
\text { cittadinanza } \\
\text { non italiana }\end{array}$ & $\begin{array}{c}\text { Anova } \\
\text { con F } \\
\text { significativo }\end{array}$ \\
\hline Tutorato & 3,5 & 2,9 & Sì̀ \\
\hline Servizi di segreteria & 4,5 & 3,7 & Sì̀ \\
\hline Diffusione delle informazioni & 4,7 & 4,2 & Sì \\
\hline Didattica & 5,7 & 5,7 & No \\
\hline Strutture di supporto allo studio & 4,7 & 3,8 & Sì \\
\hline Diritto allo studio & 3,7 & 2,9 & Sì \\
\hline Logistica & 4 & 3,4 & Sì \\
\hline Modulistica & 4,2 & 3,6 & Sì \\
\hline
\end{tabular}

Sulla percezione della qualità l'indicatore con punteggio medio più alto, che più soddisfa entrambi i gruppi, è quello relativo alla didattica; una tendenza analoga è stata già riscontrata in una precedente indagine del 2011 sulla qualità della vita e sulla motivazione allo studio degli studenti dell'Università dell'Aquila dopo il sisma 2009 (Isidori e Vaccarelli, 2012). La percezione che hanno gli studenti sembra evidenziare l'importanza che l'università attribuisce alla qualità della sua didattica. La valutazione media è di $2,7(5,7$ su scala $0-10)$ e all'ultimo posto in termini di qualità per entrambi i gruppi di studenti troviamo i servizi di tutorato. Infine, per gli studenti italiani risulta praticamente irrilevante la presenza di accordi o progetti internazionali, che invece ha attratto all'Aquila il 5\% degli studenti di cittadinanza non italiana. Per quanto riguarda le problematiche riscontrabili nello studio universitario, sono stati individuati 6 indicatori riguardanti le difficoltà nella comprensione relative alle modalità degli esami, sia scritti sia orali, alle lezioni dei docenti e ai libri di testo (Tab. 3). La maggioranza degli appartenenti ai due gruppi ha dichiarato di avere "poca" o "nessuna" difficoltà nel comprendere i libri e i materiali didattici (71,9\% studenti di cittadinanza non italiana, 78,6\% italiani) e nel sostenere i test scritti $(74,2 \%$ - 80,1\%). Significativa (Chi Quadro Sig. ,000) è la differenza riguardante la difficoltà nel sostenere gli esami orali e nel rispondere alle domande aperte durante le prove scritte. 
Tab. 3 - Difficoltà incontrate nell'affrontare lo studio universitario dagli studenti

\begin{tabular}{lcccc}
\hline & $\begin{array}{c}\text { Studenti } \\
\text { internazionali }\end{array}$ & $\begin{array}{c}\text { Studenti } \\
\text { stranieri }\end{array}$ & $\begin{array}{c}\text { Studenti } \\
\text { italiani }\end{array}$ & $\begin{array}{c}\text { Anova } \\
\text { con F } \\
\text { significativo }\end{array}$ \\
\hline & Media & Media & Media & Sì \\
\hline $\begin{array}{l}\text { Comprendere i professori } \\
\text { quando spiegano a lezione }\end{array}$ & 4,3 & 2,9 & 3,4 & No \\
\hline $\begin{array}{l}\text { Comprendere i libri e i } \\
\text { materiali didattici }\end{array}$ & 3,8 & 2,0 & 2,0 & Sì \\
\hline $\begin{array}{l}\text { Rispondere alle domande } \\
\text { aperte durante gli esami }\end{array}$ & 4,8 & 2,0 & 1,9 & Sì \\
\hline $\begin{array}{l}\text { Rispondere ai test scritti } \\
\text { durante gli esami }\end{array}$ & 4,0 & 1,9 & 2,0 & Sì \\
\hline $\begin{array}{l}\text { Comprendere le domande } \\
\text { poste durante gli esami } \\
\text { orali }\end{array}$ & 4,0 & 1,9 & 1,9 & Sì \\
\hline $\begin{array}{l}\text { Rispondere alle domande } \\
\text { negli esami orali }\end{array}$ & 5,0 & 2,0 & 1,9 & Sì \\
\hline \begin{tabular}{l} 
Indice difficoltà studio \\
\hline
\end{tabular} & 5,5 & 4,6 & 4,6 & \\
\hline
\end{tabular}

Si è cercato di approfondire l'analisi dei dati attraverso la distinzione tra studenti internazionali e studenti stranieri scolarizzati in Italia, elemento che ha effettivamente condizionato e differenziato significativamente le medie riferite ad ognuno degli indicatori. Nella Tabella 4 sono riportati i punteggi su scala $0-10$, e viene indicato se l'analisi della varianza ha ottenuto valori statisticamente significativi. È possibile notare un diverso comportamento delle variabili esaminate tra gli studenti internazionali e gli studenti stranieri scolarizzati in Italia. Nello specifico, gli studenti internazionali sono quelli che dichiarano mediamente maggiori difficoltà sui singoli indicatori, nonché sull'indice complessivo, mentre gli studenti stranieri scolarizzati in Italia tendono ad uniformarsi al gruppo degli studenti italiani. Ciò sta a significare che le competenze in lingua italiana rappresentano uno dei principali punti di debolezza degli studenti internazionali nel momento in cui si trovano ad affrontare lo studio universitario; le difficoltà maggiori sono state riscontrate relativamente nella produzione orale e scritta (indicate dalle opzioni "Rispondere alle domande negli esami orali" e "Rispondere alle domande aperte durante le prove di esame"), più che nelle competenze di comprensione. Questo fenomeno viene ulteriormente confermato da una correlazione di $-0,23$ (Spearman, con significatività ,003) tra il livello linguistico e l'indice di difficoltà (al crescere del primo tende evidentemente a ridursi la difficoltà nello studio). 
Per quanto riguarda la frequenza delle lezioni e dell'ambiente universitario, la maggioranza degli intervistati ha dichiarato di frequentare "molto" (41,6\% degli studenti di cittadinanza non italiana a fronte del 36,9\% di studenti italiani) o "abbastanza" (34,2\% di cittadinanza non italiana; 30,2\% di cittadinanza italiana), mentre le percentuali relative a chi frequenta "poco" $(15,2 \% ; 18,3 \%)$ o "non frequenta" $(8,7 \% ; 14,6 \%)$ sono ben inferiori. Gli studenti di altra cittadinanza frequentano maggiormente l'ateneo rispetto agli studenti italiani. Se uniamo le voci "molto" e "abbastanza" otteniamo una differenza di 8,7 punti percentuali. Sarebbe stato lecito immaginare che gli studenti di cittadinanza non italiana frequentino con più costanza a causa di maggiori difficoltà nello studio, analizzate in precedenza, ma dal calcolo del Chi Quadro non si evince una significatività tale da evidenziare questa tendenza (per motivi di sintesi, non si riportano dati e tabelle). Si potrebbe ipotizzare che essi utilizzano le lezioni e gli spazi dell'università anche come riferimento importante per la socialità.

\subsection{La valutazione delle attività a favore degli studenti di cittadinanza non italiana}

Nel giudicare l'operato dei rappresentanti degli studenti, gli italiani e gli stranieri si sono dimostrati tendenzialmente d'accordo. Più di un terzo degli appartenenti ad entrambi i campioni (37,1\% di cittadinanza non italiana e $40,6 \%$ italiani), infatti, ha valutato i rappresentanti con un voto di media (3 su una scala da 1 a 5).

Alla domanda relativa alla valutazione delle azioni delle organizzazioni studentesche a favore degli studenti di cittadinanza non italiana, le percentuali riferite alle varie risposte variano molto tra i due campioni ed emerge una differenza significativa (Chi Quadro Sig. ,000) tra la loro percezione (Tab. 4). Bisogna comunque tener conto del fatto che, alla categoria oggetto del quesito, non appartengono solo i rappresentanti degli studenti, ma anche le organizzazioni studentesche di orientamento culturale. Per la maggior parte degli studenti italiani $(58,6 \%)$ le organizzazioni studentesche tengono abbastanza in considerazione i problemi legati agli studenti di altra provenienza. Più della metà di questi ultimi (74,5\%), invece, ritiene che le organizzazioni si preoccupino poco dei loro problemi. Trattandosi di problematiche proprie di uno dei due gruppi, questa differenza di percezione è comprensibile. Gli studenti di cittadinanza non italiana, essendo maggiormente a conoscenza delle difficoltà da essi stessi riscontrate e di come queste vengano o meno risolte, sono portati a ritenere che quanto fatto per loro non sia abbastanza. 
Tab. 4 - Percezione dell'operato delle organizzazioni studentesche

\begin{tabular}{ccccc}
\hline \multicolumn{2}{c}{$\begin{array}{c}\text { Studenti di cittadinanza } \\
\text { non italiana }\end{array}$} & \multicolumn{2}{c}{ Studenti italiani } & Chi Quadro \\
\hline Poco/ & Molto/ & Poco/ & Molto/ & Sig. \\
Per niente & Abbastanza & Per niente & Abbastanza &, 000 \\
$74,5 \%$ & $25,4 \%$ & $41,5 \%$ & $58,6 \%$ &, \\
\hline
\end{tabular}

Anche nel giudizio riguardante le azioni dell'Università dell'Aquila a favore degli studenti di altra provenienza, questi ultimi tendono ad essere maggiormente critici rispetto a quanto facciano i colleghi italiani (Tab. 5). La metà dei primi, infatti, ha dato una valutazione negativa (il $20,5 \%$ ha valutato con 1 e il 31,1\% con 2). Tra gli italiani, invece, quasi la metà (49\%) ha scelto di giudicare l'operato del nostro ateneo a favore degli studenti di altra provenienza con 3 (scala 1-5), e le percentuali relative a valutazioni più positive sono maggiori rispetto a quanto espresso dagli studenti non italiani (17,8\% contro $11,7 \%$ per il voto 4 e $3 \%$ contro $1,9 \%$ per il voto 5 ).

Tab. 5 - Percezione delle azioni dell'Università dell'Aquila a favore degli studenti di cittadinanza non italiana, da parte di entrambi i gruppi

\begin{tabular}{cccccc}
\hline \multicolumn{2}{c}{$\begin{array}{c}\text { Studenti di cittadinanza } \\
\text { non italiana }\end{array}$} & \multicolumn{2}{c}{ Studenti italiani } & \multicolumn{2}{c}{ Anova } \\
\hline Media & Dev. Std. & Media & Dev. Std. & F & Sig. \\
2,42 &, 989 & 2,83 &, 949 & 21,059 &, 000 \\
\hline
\end{tabular}

Agli italiani è stato anche chiesto di spiegare le motivazioni delle loro valutazioni sulle azioni a favore degli studenti stranieri da parte dell'ateneo, e il dato più rilevante sta nel fatto che ben il 32,5\% dei rispondenti dichiara di "non sapere", di non essere a conoscenza di azioni adottate dalla nostra università a favore degli studenti di cittadinanza non italiana. La mancanza di elementi su cui operare una valutazione è il motivo che ha spinto molti degli intervistati a dare un giudizio medio ( 3 , su scala da 1 a 5 ). Che ci sia confusione riguardo a cosa effettivamente l'ateneo aquilano faccia per gli studenti di cittadinanza non italiana (ad eccezione dei beneficiari di una borsa di studio Erasmus) lo rivelano anche le risposte dei restanti due terzi del campione, che in alcuni casi risultano diametralmente opposte.

Nel Grafico 1, osserviamo che il $2 \%$ degli studenti ha dichiarato che il nostro ateneo agisce "nella norma", per quanto gli compete, in supporto 
degli studenti stranieri. A sinistra di tale valore si trovano le valutazioni negative, a destra invece quelle positive. Il $16 \%$ degli studenti italiani pensa che non ci siano abbastanza iniziative a favore dell'integrazione da parte dell'università, il 12\% ritiene invece che siano sufficienti. Il $13 \%$ ritiene che i servizi e i corsi in inglese siano scarsi o assenti, il 10\% invece sostiene che i docenti sono disponibili a tenere lezione anche in lingua inglese. Il $7 \%$, inoltre, afferma che il personale docente e amministrativo sia indifferente alle problematiche relative agli studenti stranieri, mentre un $9 \%$ ritiene che in realtà in ateneo ci sia un buon clima favorevole all'integrazione. Preponderante è la quota di chi sostiene che i servizi e i corsi siano inadeguati per gli studenti stranieri (14\% contro $2 \%$ ), mentre il 3\% lamenta la scarsa pubblicità che viene data alle iniziative promotrici dell'integrazione. Da segnalare, infine, un $4 \%$ di studenti che ritiene che l'Università dell'Aquila non soltanto faccia molto per gli studenti di cittadinanza non italiana, ma addirittura che ci siano dei favoritismi nei loro confronti quali esami più facili, agevolazioni fiscali e maggiori servizi per garantire loro il diritto allo studio. Emerge un quadro, dunque, confusionario e incoerente, che segnala l'esigenza di una progettazione mirata delle politiche accademiche in favore degli studenti di cittadinanza non italiana.

Graf. 1 - Valutazioni e considerazioni degli studenti sulle iniziative a favore delle iniziative per gli studenti di cittadinanza non italiana (domanda aperta)

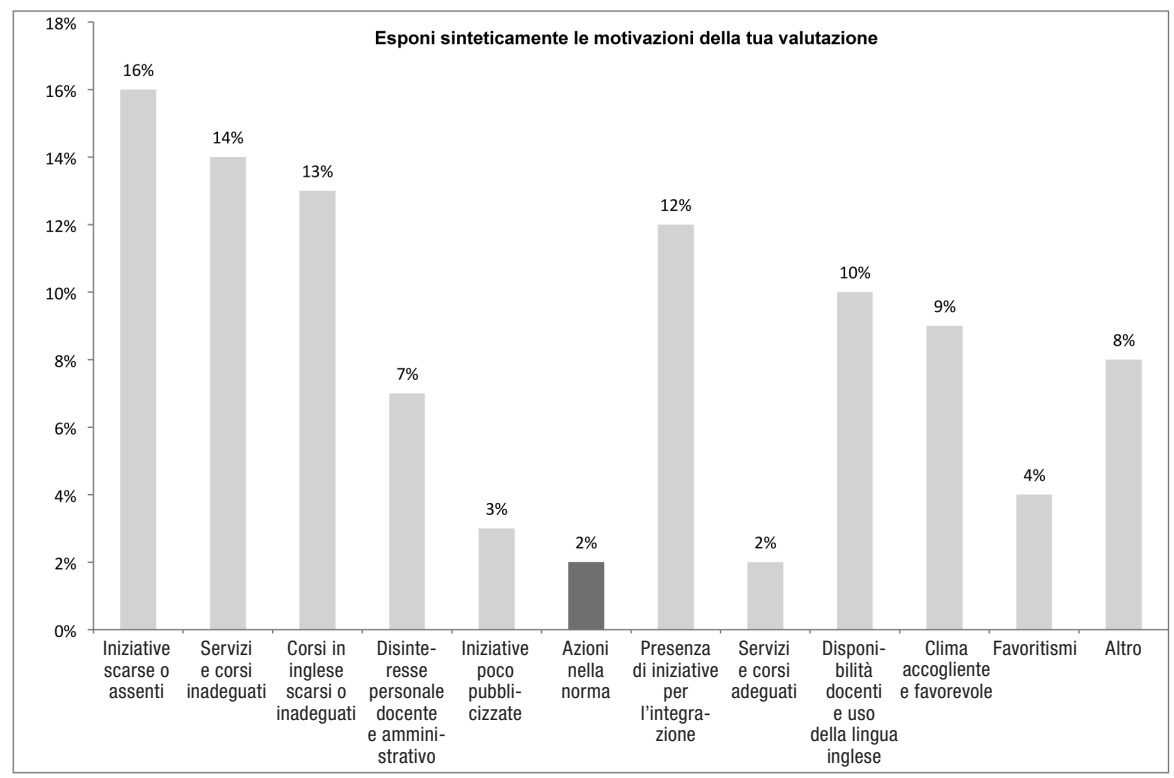


Nella parte finale del questionario relativa agli studi universitari, è stato chiesto agli studenti di entrambi i gruppi di presentare le loro eventuali proposte per migliorare le condizioni di vita e di studio degli studenti stranieri presso l'Università dell'Aquila. Hanno fornito la loro opinione il 19\% degli italiani intervistati e il 25,7\% degli studenti di altra cittadinanza, differenza che segnala evidentemente una maggiore attenzione da parte dei diretti interessati.

Per gli studenti italiani, le iniziative volte a migliorare le condizioni di vita e di studio dei colleghi dovrebbero essere soprattutto indirizzate all'organizzazione di momenti di incontro e condivisione e all'abbattimento delle barriere linguistiche, sia rafforzando l'insegnamento della lingua italiana sia offrendo corsi in lingua inglese che, secondo molti, andrebbero a beneficio anche degli stessi italiani (Tab. 6). Si tratta di suggerimenti condivisi anche da una buona parte degli studenti di cittadinanza non italiana, i quali, però darebbero la priorità ad una revisione dell'organizzazione dell'ateneo (segnalata anche da quasi il $15 \%$ degli italiani) e alla messa a disposizione di servizi dedicati quali quelli di tutorato. Percentuali inferiori di studenti consigliano di incrementare i servizi allo studio, di garantire una maggiore professionalità da parte del personale e di migliorare i trasporti. Un 5\% circa di studenti di cittadinanza non italiana ritiene, inoltre, che bisognerebbe prevedere maggiori agevolazioni per i non italiani in termini fiscali e di benefici quali gratuità dell'alloggio o della mensa. Non man-

\section{Tab. 6 - Le azioni proposte dagli studenti}

\section{Organizzazione di momenti di incontro e condivisione e per l'abbattimento delle barriere linguistiche}

- Attraverso attività mirate alla socializzazione, bisognerebbe creare una maggiore coesione.

- Organizzare degli incontri culturali tra studenti italiani e stranieri.

- Si potrebbero organizzare incontri di scambi culinari e gastronomici.

- Organizzazione di gruppi per poter imparare lingue diverse.

- Si potrebbe pubblicizzare il ruolo dell'esperanto.

- "Olimpiadi" universitarie mischiando stranieri e studenti italiani, lo sport unisce.

2. Rafforzando l'insegnamento della lingua italiana offrendo corsi in lingua inglese

- Ci dovrebbero essere più corsi offerti in inglese, oppure i professori dovrebbero essere in grado di fornire del materiale in inglese.

- La biblioteca dovrebbe avere più copie di libri in inglese.

- Nessuna delle persone che lavorano presso la segreteria studenti è in grado di parlare inglese.

- Sarebbe molto utile inserire un corso di lingua italiana. Siccome il centro linguistico non lo svolge io ho dovuto frequentare uno appagamento.

\section{Organizzazione dell'ateneo}

- Struttura universitaria da rivedere completamente.

- Garantire almeno gli avvisi anche in lingua inglese. 
ca, infine, una piccola percentuale di italiani che ritiene che l'università dovrebbe preoccuparsi principalmente degli studenti loro connazionali, in un atteggiamento purtroppo abbastanza ricorrente quando si parla di immigrati.

Dalla nostra indagine risulta che sono diverse le difficoltà che incontrano gli studenti di altra cittadinanza nel venire a studiare in Italia. Alcune sono legate al loro Paese di appartenenza e sono soprattutto di tipo burocratico, altre emergono nel contesto di accoglienza. Per quanto riguarda queste ultime, le principali problematiche sono relative alla lingua, alla burocrazia, al reperimento di informazioni dai vari uffici dell'ateneo e alla didattica, soprattutto nel sostenimento degli esami. Sicuramente una maggiore sensibilità da parte del personale tecnico-amministrativo e un diffuso utilizzo della lingua inglese faciliterebbero molto l'inserimento degli studenti stranieri nell'ambiente universitario. A tal fine un ruolo importante è, naturalmente, svolto dai docenti, i quali potrebbero venire incontro alle difficoltà degli studenti di altra cittadinanza nelle prove orali e nelle prove scritte a risposta aperta, prevedendo delle prove differenti o delle modalità d'esame diverse almeno per il primo anno, come ad esempio i test a risposta multipla che neutralizzano gli effetti sulla valutazione delle competenze produttive in lingua italiana (cfr. Vaccarelli, 2003). Nonostante le difficoltà appena accennate, gli studenti di cittadinanza non italiana scelgono comunque di studiare all'Università dell'Aquila, soprattutto per la qualità della sua didattica. Sono più critici, invece, nei confronti dei servizi offerti dall'ateneo, tra i quali quello del tutorato. Da diverse risposte, inoltre, si è notato come gli studenti stranieri sentano la necessità di stare vicini a persone care e ciò ha influito sulla scelta dell'università e influisce, soprattutto, sul desiderio di frequentare maggiormente i locali dell'ateneo. Questo bisogno e questo desiderio di socializzare sono sentiti sia dagli studenti di cittadinanza non italiana sia da quelli italiani. Tra le proposte per il miglioramento delle condizioni di vita degli studenti avanzate da entrambi i gruppi, infatti, ce ne sono diverse legate a questo aspetto. Organizzare eventi e momenti ricreativi, di incontro e di condivisione, potrebbe essere un buon suggerimento per i rappresentanti dell'università e delle organizzazioni studentesche, le quali sono state ritenute poco attente nei confronti delle problematiche degli stranieri. 


\title{
11. Alcuni aspetti della socialità in ambito universitario
}

\author{
di Arianna Fiorenza, Chiara Ciccozzi ${ }^{1}$
}

\subsection{Introduzione}

La socialità, intesa come l'insieme dei rapporti che si creano fra gli individui che fanno parte di un ambiente, nasce e si sviluppa in noi fin dall'infanzia. Ognuno di noi, infatti, è costantemente e quotidianamente impegnato nei rapporti interpersonali e negli scambi comunicativi. Quando comunichiamo, condividiamo opinioni, emozioni, stati d'animo ed esperienze. Elementi, questi, che portano alla creazione di legami più o meno autentici.

Per Maslow (2010), in ogni essere umano esiste il bisogno di essere amato e di fare parte di un gruppo; tale bisogno rientra nel momento intermedio della sua nota gerarchia dei bisogni e delle necessità. Questa scala di bisogni è suddivisa in cinque differenti livelli, dai più elementari ai più complessi. Tra i più complessi abbiamo quelli di carattere sociale e in particolare il bisogno di appartenenza che rappresenta l'aspirazione di ognuno di noi a essere un membro della comunità. Se nella ricerca pedagogica e psicosociale si è data una certa rilevanza agli studi sulla socialità in ambito scolastico ${ }^{2}$, ci sembra che il tema della socialità in ambito universitario, soprattutto riferita ai contesti interculturali, sia ancora poco studiato. Un esempio di indagine sugli studenti universitari di origine non italiana, che ha per oggetto anche le relazioni sociali, è stato condotto nell'ambito dell'Università di Genova (Lagomarsino, Ravecca, 2014). La ricerca nasce dall'intento di capire quali sono i percorsi degli studenti di cittadinan-

1. I paragrafi 11.1. e 11.3. vanno attribuiti ad Arianna Fiorenza, mentre i paragrafi 11.2. e 11.4. a Chiara Ciccozzi.

2. Ciò anche in relazione ad una maggiore circoscrizione dei gruppi studiati, che coincidono il più delle volte con la classe scolastica, ben definita e costante rispetto alle caratteristiche delle "classi" accademiche. Nella scuola, infatti, tecniche come quella sociometrica possono essere agevolmente applicate anche in relazione alle relazioni interculturali (cfr. Nigris, 2002). 
za non italiana, i motivi dei successi e degli insuccessi e gli elementi da considerare come buoni predittori della piena integrazione sociale. Infatti è emerso che il successo formativo e l'integrazione dipendano molto da fattori legati alla posizione sociale. Gli studiosi hanno individuato, come si è già detto nel capitolo 4 , diversi profili di studenti nei quali rientrano ragazzi e ragazze con un percorso relativamente omogeneo, rispetto alle caratteristiche socio-economiche, alla storia migratoria e alle prospettive per il futuro. Tali caratteristiche sembrano incidere anche sul tipo di relazioni sociali che gli studenti di cittadinanza non italiana instaurano. Infatti è emerso che gli studenti con maggiori possibilità economiche e che non si sentono immigrati ma anzi si percepiscono come "cittadini del mondo", sono più propensi a creare legami non per appartenenza etnica, culturale o nazionale bensì per simpatia, per condivisione di interessi e di percorsi scolastici. Coloro, invece, che appartengono ad una classe sociale medio/bassa e che si sentono spersi e disorientati rispetto alla loro presenza in Italia e al loro percorso formativo, tendono a sviluppare rapporti con i membri della propria comunità. Inizialmente ciò è dovuto al desiderio di rifugio nel gruppo di appartenenza con cui condividono la lingua e spesso la religione; successivamente questa tendenza a restare chiusi nella propria comunità, li porta a uno svantaggio nell'integrazione. Nei prossimi paragrafi si riporteranno dati sulle relazioni degli studenti di cittadinanza non italiana e italiana condotto presso l'Università dell'Aquila, nell'ambito del progetto "Unidiversità", che, tra le altre cose, trovano interessanti elementi di continuità con i risultati dell'indagine condotta nel contesto genovese.

\subsection{Le relazioni all'interno dell'ambiente universitario}

L'analisi delle relazioni all'interno dell'ambiente accademico costituisce un momento fondamentale per la ricerca condotta presso l'Università dell'Aquila, poiché tra le sue finalità si ritrova la progettazione di azioni legate all'idea di convivenza interculturale e di inclusione.

I dati che riportiamo descrivono la qualità delle relazioni sociali attraverso alcune domande volte a definire le diverse situazioni in rapporto al numero di amici, al numero di conoscenti e alla nazionalità.

Agli studenti è stato chiesto: "Quante sono le persone che a livello universitario puoi considerare veri e propri amici?". Le risposte indicate sono organizzate per range (Tab. 1). È emerso che la percentuale più alta degli studenti italiani $(38,2 \%)$ ha tra i 3 e i 5 amici nell'ambiente universitario, invece, quella più alta degli studenti di cittadinanza non italiana $(36,4 \%)$ ha tra 1 e 2 amici. La differenza, come rilevata dal Chi Quadro, è significativa (Sig. ,000) e se ne può dedurre che tendenzialmente gli studenti di 
cittadinanza non italiana hanno minori opportunità di costruire amicizie nell'ambiente universitario.

Tab. 1 - Numero di amici degli studenti di cittadinanza italiana e non

\begin{tabular}{lccc}
\hline & Italiani & $\begin{array}{c}\text { Cittadinanza } \\
\text { non italiana }\end{array}$ & Differenze \\
\hline Nessuno & $12,6 \%$ & $11,7 \%$ & $0,9 \%$ \\
\hline $1-2$ & $32,4 \%$ & $36,4 \%$ & $-4,0 \%$ \\
\hline $3-5$ & $38,2 \%$ & $34,4 \%$ & $3,8 \%$ \\
\hline Più di 5 & $16,8 \%$ & $17,5 \%$ & $-0,7 \%$ \\
\hline Totale & $100 \%$ & $100 \%$ & $0 \%$ \\
\hline Chi Quadro & Valore & df & Sig. asint. (2 vie) \\
\hline di Pearson & 69,837 & 4 &, 000 \\
\hline
\end{tabular}

All'interno del campione degli studenti di cittadinanza non italiana, si nota una differenza significativa tra i due sottogruppi di studenti internazionali e studenti stranieri scolarizzati in Italia. Come osserviamo nella Tabella 2, li studenti stranieri scolarizzati in Italia tendono ad essere meno espansivi ${ }^{3}$ di

Tab. 2 - Numero di amici per studenti internazionali e studenti stranieri scolarizzati in Italia

\begin{tabular}{lccc}
\hline & \multicolumn{2}{c}{ Cittadinanza non italiana } & \multirow{2}{*}{ Differenza } \\
\cline { 2 - 3 } & Internazionali & Stranieri & \\
\hline Nessuno & $11,6 \%$ & $12,3 \%$ & $-0,7 \%$ \\
\hline $1-2$ & $33,7 \%$ & $38,5 \%$ & $-4,9 \%$ \\
\hline $3-5$ & $30,5 \%$ & $42,1 \%$ & $-11,6 \%$ \\
\hline Più di 5 & $24,2 \%$ & $7,0 \%$ & $17,2 \%$ \\
\hline Totale & $100 \%$ & $100 \%$ & $100 \%$ \\
\hline & Valore & df & Sig. asint. (2 vie) \\
\hline $\begin{array}{l}\text { Chi Quadro } \\
\text { di Pearson }\end{array}$ & 7,555 & 3 &, 056 \\
\hline
\end{tabular}

3. Utilizziamo il termine "espansione" in riferimento alla tecnica sociometrica, nella quale è inteso come il numero di persone verso le quali si è diretta almeno una scelta e dunque come indicatore della tendenza dell'individuo a "cercare" attivamente relazioni sociali (cfr. Reffieuna, 2003). 
quelli internazionali (rispondono di avere più di 5 amici nel $7 \%$ dei casi a fronte del 24,2\% degli studenti internazionali), anche in questo caso con un Chi Quadro significativamente accettabile.

I due sottogruppi presentano un andamento differente anche rispetto alla nazionalità degli amici che scelgono. Gli studenti stranieri scolarizzati in Italia mostrano un maggiore interesse a creare dei rapporti al di fuori della propria comunità di appartenenza. Esempi significativi li abbiamo nel caso dei soggetti provenienti dall'Europa balcanica e orientale, in cui troviamo una percentuale pari a $74,5 \%$ di coloro che privilegiano amicizie di nazionalità diversa dalla loro; in particolare, gli studenti rumeni, albanesi e macedoni scelgono amicizie al di fuori della loro comunità rispettivamente per l' $84,6 \%, l^{\prime} 81,3 \%$ e il $60 \%$.

Nel caso degli studenti internazionali, invece, la tendenza generale che si riscontra è quella di creare rapporti di amicizia all'interno della propria comunità, come nel caso di studenti provenienti dalla Grecia e dal Medio Oriente. Infatti, tra gli studenti di cittadinanza greca, libanese e israelo-palestinese si riscontrano valori molto elevati che riguardano quasi la totalità dei soggetti. All'interno di tale categoria, le uniche eccezioni riguardano gli studenti provenienti dall'Europa balcanica e orientale e il più esiguo gruppo proveniente l'America centrale e meridionale.

Per quanto riguarda il gruppo degli studenti di cittadinanza albanese riscontriamo una diversa tendenza nel modo di relazionarsi con gli altri. Tra loro, gli studenti internazionali, in una percentuale pari al 66,7\%, preferiscono creare legami con i membri della propria comunità; gli studenti scolarizzati in Italia, al contrario, instaurano relazioni con studenti di altra nazionalità con una percentuale pari all' $81,3 \%$. Ciò ci permette di comprendere che, in linea generale, la socialità, più che essere influenzata dalla provenienza o dalla cultura di origine, risente spesso delle diverse condizioni dei due sottogruppi di studenti: in un caso, per gli studenti scolarizzati in Italia, esiste una rete sociale preesistente e una maggiore apertura legata alla conoscenza, e forse ad un più forte radicamento, ai modelli culturali e sociali del contesto di accoglienza, nell'altro, per gli studenti internazionali, l'arrivo in Italia può essere segnato e ammortizzato dalla ricerca di connazionali e di legami comunitari, anche considerati come punti di riferimento stabile all'interno di un'esperienza che può contemplare anche momenti di shock culturale e linguistico (Schumann, 1978).

Con il test del Chi Quadro, è stato chiarito che il livello di conoscenza della lingua italiana non ha alcuna influenza sul numero di amici che si hanno a livello universitario. Al contrario, contribuisce a determinare le scelte amicali sulla base della nazionalità: gli studenti di cittadinanza non italiana che hanno una buona o ottima conoscenza della lingua italiana hanno principalmente studenti italiani come amici (Chi Quadro con Sig. ,000). 
Analizzando l'associazione tra il sesso e l'indicatore di espansione (il numero di scelte espresse dal singolo) all'interno dei due campioni, è emersa una differenza di genere, che vede gli uomini maggiormente propensi a creare legami. Per quanto riguarda le donne, il $43,3 \%$ ha dichiarato di avere tra 1 e 2 veri amici, seguito dal $33,7 \%$, che ne ha dichiarati tra 3 e 5; per quanto riguarda gli uomini, il $37,7 \%$ di loro ha dichiarato di avere tra i 3 e i 5 amici, seguito dal 30,3\% che ha dichiarato di averne più di 5 (a fronte del 6,2\% di donne). Nel caso delle donne non si osservano differenze significative di distribuzione tra le studentesse italiane, quelle scolarizzate in Italia e quelle internazionali. Nel gruppo degli uomini le sottocategorie degli studenti italiani e stranieri scolarizzati in Italia presentano una scelta che va da 3 a 5 (con percentuali pari a 39,3\% e 46,7\%), mentre per gli studenti internazionali la scelta va oltre il $5(35,3 \%)$. Si nota quindi come gli uomini, a prescindere dalla loro condizione, creino rapporti numericamente superiori rispetto a quelli delle donne, di qualunque nazionalità essi siano, mostrando così un'apertura maggiore. La stessa procedura è stata utilizzata anche con riferimento ai "conoscenti" (Tab. 3), con un range di risposte più ampie (nessuno, da 1 a 2 , da 3 a 5 , da 6 a 10, più di 10). È emerso che nel gruppo di italiani il $39,7 \%$ ha più di 10 conoscenti, il $36,9 \%$ degli studenti scolarizzati in Italia ha tra 6 e 10 conoscenti, mentre il 28,6\% degli studenti internazionali ha più di 10 conoscenti. All'interno del campione degli studenti di cittadinanza non italiana è emerso che i conoscenti connazionali sono il $18,5 \%$ per gli studenti scolarizzati in Italia, mentre per quelli internazionali il 54,6\%.

Tab. 3 - Numero di conoscenti degli studenti di cittadinanza italiana e non

\begin{tabular}{lccc}
\hline & Italiani & $\begin{array}{c}\text { Cittadinanza } \\
\text { non italiana }\end{array}$ & Differenze \\
\hline Nessuno & $3,8 \%$ & $4,5 \%$ & $-0,7 \%$ \\
\hline $1-2$ & $10,6 \%$ & $15,8 \%$ & $-5,2 \%$ \\
\hline $3-5$ & $23,7 \%$ & $21,1 \%$ & $2,6 \%$ \\
\hline $6-10$ & $22,4 \%$ & $27,0 \%$ & $-4,6 \%$ \\
\hline Più di 10 & $39,5 \%$ & $31,6 \%$ & $7,9 \%$ \\
\hline Totale & $100 \%$ & $100 \%$ & $0 \%$ \\
\hline & Valore & df & Sig. asint. (2 vie) \\
\hline $\begin{array}{l}\text { Chi Quadro } \\
\text { di Pearson }\end{array}$ & 5,832 & 4 &, 212 \\
\hline
\end{tabular}


Quanto accade per le amicizie, anche per le conoscenze gli studenti internazionali dimostrano una maggiore difficoltà ad instaurare relazioni interpersonali con studenti non connazionali. Notiamo per entrambi i campioni, studenti italiani e di cittadinanza non italiana, una maggiore apertura nell'avere un numero di conoscenti che va oltre i 5 a differenza del numero di amici che si assesta tra 3 e 5, come è facilmente riscontrabile nei Grafici 1 e 2.

Graf. 1 - Numero di amici: studenti italiani e studenti di cittadinanza non italiana

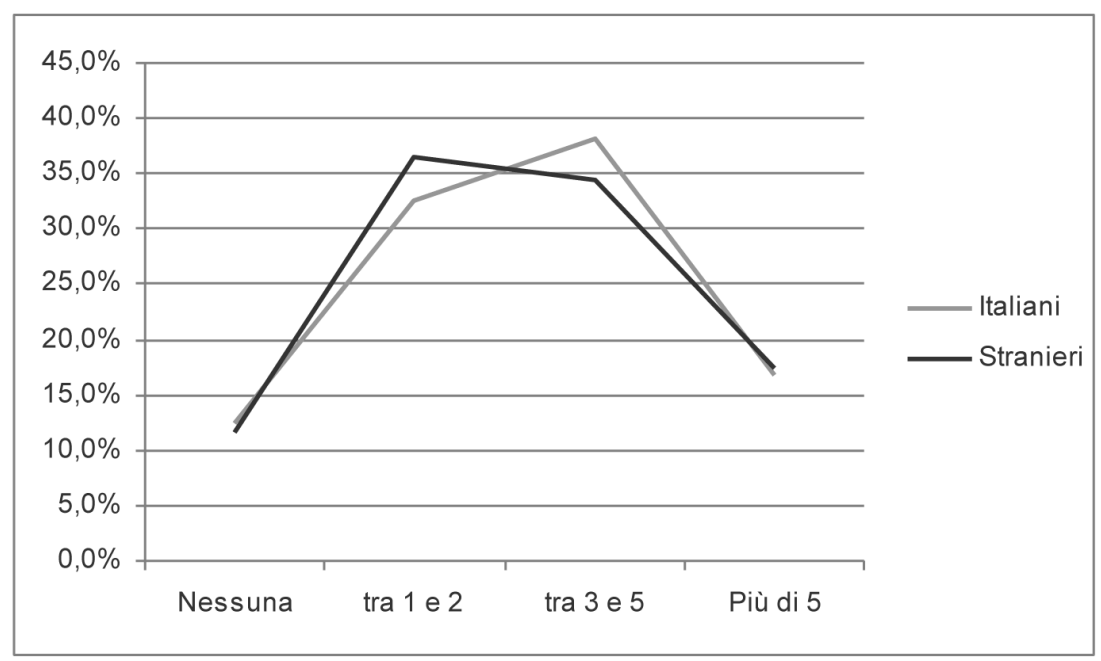

Graf. 2 - Numero di conoscenti: studenti italiani e studenti di cittadinanza non italiana

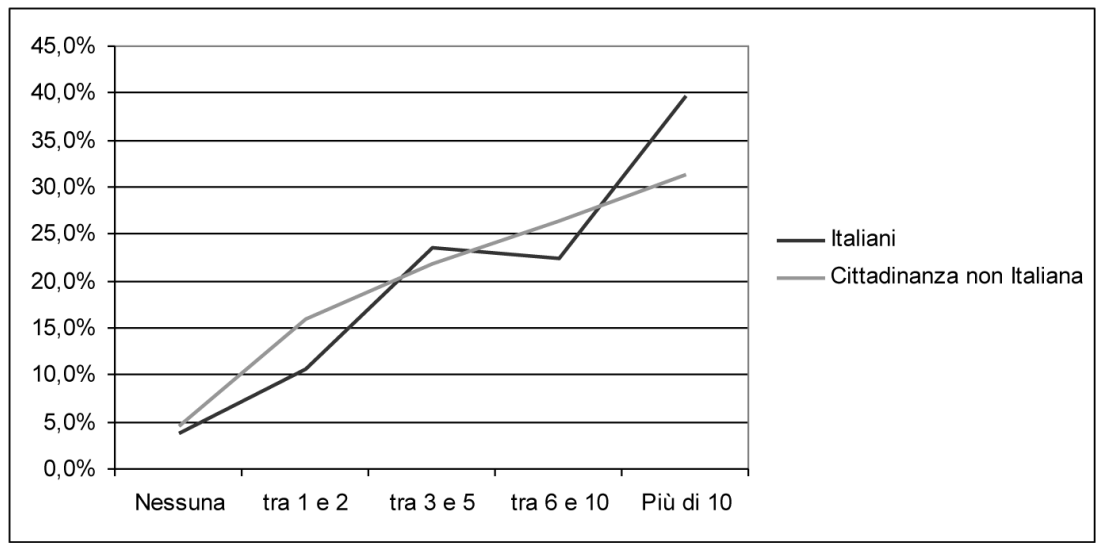




\subsection{Natura delle relazioni e situazione sentimentale}

Per avere maggiori informazioni riguardo alla qualità delle relazioni che gli studenti, italiani e non, creano all'interno dell'università, si è voluto indagare sulla significatività e sull'importanza del rapporto stesso. Quindi ci si è chiesti se i legami di amicizia si creano in base a criteri affettivi (Tab. 4) (trascorrere insieme il tempo libero e confidarsi, condividere i problemi) o in base a criteri funzionali (studiare insieme per raggiungere gli obiettivi accademici e scambiarsi informazioni, materiali, ecc.).

Per entrambi i gruppi di studenti le relazioni all'interno dell'università rispondono principalmente al criterio funzionale (studenti italiani 64,2\%, studenti di cittadinanza non italiana $65,8 \%$ ) piuttosto che al criterio affettivo (studenti italiani 35,8\%, studenti di cittadinanza non italiana 34,2\%).

Tab. 4 - Criterio affettivo e funzionale degli studenti di cittadinanza italiana e non

\begin{tabular}{lcc}
\hline & Studenti italiani & $\begin{array}{c}\text { Studenti di altra } \\
\text { cittadinanza }\end{array}$ \\
\hline $\begin{array}{l}\text { Trascorrere insieme il tempo libero } \\
\text { (cene, uscite serali, cinema, ecc.) }\end{array}$ & $20,70 \%$ & $15,80 \%$ \\
\hline Confidarsi, condividere problemi & $15,10 \%$ & $18,40 \%$ \\
\hline Tot. Criterio affettivo & $35,80 \%$ & $34,20 \%$ \\
\hline $\begin{array}{l}\text { Studiare insieme per raggiungere } \\
\text { gli obiettivi accademici }\end{array}$ & $23,20 \%$ & $27,00 \%$ \\
\hline Scambiarsi informazioni, materiali, ecc. & $41,00 \%$ & $38,80 \%$ \\
\hline Tot. Criterio funzionale & $64,20 \%$ & $65,80 \%$ \\
\hline Totale & $100,00 \%$ & $100,00 \%$ \\
\hline
\end{tabular}

Entrando nello specifico del campione degli studenti di cittadinanza non italiana, tra i due sottogruppi non si riscontrano differenze significative rispetto al criterio privilegiato.

È l'unico caso in cui, nella nostra analisi sulla socialità, gli studenti internazionali seguono lo stesso andamento degli studenti scolarizzati in Italia.

Altro elemento interessante da indagare in relazione alla socialità riguarda la variabile "situazione sentimentale". In questo caso è stato chiesto ai membri dei campioni se avessero o meno una relazione sentimentale. Alla domanda formulata potevano essere date tre risposte: "single", "impegnato" e "non definita" (Tab. 5). Si può affermare che la situazione "non definita" è quella che ha minor incidenza in entrambi i campioni. Fra gli ita- 
liani è molto elevato il numero degli impegnati $(61,2 \%)$, a fronte del $50 \%$ degli studenti di cittadinanza non italiana.

Tab. 5 - Situazione sentimentale degli studenti di cittadinanza italiana e non

\begin{tabular}{lccc}
\hline & Italiani & $\begin{array}{c}\text { Cittadinanza } \\
\text { non italiana }\end{array}$ & Differenze \\
\hline Non definita & $7,8 \%$ & $11,2 \%$ & $-3,4 \%$ \\
\hline Single & $31,0 \%$ & $38,8 \%$ & $-7,8 \%$ \\
\hline Impegnato & $61,2 \%$ & $50,0 \%$ & $11,2 \%$ \\
\hline Totale & $100 \%$ & $100 \%$ & $0 \%$ \\
\hline $\begin{array}{l}\text { Chi Quadro } \\
\text { di Pearson }\end{array}$ & Valore & df & Sig. asint. (2 vie) \\
\hline
\end{tabular}

Per quanto riguarda gli studenti di cittadinanza non italiana si evidenzia una significativa differenza $(0,054$ Chi Quadro) tra i due sottogruppi. In particolar modo sono gli studenti scolarizzati in Italia $(61,4 \%)$ ad avere una maggiore facilità ad intrattenere una relazione sentimentale rispetto agli studenti internazionali $(43,2 \%)$. Si riscontra infatti tra i due sottogruppi una differenza del 18,2\%. Ciò, associato ai dati sul numero di amici e conoscenti, permette di individuare una condizione di maggior solitudine degli studenti internazionali.

\subsection{Le esperienze di associazionismo}

Altro aspetto indagato nella ricerca è stato il grado di appartenenza o inserimento di studenti italiani e non all'interno di associazioni/organizzazioni socio-culturali presenti nella città dell'Aquila, sia all'interno che all'esterno dell'università. Il primo dato significativo è la bassa percentuale di studenti di entrambi i campioni alla partecipazione attiva in associazioni e organizzazioni, percentuale che non supera il $20 \%$. Seppure si tratta di una minoranza, ci sembra rilevante che 1 studente su 5 sia impegnato in attività di associazionismo e volontariato (Graf. 3).

Tra gli studenti italiani e gli studenti di cittadinanza non italiana c'è una leggera differenza percentuale a favore di questi ultimi (2,5\%). Alla luce di quanto riscontrato sarebbe interessante, con studi successivi, accertare se 


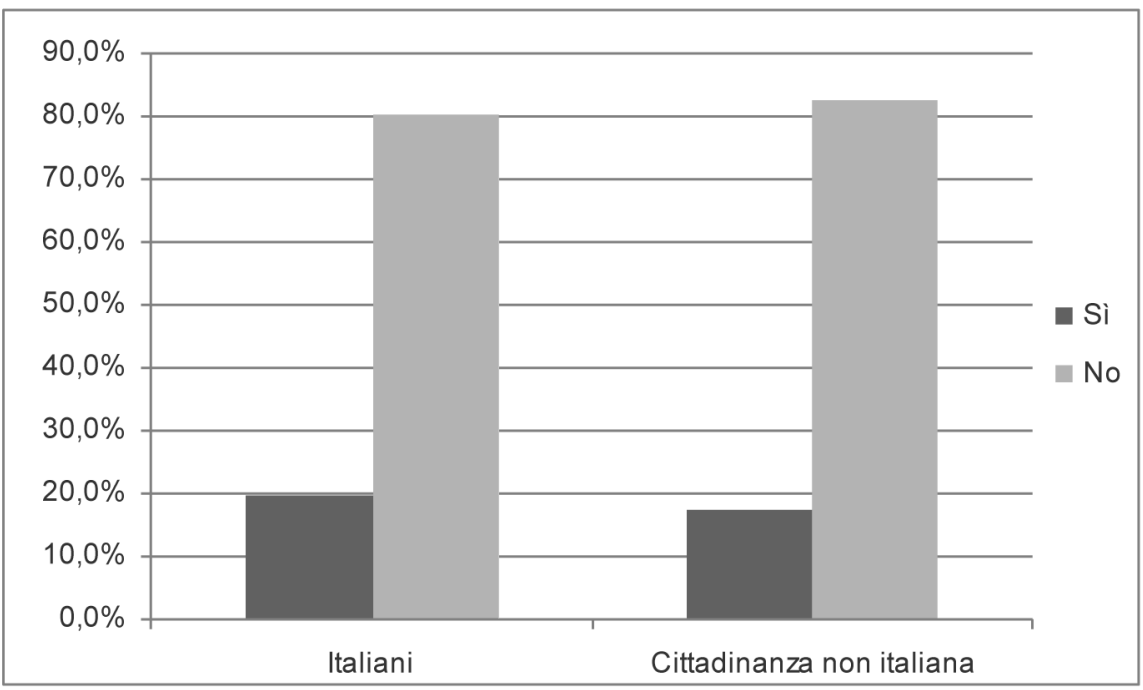

esistono o meno relazioni attive tra le associazioni/organizzazioni socioculturali cittadine e gli studenti universitari.

Le associazioni/organizzazioni di volontariato e culturali sono le più frequentate sia tra gli studenti italiani sia tra gli studenti di cittadinanza non italiana (studenti italiani 49,9\%; studenti di cittadinanza non italiana 73,6\%), seguono quelle sociali e sportive (studenti italiani 13,6\%; studenti di cittadinanza non italiana 21,1\%); infine, con una bassa percentuale (studenti italiani 3\%; studenti di cittadinanza non italiana 5,3\%) quelle di natura religiosa.

Alcune associazioni come nel caso di quelle studentesche sono frequentate esclusivamente dagli studenti italiani.

Avendo a disposizione dati riguardanti la valutazione degli studenti di cittadinanza non italiana rispetto all'attenzione che le organizzazioni studentesche rivolgono alle loro problematiche, si è pensato di indagare su quanto il livello d'attenzione ai problemi legati alla convivenza e all'inclusione, possa influire sulla scelta del singolo di appartenervi o meno. Si è riscontrato che la maggior parte degli studenti di cittadinanza non italiana $(74,5 \%)$ reputa l'operato delle organizzazioni poco affine alle loro problematiche. È pertanto ipotizzabile che la non appartenenza a questo tipo di organizzazione derivi proprio dalla poca attenzione rivolta ai problemi degli stessi studenti di cittadinanza non italiana. Da ciò, ci sembrano maturi i tempi anche per ripensare, in chiave interculturale, il sistema delle rappresentanze studentesche, che coprono un ruolo significativo nei processi decisionali interni agli atenei. 


\title{
12. Chi è l'altro? Percezioni a confronto
}

\author{
di Maria Grazia Ferretti, Roberto Manzi'
}

\subsection{La percezione dell'altro}

Questa parte dell'indagine è tesa ad analizzare e mettere a confronto dati riguardanti alcuni atteggiamenti che gli studenti italiani hanno nei confronti dei colleghi di altra cittadinanza e viceversa.

Prima, però, di addentrarci nell'analisi dei dati ottenuti dall'indagine, pare opportuno sottolineare la peculiarità della stessa, che sta nel carattere relativo e soggettivo legato al concetto di percezione dell'altro.

Lo studio della percezione sociale riguarda in sostanza i modi in cui creiamo impressioni e formuliamo giudizi nei confronti degli altri. Secondo la psicologia sociale studiare il comportamento degli altri ci aiuta a comprendere il nostro mondo sociale.

Ciò che vediamo, sentiamo, percepiamo e inevitabilmente giudichiamo è l'individuo che ci si trova davanti, ed è proprio questa salienza percettiva uno dei fattori che ci fa attribuire ad esso la causa dei comportamenti che osserviamo.

Prima ancora di chiedersi "chi è l'altro" e studiarne il comportamento, ogni individuo deve passare inevitabilmente, attraverso la conoscenza di sé.

Il sé è composto in primo luogo dai nostri pensieri e credenze su noi stessi (concetto di sé). In secondo luogo, il sé è anche un attivo elaboratore di informazioni (consapevolezza o coscienza). Questi due processi psicologici si combinano insieme per creare un senso coerente di identità.

Il punto nodale nel nostro contesto è proprio conoscere noi stessi attraverso la percezione dell'altro e il confronto sociale.

1. I paragrafi 12.1. e 12.2. vanno attribuiti a Roberto Manzi, mentre il paragrafo 12.3. a Maria Grazia Ferretti. 
In tal senso ci può aiutare la teoria del confronto sociale (Festinger, 1954) che verte su due importanti questioni:

- quando procediamo al confronto sociale? Evidentemente quando ci mancano dati oggettivi con cui poterci misurare e siamo in una situazione di incertezza;

- con chi scegliamo di farlo? In un prima fase con chiunque, in seguito ci si confronta con chi è simile a noi, in una dimensione o attributo considerato importante.

La percezione dell'altro, quindi, ci permette di conoscere noi stessi attraverso gli occhi degli altri; spesso, infatti, le altre persone osservano la nostra personalità e le nostre emozioni in modo diverso da noi, e a volte sono in grado di fare valutazioni migliori. Questo perché spesso gli individui possono non voler riconoscere i propri tratti negativi e quindi formulano previsioni errate sul proprio comportamento. Inoltre gran parte del nostro pensiero riguardo al mondo opera in maniera automatica e inconscia, per cui gli altri sono in grado di valutarlo meglio rispetto a noi stessi.

Una corretta percezione dell'altro deve però passare attraverso il superamento di pregiudizi e stereotipi (cfr. Nobile, 2014; Vaccarelli, 2008).

Il pregiudizio è un giudizio precedente all'esperienza o in assenza di dati sufficienti. Esso si definisce come un atteggiamento ostile o negativo nei confronti dei membri di un gruppo, basato solo sull'appartenenza a quel gruppo.

Per la psicologia sociale l'atteggiamento (Allport, 1973) è la posizione, in senso favorevole o contrario, che una persona ha dentro di sé, nei confronti di qualcosa.

Tre sono gli elementi che compongono l'atteggiamento (cfr. Vaccarelli, 2008):

- la componente cognitiva, che comprende le percezioni, i ricordi, i pensieri che riguardano l'oggetto dell'atteggiamento e tutto ciò che vi è strettamente collegato;

- la componente affettiva, nella quale rientrano le reazioni emotive scatenate dalla presenza dell'oggetto o dall'idea di trovarsi in presenza di esso o da cose strettamente correlate;

- la componente conativa, che racchiude ogni tendenza della persona a comportarsi in un dato modo nei confronti dell'oggetto.

Il pregiudizio, quindi, altro non è se non un atteggiamento favorevole o sfavorevole nei confronti di un elemento qualsiasi, che si forma in assenza di un'informazione sufficiente e che resiste alle modificazioni e alle discussioni razionali. Sono state proposte diverse spiegazioni del pregiudizio in chiave psicologico-sociale; Allport (1973) distingue sei ti- 
pi di teorie del pregiudizio in relazione con altrettanti livelli diversi di analisi causale:

- l'approccio storico, che si basa sul principio dello sfruttamento, dove il pregiudizio viene affrontato in quanto modo di legittimazione di vantaggi economici;

- l'approccio socio-culturale, che affronta il pregiudizio a partire dai fattori che riguardano la cultura e la sottocultura;

- l'approccio situazionale, che comprende l'insieme delle forme esterne che agiscono sull'individuo e considera tutti gli effetti della mobilità sociale;

- l'approccio psico-dinamico, che presuppone la centralità del processo di socializzazione e di inculcamento;

- l'approccio fenomenologico, che ha per oggetto il sistema di risposte agli stimoli esterni, risposte conformi alla concezione del mondo propria del soggetto;

- l'approccio sulla base dell'oggetto-stimolo, che parte dalle caratteristiche reali imputabili a diversi gruppi etnici.

\subsection{Le percezioni sull'immigrazione nello scenario na- zionale e internazionale}

Dal 2011 il tema dell'immigrazione è tornato, per l'ennesima volta, in primo piano anche sulla scena politica internazionale, soprattutto sulla scia delle rivoluzioni della primavera araba e il seguente flusso migratorio dai Paesi nordafricani verso le coste italiane.

Secondo i dati raccolti dall'Osservatorio di Pavia, nei primi quattro mesi del 2011, ad esempio, l'immigrazione nei telegiornali italiani ha ricevuto un'attenzione pari al 6\%, contro una media europea del 2\% (Osservatorio di Pavia, 2011).

Sul versante internazionale, l'indagine TTI (Transatlatic Trends, 2010), ha messo in evidenza come gli italiani si mostrino tra i più scettici nei confronti dell'immigrazione. Se nel 2008 più del 50\%, infatti, affermava di ritenere eccessivo il numero di immigrati nel Paese e 1'80\% si diceva preoccupato dall'immigrazione clandestina, nel 2010 la percezione degli immigrati si è ulteriormente inasprita: nel 2009 il 34\% degli italiani riteneva che gli immigrati regolari contribuissero all'aumento della criminalità, mentre nel 2010 la percentuale sale al 56\%, percentuale essenzialmente analoga a quella relativa agli italiani convinti che gli immigrati irregolari concorrano ad aumentare la criminalità (57\%).

Rispetto agli adulti, la diffidenza dei giovani nei confronti degli immigrati sembra essere maggiore, come rileva una ricerca realizzata da GfkEurisco: per circa il $76 \%$ dei giovani intervistati, infatti, la presenza di 
stranieri immigrati nel nostro Paese è ritenuta essere "numerosa" o "molto numerosa", ma solo il $22 \%$ vede in maniera positiva questo fenomeno, a differenza degli adulti, per i quali (71\%), la presenza di stranieri nel nostro Paese è "numerosa" o "molto numerosa", ma il $35 \%$ ne ha un'opinione positiva.

Non sono però tanto le differenze culturali e religiose a intimorire $i$ giovani, quanto l'aumento dell'incertezza quotidiana, dovuto principalmente alla criminalità e alla precarietà del proprio futuro, soprattutto lavorativo. Quello che pare emergere dalle indagini condotte negli ultimi anni è come sia il senso di insicurezza a influire in modo negativo sulla percezione del fenomeno migratorio: l'insicurezza per la propria incolumità, l'insicurezza generata dalla crisi economica e occupazionale, che vede gli immigrati quali pericolosi competitori nell'accesso ai posti di lavoro, e l'insicurezza alimentata dal declino del sistema di welfare (Eurispes, 2011; Gfk-Eurisko, 2010).

L'impressione, segnalata da più parti, è che i processi a favore dell'integrazione in Italia abbiano rallentato la loro dinamicità, laddove prende corpo una certa preoccupazione per l'impatto complessivo di un'immigrazione cresciuta più rapidamente di quanto non ci si aspettasse.

Ma se questo è lo scenario di sfondo, le opinioni espresse dagli intervistati rivelano che esiste una serie di atteggiamenti assai complessi, a seconda dei diversi ambiti che di volta in volta vengono presi in considerazione; è infatti largamente condivisa non solo l'idea che gli immigrati debbano godere dei diritti politici attualmente riservati ai cittadini, ma anche quella che gli stranieri siano un fattore positivo per lo sviluppo dell'economia.

\subsection{Atteggiamenti e percezioni nell'Università dell'Aquila}

Per poter indagare a fondo gli atteggiamenti e la componente pregiudiziale in essi contenuta, la via migliore sarebbe quella di interrogare direttamente gli individui, ma questo è anche il campo più difficile da esplorare e le risposte sono, perlopiù, influenzate dal modo in cui sono poste le domande.

Abbiamo, quindi, fatto ricorso alla tecnica delle scale (Corbetta, 1999), che consiste in un insieme di procedure messe a punto per misurare concetti complessi e non direttamente osservabili.

La tecnica delle scale è usata soprattutto quando l'unità d'analisi è l'individuo, il concetto generale è un atteggiamento (credenze di fondo non rilevabili direttamente) e i concetti specifici sono le opinioni (espressione empiricamente rilevabile di un atteggiamento).

Gli elementi di una scala sono tipicamente domande chiuse che possono essere proposte in modi diversi, per quanto ci riguarda le categorie di risposta sono a parziale autonomia semantica, cioè il significato di ogni ca- 
tegoria è parzialmente autonomo dalle altre ("molto", "abbastanza", "poco", "per nulla").

Si è scelto di utilizzare la scala di Likert (Ibidem), la cui procedura di base consiste nella somma dei punti attribuiti ad ogni singola domanda posta come affermazione, per ognuna delle quali l'intervistato deve dire se e in che misura è d'accordo.

Solitamente le alternative di risposta sono cinque, da "molto d'accordo" a "fortemente contrario"; in questa indagine si è deciso di dare quattro alternative per evitare l'errore della tendenza centrale nelle risposte.

Alla luce di quanto emerge dagli studi europei e nazionali sull'integrazione, è stato interessante mettere in relazione alcuni elementi significativi, al fine di comprendere meglio gli atteggiamenti verso gli immigrati.

Uno di questi elementi è la percentuale di cittadini migranti presenti in Italia percepita dagli studenti italiani, per verificare se esista in qualche modo una sindrome da invasione che sembra essere strisciante nell'intero contesto sociale ${ }^{2}$. Attualmente nel nostro Paese 1'8,1\% della popolazione è di origine straniera; dalla nostra indagine - attraverso una domanda costruita ad hoc - emerge che, in media, secondo gli studenti italiani la popolazione di origine straniera presente in Italia pesi per il $16 \%$ sull'intera popolazione, esattamente il doppio di quello che nei fatti osserviamo. Si tratta di sindrome da invasione? Per rispondere alla domanda analizziamo, per prima cosa, come gli studenti italiani si pongono nei confronti dell'immigrazione in Italia.

Abbiamo fornito sei affermazioni e chiesto di fornire accordo o disaccordo con le stesse (Tab. 1).

Dalle risposte forniteci possiamo dedurre che gli studenti italiani mostrano nella maggior parte dei casi segnali di apertura e di disponibilità nel riconoscere il ruolo dell'immigrazione all'interno della società italiana. In molti i casi, infatti, sembrano in grado di elaborare giudizi autonomi sull'immigrazione in relazione ad ambiti della vita sociale diversi tra loro per contenuto e per impatto sulla quotidianità. Tuttavia esiste una minoranza numericamente significativa, che si attesta intorno al $20 \%$, che nutre atteggiamenti sfavorevoli nei confronti degli immigrati.

2. Nel contestualizzare i dati riportati nei prossimi paragrafi e capitoli, non possiamo non far riferimento a precedenti ricerche svolte nel territorio aquilano dopo il sisma del 2009, che hanno evidenziato come le dinamiche dei rapporti e delle percezioni tra italiani e stranieri abbiano risentito delle più generali situazioni di stress sociale: nel dopo terremoto si sono fatti avanti infatti sentimenti di competizione relativi alle emergenze abitative, una percezione della presenza di immigrati decisamente sovrastimata, un rischio di conflitto sociale, nelle fasce più disagiate della popolazione, legato alla classica dinamica del capro espiatorio (Vaccarelli, 2010; 2011). 
Tab. 1 - Gli atteggiamenti nei confronti degli immigrati (studenti italiani)

\begin{tabular}{|c|c|c|c|c|}
\hline & $\begin{array}{c}\text { Molto } \\
\text { d'accordo }\end{array}$ & $\begin{array}{l}\text { Abbastanza } \\
\text { d'accordo }\end{array}$ & $\begin{array}{l}\text { Abbastanza } \\
\text { contrario }\end{array}$ & $\begin{array}{l}\text { Molto } \\
\text { contrario }\end{array}$ \\
\hline $\begin{array}{l}\text { La presenza di stranieri } \\
\text { in Italia è positiva perché } \\
\text { stimola il confronto tra la } \\
\text { nostra cultura e le altre }\end{array}$ & $32,0 \%$ & $50,3 \%$ & $14,4 \%$ & $3,4 \%$ \\
\hline $\begin{array}{l}\text { Gli stranieri danno un utile } \\
\text { contributo all'economia del } \\
\text { territorio }\end{array}$ & $19,6 \%$ & $47,2 \%$ & $25,8 \%$ & $7,5 \%$ \\
\hline $\begin{array}{l}\text { Gli stranieri sono persone } \\
\text { che cercano soltanto di } \\
\text { migliorare la loro condizione } \\
\text { di vita senza recar danno a } \\
\text { nessuno }\end{array}$ & $18,6 \%$ & $55,8 \%$ & $19,9 \%$ & $5,7 \%$ \\
\hline $\begin{array}{l}\text { Le amministrazioni locali } \\
\text { devono essere più attente } \\
\text { alle problematiche degli } \\
\text { stranieri per favorire la loro } \\
\text { integrazione }\end{array}$ & $29,5 \%$ & $47,7 \%$ & $18,7 \%$ & $4,1 \%$ \\
\hline $\begin{array}{l}\text { Gli stranieri portano via il } \\
\text { lavoro e le case agli italiani }\end{array}$ & $3,6 \%$ & $14,5 \%$ & $43,0 \%$ & $38,9 \%$ \\
\hline $\begin{array}{l}\text { Gli stranieri portano degrado } \\
\text { nei luoghi in cui viviamo }\end{array}$ & $4,1 \%$ & $16,0 \%$ & $43,2 \%$ & $36,7 \%$ \\
\hline
\end{tabular}

È interessante notare, inoltre, come al crescere di una percezione distorta della presenza di migranti in Italia, gli atteggiamenti nei loro confronti siano meno favorevoli: incrociando le variabili (indice di atteggiamento e stima percentuale della presenza straniera in Italia) si rileva infatti una correlazione negativa $-0,2$ con significatività, 004 . Chi tende a sovrastimare la presenza di stranieri in Italia percepisce, poi, anche un maggior numero di stranieri presenti all'università, con una correlazione di 0,6 , dunque piuttosto forte, e significatività, 000 .

A fronte di una percezione della presenza degli stranieri in Italia e nell'Università dell'Aquila sovrastimata, associata ad atteggiamenti di competizione o di allarme soprattutto legati al lavoro, al degrado sociale, alle politiche sociali, possiamo ragionevolmente pensare che i tratti tipici della cosiddetta sindrome d'assedio riguardino almeno 1 studente italiano su 5, fatto che non possiamo considerare trascurabile, trattandosi di soggetti ben scolarizzati e prossimi all'acquisizione di una laurea.

Per comprendere quale sia la percezione dell'altro nei soggetti intervistati abbiamo posto diversi quesiti, tra i quali quello riguardante l'idea che 
gli studenti italiani hanno dei propri colleghi universitari di diversa cittadinanza e viceversa.

Sono state fornite 14 affermazioni sulle quali gli intervistati hanno espresso accordo o disaccordo (scala da 1 a 4).

La Tabella 2 che segue riporta le medie ottenute sui valori rapportati ad una scala 0-10 per rendere il dato più facilmente leggibile. Sulla colonna destra, per motivi di sintesi e di leggibilità, si segnala con un sì o con un no

Tab. 2 - Atteggiamenti reciproci: studenti italiani e studenti non italiani

\begin{tabular}{|c|c|c|c|}
\hline & $\begin{array}{c}\text { Non italiani } \\
\text { vs } \\
\text { italiani }\end{array}$ & $\begin{array}{c}\text { Italiani } \\
\text { vs } \\
\text { non italiani }\end{array}$ & $\begin{array}{l}\text { Analisi della } \\
\text { Varianza con } F \\
\text { significativo }\end{array}$ \\
\hline & Media & Media & \\
\hline $\begin{array}{l}\text { Sono disponibili al confronto e } \\
\text { all'aiuto }\end{array}$ & 6,6 & 6,4 & No \\
\hline $\begin{array}{l}\text { Sono aperti e curiosi nei confronti } \\
\text { delle altre culture }\end{array}$ & 4,9 & 6,4 & Sì \\
\hline $\begin{array}{l}\text { Sanno condividere le esperienze } \\
\text { personali }\end{array}$ & 5,0 & 6,0 & Sì (lieve) \\
\hline Sono sensibili ai problemi degli altri & 4,7 & 5,9 & Sì \\
\hline $\begin{array}{l}\text { Hanno sensibilità per i problemi } \\
\text { sociali }\end{array}$ & 4,6 & 5,6 & Sì \\
\hline Sono disponibili all'amicizia & 5,5 & 6,7 & Sì \\
\hline $\begin{array}{l}\text { Sanno condividere le esperienze } \\
\text { nello studio }\end{array}$ & 5,4 & 6,0 & No \\
\hline $\begin{array}{l}\text { Si impegnano per ottenere buoni } \\
\text { risultati accademici }\end{array}$ & 6,1 & 6,7 & No \\
\hline $\begin{array}{l}\text { Partecipano attivamente alle } \\
\text { iniziative di carattere sociale }\end{array}$ & 4,1 & 5,0 & No \\
\hline $\begin{array}{l}\text { Partecipano attivamente alle } \\
\text { iniziative per il miglioramento } \\
\text { della realtà universitaria }\end{array}$ & 3,7 & 4,4 & No \\
\hline $\begin{array}{l}\text { Partecipano attivamente alle } \\
\text { iniziative per il miglioramento } \\
\text { della realtà universitaria }\end{array}$ & 3,7 & 4,4 & No \\
\hline Rispettano le diversità culturali & 5,5 & 6,4 & No \\
\hline Rispettano le diversità religiose & 5,7 & 6,3 & No \\
\hline $\begin{array}{l}\text { Accettano di buon grado amici } \\
\text { italiani/stranieri }\end{array}$ & 5,5 & 6,9 & Sì \\
\hline Sono solidali & 5,3 & 6,4 & Sì (lieve) \\
\hline Indice Atteggiamento & 5,2 & 5,9 & Sì \\
\hline
\end{tabular}


l'esistenza di una significatività statistica (associata all'indice F in applicazione dell'analisi della varianza) tale da far considerare le differenze come effettivamente rilevanti.

Dal confronto tra le risposte date dai due campioni risulta che gli studenti italiani tendono ad avere complessivamente un'idea più positiva dei colleghi di altra cittadinanza, rispetto a quella che questi ultimi hanno dei colleghi italiani. Nello specifico, gli studenti italiani vedono gli studenti di altra cittadinanza maggiormente aperti e curiosi nei confronti delle altre culture, maggiormente aperti alla condivisione delle esperienze, più sensibili ai problemi degli altri e ai problemi sociali, più solidali e disponibili alle amicizie con persone di altra cittadinanza, di quanto gli studenti di origine estera percepiscano gli studenti italiani. Questo fatto può dipendere da un punto di vista diverso e probabilmente maggiormente caratterizzato, per quanto riguarda gli studenti di cittadinanza non italiana, non solo da bisogni di socializzazione più marcati (e dunque da aspettative diverse da chi sicuramente ha già, anche al di fuori del contesto universitario, una rete sociale) ma anche da esperienze di discriminazione che, come vedremo nel prossimo capitolo, colpiscono molti ragazzi di origine estera.

Un approfondimento dei dati - anche rispetto alle percezioni che ogni gruppo dà di se stesso - lo possiamo considerare in rapporto ai valori percentuali ottenuti sui diversi item, facendo riferimento alle tabelle presenti negli Allegati 1 e 2 del volume. Dai dati emerge la tendenza di entrambi i gruppi a posizionarsi sul valore "abbastanza d'accordo" rispetto a tutte le affermazioni sulle quali si è chiesto di dare un'opinione, ad esclusione di quella riguardante la partecipazione sociale, riguardo alla quale sia italiani, sia studenti con altra cittadinanza si posizionano in maniera simile su "abbastanza d'accordo" (46,4\% italiani, 39,1\% altra cittadinanza) e "poco d'accordo" (41,3\% italiani, $44,4 \%$ altra cittadinanza).

Stessa cosa accade con l'affermazione relativa alla partecipazione attiva alle iniziative per il miglioramento della realtà universitaria, con una prevalenza del "poco d'accordo" (47,3\% italiani, 51,7\% altra cittadinanza) sull'"abbastanza" (36,6\% italiani, $31,8 \%$ altra cittadinanza) in entrambi i gruppi e in modo più evidente nel gruppo degli studenti con altra cittadinanza.

Le stesse affermazioni sono state presentate anche in relazione a ciò che si pensa dei colleghi universitari italiani ed anche qui emerge che, sia per gli studenti di altra cittadinanza, che per gli italiani, c'è l'idea che gli studenti italiani siano poco predisposti alla partecipazione sociale $(60,5 \%$ italiani, $50 \%$ altra cittadinanza) e poco inclini ad impegnarsi nel miglioramento della realtà universitaria (57\% italiani, 52,6\% altra cittadinanza).

Quanto emerso ci porta a constatare che gli studenti percepiscono poca inclinazione a partecipare attivamente a iniziative di carattere sociale 
e quasi disinteresse per l'ambiente universitario, pur essendo quest'ultimo l'ambiente da loro maggiormente frequentato.

Analizzando, poi, gli atteggiamenti in base alla provenienza geografica degli studenti di cittadinanza non italiana, non emergono associazioni significative, così come non si riscontrano differenze significative tra studenti internazionali e studenti stranieri scolarizzati in Italia.

Notiamo un atteggiamento in controtendenza, anche se lievemente significativo, negli studenti stranieri con un indice di status socio-economico meno elevato; nello specifico, all'abbassarsi di tale indice corrisponde una percezione più negativa dei colleghi italiani (correlazione $-0,15$, significatività 0,03 ) e anche della cultura italiana in generale (correlazione $-0,167$, significatività 0,03$)$.

Possiamo, quindi, ipotizzare che ci sia un sentimento di competizione a livello sociale negli studenti non italiani e, probabilmente, la sensazione di essere poco tutelati e rappresentati da parte del sistema socio-politico italiano.

Un altro quesito preso in considerazione è quello che riguarda l'idea che gli studenti - italiani e di altra cittadinanza - hanno dell'Italia. La domanda non ha preso in considerazione gli atteggiamenti ma ha posto alcuni aspetti della cultura italiana da valutare con una scala da 1 a 5. Nella Tabella 3 si riportano i valori rapportati su scala 0-10 per ragioni di maggiore leggibilità e, anche qui, notiamo una significatività statistica indicata sinteticamente con un sì, laddove le differenze sono piuttosto rilevanti.

In questo caso otteniamo un dato alquanto interessante: gli studenti di altra cittadinanza hanno una visione in generale più positiva della cultura italiana rispetto a quella che hanno gli italiani; più nello specifico, analizzando le percentuali di riferimento nelle tabelle degli Allegati 1 e 2 dell'indagine, i valori che emergono sono medio-alti rispetto agli aspetti culturali (ad eccezione dei programmi televisivi con valore 2 per il 26\% e valore 3 per il 31,3\%), al sistema sanitario, al sistema di istruzione e al territorio.

C'è una valutazione più che positiva relativamente alle abitudini alimentari (32,5\% valore $4,39,1 \%$ valore 5), mentre emergono valori tendenzialmente bassi quando si parla di sistema politico e socio-economico e quando si affronta il tema dell'apertura verso altre culture e religioni.

Nonostante la poca considerazione del sistema economico italiano, è interessante constatare come gli studenti non italiani lo valutino in maniera nettamente più positiva rispetto ai colleghi italiani, come accade anche per il sistema sanitario.

Questo ci fa ipotizzare che tra gli studenti italiani ci sia un senso di insoddisfazione maggiore nei confronti del sistema, che risponde probabilmente al particolare momento di crisi generale del nostro Paese. 
Tab. 3 - La percezione della cultura italiana: studenti di cittadinanza non italiana e studenti italiani - Scala 0-10

\begin{tabular}{|c|c|c|c|}
\hline & $\begin{array}{c}\text { Studenti di } \\
\text { cittadinanza non } \\
\text { italiana }\end{array}$ & $\begin{array}{c}\text { Studenti } \\
\text { italiani }\end{array}$ & $\begin{array}{c}\text { Analisi della Varianza } \\
\text { con F significativo }\end{array}$ \\
\hline & Media & Media & \\
\hline Abitudini alimentari & 7,4 & 7,3 & No \\
\hline Istruzione & 5,2 & 4,4 & Sì \\
\hline Apertura culturale & 4,7 & 4,2 & Sì \\
\hline Musica & 6,5 & 5,8 & Sì \\
\hline Cinema e teatro & 6,2 & 5,7 & Sì \\
\hline Programmi TV & 4,6 & 3,8 & Sì \\
\hline Economia & 4,1 & 2,6 & Sì \\
\hline Sport & 6,6 & 5,7 & Sì \\
\hline Politica & 2,6 & 1,7 & Sì (lieve) \\
\hline Sanità & 5,5 & 3,6 & Sì \\
\hline Territorio-ambiente & 5,8 & 4,3 & Sì \\
\hline Indice cultura italiana & 5,3 & 4,4 & Sì \\
\hline
\end{tabular}

Un ulteriore dato significativo è riscontrabile mettendo a confronto l'idea che gli studenti di cittadinanza non italiana hanno dei colleghi italiani e l'indice di qualità dei servizi offerti dall'università; infatti, quanto più positiva è la considerazione che i primi hanno nei confronti dei secondi, tanto più alto è il gradimento rispetto ai servizi che l'università offre (correlazione 0,7 con significatività ,000).

Un altro elemento interessante emerge mettendo in relazione l'età della popolazione studentesca italiana con gli atteggiamenti nei confronti dei colleghi di altra nazionalità: notiamo rispettivamente una lieve correlazione negativa di $-0,14$ con significatività, 004 e di $-0,14$ con significatività ,006; ciò vuol dire che al calare dell'età degli studenti italiani, tende ad abbassarsi anche la stima nei confronti dei colleghi sia italiani che di altra cittadinanza.

Abbiamo, inoltre, una relazione significativa (correlazione 0,22 con significatività ,000) tra l'atteggiamento degli studenti italiani nei confronti dell'immigrazione e gli atteggiamenti verso i colleghi universitari di altra cittadinanza, che ci consente di affermare che la stima nei confronti di questi ultimi cresce all'aumentare dell'apprezzamento della presenza di cittadini stranieri in Italia. 


\title{
13. Pregiudizi e socialità in ambito universitario
}

\author{
di Alessandro Vaccarelli
}

\subsection{Il pregiudizio e i "contesti" della ricerca}

I principali studi sul pregiudizio ne forniscono una definizione generalmente piuttosto accettata, anche se le prospettive disciplinari diverse - in primis quelle della psicologia sociale, della sociologia, della pedagogia interculturale (cfr. Genovese, 2003; Nobile, 2014; Vaccarelli, 2008) - declinano il problema in ambiti differenti e tengono conto delle loro epistemologie specifiche, per quanto spesso trasversali. Il pregiudizio, in senso lato inteso, implica la formulazione di un giudizio che precede l'esperienza, o che procede a partire dalla generalizzazione di un'esperienza limitata e poco rappresentativa; esso può variare per valenza (positiva o negativa) e intensità. È merito della psicologia sociale quello di aver inquadrato, nell'ottica di una definizione più ristretta, anche quegli ambiti (cognitivi, affettivi, comportamentali) in cui l'atteggiamento pregiudiziale si esprime: l'idea stereotipata e preconcetta, interrelata dunque al sentimento che l'oggetto del pregiudizio suscita (rabbia? disprezzo? sentimento di pena? simpatia? antipatia?) e alla reazione comportamentale che spinge verso il tipo e il grado di discriminazione, vale a dire quei comportamenti agiti che già Allport (1973 ha molto significativamente classificato, nelle loro forme più generali, all'interno di una scala che consente di graduare le conseguenze del pregiudizio stesso' ${ }^{1}$.

1. Nel suo testo ormai classico, La natura del pregiudizio, Allport definisce il pregiudizio come «un'antipatia fondata su una generalizzazione falsa e inflessibile», sentita internamente o espressa in modo esplicito. Esso «può essere diretto verso un gruppo nel suo complesso o verso un individuo in quanto membro di quel gruppo» (Allport, 1973, p. 9). I comportamenti che il pregiudizio può attivare sono disposti da Allport lungo una scala gerarchica: offese verbali, barzellette con stereotipi più o meno offensivi - freddezza, distacco, separazione nei rapporti - discriminazione nei lavori, a scuola, sui mezzi di trasporto violenze fisiche, stato di tensione acuta - sterminio, genocidio (Ibidem). 
I dati che presentiamo meritano una necessaria premessa, riferita sia al contesto particolare e generale entro cui la ricerca si è mossa, sia alle scelte metodologiche. Anticipiamo che sono molti gli studenti, italiani e non, che riferiscono atti di discriminazione o la presenza di pregiudizi nell'ambito della loro esperienza di studio all'Aquila. Il caso "L'Aquila" non ci sembra essere tuttavia un "caso": esiste un contesto generale all'interno del quale i dati stessi si spiegano. Sono sotto i nostri occhi, a livello nazionale ed europeo, anche sull'onda di una crisi cavalcata dagli "imprenditori della paura" ${ }^{2}$, i segnali di un'amplificazione dei sentimenti di ostilità e dei comportamenti discriminatori legati alla competizione in campo socio-economico, ma anche alla più generale indisponibilità alla conoscenza o all'accettazione delle diversità. Senza entrare nel merito, si rimanda a recenti ricerche (Lunaria, 2014), che attraverso rilevazioni su casi di effettiva e segnalata discriminazione etnico razziale, evidenziano come la loro distribuzione sul territorio nazionale segua sostanzialmente l'incidenza della presenza straniera sul territorio: il Nord Italia (con quasi il 55\% delle segnalazioni), il Centro (con circa il 34\%), il Sud (con circa l'11\%). Allo stesso modo, la crescita del numero di discriminazioni agite da attori istituzionali è un segnale piuttosto allarmante (Ibidem). In questo senso, piuttosto che immaginare quello dell'Università dell'Aquila come un caso isolato, possiamo pensare che esso rappresenti più la regola che l'eccezione. La speranza è dunque quella di poter suscitare, rispetto al problema affrontato, non solo una riflessione e un dibattito per così dire interni, ma anche una spinta verso ricerche in altri contesti accademici e territoriali.

Da un punto di vista metodologico, va precisato che il termine "pregiudizio" è stato utilizzato nei due questionari come termine di "senso comune" e come parola-stimolo e dunque in un significato molto ampio, che include atteggiamenti o comportamenti diversamente discriminatori. I questionari hanno consentito di esplorare tale dimensione solo ed esclusivamente all'interno del contesto accademico, e dunque non si è sondata la presenza di pregiudizio all'interno delle relazioni sociali extra-accademiche, che si estendono dunque al rapporto con la città e che implicano questioni, come quella abitativa, per le quali sono state riferite, in più occasioni informali, situazioni di discriminazione ${ }^{3}$.

2. Si prende in prestito l'espressione "imprenditori della paura" da Galissot (Galissot, Kilani e Rivera, 2001).

3. Non va dimenticato che L'Aquila, a seguito del sisma del 2009, ancora non esce da un'emergenza abitativa, che ha fatto spesso attivare dinamiche di competizione tra popolazione residente di cittadinanza italiana e non (Vaccarelli, 2010; 2012). 


\subsection{La presenza del pregiudizio secondo gli studenti italiani e di altra cittadinanza}

I dati relativi al pregiudizio sono stati sondati, nei rispettivi questionari, attraverso due domande diverse, che tuttavia possono essere assimilate nell'ottica del confronto e della comparazione. In un caso, per gli studenti di cittadinanza non italiana, si è chiesto di riferire eventuali comportamenti che li hanno in qualche modo fatti sentire discriminati ("Ti sei mai sentito colpito da forme di pregiudizio nell'ambiente accademico?’); nell'altro caso, per gli studenti italiani, si è cercato di rilevare, nella loro percezione, forme di pregiudizio nei confronti dei loro colleghi di altra cittadinanza ("Pensi che ci siano forme di pregiudizio nei confronti degli studenti stranieri nell'ambiente accademico?"). Il Grafico 1 permette di analizzare i dati su più direzioni.

Graf. 1 - La percezione del pregiudizio negli studenti italiani e l'esperienza del pregiudizio negli studenti di altra cittadinanza

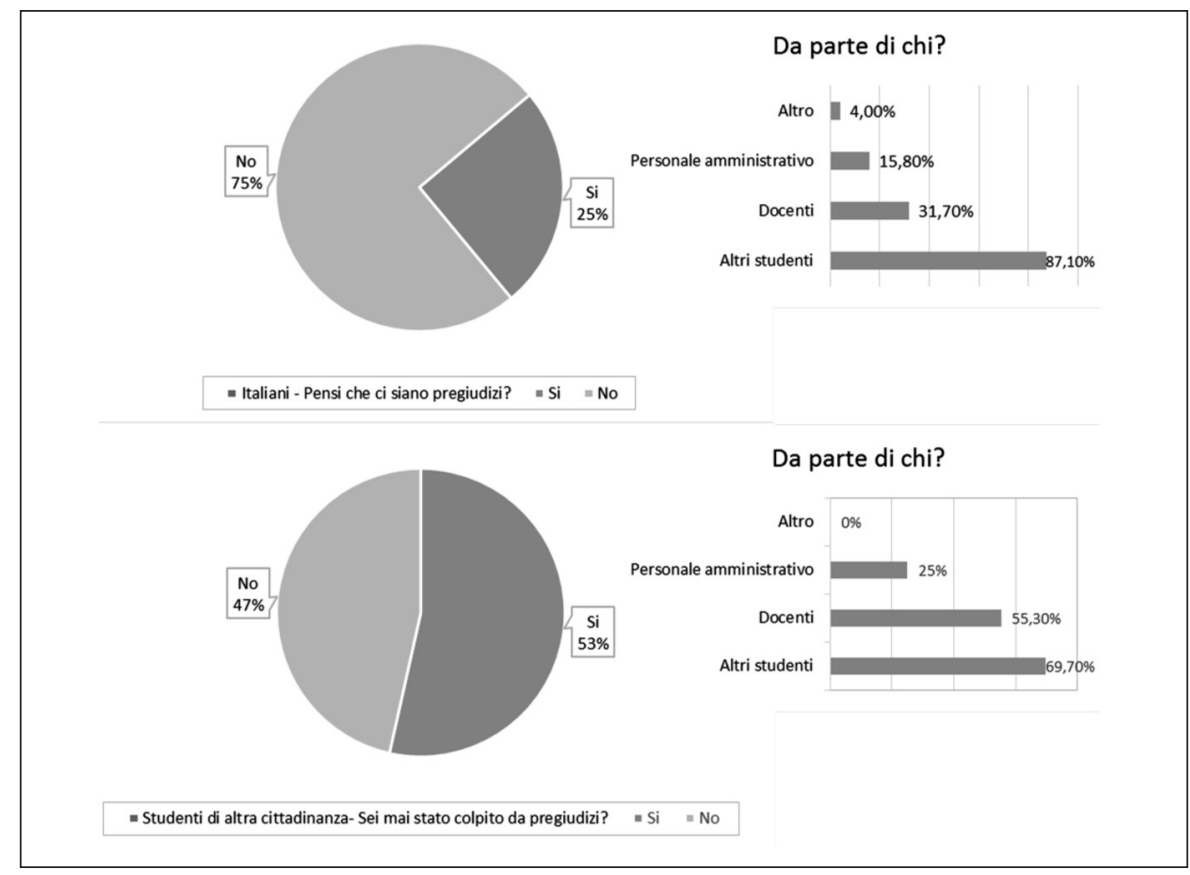

La prima considerazione riguarda l'alto numero di soggetti di cittadinanza non italiana che si sono sentiti colpiti da forme di pregiudizio: il 53,5\% di 
coloro che hanno risposto alla domanda. La seconda considerazione rimanda alla percezione degli studenti italiani, che sembrerebbero sottostimare il fenomeno indagato, con un $25,1 \%$ che risponde affermativamente. Entriamo nel dettaglio indagando anche quali sono i soggetti che gli studenti individuano come principali attori delle dinamiche di discriminazione. I rispondenti di cittadinanza non italiana si sono sentiti colpiti da pregiudizi da parte di altri studenti $(69,7 \%)$, da docenti $(53,3 \%)$, da dipendenti delle strutture amministrative (25\%). Come si deduce dai valori percentuali, prevedendo la domanda la possibilità di fornire risposte multiple, non mancano casi di soggetti che si sono sentiti discriminati da più parti. Tra gli stranieri, sono 28 i soggetti che rispondono alla domanda aperta riferita all'esperienza o alle esperienze di discriminazione (il 18,9\% del campione considerato). Queste possono essere riferite almeno a tre categorie diverse: 1) discriminazioni legate all'uso della lingua (6 risposte); 2) discriminazioni collegate ad atteggiamenti di chiusura, a sentimenti di competizione, a comportamenti denigratori (10 risposte). In questo caso ricorre il tema del numero dei posti riservati agli stranieri nei corsi di laurea a numero programmato; 3) affermazioni generiche su pregiudizi di carattere etnico o religioso (12 risposte). La Tabella 1 riporta a titolo esemplificativo alcune risposte fornite.

I rispondenti italiani testimoniano invece la presenza di comportamenti legati al pregiudizio riferendoli ad altri studenti $(87,1 \%)$, alla componente docenti $(31,7 \%)$, a quella del personale tecnico-amministrativo $(15,8 \%)$. Se troviamo una relativa sottovalutazione del problema e se troviamo anche una minore tendenza a dare testimonianza attraverso le risposte aperte, è interessante notare ciò che generalmente gli studenti italiani rispondono se sollecitati a chiarire le loro posizioni. Accanto a testimonianze che si allineano a quelle degli studenti stranieri, e che dunque implicano una sensibilità di tipo interculturale $(10)^{4}$, troviamo anche posizioni che segnalano la presenza di pregiudizi di tipo positivo riferite ad un eccessivo "buonismo" da parte dei professori (2) o che, addirittura, riferiscono direttamente atteggiamenti ostili di cui i rispondenti sono portatori $(2)^{5}$.

4. «Molti professori riservano agli studenti stranieri un trattamento diverso dagli italiani, alcuni in positivo (accettare risposte vaghe e insufficienti lasciando superare l'esame) e altri in negativo (partendo prevenuti in sede d'esame e apostrofando gli studenti stranieri in modo offensivo)»; «subito dopo il test di ingresso ricordo molti discorsi a proposito dei posti riservati a studenti extracomunitari»; «Molti dei miei colleghi sono soliti parlare male degli stranieri, in maniera razzista e discriminatoria».

5. «Quasi sempre i ragazzi stranieri si presentano assolutamente impreparati agli esami, per cui fanno arrabbiare i professori e fanno perdere tempo agli studenti intenzionati a sostenere l'esame. Il pregiudizio è dello studente serio che si preoccupa perché rischia di dover subire il nervosismo di un professore (giustificato, perché spesso gli stessi studenti, oltre che essere impreparati, sono spocchiosi e irrispettosi) durante l'esame»; «Precedenza agli italiani!». 
1. Discriminazioni legate all'uso della lingua

- Un giorno non ho capito un concetto e ho chiesto al prof. gentilmente di ripeterlo e mi ha risposto che questo è un problema mio che non capisco le lezione perché non capisco l'italiano.

- Posso dare un esempio sì, tra tanti. Sono andata a chiedere a una professoressa se, essendo straniera, avevo diritto a l'uso di un dizionario per l'esame. Non mi ha fatta finire la mia frase che mi rispondeva, a meta urlando e davanti a tutti: "Se lei non parla italiano non capisco perché le hanno dato la possibilità di fare l'inscrizione all'università qui". lo parlo italiano, e penso anche bene. Però non mi sembra un drama se non capisco alcune parole... Ma in generale, i problemi cosi si incontrano più fuori dall'università...

- Durante un esame il docente mi risponde così: "ti metterei 30, ma non mi piace il tuo accento quindi ti metto 29".

2. Discriminazioni riferite ad atteggiamenti di chiusura, a sentimenti di competizione, a comportamenti denigratori

- Mi hanno detto "Albanese di merda" facendolo passare come una frase scherzosa. Mi prendono in giro imitando il mio accento da straniero. In entrambi i casi nessuno è mai intervenuto per rimproverarli, anzi qualcuno sorride pure.

- Alcuni pensano che gli studenti stranieri prendono i posti degli studenti italiani all'università.

- Fino oggi tanti colleghi non parlano con me e non ci provano manco de avvicinarsi a me, per fatto di essere nero

- In sede di esame (dicitura omessa per ragioni di privacy) venivo invitato dal professore di lasciare il Paese ancora prima di iniziare la prova di esame, addirittura mi avvicinava durante le prove scritte e mi diceva "inutile continuare, tanto l'esame con me non lo farai..." passarono ben tre anni e mezzo di poter sostene l'esame con un professore diverso, dovetti attendere il suo trasferimento!!! ASSURDO

3. Affermazioni generiche su pregiudizi di carattere etnico o religioso

- Pregiudizi del tipo: perché stai studiando da noi? perché da voi state dietro anche al livello istituzionale?

- Per la religione praticata / Paese d'origine.

\subsection{II pregiudizio tra gli studenti internazionali e gli stu- denti stranieri scolarizzati in Italia e la relazione con altre variabili}

La percentuale di coloro che riferiscono di essere stati colpiti da pregiudizio si distribuisce tra maschi e femmine con una lieve differenza percentuale (nel sottogruppo delle femmine troviamo un 57,4\% di risposte affermative, a fronte del $52,7 \%$ fornite dai maschi), che tuttavia attraverso il test esatto di Fisher non può essere considerata statisticamente significativa (sig. 0,319). 
All'interno del campione degli studenti di cittadinanza non italiana, troviamo un diverso comportamento della variabile "pregiudizio" se considerata in rapporto agli studenti internazionali e agli studenti stranieri scolarizzati in Italia. Osserviamo infatti che ad essere maggiormente esposti a forme di discriminazione e di pregiudizio sono gli studenti internazionali $(58,9 \%)$ più che gli studenti stranieri scolarizzati in Italia $(47,4 \%)$, con una differenza percentuale pari all'11,5\%. Ciò si spiega a partire dalla concentrazione di tutti gli studenti africani all'interno del primo gruppo, studenti che in moltissimi casi, come vediamo anche nella Tabella 2, riferiscono di essere stati esposti a forme di pregiudizio o discriminazione.

Con la Tabella 2 troviamo infatti che il pregiudizio si distribuisce sull'intero campione di studenti di cittadinanza non italiana - diversamente rispetto alla provenienza geografica, con un Chi Quadro sufficientemente significativo (sig. 0,038).

Tab. 2 - Pregiudizi/discriminazioni per provenienza geografica

\begin{tabular}{|c|c|c|c|c|c|c|}
\hline & \multicolumn{2}{|c|}{ Si } & \multicolumn{2}{|c|}{ No } & \multicolumn{2}{|c|}{ Totale } \\
\hline & v.a. & $\%$ & v.a. & $\%$ & v.a. & $\%$ \\
\hline Africa subsahariana & 16 & $80,0 \%$ & 4 & $20,0 \%$ & 20 & $100,0 \%$ \\
\hline Unione Europea & 28 & $57,1 \%$ & 21 & $42,9 \%$ & 49 & $100,0 \%$ \\
\hline America Latina & 5 & $55,6 \%$ & 4 & $44,4 \%$ & 9 & $100,0 \%$ \\
\hline Medio Oriente & 7 & $53,8 \%$ & 6 & $46,2 \%$ & 13 & $100,0 \%$ \\
\hline Nord Africa & 3 & $50,0 \%$ & 3 & $50,0 \%$ & 6 & $100,0 \%$ \\
\hline $\begin{array}{l}\text { Europa balcanica e } \\
\text { orientale }\end{array}$ & 18 & $47,4 \%$ & 20 & $52,6 \%$ & 38 & $100,0 \%$ \\
\hline Asia & 2 & $14,3 \%$ & 12 & $85,7 \%$ & 14 & $100,0 \%$ \\
\hline $\begin{array}{l}\text { Europa mediterranea } \\
\text { (Cipro) }\end{array}$ & 1 & & 0 & & 1 & \\
\hline Nord America & 1 & & 0 & & 1 & \\
\hline $\begin{array}{l}\text { Cittadinanza non } \\
\text { indicata }\end{array}$ & 1 & & 0 & & 1 & \\
\hline \multirow[t]{2}{*}{ Totale } & 82 & $53,9 \%$ & 70 & $46,1 \%$ & 152 & $100,0 \%$ \\
\hline & & $\begin{array}{l}\text { Valore } \\
17800^{a}\end{array}$ & 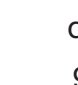 & & asint & vie) \\
\hline
\end{tabular}

Se troviamo una situazione tendenzialmente omogenea rispetto alle provenienze geografiche legate alle realtà europee, mediorientali, nordafricane, latinoamericane, osserviamo che gli studenti provenienti dall'A- 
frica subsahariana riferiscono di aver subito discriminazioni addirittura nell' $80 \%$ dei casi (16 sì, 4 no). Nel caso dei Paesi europei, abbiamo osservato che, nell'ordine, si sentono maggiormente colpiti da pregiudizi gli studenti greci $(65,2 \%)$, quelli albanesi e macedoni (in entrambi i casi per il $50, \%)$, gli studenti rumeni $(43,7 \%)$.

Viceversa, gli asiatici sono coloro che segnalano una più ridotta frequenza ( 2 sì a fronte di 12 no). Ciò lascia ipotizzare che ad accompagnare le forme di pregiudizio ci siano retaggi legati alla percezione e alla "significazione" delle caratteristiche somatiche, alle opinioni sul mondo africano di coloniale memoria, che troppo spesso vengono considerati solo un ricordo del razzismo biologico ma che continuano, nella realtà, a lavorare anche nelle forme odierne di discriminazione (Tabet, 1997; Vaccarelli, 2008). Se omettiamo quelle aree geografiche rappresentate soltanto in casi isolati (Nord America, Europa mediterranea non UE, nello specifico Cipro, e il dato riferito ad un soggetto che non indica la provenienza) il livello di significatività del Chi Quadro risulta 0,035, il che conferma la tendenza individuata.

Anche rispetto all'orientamento religioso, possiamo rilevare alcune differenze. L'analisi è stata condotta non considerando quei casi (buddismo, induismo, bantu) per i quali si è ottenuta soltanto una risposta. Sono stati analizzate le risposte dei soggetti di religione cristiana e musulmana. Si precisa che i 24 soggetti che si sono dichiarati atei o agnostici si sentono o non si sentono colpiti da pregiudizi in maniera sostanzialmente uniforme (11 no, 13 sì). Nella Tabella 3 notiamo una lieve tendenza a concentrare i casi di pregiudizio sui soggetti di religione musulmana (con una differenza percentuale pari all'11,8\%). Il test esatto di Fisher, utilizzato nelle variabili dicotomiche, rileva tuttavia una significatività pari a 1,74 , che non ci permette di generalizzare il risultato.

Tab. 3 - Pregiudizi/Discriminazioni per orientamento religioso

\begin{tabular}{lccrcrc}
\hline & \multicolumn{2}{c}{ Si } & \multicolumn{2}{c}{ No } & \multicolumn{2}{c}{ Totale } \\
\hline & v.a. & $\%$ & v.a. & $\%$ & v.a. & $\%$ \\
\hline Musulmani & 49 & $52,7 \%$ & 44 & $47,3 \%$ & 93 & $100,0 \%$ \\
\hline Cristiani & 20 & $64,5 \%$ & 11 & $35,5 \%$ & 31 & $100,0 \%$ \\
\hline Totale & 55 & $44,4 \%$ & 69 & $55,6 \%$ & 124 & $100,0 \%$ \\
\hline
\end{tabular}

Si è cercato inoltre di verificare quanto l'esperienza del pregiudizio possa influire o condizionare altri ambiti dell'esperienza accademica e della percezione della vita e delle relazioni sociali in Italia (Tab. 4). Attraverso l'analisi delle medie condizionate e della varianza (Anova) abbiamo cercato di veri- 
ficare quanto questa esperienza ricada sui risultati accademici (misurati attraverso i crediti acquisiti e le votazioni medie), sulla valutazione della qualità dei servizi offerti dall'università, sugli atteggiamenti nei confronti degli italiani e degli stranieri, sull'indice di gradimento della cultura italiana, sulla motivazione (misurata a partire dal numero giornaliero di ore di studio). Si è inoltre verificata, con l'intento di sondare l'esistenza di una matrice classista nel definire i comportamenti discriminatori, l'associazione con l'indice di status sociale. Per quanto possiamo rilevare a partire dalle medie condizionate maggiori criticità nel gruppo che riferisce di essere stato esposto a pregiudizi/discriminazioni (ad esclusione del numero di CFU acquisiti), l'analisi della varianza segnala l'esistenza di differenze significative in tutti gli altri casi.

Tab. 4 - La presenza del pregiudizio sugli indicatori di rendimento e sugli atteggiamenti (medie condizionate e Anova)

\begin{tabular}{lrrc}
\hline Ti sei mai sentito colpito da forme di pregiudizio nell'ambiente accademico? \\
\hline Medie di: & Si & No & $\begin{array}{c}\text { Anova } \\
\text { significativo }\end{array}$ \\
\hline Indice di status (scala 0-10) & 5,6 & 5,8 & Lieve \\
\hline Numero di CFU acquisiti & 101,1 & 98 & NO \\
\hline Media voti & 23,7 & 24,6 & Sì \\
\hline Ore di studio al giorno & 7,5 & 6,9 & Sì \\
\hline Indice Qualità (scala 0-10) & 3,9 & 4,9 & Sì \\
\hline Indice Atteggiamento vs colleghi italiani & 5,4 & 6,4 & Sì \\
\hline Indice atteggiamento vs colleghi stranieri & 6,3 & 6,9 & Sì \\
\hline Indice cultura italiana & 5,2 & 5,5 & Sì \\
\hline
\end{tabular}

\subsection{Pregiudizi, socialità, atteggiamenti nei confronti de- gli italiani}

Nell'incrociare i dati relativi al pregiudizio con quelli riferiti alla socialità in ambito accademico (numero e nazionalità di amici e conoscenti), non sono state riscontrate differenze di distribuzione particolarmente significative. L'esperienza del pregiudizio, di per sé, non spiegherebbe necessariamente l'isolamento o una socialità ridotta. Tuttavia, se mettiamo in rapporto le due variabili con una terza, l'indice sintetico sugli atteggiamenti nei confronti degli italiani, possiamo individuare alcune interessanti tendenze statisticamente significative. Come osserviamo nella Tabella 5, al crescere del numero degli amici, il punteggio nell'indice sintetico riferito agli atteggiamenti nei con- 
fronti degli italiani si alza notevolmente, passando da 31,3 punti dei soggetti isolati ai 45,6 punti dei soggetti con più di 5 amici (a fronte di un punteggio medio complessivo di 39,6 punti). Anche internamente ai gruppi, se escludiamo la singolarità del sottogruppo che risponde di non avere amici (nel quale troviamo una leggera variazione in positivo tra i soggetti che hanno risposto di essere stati esposti a pregiudizi), notiamo che coloro che non riferiscono situazioni di discriminazione, manifestano atteggiamenti più favorevoli nei confronti degli italiani. L'analisi della varianza indica in questo caso una forte significatività statistica, pari a 0,002. Lo stesso trend, con significatività statistica soddisfacente ( $\mathrm{F}$ di Fisher con sig. 0,028), è rilevabile nel caso in cui si chiede il numero di "buoni conoscenti" (anche in questo caso, per motivi di sintesi, non vengono forniti dati analitici).

\section{Tab. 5 - Pregiudizio/Socialità/Atteggiamenti}

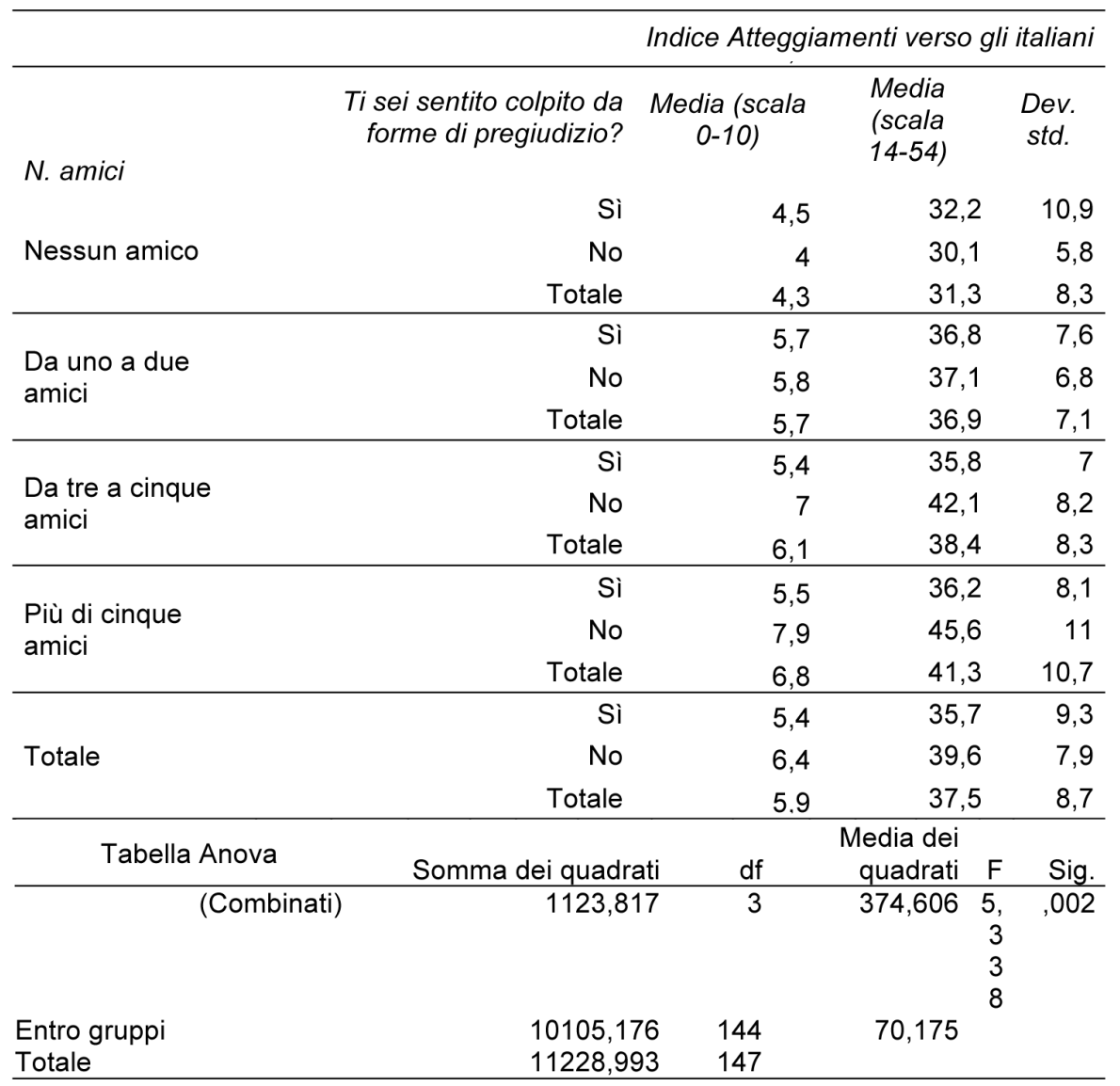




\subsection{Oltre il pregiudizio: il bisogno di mediazione e le possibili azioni}

Se i risultati della ricerca segnalano la presenza di pregiudizi nei confronti degli studenti di cittadinanza non italiana, possiamo individuare alcuni ambiti entro cui attivare politiche e interventi utili a prevenire o a contrastare quei pregiudizi che di fatto ostacolano gli studenti in quei processi di integrazione sociale che si traducono nella più ampia idea di qualità della vita e nella qualità dell'esperienza di vita e studio all'università. Possiamo assumere come concetti guida, quelli di advocacy ed empowerment, che già Fiorucci (2000) ha utilizzato per spiegare e orientare le pratiche della mediazione interculturale. Sul fronte dell'advocacy, è possibile ipotizzare azioni specifiche che costituiscano un riferimento chiaro, ad uso tanto dei singoli quanto dell'istituzione, in tema di diritti e della loro tutela. Sul fronte dell'empowerment, se consideriamo questo concetto in via generale (e dunque come potenziamento e sviluppo dell'autonomia, tanto degli individui quanto del sistema), le azioni potrebbero rimandare soprattutto allo sviluppo di un clima organizzativo e didattico propriamente riferito alla prospettiva internazionale (e interculturale) verso cui le politiche formative europee sembrano convergere. Per fare alcuni esempi riferiti contemporaneamente alle due dimensioni appena accennate, possono essere ipotizzate le seguenti azioni:

- elaborare un documento istituzionale che funzioni da parametro per gli attori interni dell'istituzione e da riferimento per gli stessi studenti in tema di diritti e della loro tutela) in cui siano enunciati non solo principi generali, ma anche regole da rispettare. L'azione dovrebbe previamente rivisitare trasversalmente le regolamentazioni dell'ateneo (Codice etico, Carta dei diritti dello studente in primis) e coinvolgere quelle figure di riferimento (garante degli studenti, referente di ateneo per l'internazionalizzazione, referenti nei vari dipartimenti, rappresentanze studentesche, ecc.), rispetto alle questioni inerenti i comportamenti da evitare o da mettere in atto.

- Rafforzare le competenze linguistiche (corsi di italiano di livello avanzato e corsi di italiano scientifico da erogare a inizio di ogni anno accademico ad esempio) e costruire protocolli di comportamento nella didattica e nella valutazione (al primo anno di iscrizione, privilegiare i test a scelta multipla, prevedere l'utilizzo della lingua inglese, nonché dei dizionari bilingui, ecc.).

- Mettere a regime e modellizzare buone pratiche di mediazione attraverso iniziative rivolte agli studenti, ma anche al personale docente e tecnico-amministrativo (formazione interculturale, a più livelli intesa). Su questo punto, che ci sembra particolarmente significativo, non ci soffermeremo, rinviando all'ultima parte del volume. 


\title{
14. Tempi e spazi per lo studio e per la vita sociale
}

\author{
di Marta Allevi, Francesca Palma
}

\subsection{Il contesto socio-territoriale della città dell'Aquila}

«Alcune connotazioni del reale possono essere colte solo assumendo il punto di vista spaziale, il quale consente di accedere contemporaneamente all'informazione referenziale (topografia e topologia) e ai significati simbolici (senso e valore) dei luoghi» (Calandra, 2012, p. 28).

Ed è questo il punto di vista che si vuole assumere in questo capitolo, uno sguardo che permetta di evidenziare come alcuni fondamenti del vivere insieme si definiscano a partire dallo spazio, considerato come una dimensione della realtà (Calandra, 2012). Lo spazio, scrive Franco La Cecla serve a distinguersi e a distinguere: «La mappa mentale di un insediamento è un'esperienza intersoggettiva» (La Cecla 1993, p. 33), costituita dall'incontro di corpi in movimento e da dinamiche sociali che si sviluppano intorno all'uso degli spazi pubblici della città e anche per gli studenti dell'università. Lo spazio e l'architettura possono essere considerati un elemento di controllo e pertanto uno strumento politico (Foucault, 2001). L'ordine socio-spaziale si riflette nel modo in cui gli individui si comportano e nel modo in cui organizzano le proprie pratiche quotidiane (Bourdieu, 2001). Lo spazio è un incrocio di entità in movimento, è «l'effetto prodotto dalle operazioni che l'orientano, lo circostanziano, lo temporalizzano e lo fanno funzionare come unità polivalente di programmi conflittuali o di prossimità contrattuali» (De Certeau, 2001, p. 176). Le pratiche sociali prendono luogo nello spazio e le pratiche quotidiane rappresentano uno strumento attraverso il quale le persone si riappropriano dello spazio che li circonda (Ibidem). In tal senso anche le semplici attività quotidiane sono parte dei processi di appropriazione, territorializzazione e producono

1. I paragrafi 14.1. e 14.4. vanno attribuiti a Francesca Palma, mentre i paragrafi 14.2. e 14.3. a Marta Allevi. 
un sentimento di appartenenza. La dimensione spazio-temporale che la città assume agli occhi di chi vi arriva, può ridurre o aumentare la "distanza" dal luogo di provenienza. Indagare lo spazio può di conseguenza aiutarci a capire il senso dello sradicamento di chi lascia la propria casa, la nostalgia dovuta alla perdita di abilità di orientamento nelle proprie mappe spaziali e la frustrazione di fronte alle difficoltà di radicamento su un nuovo territorio. La città dell'Aquila permette di accorciare la distanza tra il "qui" e il luogo d'origine degli studenti che vi arrivano per studiare? Una città le cui ferite, provocate dal sisma del 2009, sono ancora evidenti e il cui centro, un tempo catalizzatore in quanto sede di uffici, attività commerciali, mercato quotidiano, teatri, cinema, locali notturni, è oggi praticabile solo per una piccolissima parte e perlopiù votato alla vita notturna. «Le parole chiave della letteratura sulla globalizzazione, coincidono con gli elementi che emergono dal nuovo assetto urbano della città dell'Aquila, come si evince dalla voce di chi il nuovo assetto lo vive. Frammentazione, disgregazione, individualismo... caratterizzano la società aquilana del dopo sisma. Con la frammentazione dello spazio urbano si assiste anche al restringersi e alla scomparsa degli spazi pubblici (...)» (Palma, 2012, p. 121). In riferimento ad un contesto così complesso, andremo ad indagare come si muovono nello spazio gli studenti, da dove viaggiano, quali sono gli spazi pubblici che frequentano, dove sono collocati i dipartimenti, la percezione che hanno della città e dell'università, quanto si sentono coinvolti nelle problematiche della ricostruzione e la posizione degli studenti stranieri e internazionali rispetto al Paese d'origine.

\subsection{I flussi degli studenti}

Dalla ricerca emerge che degli studenti italiani intervistati il 55,8\% vive all'Aquila, il 38,2\% viaggia, il 6\% indica "altro". Una tendenza analoga la riscontriamo tra gli studenti stranieri scolarizzati in Italia: il 59,1\% di essi vive all'Aquila e il 40,9\% risulta pendolare. Tra gli studenti internazionali invece la percentuale dei domiciliati aumenta notevolmente ed è pari al $65,3 \%$, mente il $34,7 \%$ dichiara di essere pendolare. Nel Grafico 1 sono riportate le percentuali degli studenti, per i tre gruppi considerati, che viaggiano per frequentare l'università e che vivono all'Aquila. Tra gli studenti che vivono all'Aquila sono gli internazionali che perlopiù condividono un appartamento con altri colleghi (50\%), mentre gli studenti stranieri risultano essere in percentuale maggiore tra quelli che vivono in una residenza universitaria (7,3\%) o che vivono all'Aquila con la famiglia (24,2\%). Ricordiamo che infatti gli studenti internazionali tendono ad avere una situazione socioeconomica leggermente migliore rispetto agli altri (cfr. cap. 8). 


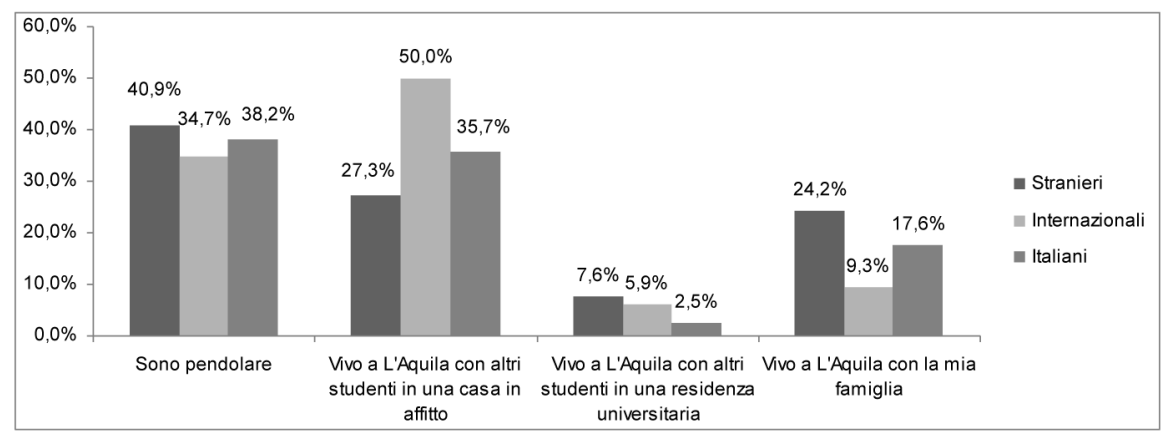

Considerata la notevole percentuale di chi si dichiara "pendolare" si è pensato dunque di entrare più nel dettaglio e analizzare da un punto di vista socio-territoriale questa fascia di studenti che scelgono la città dell'Aquila per il proprio percorso di studi, ma che di fatto usufruiscono in maniera diversa di tempi e luoghi rispetto ai loro colleghi che scelgono di trasferirsi nel capoluogo abruzzese.

Interessante, da un punto di vista geografico-spaziale, è l'analisi sulle provenienze che prende in considerazione il luogo di residenza degli studenti. Così come emerge dalle risposte fornite, si tratta in sostanza, di un pendolarismo perlopiù regionale che caratterizza tutti e tre i gruppi di studenti presi in considerazione (Tab. 1). Andando più nello specifico dell'analisi dei dati, si può sottolineare che le province maggiormente coinvolte in questo fenomeno di pendolarismo sono quelle limitrofe alla provincia aquilana e che fanno parte della regione Abruzzo: sommando i dati delle residenze si nota, infatti che dalla provincia dell'Aquila confluiscono il $37 \%$ dei pendolari, di cui il $26,7 \%$ sono studenti internazionali e gli studenti italiani sono oltre il $28 \%$. Le città della provincia aquilana maggiormente coinvolte da questo flusso sono Avezzano e Sulmona. Dalla provincia di Teramo provengono l'11\% degli studenti stranieri, quasi il 22\% degli studenti internazionali e, infine, il 16,5\% degli studenti italiani. In quest'ultimo caso l'ateneo aquilano, per chi risiede a Teramo, risulta facilmente accessibile poiché si tratta di un viaggio abbastanza breve e le due città sono discretamente collegate con mezzi pubblici e autostrada. Dalla provincia di Chieti e di Pescara provengono invece, esclusivamente di studenti italiani (3,1\% da Chieti e il $7 \%$ da Pescara); è ragionevole pensare che più degli italiani gli stranieri scolarizzati in Italia tendono a frequentare le università più vicine a loro, come in questo caso l'Università di Chieti-Pescara. 
Si può sottolineare anche che un flusso consistente riguarda la provincia di Rieti e quella di Roma e alcune zone del frusinate. Anche in questo caso, raggruppando i dati delle tre categorie selezionate per province si può notare che la provenienza di studenti dalla provincia di Rieti è notevole, quasi il 20\% e altrettanto dalla provincia di Roma: oltre il 54\% della somma totale dei pendolari arriva dalle zone del Lazio. Per quanto riguarda le altre province italiane, si tratta di percentuali relativamente basse che non influiscono molto sull'andamento degli iscritti totali.

Tab. 1 - Province di provenienza per studenti stranieri, internazionali, italiani

\begin{tabular}{|c|c|c|c|}
\hline & $\begin{array}{l}\text { Studenti } \\
\text { stranieri }\end{array}$ & $\begin{array}{c}\text { Studenti } \\
\text { internazionali }\end{array}$ & $\begin{array}{c}\text { Studenti } \\
\text { italiani }\end{array}$ \\
\hline & \multicolumn{3}{|c|}{ Provenienze dalle province abruzzesi } \\
\hline Prov. dell'Aquila & $37 \%$ & $26,7 \%$ & $28,6 \%$ \\
\hline Prov. di Teramo & $11,1 \%$ & $21,8 \%$ & $16, \%$ \\
\hline Prov. di Chieti & - & - & $3,2 \%$ \\
\hline \multirow[t]{2}{*}{ Prov. di Pescara } & - & - & $7,6 \%$ \\
\hline & \multicolumn{3}{|c|}{ Provenienze dalle altre province italiane } \\
\hline $\begin{array}{l}\text { Prov. di Ascoli } \\
\text { Piceno/Fermo }\end{array}$ & - & - & $1,2 \%$ \\
\hline Prov. di Avellino & - & - & $0,6 \%$ \\
\hline Prov. di Bologna & $3,7 \%$ & - & - \\
\hline Prov. di Foggia & - & - & $0,6 \%$ \\
\hline Prov. di Foligno & - & - & $1,8 \%$ \\
\hline Prov. di Formia & - & - & $0,6 \%$ \\
\hline Prov. di Frosinone & - & $2,4 \%$ & $1,8 \%$ \\
\hline Prov. di Latina & - & - & $0,6 \%$ \\
\hline Prov. di Lecce & - & - & $0,6 \%$ \\
\hline Prov. di Milano & - & $2,4 \%$ & - \\
\hline Prov. di Napoli & - & - & $0,6 \%$ \\
\hline Prov. di Perugia & - & - & $1,3 \%$ \\
\hline Prov. di Ravenna & - & - & $0,6 \%$ \\
\hline Prov. di Rieti & $11,1 \%$ & $4,9 \%$ & $5,1 \%$ \\
\hline Prov. di Roma & $22,5 \%$ & $19,4 \%$ & $12,7 \%$ \\
\hline Prov. di Terni & - & - & $0,6 \%$ \\
\hline Prov. di Trento & - & - & $0,6 \%$ \\
\hline Prov. di Verona & - & - & $0,6 \%$ \\
\hline Non risponde & $14,8 \%$ & $21,8 \%$ & $10,8 \%$ \\
\hline
\end{tabular}




\subsection{L'agire territoriale degli studenti: dove si muovono in città}

Come mostra la Figura 1, oggi la localizzazione dell'ateneo si distribuisce principalmente nella località di Coppito, frazione nord-ovest dell'Aquila a $5 \mathrm{~km}$ dal centro, luogo in cui trovano spazio i dipartimenti che fanno riferimento all'area scientifica. Si tratta, tra l'altro di strutture che hanno subito danni meno tangibili dopo l'evento sismico, attrezzando un campus tenda nei mesi successivi l'accadimento. Le strutture che ospitavano l'area ingegneristica localizzate, invece, storicamente a sud-ovest nella frazione di Roio, hanno trovato sede nel polo industriale e commerciale di Pile. Il polo umanistico ha subito più danni dal punto di vista del patrimonio immobiliare dell'ateneo: ad esempio, la storica sede in centro della ex Facoltà di Lettere e Filosofia, infatti, era stata ri-localizzata nell'area periferica di Bazzano, per poi trovare finalmente spazio nei pressi del centro storico nel 2012 costituendo, insieme alla ex Facoltà di Scienze della Formazione, il Dipartimento di Scienze Umane.

Vale la pena riconsiderare la distribuzione degli studenti per dipartimento, già presa in esame nel capitolo 9 , in ragione della loro collocazione nello spazio urbano. L'ateneo aquilano ha da sempre attirato un gran numero di studenti internazionali per quanto riguarda le discipline scientifiche e, in particolare quello che oggi è il Dipartimento di Medicina clinica, sanità pubblica, scienze della vita e dell'ambiente (MESVA), è quello che attira la gran parte degli studenti italiani e di altra cittadinanza in totale; un'alta

Fig. 1 - L'ateneo aquilano, 2015: localizzazione dei dipartimenti (elaborazione dati google maps engine)

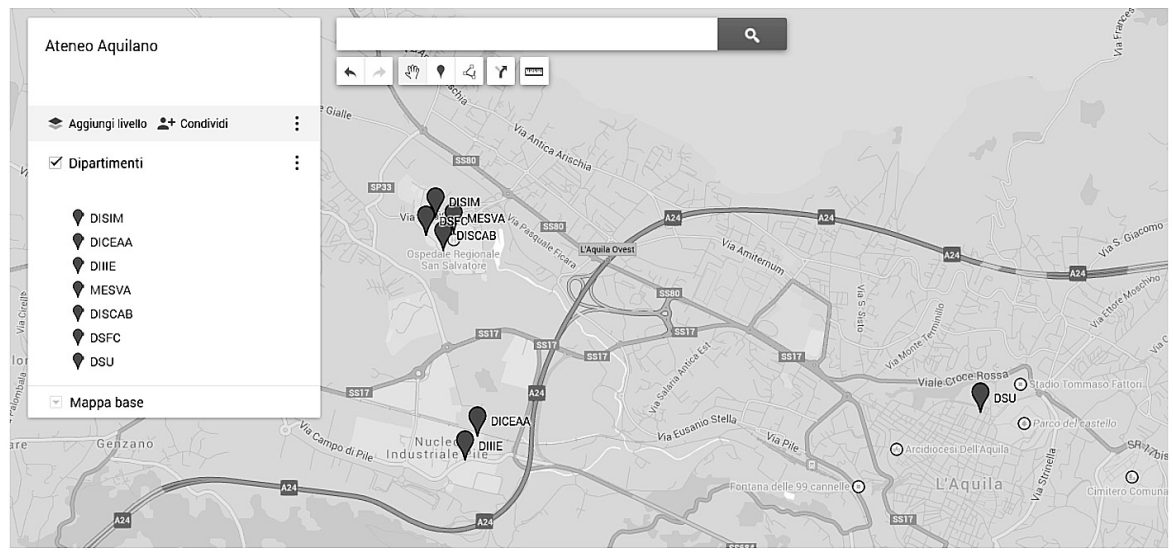


percentuale degli studenti rispondenti al questionario, invece, fa riferimento al Dipartimento di Ingegneria industriale e dell'informazione e di economia (DIIIE), dove gli stranieri complessivamente si attestano sul 15\% e gli italiani oltre il $18 \%$. Un altro dipartimento che rileva un discreto numero di iscritti è quello di Scienze Umane (DSU).

Da un punto di vista geografico, mettere in relazione i luoghi utilizzati e frequentati dagli studenti mette in evidenza anche la qualità degli stessi e l'adeguatezza o meno degli spazi utilizzati dalla comunità stessa. Che tipo di spazi offre oggi la città dell'Aquila ai suoi studenti? Da che punto di vista, qualitativo o quantitativo?

A sei anni dal sisma del 2009, per gli aquilani il centro è luogo di memorie e di ricordi, di una vita passata più rassicurante e meno caotica e dispersiva, sforzandosi di "inventare" un altro modo di vivere lo spazio urbano per adeguarsi in fretta alle nuove condizioni abitative e territoriali del post-sisma (Allevi, 2012; Calandra, 2012). Tuttavia, anche per la comunità di studenti che vive la sua quotidianità nella città si richiede uno sforzo enorme di adeguamento alle condizioni che la città ha imposto. Il centro storico, quello che era il cuore pulsante della città, ha di fatto modificato la sua funzione di catalizzatore sociale e culturale non solo per gli aquilani, ma anche per gli studenti che perlopiù vivevano tutto ciò che il centro storico aveva loro da offrire: servizi, vita universitaria, ma anche divertimento. Ora, se tutto ciò che riguarda la vita universitaria si è spostato "fuori", il centro storico si trova ad offrire solo vita notturna e locali, pub e birrerie, delegando tutto ciò che ha a che fare più in generale con la vita sociale ad altri spazi. A quali spazi, però, possiamo fare riferimento? In che modo questi vengono utilizzati e percepiti?

I dati raccolti permettono di dare conto di una componente importante del territorio e del vissuto quotidiano di una comunità di studenti che intesse relazioni, si muove, si incontra in uno spazio che dovrebbe permettere loro di vivere appieno il periodo degli studi.

E dunque, come vivono gli studenti all'Aquila, oggi? Dove agiscono territorialmente questi soggetti e quali sono le pratiche sociali che li caratterizzano? Come si configura l'agire territoriale degli studenti all'Aquila in seguito allo stravolgimento urbano - ma non solo - che ha coinvolto l'intera comunità? Da questo punto di vista, infatti, è molto interessante approfondire il dato in riferimento agli spazi e ai luoghi frequentati dagli studenti attraverso l'analisi della domanda "Di solito dove vi incontrate" che scatta una fotografia unica sul rapporto studente/territorio e spazio percepito/spazio vissuto (Tab. 2). 
La Tabella 2 mostra i luoghi di incontro degli studenti, intesi come spazi in cui trascorrere tempo e dedicare i momenti della socialità. A questo si aggiunge, oltre che uno spazio fisico e geografico, anche uno spazio "virtuale" in cui trascorrere il tempo libero, ovvero i social network: i dati raccolti mettono in evidenza come in termini attuali si dedica molto tempo della quotidianità all'utilizzo dei social media, strumenti privilegiati nel mantenere contatti, intessere relazioni, coltivare amicizie.

Tab. 2 - I luoghi di incontro degli studenti

\begin{tabular}{lccc}
\hline & $\begin{array}{c}\text { Studenti } \\
\text { stranieri }\end{array}$ & $\begin{array}{c}\text { Studenti } \\
\text { internazionali }\end{array}$ & $\begin{array}{c}\text { Studenti } \\
\text { italiani }\end{array}$ \\
\hline A casa & $31,8 \%$ & $40,7 \%$ & $32,2 \%$ \\
\hline Al bar/locali/pub & $28,8 \%$ & $22,9 \%$ & $30,4 \%$ \\
\hline All'università & $81,8 \%$ & $72,9 \%$ & $82,4 \%$ \\
\hline Centri/associazioni sportive & $3 \%$ & $9,3 \%$ & $3,5 \%$ \\
\hline Centri/associazioni culturali & $4,5 \%$ & $5,9 \%$ & $4,3 \%$ \\
\hline Centri religiosi/Luoghi di culto & - & $3,4 \%$ & $1,5 \%$ \\
\hline In giro per la città & $27,3 \%$ & $28,8 \%$ & $23,9 \%$ \\
\hline Negozi/centri commerciali & $12,1 \%$ & $16,9 \%$ & $6,5 \%$ \\
\hline Social network: Facebook, & $48,5 \%$ & $47,5 \%$ & $33,7 \%$ \\
Whatsapp, Skype, ecc. & & & \\
\hline
\end{tabular}

Tuttavia, se volgiamo lo sguardo sull'agire territoriale della comunità studentesca, a livello locale gli spazi di incontro sono diversificati: tra tutti, spicca l'università come luogo privilegiato di ritrovo, in riferimento agli spazi messi a disposizione dell'ateneo e nelle sedi dei dipartimenti (biblioteche, aule studio, corridoi...); poi la voce che raccoglie un maggior numero di scelte è "a casa", decretando forse un tipo di socialità più "intima" e privata, tra amici a dispetto di un'esigenza di viversi la città nei suoi spazi pubblici, il centro storico con le sue piazze, i vicoli, i portici. Se gli studenti aquilani vanno poco "in giro per la città" e preferiscono le abitazioni private, dove altro trascorrono il loro tempo in città dopo le ore dedicate allo studio? Le voci che riguardano "centri/associazioni sportive", "centri/associazioni culturali" e "centri religiosi/luoghi di culto" dimostrano percentuali molto basse; i negozi e i centri commerciali ricoprono un ruolo discreto, tuttavia sono i "bar/locali/pub" i luoghi in cui gli studenti tendono a trascorrere la maggior parte del tempo libero. Nello specifico, inoltre, si tratta di locali che si collocano nelle zone percorribili e vivibili del centro storico, ma anche in zone della città - prima percepite come periferiche e marginali - 
che negli ultimi anni hanno trovato una differente funzionalità. Ad esempio, strade come il "Viale della Croce Rossa" che in epoca pre-sisma svolgeva da collegamento tra la parte del centro storico e la zona ovest della città, già dal 2010 vi trovarono sede numerosi locali, pub, birrerie creando non pochi problemi - specialmente nelle ore notturne e di massimo afflusso della clientela - alla circolazione e alla sicurezza, in quanto lo spazio era probabilmente inadatto per il traffico e un flusso così alto di pedoni. Negli anni questa tendenza a creare zone dedicate alla "movida notturna" non è cambiata e molti locali del centro si trovano costretti a chiudere, per lasciare spazio all'avviata ricostruzione degli edifici e a ricollocarsi in altri ambienti. In sostanza, dai dati raccolti emerge che la socialità studentesca è vissuta principalmente tra le mura degli edifici universitari e, presumibilmente nelle ore notturne, ha un rapporto solo con alcuni luoghi della città e una scarsa conoscenza del complesso tessuto urbano aquilano. La tendenza, così come riportano i dati, dà conto di una forma del vissuto quotidiano e dello spazio un po' stravolta che propende verso una tipologia di abitare atopico, la quale racchiude in sé una forma di rifiuto del luogo che si lega a sentimenti di temporaneità, provvisorietà, insicurezza (Allevi, 2013).

Infatti, nella domanda è stato chiesto di specificare più approfonditamente "per le opzioni che hai selezionato nella domanda precedente, indica quali sono i luoghi che frequentate (es: nome del locale, associazioni, ecc.)" e ciò che ne viene fuori è una mappa della socialità che mette in rilievo esclusivamente locali notturni e bar della città. In pochi indicano genericamente "il centro storico", pochissimi dichiarano Piazza Duomo o Piazza Regina Margherita come luogo scelto per la socialità quotidiana; la gran parte dei rispondenti, dunque, frequenta abitualmente pub e birrerie come spazi dedicati allo svago e non include nelle risposte un tipo di socialità "pubblica".

A ciò l'università ha voluto rispondere con iniziative, interne ed esterne al progetto, che vedono coinvolti i tavoli partecipativi i quali muovono una dinamica diversa dell'uso della città e dello spazio.

\subsection{L'esperienza degli studenti: la città e l'università}

Come percepiscono la qualità della vita all'Aquila gli studenti? Che valutazione danno agli spazi per lo studio e per la vita sociale? Andiamo a vedere come percepiscono e valutano la loro esperienza di vita sociale, urbana, ecc., in città e in ambito accademico. In questo senso abbiamo fatto riferimento alle scale di "valutazione della vita sociale all'Aquila", 16 indicatori riguardanti le condizioni abitative, il tempo libero, l'organizzazione del territorio, ecc. Un approfondimento del dato - distinguendo tra studenti 
internazionali e studenti stranieri scolarizzati in Italia - lo abbiamo ottenuto attraverso le medie riferite ad ognuno degli indicatori (i punteggi sono riportati su scala 0-10) (Tab. 3). Abbiamo ritenuto utile altresì distinguere, tra gli studenti che vivono all'Aquila e gli studenti che viaggiano per frequentare l'università (Tab. 4).

Tab. 3 - Percezione e valutazione della qualità della vita sociale e urbana nei gruppi di studenti

\begin{tabular}{lcccc}
\hline & $\begin{array}{c}\text { Studenti } \\
\text { stranieri }\end{array}$ & $\begin{array}{c}\text { Studenti } \\
\text { internazionali }\end{array}$ & $\begin{array}{c}\text { Studenti } \\
\text { italiani }\end{array}$ & $\begin{array}{c}\text { Anova con F } \\
\text { significativa }\end{array}$ \\
\hline Condizioni abitative generali & 3,35 & 2,53 & 3,34 & No \\
\hline Prospettive occupazionali & 2,55 & 2,28 & 2,45 & No \\
\hline Servizi sociosanitari & 4,43 & 4,67 & 4,13 & No \\
\hline $\begin{array}{l}\text { Relazioni interpersonali e vita } \\
\text { sociale }\end{array}$ & 4,69 & 3,96 & 4,24 & No \\
\hline $\begin{array}{l}\text { Servizi per il pubblico offerti } \\
\text { da uffici e istituzioni }\end{array}$ & 3,87 & 3,71 & 3,20 & No \\
\hline Intrattenimento e svago & 3,00 & 3,10 & 3,23 & No \\
\hline Stimoli culturali & 3,47 & 3,32 & 3,33 & No \\
\hline $\begin{array}{l}\text { Spazi per il tempo libero e } \\
\text { luoghi di aggregazione }\end{array}$ & 3,00 & 2,95 & 3,05 & No \\
\hline $\begin{array}{l}\text { Trasporti e mobilità sul } \\
\text { territorio urbano }\end{array}$ & 3,21 & 2,74 & 2,47 & No \\
\hline Assetto urbano & 2,87 & 2,85 & 1,93 & Sì \\
\hline Sicurezza & 4,45 & 5,89 & 3,66 & Sì \\
\hline Qualità del paesaggio & 6,50 & 5,97 & 6,25 & No \\
\hline Qualità del territorio & 5,55 & 5,56 & 5,57 & No \\
\hline Vita politica & 2,41 & 3,06 & 2,75 & No \\
\hline Luoghi di culto e spiritualità & 3,84 & 4,62 & 4,53 & No \\
\hline \begin{tabular}{l} 
Indice Qualità della vita \\
\hline
\end{tabular} & 4,05 & 3,98 & 4,30 & No \\
\hline
\end{tabular}

Le maggiori criticità si riscontrano per gli indicatori riferiti a: assetto urbano, prospettive occupazionali, condizioni abitative generali, vita politica, spazi per il tempo libero e luoghi di aggregazione, trasporti e mobilità sul territorio urbano, servizi per il pubblico offerti da uffici e istituzioni. Risultano, infatti, punteggi medi molto inferiori a 5, limite minimo di accettabilità. Gli indici: stimoli culturali, servizi socio-sanitari, relazioni interpersonali e vita sociale, luoghi di culto e spiritualità e qualità della vita riportano medie un po' più alte ma sempre al di sotto del minimo. Lieve- 
Tab. 4 - Percezione e valutazione della qualità della vita sociale e urbana: domiciliati e pendolari

\begin{tabular}{lcccc}
\hline & Domiciliato & Pendolare & Totale & \\
\hline & Media & Media & Media & $\begin{array}{c}\text { Anova con F } \\
\text { significativa }\end{array}$ \\
\hline Condizioni abitative generali & 3,12 & 3,29 & 3,20 & No \\
\hline Prospettive occupazionali & 2,30 & 2,61 & 2,43 & No \\
\hline Servizi socio sanitari & 4,23 & 4,34 & 4,25 & No \\
\hline $\begin{array}{l}\text { Relazioni interpersonali e } \\
\text { vita sociale }\end{array}$ & 4,03 & 4,51 & 4,24 & No \\
\hline $\begin{array}{l}\text { Servizi per il pubblico offerti } \\
\text { da uffici e istituzioni }\end{array}$ & 3,02 & 3,88 & 3,36 & Sì \\
\hline Intrattenimento e svago & 2,92 & 3,59 & 3,19 & Sì \\
\hline Stimoli culturali & 3,01 & 3,81 & 3,34 & Sì \\
\hline $\begin{array}{l}\text { Spazi per il tempo libero e } \\
\text { luoghi di aggregazione }\end{array}$ & 2,70 & 3,55 & 3,03 & Sì \\
\hline $\begin{array}{l}\text { Trasporti e mobilità sul } \\
\text { territorio urbano }\end{array}$ & 2,30 & 3,04 & 2,60 & No \\
\hline Assetto urbano & 1,95 & 2,51 & 2,18 & No \\
\hline Sicurezza & 4,22 & 3,95 & 4,12 & No \\
\hline Qualità del paesaggio & 6,49 & 5,92 & 6,23 & No \\
\hline \begin{tabular}{l} 
Qualità del territorio \\
\hline Vita politica
\end{tabular} & 5,82 & 5,24 & 5,56 & No \\
\hline Luoghi di culto e spiritualità & 4,40 & 4,63 & 4,48 & No \\
\hline Indice di Qualità della vita & 4,12 & 4,34 & 4,22 & No \\
\hline
\end{tabular}

mente superiori ai limiti minimi di accettabilità abbiamo i punteggi medi riferiti alla qualità del territorio e alla qualità del paesaggio. Non emergono in generale differenze sostanziali, rispetto ai tre gruppi, tranne da due indici: l'assetto urbano che gli italiani valutano in maniera più negativa; la sicurezza, per la quale osserviamo che la media riferita agli studenti internazionali supera largamente il minimo di accettabilità, mentre per gli altri due gruppi le medie risultano al di sotto del minimo (la media dei punteggi assegnati dagli italiani è la più bassa). Per entrambi gli indici abbiamo Anova con F significativo, fatto che ci permette di considerare tali differenze come altamente significative. Condizionando i punteggi del campione totale rispetto al domicilio degli studenti, otteniamo risultati che ci permettono di distinguere le percezioni di chi vive in città e di chi inve- 
ce la frequenta da pendolare e verificare se uno dei due gruppi condizioni le medie totali. Emerge una valutazione generale, leggermente più positiva da parte dei pendolari. Differenze che possiamo considerare altamente significative, Anova con F significativa, riguardano gli indici: servizi per il pubblico offerti da uffici e istituzioni, intrattenimento e svago, stimoli culturali, spazi per il tempo libero e luoghi di aggregazione che i pendolari percepiscono in maniera più positiva con medie che restano comunque al di sotto del minimo di accettabilità.

Le valutazioni negative che gli studenti esprimono rispetto alla città potrebbero essere legate al nuovo assetto dell'Aquila post-sisma. Qual è il livello di coinvolgimento degli studenti nei problemi relativi alla ricostruzione della città? Dalle risposte alla domanda "Nella tua esperienza di studio all'Aquila, quanto ti senti coinvolto nelle vicende legate ai problemi della ricostruzione?", emerge che il coinvolgimento degli studenti italiani è maggiore, la media dei punteggi supera abbondantemente il minimo accettabile 5, le medie degli altri due gruppi non lo raggiungono. Tra gli studenti stranieri sono maggiormente coinvolti gli studenti che vivono in città (Tab. 5).

Tab. 5 - Coinvolgimento nelle vicende legate ai problemi della ricostruzione postsisma nei tre gruppi di studenti

\begin{tabular}{lcccc}
\hline & $\begin{array}{c}\text { Studenti } \\
\text { stranieri }\end{array}$ & $\begin{array}{c}\text { Studenti } \\
\text { internazionali }\end{array}$ & $\begin{array}{c}\text { Studenti } \\
\text { italiani }\end{array}$ & $\begin{array}{c}\text { Anova con } \\
\text { F significativo }\end{array}$ \\
\hline Totale & 4,06 & 3,64 & 6,85 & Sì \\
\hline Domiciliati & 4,43 & 4,02 & 6,78 & Sì \\
\hline Pendolari & 3,45 & 2,95 & 6,94 & Sì \\
\hline
\end{tabular}

Tutti gli intervistati denunciano la mancanza all'Aquila di luoghi dedicati alla cultura del proprio Paese (ristoranti, alimentari, circoli e associazioni, luoghi di culto, luoghi di incontro, ecc.). Dichiarano che le cose di cui sentono maggiormente la mancanza e che qui non trovano sono innanzitutto il cibo e a seguire la famiglia e il calore umano. Gli studenti stranie$r i$ sono quelli che dichiarano, in percentuale più alta $(53,8 \%)$, di sentire la mancanza del proprio Paese ma di voler costruire il proprio futuro altrove. Gli studenti internazionali che sentono molto la mancanza vogliono anche tornarci a vivere $(28,6 \%)$. Il 23,1\% degli studenti stranieri dichiara di non sentirne affatto la mancanza (Graf. 2). 


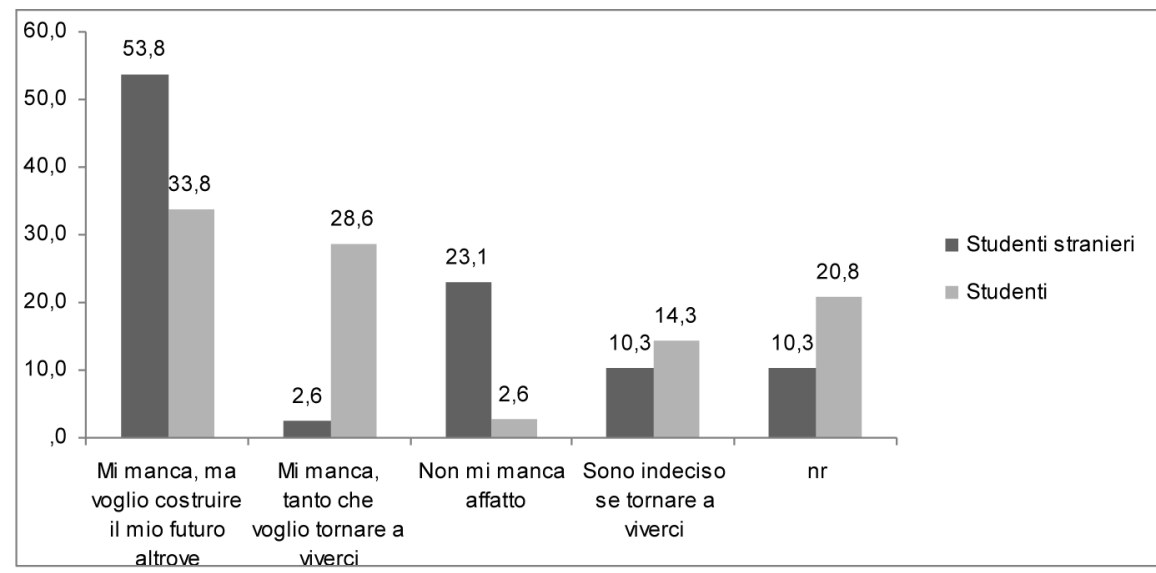

Altro aspetto preso in considerazione è la "valutazione della qualità dell'università", riferito ad 8 indicatori: tutorato, servizi di segreteria, sistemi di diffusione delle informazioni, qualità della didattica, strutture di supporto allo studio, diritto allo studio, logistica, chiarezza della modulistica e delle procedure amministrative (Tab. 6). Come si può osservare nella Tabella 2, l'unico indicatore che supera il minimo accettabile si riferisce alla qualità della didattica per tutti i gruppi. Gli studenti internazionali danno una valutazione leggermente più positiva degli altri due gruppi. Differenze che possiamo considerare altamente significative, Anova con F significativa, riguardano gli indici: servizi di segreteria, per gli studenti internazionali quasi sufficiente, la media è di poco sotto il minimo, per gli altri due gruppi i punteggi sono molto più bassi; strutture di supporto allo studio, in questo caso la media dei punteggi assegnati dagli studenti stranieri è uguale al minimo accettabile mentre le medie degli altri gruppi sono sotto, con il punteggio più basso assegnato dagli italiani; per il diritto allo studio e la logistica sono gli italiani a dare una valutazione più negativa.

Il quadro che emerge, dagli indicatori presi in esame, è quello di una città e di conseguenza di una università, che soffrono ancora i danni provocati dal sisma. L'università ricopre tuttavia un ruolo importante, infatti, gli spazi messi a disposizione dell'ateneo nelle sedi dei dipartimenti (biblioteche, aule studio, corridoi...) costituiscono il luogo di ritrovo privilegiato degli studenti, che li reputano però insufficienti come si evince dalla Tabella 6. La media dei punteggi assegnati alla logistica non raggiunge il minimo accettabile; altri spazi pubblici frequentati sono quasi esclusivamente "bar/locali/pub". Si denuncia, anche rispetto alla città, la mancanza 
di spazi per il tempo libero e luoghi di aggregazione il cui indice riporta un punteggio medio molto inferiore al minimo, come si può vedere nella Tabella 3. I dati offrono uno spaccato della vita all'Aquila a 6 anni dal terremoto non molto diverso da quello rappresentato da ricerche precedenti: "Qualità della vita e motivazione allo studio degli studenti dell'Università dell'Aquila. Emergenza e post-emergenza" (Isidori e Vaccarelli, 2012); L'"Iniziativa C.As.A, Comunicazione per l'Ascolto Attivo"' (Calandra, 2012).

Tab. 6 - La valutazione dell'università

\begin{tabular}{lcccc}
\hline & $\begin{array}{c}\text { Studenti } \\
\text { stranieri }\end{array}$ & $\begin{array}{c}\text { Studenti } \\
\text { internazionali }\end{array}$ & $\begin{array}{c}\text { Studenti } \\
\text { italiani }\end{array}$ & \\
\hline Media & Media & Media & $\begin{array}{c}\text { Anova con } \\
\text { F significativo }\end{array}$ \\
\hline Tutorato & 3,33 & 3,61 & 2,90 & No \\
\hline Servizi di segreteria & 3,92 & 4,85 & 3,72 & Sì \\
\hline $\begin{array}{l}\text { Sistemi di diffusione } \\
\text { delle informazioni }\end{array}$ & 4,63 & 4,82 & 4,21 & No \\
\hline Qualità della didattica & 5,88 & 5,59 & 5,73 & No \\
\hline $\begin{array}{l}\text { Strutture di supporto } \\
\text { allo studio }\end{array}$ & 5,00 & 4,46 & 3,81 & Sì \\
\hline \begin{tabular}{l} 
Diritto allo studio \\
\hline Logistica
\end{tabular} & 3,58 & 4,01 & 2,94 & Sì \\
\hline $\begin{array}{l}\text { Chiarezza della modulistica } \\
\text { e delle procedure } \\
\text { amministrative }\end{array}$ & 3,63 & 4,25 & 3,48 & Sì \\
\hline
\end{tabular}


Parte III

Le azioni positive e lo sguardo al futuro 



\title{
15. La progettazione partecipata: il gruppo come strumento per promuovere la convivenza
}

\author{
di Maria Marronaro, Farnaz Mirzapoor, Jean Pierre Ndayambaje ${ }^{1}$
}

\subsection{Introduzione}

Nel progetto "Unidiversità" i tavoli di partecipazione sono stati istituiti per avviare e facilitare il processo di progettazione partecipata in materia di politiche migratorie, con l'obiettivo di programmare azioni ed interventi a favore di cittadini stranieri residenti nel territorio aquilano.

Per "progettazione partecipata", definizione che si rifà al vocabolo inglese partnership, utilizzato dalle scienze politico-sociali di scuola anglosassone, si intende una modalità di collaborazione tra $\mathrm{i}$ vari attori sociali al fine di perseguire un obiettivo sociale e, indirettamente, un vantaggio per i partecipanti ad un progetto. Il concetto partnership è connesso ad un'idea di concertazione, più dinamica e focalizzata sui bisogni della comunità e sull'assunzione di responsabilità dei diversi attori anche in un ottica di sussidiarietà, welfare mix e responsabilità.

Lo strumento delle partnership si basa sulla convinzione che le tematiche legate allo sviluppo socio-economico e culturale di un territorio non siano materia di soli governi e amministrazioni, ma prima ancora della comunità e della società civile, e che, nel processo di programmazione, attuazione e controllo dello stesso, governi e amministrazioni devono avere il ruolo di facilitazione e di animazione di accordi di collaborazione sul territorio (Ascoli, 2003). Nel progetto "Unidiversità", il ruolo dell'Università degli Studi dell'Aquila e delle associazioni è stato pertanto quello di attivare, rafforzare e diffondere nelle istituzioni locali e nell'intera società aquilana questa cultura dell'agire insieme nei processi di cambiamento socio-culturale attualmente in atto.

1. Jean Pierre Ndayambaje è autore del paragrafo 15.1.; a Maria Marronaro vanno attribuiti i paragrafi 15.2., 15.3. e 15.4.; a Farnaz Mirzapoor i paragrafi 15.5. e 15.6. 
Costruite sulla lunga tradizione europea degli accordi sociali, le partnership rappresentano un'importante fonte di innovazione, sia per le azioni e le pratiche locali che per le politiche di interventi nel campo socio-culturale più in generale. Per essere innovativa una politica locale che si basa sulle partnership deve:

- coinvolgere nuovi attori oltre alle tradizionali rappresentanze;

- condividere le priorità di intervento con la comunità attraverso opportune forme di dialogo e coinvolgimento.

Le azioni fin qui messe in atto da varie istituzioni locali sia pubbliche che private al fine di coinvolgere gli stakeholders hanno messo in luce un intento meramente formale, che non incide significativamente sul cambiamento culturale, e quindi duraturo, che tutte le politiche orientate alla partecipazione devono presupporre. I limiti più evidenti si rilevano nella superficialità e discontinuità di questi interventi, nella mancata valorizzazione delle competenze e delle expertise informali dei cittadini e delle reti naturali presenti nelle comunità locali.

In questo progetto si è partiti dal presupposto che la partecipazione alla progettazione, alla realizzazione e alla valutazione dei servizi e delle politiche migratorie, si può costruire solo a partire dalla connessione con le risorse formali e istituzionali, delle esperienze di vita e delle naturali capacità di coping che i cittadini e le comunità mettono in atto affrontando le diverse evenienze della quotidianità. Risulta perciò indispensabile dotarsi di prospettive di lettura adatte alla comprensione delle diverse realtà, delle singole esperienze di vita, della peculiarità delle azioni di problem solving che coinvolgono gli individui, le famiglie, le comunità, le varie organizzazioni associative presenti sul territorio, trasformando questo enorme capitale sociale in risorsa collettiva.

La politica proposta nel progetto mette in evidenza quello che mina le scelte di società e si proietta verso il futuro per realizzare la seguente visione: una società aquilana inclusiva e fiera della sua diversità, che aspira ad una maggiore coesione sociale attraverso la partecipazione di tutti alla vita collettiva e che vede l'immigrazione come una risorsa essenziale per il suo sviluppo.

Questo è l'intento con cui è nato il Coordinamento RicostruireInsieme subito dopo il sisma del 6 aprile 2009 per cercare di canalizzare gli interventi messi in atto dal mondo delle associazioni a favore degli immigrati. Le nostre azioni sono state principalmente realizzate nei campi di accoglienza dove la convivenza forzata ha, in un certo senso, riportato in superficie le differenze e le diversità socio-culturali sfociando, anche spesso, in situazioni di conflitto aperto. In queste condizioni, il ruolo del no- 
stro Coordinamento è stato quello di ammortizzatore con azioni di prevenzione e/o di mediazione nei conflitti. In seguito alla chiusura dei campi, la maggior parte della popolazione aquilana è stata sistemata negli alloggi provvisori delle new town disseminati sul territorio aquilano creando nuovi problemi legati alla dislocazione e alla disgregazione del tessuto sociale già profondamente percosso dal sisma. Attualmente, il Coordinamento RicostruireInsieme in collaborazione con altri attori sociali (associazioni, organismi e istituzioni pubbliche e private), è impegnato nella fase della cosiddetta "ricostruzione sociale" ragion per cui è partner del progetto "Unidiversità".

È grazie alla partecipazione di tutta la popolazione, in tutte le sue diversità e nel rispetto dei principi fondamentali della società moderna, che i legami di solidarietà che uniscono gli aquilani e le aquilane si rafforzeranno e si tradurranno in una maggiore coesione sociale. Il progetto ha adottato un approccio globale che si articola su grandi temi rispetto ai quali le società moderne fanno fronte per realizzare la visione proposta, che è quella di una società inclusiva e fiera della sua diversità. Per ogni sfida, alcune scelte strategiche sono prese in considerazione al fine di individuare le azioni in grado di fornire una risposta adeguata alle preoccupazioni sollevate. Un primo tema ricorda il principio di impegno condiviso in cui l'integrazione, la partecipazione e l'inclusione coinvolgono, allo stesso tempo, sia persone che sono impegnate attivamente nella edificazione di una "nuova società aquilana" sia la società, nel suo complesso, che riconosce e apprezza il contributo di tutti. Essendo una città plurale, L'Aquila deve essere inclusiva. Quindi, si deve garantire che nessuno sia lasciato fuori sulla base delle differenze etno-culturali reali o percepite, consentendo la piena partecipazione di tutte le estrazioni socio-culturali ed economiche in conformità alle linee guida imposte dalla cultura pubblica comune. Le società che non si preoccupano di promuovere l'inserimento, favoriscono le dinamiche di emarginazione e di ripiegamento culturale, terreno fertile per le tensioni sociali. Un altro tema è una chiamata all'azione di coinvolgimento che si avvale del contributo e della collaborazione di tutte le parti interessate in un approccio integrato. Essa mira tra le altre cose a sviluppare un quadro di gestione che offra una leva strutturale tale da permettere il raggiungimento, con pertinenza, efficacia ed efficienza, degli obiettivi della politica. Una politica che non scommette su uno sforzo globale concertato, rischia di trovarsi nell'impossibilità di andare oltre l'intenzione del discorso e tradursi in modo efficace nella realtà.

Nell'intento di dare seguito a ciò, nella seconda fase del progetto "Unidiversità", sono stati dislocati nelle varie sedi dei dipartimenti dell'ateneo aquilano e nei luoghi di aggregazione degli studenti universitari (come ad esempio la casa dello studente "Campomizzi") quattro tavoli di partecipa- 
zione, per far sì che questi ultimi potessero dare il loro contributo individuando azioni, eventi ed attività da realizzare nell'ultima fase del progetto. Nella stessa occasione, si è offerta ai partecipanti l'opportunità di indicare proposte di intervento ed azioni da includere come priorità nei futuri progetti elaborati sia dalle amministrazioni pubbliche locali sia da quelle private, sia, ancora, dal mondo delle organizzazioni ed associazioni che operano nel territorio aquilano sui temi dell'immigrazione. Il quinto ed ultimo tavolo, si è tenuto presso il Coordinamento RicostruireInsieme, quale partner del progetto; in tale sede sono stati invitati a partecipare i rappresentanti delle associazioni di immigrati e gli immigrati operanti nel territorio aquilano, che hanno sempre denunciato e criticato il modo di procedere nell'azione di programmazione degli interventi a beneficio degli immigrati da parte delle istituzioni pubbliche (comuni, province e regioni) senza il loro coinvolgimento. È da sottolineare che il coinvolgimento non deve limitarsi alla sola fase progettuale ma anche a quelle dedicate alla implementazione, alla valutazione e al monitoraggio degli obiettivi raggiunti al fine di un'eventuale azione di ridefinizione degli stessi. Per questo motivo, ci auspichiamo che l'azione dei tavoli, sopra descritti, prosegua anche dopo la conclusione del progetto realizzato.

\subsection{La formazione del gruppo dei tutori della convivenza}

Nelle azioni progettuali, il coinvolgimento della comunità è stato affidato a venti persone che sono state definite tutori della convivenza, opportunamente selezionate attraverso un bando pubblico, che hanno rappresentato una leva fondamentale nell'implementazione degli obiettivi e delle attività previste. Prima di rivolgersi all'esterno, i tutori hanno affrontato un periodo di formazione con una serie di incontri seminariali e laboratoriali riguardanti i temi dell'immigrazione sotto diversi punti di vista: dalla descrizione del fenomeno in termini statistici e demografici, alla presentazione delle leggi che regolano i flussi migratori in Italia, ai fenomeni di discriminazione razzista.

La fase successiva ha riguardato la formazione dei tutori da un altro punto di vista, esperenziale e riflessivo: si è pensato che, prima di arrivare ai tavoli di progettazione, fosse fondamentale lavorare sia sul piano individuale sia sul rafforzamento del senso di appartenenza al gruppo. L'obiettivo a lungo termine del percorso che ci siamo prefissati è stato quello di costruire un gruppo dove ci fosse oltre che la condivisione di un lavoro comune dal punto di vista teorico e pratico, anche uno scambio emotivo e relazionale. La motivazione e l'impegno, infatti, vengono sviluppati attraverso delle buone relazioni di lavoro ed un senso di appartenenza al grup- 
po (Rosa, 1999). Inoltre l'idea è stata quella di costruire un gruppo dove il senso di appartenenza coincidesse con la definizione di una matrice comune che permettesse di condividere scopi e idee e che successivamente potesse declinarsi nella creazione di diversi tavoli di progettazione; in essi il ruolo dei tutori sarebbe poi stato quello di coinvolgere altre persone, restituendo loro i presupposti teorici, la motivazione e gli obiettivi che si sono sviluppati nel contesto del gruppo allargato.

Nel lavoro con i tutori abbiamo cercato di costruire insieme questo senso di appartenenza, tenendo sempre presenti le dinamiche gruppali che inevitabilmente si sviluppano quando diverse persone entrano in relazione per un periodo duraturo (Neri, 2003). In psicologia sociale il gruppo è inteso come un insieme di individui che interagiscono tra loro influenzandosi reciprocamente e che condividono, più o meno consapevolmente, interessi, scopi, caratteristiche e norme comportamentali (Galimberti, 1999). È quindi evidente l'importanza dell'esistenza di un legame perché si possa parlare di gruppo. Lewin (1972) aggiunge a questo concetto l'idea che il gruppo è un sistema autonomo che non può essere conosciuto e gestito solo a partire dalla conoscenza dei singoli elementi che lo compongono, perché questi si influenzano vicendevolmente in modo sistematico, rendendo ciascun gruppo diverso dall'altro e diverso dalla semplice somma algebrica delle singole parti. Quando, come formatrici, ci siamo avvicinate al gruppo dei tutori eravamo consapevoli di tale specifica caratteristica e abbiamo cercato di essere elementi catalizzatori di questa. Inoltre abbiamo tentato di passare questo concetto anche ai tutori stessi tenendo presente un principio che ha guidato i nostri interventi e che è quello di un isomorfismo tra il gruppo di formazione dei tutori e i tavoli di progettazione che si sarebbero creati successivamente. Ciò che accadeva nel gruppo dei tutori, rispetto alle dinamiche personali e gruppali si sarebbe potuto ripetere nel gruppo di progettazione così che imparare a riconoscere e gestire tali dinamiche per i tutori avrebbe significato anche poter riconoscere e gestire in prima persona ciò che accadeva nei gruppi da loro condotti.

\subsection{Il gruppo come risorsa}

Il gruppo può essere considerato sia una modalità organizzativa che un "luogo" di comunicazione nel quale si verificano specifiche dinamiche relazionali. Un aspetto di cui abbiamo tenuto ampio conto è la differenza tra gruppo di lavoro e lavoro di gruppo. Il primo è un'unità organizzativa che opera per raggiungere un obiettivo comune in un dato periodo di tempo; il secondo è una modalità di lavoro, un metodo che permette di conseguire 
l'obiettivo prefissato coordinando e facilitando la relazione tra persone diverse tra loro per ruoli, competenze e obiettivi personali (Rosa, 1999). In ogni gruppo di lavoro sono presenti due aree, quella dei compiti e quella delle relazioni, e vi si succedono, anche se non in modo consequenziale, diverse fasi di vita: orientamento, conflitto, integrazione, interdipendenza. Questa ultima fase è quella più difficile da raggiungere ma è anche quella in cui il gruppo raggiunge il suo più alto livello di empowerment e in cui i membri si sentono maggiormente potenti, poiché si percepiscono parte di un tutto, necessari e dipendenti l'uno dall'altro per il raggiungimento degli obiettivi comuni. Si raggiungono pertanto due obiettivi importanti, contemplati anche nelle premesse teoriche del progetto "Unidiversità", dove all'aumento dell'empowerment del gruppo è associato il potenziamento di quello individuale, in un gioco di rinforzo reciproco che permette di portare cambiamenti importanti nei due sistemi interagenti. Nella fase dell'interdipendenza le diverse attività sono svolte proficuamente con collaborazione e partecipazione. È importante per raggiungere l'obiettivo dell'interdipendenza che le persone vengano formate a lavorare in gruppo, visto che questa è una competenza apprendibile, in modo che comprendano i benefici del lavoro di gruppo e il valore di una comunicazione efficace e di relazioni costruttive ed empowering nel contesto in cui si trovano ad interagire.

Per riconoscere e sviluppare queste competenze si è proposta al gruppo un'attività, nella quale si è chiesto di progettare una "macchina" che rendesse più facile la vita lavorativa dell'impiegato che si trova a contatto con le questioni burocratiche riguardanti gli immigrati, con lo scopo di dare ai partecipanti un'opportunità di innovazione, creatività e divertimento mentre lavorano in gruppi che concorrono l'uno con l'altro. Scopo dell'attività è stato far riflettere $\mathrm{i}$ tutori su come $\mathrm{i}$ gruppi possano lavorare produttivamente e contro-produttivamente e quale possa essere il loro contributo individuale. Nel prendervi parte, i tutori hanno potuto mettere in pratica le loro capacità e fare un uso complementare dei loro talenti: alcuni sono stati capaci di dirigere la discussione del gruppo, altri sono stati abili con le parole e sono riusciti a trovare un buon nome per la "macchina", alcuni si sono comportati come dei bravi diplomatici che hanno posto fine ai conflitti nell'ambito del gruppo, altri sono stati buoni strateghi che richiamavano l'attenzione del gruppo sul compito principale. L'esercitazione ha previsto delle domande inerenti il processo del gruppo ponendo l'attenzione su ciò che permette al gruppo di lavorare efficacemente o alternativamente su che cosa ostacoli il processo. Questo ha costretto alcuni individui ad ascoltare delle critiche riguardo a come essi abbiano lavorato nel loro gruppo e a decidere se considerarle valide, invece di mettersi subito sulla difensiva o sentirsi arrabbiati. 


\subsection{Gruppo ed emozioni nella formazione dei tutori della convivenza}

I tutori sono stati stimolati ad una riflessione interna sul modo di leggere la realtà che li circonda con l'obiettivo di promuovere una maggiore consapevolezza dei propri vissuti e delle proprie emozioni. In questo modo si è cercato di rompere alcuni degli schemi precostituiti con cui ci si rapporta all'altro, facendo vivere a ciascun tutore l'esperienza di riconoscere nel proprio modo di avvicinarsi al mondo la presenza di categorie rigide di valutazione che testimoniano l'esistenza di pregiudizi anche all'interno di se stessi, quale modalità più "economica" di conoscere ciò che li circonda.

L'intento è stato raggiunto attraverso l'utilizzo di tecniche "rompighiaccio". Il termine "rompighiaccio" evoca le navi speciali che portano questo nome, che vengono usate per rompere il ghiaccio nelle regioni artiche. E proprio nello stesso modo in cui queste navi facilitano il passaggio di altre navi, un "rompighiaccio" serve ad aprire la strada verso l'apprendimento consentendo ai partecipanti al gruppo di conversare in un ambiente più rilassato ed incoraggiante. In modo più specifico, un "rompighiaccio" è un'attività pensata per aiutare le persone a conoscersi tra loro, di solito tramite presentazioni e scambio di nomi e altre informazioni personali. Noi le abbiamo proposte nella parte iniziale di ciascun incontro con l'intento di perturbare le aspettative dei partecipanti e di trasmettere un meta-messaggio riguardante il modo di leggere la realtà. L'effetto, atteso e poi rilevato, scaturito dal vivere un'esperienza originale, divertente, a volte imbarazzante, è stato effettivamente quello di ridurre i vissuti persecutori che si sviluppano nei contesti gruppali. Le tecniche usate consistevano nell'utilizzo di presentazioni fatte in una lingua inventata al momento oppure nel salutarsi con il semplice sguardo. Oltre agli scopi sopra esplicitati, abbiamo pensato che far vivere ai tutori una situazione imbarazzante e a volte frustrante, potesse aiutarli a rompere determinati schemi culturali che si portavano dietro oltre che aiutarli a mettersi nei panni dell'altro, per chi non avesse già vissuto sulla sua pelle questa esperienza, in previsione del loro contatto con le diverse "visioni del mondo" che avrebbero incontrato nei tavoli di progettazione. La rottura degli schemi rigidi che i tutori portavano con sé era rivolta sia a quelli italiani che a quelli stranieri nel tentativo di passare il messaggio che non esiste una cultura che è migliore dell'altra: i pregiudizi nei confronti dell'altro sono presenti sia nei soggetti appartenenti sia alla cultura predominante sia a quella minoritaria.

Nel corso degli incontri abbiamo tentato di trasmettere l'idea di una complessità della convivenza tra le diverse "culture" che non si limitasse alla semplice contrapposizione tra cultura occidentale e altre culture, tra 
buoni e cattivi, proponendo degli stimoli provenienti dalle cosiddette figure ambigue della psicologia della Gestalt. Stati di ambivalenza e instabilità nel campo percettivo producono quello che è conosciuto come il fenomeno delle figure ambigue. Questo tipo di figura è caratterizzato dal fatto che le diverse componenti del campo percettivo assumono ruoli diversi a seconda del significato globale che si assegna all'immagine (Canestrari e Godino, 1997). Famosa è l'immagine di Hill (Fig. 1) che può dare adito a due rappresentazioni diverse: il volto di un'anziana signora, visto di profilo, oppure il volto di una giovane donna di scorcio.

Fig. 1 - La giovane e la suocera di Hill (1915)

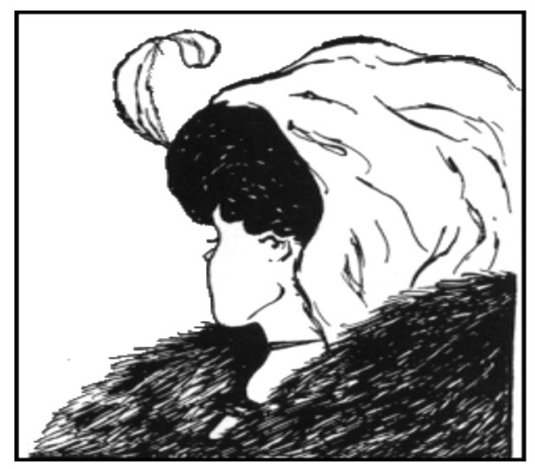

Nel gruppo, laddove qualcuno vedeva un'immagine, qualcun altro poteva coglierne una diversa, pur rimanendo gli elementi a disposizione dell'osservatore immutati. Questo ci ha permesso di porre l'accento su come la realtà può essere letta dagli osservatori in modi assai diversi pur rimanendo la stessa. Il nostro intento è stato trasmettere questa consapevolezza, al fine di aiutare i tutori ad avere gli strumenti per leggere il proprio conflitto interno e il conflitto tra altri soggetti che si sarebbe potuto porre davanti a loro nella gestione della convivenza tra le culture. Per questo il conflitto va gestito tenendo presente che nella lettura dei fenomeni sociali ognuna delle parti può dare versioni diverse a seconda del punto di vista che sceglie, ma che l'altra parte guarda la stessa realtà soltanto con occhi diversi. Utilizzando il principio dell'isomorfismo tra ciò che accadeva nel gruppo e ciò che sarebbe potuto accadere nei tavoli di progettazione e nella società più ampia abbiamo posto l'accento anche sui conflitti presenti nel gruppo stesso e sul modo di risolverli. Il dibattito e le riflessioni che ne sono scaturite sono state, a nostro avviso, un buon 
esercizio per i tutori per allenarsi a gestire i conflitti che hanno poi incontrato fuori del contesto protetto del gruppo di formazione. È passata l'idea che i conflitti sono inevitabili e per questo bisogna saperli riconoscere, imparare a gestirli e mediarli in chiave positiva. È importante vederli come un'espressione di diversità, un momento di crescita, sia personale che del gruppo, e come una possibilità di migliorare le relazioni piuttosto che come problema negativo. Una delle regole peculiari che abbiamo voluto sottolineare è che da un conflitto risolto non devono uscire né vinti né vincitori, ma persone soddisfatte di aver trovato un punto di incontro. Attuare delle strategie di mediazione del conflitto in una società dove gli scontri, i disguidi e le incomprensioni sono all'ordine del giorno, si rivela un'azione di basilare importanza. Si dà così valore a preservare i rapporti umani, senza negare le differenze, accettando i punti di vista degli altri. I sociologi americani Bush Baruch e Folger (2009) credono nel concetto di recognition (riconoscimento reciproco) come base fondante della mediazione: le parti che sono in conflitto non solo ricevono un supporto per risolvere il problema, ma vengono motivate affinché possano visionare e riconoscere consapevolmente possibili conflitti futuri. Baldry e Ardone (2003) asseriscono che l'apprendimento e l'applicazione di metodi per gestire il conflitto sono i mezzi più idonei per rendere il pensiero di trasformare un episodio conflittuale in un'occasione di scambio e di confronto, un atto concreto.

\subsection{Chi è il tutore della convivenza?}

Il lavoro con il gruppo è proseguito nella direzione di avviare un dibattito sul concetto stesso di tutore della convivenza, andando a sviscerare quelle che sono le rappresentazioni mentali che ciascun partecipante al gruppo ha di questa figura che dovrà mettere in atto nel contesto sociale. Il termine rappresentazione mentale è stato inteso nella sua accezione più generale, come un modo in cui viene elaborata l'esperienza, che si costituisce per rappresentare il mondo esterno e se stessi allo scopo specifico di un'interazione efficace dell'individuo con la realtà (Galimberti, 1999). Il termine così inteso e utilizzato è stato riferito al concetto di "immagine", al fine di avere una rappresentazione speculare di ciò che i tutori immaginavano di andare a mettere in scena nel contesto sociale. Il lavoro si è svolto utilizzando la tecnica del brainstorming per avere delle informazioni più generali sull'idea di tutore, che sono servite ad innescare una discussione in cui, oltre a condividere e spiegare il proprio punto di vista, si è andati costruendo una definizione condivisa del concetto di tutore della convivenza. Scopo di questa attività è stato quello di produrre una maggiore consapevolezza 
sul ruolo svolto da ciascuno, oltre che raggiungere un concetto condiviso da tutto il gruppo che rendesse più agevole la fase di implementazione del lavoro sul campo che i tutori sarebbero andati a svolgere. Ci preme riportare in questa trattazione lo svolgimento del processo che ha portato ad una definizione condivisa. Durante il brainstorming abbiamo chiesto ai tutori di porsi a turno come facilitatori, dando loro la responsabilità di condurre il gruppo. Contemporaneamente le formatrici riportavano le loro definizioni su cartoncini colorati che venivano appesi su uno spago che attraversava l'intera aula. Tutrici e tutori, dopo aver formulato svariate definizioni di tutore della convivenza, hanno riflettuto e lavorato attorno alle molte "parole"e "concetti" espressi da ciascuno di loro e si sono sforzati di semplificare, scegliere, fondere insieme quelli che sembravano loro più appropriati a definire il ruolo e le principali qualità del tutore della convivenza. Il metodo di conduzione di questo lavoro è stato quello dell'ascolto attivo delle opinioni del gruppo, su cui a turno, ciascuno di loro, si è esercitato a svolgere una funzione di facilitatore. Non è stato facile selezionare tra le idee, ma è stata sicuramente un'occasione di riflessione e analisi, un esercizio attivo alla scelta condivisa. Il dibattito si è acceso particolarmente nell'analizzare alcune interpretazioni proposte, come quella del tutore inteso come mediatore culturale, educatore culturale, o perfino come Batman o arbitro culturale. Dalla riflessione del gruppo è scaturito che nessuna di queste fosse la definizione più efficace per individuare il compito principale del tutore della convivenza. Al contrario, questo è stato il passaggio più importante per riflettere sulla neutralità che il tutore dovrebbe conservare, ferma restando una grande capacità empatica che deve comunque contraddistinguerlo. Inizialmente è stato quasi scontato intenderlo come un risolutore di problemi o di conflitti di natura soprattutto "culturale", ma man mano che la riflessione si è approfondita, è sembrato opportuno fare emergere sin dalla definizione stessa un'accezione positiva del suo ruolo, mettendo quindi l'accento sul concetto di soluzione, anziché su quello di conflitto. Sono quindi arrivati a concordare che il tutore non è colui che risolve problemi, piuttosto è colui il quale fa emergere delle soluzioni. Si è ragionato a lungo anche sull'opportunità di definire con l'aggettivo "culturale" la natura delle soluzioni che il tutore è chiamato a proporre, ma a ben riflettere, e forse memori di alcuni stimoli forniti durante il corso di formazione sul concetto di "cultura", "transcultura" e "intercultura", si è focalizzato il fatto che i problemi cui fa fronte il tutore della convivenza non hanno origine nelle culture in sé, ma nella convivenza tra culture. Tra le definizioni proposte in partenza, c'era quella di attivatore di processi, che è sembrata non discostarsi troppo dalla definizione "ideale". Alla responsabilità di attivare processi si è preferito focalizzare l'attenzione sul concetto di gestione; alla generica definizione di processi si è deciso di specificare il genere di 
processi "conflittuali" che il tutore si trova a gestire: facilitatore di processi di convivenza è sembrata quindi la definizione più corretta da cui partire. Un secondo momento di analisi e cernita ha riguardato l'individuazione delle principali qualità personali ed attitudinali del tutore della conviven$z a$. Un'intensa riflessione si è sviluppata attorno ad alcune qualità personali che erano state proposte inizialmente, in particolare quelle di sforzarsi ad essere aconfessionale e apolitico: pur apparendo secondo alcuni delle qualità scontate e in un certo senso implicite, il gruppo ha deciso di ribadire questi attributi, a garanzia di un lavoro che deve essere condotto senza pregiudizi di alcuna origine o natura. Al contrario è sembrato improprio fare salva la qualità personale di democracità o diplomaticità, preferendo ad esse quella più attitudinale di essere garante dell'equilibrio tra le parti. Questa definizione è stata scelta al fine di esprimere meglio o sostituire anche altri concetti proposti, come quello di garante dei diritti o facilitatore di apprendimento reciproco. Ne è risultato che, secondo il gruppo, una definizione di tutore della convivenza può essere quella di facilitatore dei processi di convivenza, che ha il compito di promuovere l'intercultura e facilitare il dialogo, rivalutare le diversità diffondendone il valore attraverso la promozione di iniziative e di idee di pace e convivenza: una figura capace di catalizzare la crescita personale e della collettività intera. Ci è sembrato importante riportare questa ampia descrizione del dibattito sulla rappresentazione del tutore della convivenza perché è stato un modo per co-costruire con il gruppo e con i nostri contributi di conduttori un paradigma per un lavoro sul campo.

\subsection{I tavoli di progettazione partecipata}

I tavoli di progettazione partecipata previsti dal progetto "Unidiversità" si sono andati costituendo attraverso il coinvolgimento di studenti italiani e di altra cittadinanza e di associazioni che operano sul territorio attraverso il lavoro delle tutrici e dei tutori. Primo passo per il gruppo dei tutori è stato suddividersi nei cinque sottogruppi come proposto dal progetto e individuare delle strategie per coinvolgere altri soggetti e avviare i tavoli di progettazione. Si è pensato alla costruzione di un poster con un'immagine comune che gli studenti e le associazioni potessero riconoscere come punto di riferimento e una parte che riportasse i contenuti dei singoli tavoli di progettazione. Per promuovere la diffusione del progetto si è proposto l'utilizzo dei social network oltre che l'organizzazione di una conferenza stampa alla quale hanno partecipato la Rettrice e tutti i soggetti che hanno preso parte al progetto. I sottogruppi hanno lavorato facendo una valutazione iniziale rispetto a quelle che erano le possibili esigenze che avrebbe- 
ro potuto portare gli eventuali interlocutori. Come era indicato nel progetto sono sorti cinque gruppi o tavoli di progettazione: Creare diversa-mente, Unidiversità Città, Roio per il mondo, Parliamone a colori, Unidiversità Coppito. Creare diversa-mente è il gruppo che è nato presso la Casa dello studente, un contesto in cui convivono numerose culture e dove ci si è confrontati con i problemi legati al vivere quotidiano come la creazione di spazi comuni da condividere nel tempo libero. Sulla base di questa esigenza in questo gruppo si è pensato di proporre la presentazione delle storie di vita di ragazzi che hanno avuto il desiderio di farsi conoscere e far conoscere il loro Paese d'origine. Il gruppo Unidiversità Città ha incontrato i rappresentanti delle varie associazioni di immigrati presenti sul territorio, ma anche le associazioni che potessero essere interessate a costruire qualcosa. La principale criticità incontrata dai tutori appartenenti a questo gruppo è stata quella di riuscire a risvegliare l'entusiasmo e la volontà di un cambiamento nelle associazioni di immigrati presenti sul territorio già da anni, che avevano sperimentato delle azioni nel contesto aquilano senza ottenere risultati soddisfacenti. Gli altri tre gruppi sono stati realizzati nell'ambito del contesto dell'università: Parliamone a colori si è riunito presso il Dipartimento di Scienze Umane, Unidiversità Coppito presso il polo bio-medico di Medicina e Scienze, Roio per il mondo presso il polo di Ingegneria. In questi contesti sono state contattate le varie associazioni studentesche come elemento di raccordo con gli studenti stessi sia italiani che stranieri. La risposta, almeno inizialmente, è venuta soltanto da pochi studenti stranieri poiché la maggioranza dei componenti erano italiani. Un'idea interessante per coinvolgere gli studenti è venuta dal gruppo Roio per il mondo dove i tutori hanno pensato di mettere a confronto studenti italiani e stranieri per sapere come vivono la loro esperienza universitaria, ma soprattutto per scoprire il mondo lavorativo. È stata proposta un'immagine ribaltata dell'immigrato che giunge in Italia per chiedere lavoro in colui che può fornire opportunità di lavoro perché proveniente da un Paese che in questo momento della sua storia ricerca delle figure professionali di cui non dispone. Una proposta che ha incuriosito diversi studenti e che ha inserito i partecipanti in una cornice fortemente motivante. Un aspetto importante della fase di progettazione partecipata è stato vedere come la fase di formazione e di crescita personale dei tutori sia stata fondamentale per attivare il processo di coinvolgimento e di motivazione delle altre persone implicate. Il concetto dell'isomorfismo, sopra esposto, tra gruppo dei tutori e gruppi di progettazione si è realizzato ed ha permesso di raggiungere quegli importanti obiettivi di sviluppo dell'empowerment dell'individuo e delle associazioni che permettono a queste ultime e al cittadino di sentirsi parte attiva della società. 
Il concetto di empowerment e le strategie che favoriscono il suo sviluppo a livello individuale e sociale, che si pongono alla base del progetto "Unidiversità", possono offrire modalità significative per esplorare nuovi modi di integrare crescita personale e sostegno sociale (Francescato e Giusti, 1999). Il processo attraverso cui si diventa empowered prevede una presa di coscienza dei limiti e delle opportunità offerte dai contesti sociali, e un coinvolgimento attivo della persona nei suoi contesti ambientali per renderli più confacenti ai suoi desideri. In quest'ottica si lavora contemporaneamente per aumentare le opportunità ambientali e per responsabilizzare gli individui a trarne vantaggio. 

Allegati 



\title{
I questionari per gli studenti italiani e di altra cittadinanza
}

\author{
di Maria Grazia Ferretti, Alessandro Vaccarelli ${ }^{1}$
}

\section{Unione Europea - Ministero dell'Interno \\ Fondo Sociale per l'integrazione dei cittadini di Paesi Terzi \\ Università degli Studi dell'Aquila - RicostruireInsieme - Comitato Territoriale ARCI dell'Aquila}

L'Università dell'Aquila, in partenariato con le associazioni RicostruireInsieme e Comitato Territoriale ARCI dell'Aquila, e con il sostegno dell'associazione Bibliobus dell' Aquila, ha avviato le attività del progetto denominato "Unidiversità". Il progetto è finanziato dal Ministero dell'Interno nell'ambito del Fondo Europeo per l'Integrazione dei cittadini di Paesi terzi (FEI) - annualità 2013. Nel quadro di tale iniziativa, stiamo conducendo un'indagine conoscitiva sulle condizioni di vita e di studio degli studenti italiani e stranieri. La ricerca sarà orientata ad individuare i principali bisogni e le possibili azioni strategiche tese a facilitare l'inserimento e l'integrazione in ambito universitario e cittadino. Sulla base dei dati ottenuti, saranno progettate ulteriori azioni e in particolare saranno attivati, presso tutti i dipartimenti, alcuni tavoli di progettazione partecipata aperti agli studenti e alle studentesse dell'Aquila che, partendo dall' analisi dei bisogni espressi dalla comunità studentesca, elaboreranno delle risposte cercando di superare i conflitti imputabili alle differenze culturali, nel rispetto dei valori e degli stili di vita di ciascuno.

Facendo parte del campione che abbiamo individuato, ti chiediamo di rispondere in tutta sincerità alle domande. Naturalmente garantiamo il rispetto del più assoluto anonimato. Le risposte che fornirai verranno trattate statisticamente in forma aggregata, per cui sarà impossibile a chiunque poter risalire alla tua persona.

1. Gli allegati sono stati revisionati ed organizzati da Maria Grazia Ferretti, che ha curato anche la rappresentazione dei dati. I questionari sono stati ideati da Alessandro Vaccarelli. 


\section{QUESTIONARIO STUDENTI DI CITTADINANZA NON ITALIANA}

Garantiamo l'assoluto anonimato e la riservatezza delle informazioni che ci saranno fornite nel rispetto della normativa sulla privacy

\section{DATI GENERALI}

1. Sesso: $\square$ Maschio $43,2 \% \quad \square$ Femmina $56,8 \%$

2. Età:

3. Luogo di nascita:

4. Cittadinanza:

5. Religione: $\square$ Nessuna 14,6\% $\square$ Musulmana 20,5\% $\square$ Cristiana 62,7\%
$\square$ Ebraica
$\square$ Buddista $0,5 \%$
$\square$ Induista $1,6 \%$
$\square$ Altro

6. Sei un credente praticante? $\square$ Molto $15,8 \% \quad \square$ Abbastanza $31 \%$

$\square$ Poco $36,7 \% \quad \square$ Per niente $16,5 \%$

\section{Da quanto tempo sei in Italia?}

\begin{tabular}{lr}
\hline Meno di sei mesi & $1,1 \%$ \\
\hline Da sei mesi a dodici mesi & $4,9 \%$ \\
\hline Da uno a due anni & $7,0 \%$ \\
\hline Da due a cinque anni & $11,9 \%$ \\
\hline Da più di cinque anni & $74,6 \%$ \\
\hline Sono nato in Italia & $0,5 \%$ \\
\hline
\end{tabular}

\section{Comune di residenza:}

\section{Dove abiti:}

Vivo all'Aquila con altri studenti in una casa in affitto (indicare quartiere o frazione: ...) $42,2 \%$

Vivo all'Aquila con altri studenti in una residenza universitaria $6,5 \%$

Vivo all'Aquila con la mia famiglia $14,6 \%$

Sono pendolare $36,8 \%$

Altro: 


\section{Dove vive la tua famiglia?}

Nazione

Città

11. Titolo di studio dei genitori (indica quello corrispondente al titolo italiano)

\begin{tabular}{lcccccc}
\hline & Nessuno & $\begin{array}{c}\text { Scuola } \\
\text { elementare }\end{array}$ & $\begin{array}{c}\text { Scuola } \\
\text { media }\end{array}$ & $\begin{array}{c}\text { Qualifica } \\
\text { professionale }\end{array}$ & $\begin{array}{c}\text { Scuola } \\
\text { superiore }\end{array}$ & Laurea \\
\hline Madre & $3.2 \%$ & $5,4 \%$ & $16,2 \%$ & $14,1 \%$ & $30,3 \%$ & $30,8 \%$ \\
\hline Padre & $2,7 \%$ & $4,9 \%$ & $11,9 \%$ & $19,5 \%$ & $24,3 \%$ & $36,8 \%$ \\
\hline
\end{tabular}

12. Puoi indicare la fascia di reddito della tua famiglia in Italia/nel tuo Paese?

$\square$ Bassa $43,8 \%$

$\square$ Medio/bassa $29,7 \%$

$\square$ Media $22,2 \%$

Medio/alta 3,8\%

$\square$ Alta $0,5 \%$

\section{Chi paga i tuoi studi?}

$\square$ La mia famiglia $39,5 \%$

$\square$ Li pago da solo $25,9 \%$

$\square$ La mia famiglia mi aiuta, ma cerco di contribuire personalmente $24,3 \%$

$\square$ Ho una borsa di studio italiana $7 \%$

$\square$ Ho una borsa di studio internazionale 3,2\%

\section{Svolgi qualche attività lavorativa?}
$\square$ Sì, lavoro $18,9 \%$
Sì, lavoro ma saltuariamente $26,5 \%$

$\square$ No, studio soltanto $54,6 \%$

\section{Tipo di permesso di soggiorno:}

$\square$ Permesso per ricongiungimento familiare $9,7 \%$

$\square$ Permesso per motivi di studio 44,9\%

$\square$ Carta di soggiorno $26,5 \%$

$\square$ Asilo politico $0,5 \%$

$\square$ Permesso scaduto/in attesa di rinnovo 2,7\%

$\square$ Non ho il permesso di soggiorno $15,7 \%$ 


\section{LA SCUOLA}

\section{Scuola superiore frequentata}

$\square$ Liceo ad indirizzo umanistico e/o scientifico $65,7 \%$

$\square$ Istituto tecnico-Istituto Professionale 34,3\%

Specificare

17. Voto finale del diploma di scuola superiore (da 60 a 100/100)

\begin{tabular}{rccc}
\hline $60-70$ & $71-80$ & $81-90$ & $91-100$ \\
\hline $13,5 \%$ & $33,7 \%$ & $23 \%$ & $25,3 \%$ \\
\hline
\end{tabular}

Nel mio caso non è prevista una valutazione finale con giudizio o voto $4,5 \%$

\section{Puoi indicarci la tua condizione?}

$\square$ Sono nato in Italia e ho frequentato la scuola in Italia $1,7 \%$

$\square$ Sono arrivato in Italia con la mia famiglia (o raggiungendo la mia famiglia) e ho frequentato almeno in parte la scuola italiana $36,5 \%$

Sono arrivato in Italia per frequentare l'università $61,8 \%$

19. In base alle tue esperienze, la scuola italiana quanto è in grado di supportare i ragazzi stranieri nelle seguenti aree?

\begin{tabular}{lrrrr}
\hline & Molto & Abbastanza & Poco & Per niente \\
\hline Lingua italiana & $18,1 \%$ & $52,0 \%$ & $23,7 \%$ & $6,2 \%$ \\
\hline Lingue straniere & $5,6 \%$ & $29,2 \%$ & $46,6 \%$ & $18,5 \%$ \\
\hline $\begin{array}{l}\text { Apprendimenti nelle discipline } \\
\text { umanistiche }\end{array}$ & $16,8 \%$ & $53,2 \%$ & $22,5 \%$ & $7,5 \%$ \\
\hline $\begin{array}{l}\text { Apprendimenti nelle discipline } \\
\text { tecnico-scientifiche }\end{array}$ & $14,5 \%$ & $62,4 \%$ & $19,1 \%$ & $4,0 \%$ \\
\hline $\begin{array}{l}\text { Attività artistiche e/o sportive } \\
\text { Integrazione sociale e benessere }\end{array}$ & $11,0 \%$ & $43,0 \%$ & $30,2 \%$ & $15,7 \%$ \\
\hline \begin{tabular}{l} 
scolastico \\
\hline
\end{tabular} & $95,4 \%$ & $43,4 \%$ & $12,0 \%$ \\
\hline
\end{tabular}

\section{LA FORMAZIONE LINGUISTICA}

\section{Hai avuto difficoltà ad imparare la lingua italiana?}

$\square$ Molte 5,6\% $\square$ Abbastanza 24,7\% $\square$ Poche 39,9\% $\square$ Per niente 29,8\%

21. Qual è il tuo attuale livello di conoscenza della lingua italiana?

$\square$ Ottimo 52,8\% $\square$ Buono 36,5\% $\square$ Sufficiente 7,9\% $\square$ Scarso 2,8\% 
22. Conoscevi l'italiano prima di arrivare?
Molto bene 3,4\%
$\square$ Abbastanza bene $15,2 \%$
$\square$ Poco $27,5 \%$
$\square$ Per niente $53,9 \%$

\section{Hai frequentato dei corsi di lingua italiana? \\ $\square$ Sì $58,4 \%$ \\ $\square$ No $41,6 \%$}

\section{Se sì, dove?}

$\square$ Nella scuola italiana $20,4 \%$

$\square$ Corsi di lingua italiana organizzati nel mio Paese $44,7 \%$

$\square$ Corsi di lingua svolti in Italia 20,4\%

$\square$ Autoformazione $14,6 \%$

Altro (specificare)

25. Possiedi una certificazione linguistica di lingua italiana?
Sì $67,4 \%$
$\square$ No $32,6 \%$

26. Se sì, di che livello?
$\square$ A1 $18,2 \%$
$\square$ A $29,1 \%$
B1 20\%
B2 23,6\%
$\square$ C1 $12,7 \%$
C2 $16,4 \%$

27. Puoi indicare chi l'ha rilasciata?

28. Hai frequentato corsi di lingua italiana presso il Centro Linguistico di teneo?
$\square$ Sì $8,4 \%$
No $91,6 \%$

\section{GLI STUDI UNIVERSITARI}

29. Indica il corso di laurea cui sei iscritto:

$\square$ Laurea triennale 54\% $\square$ Laurea magistrale $26,7 \%$

$\square$ Magistrale a ciclo unico 19,3\%

30. Specifica la denominazione del tuo corso di Laurea:

\section{Indica il tuo Dipartimento:}

Ingegneria civile, edile-architettura, ambientale (DICEAA)

$8,1 \%$

Ingegneria e scienze dell'informazione e matematica (DISIM)

$8,7 \%$

Ingegneria industriale e dell'informazione e di economia (DIIIE)

$13,7 \%$ 
Medicina clinica, sanità pubblica, scienze della vita e dell'ambiente (MESVA)

Scienze cliniche applicate e biotecnologiche (DISCAB)

32. Il tuo corso di Laurea è a numero programmato?
Sì 55,3\%
$\square$ No $44,7 \%$

33. Anno al quale sei attualmente iscritto:

$\begin{array}{lr}\text { I } & 8,7 \% \\ \text { II } & 23,0 \% \\ \text { III } & 20,5 \% \\ \text { IV } & 3,7 \% \\ \text { V } & 5,0 \% \\ \text { VI } & 1,9 \% \\ \text { Fuori corso } & 37,3 \%\end{array}$

\section{Numero di CFU acquisiti:}

\section{Media dei voti:}

36. Come o dove hai ottenuto informazioni sull'Università dell'Aquila?

$\square$ Familiari/Amici 52,2\% $\square$ Ambasciata/Consolato 7,5\%

Televisione/Radio

$\square$ Giornali $0,6 \%$

$\square$ Internet $24,8 \%$

Scuola $14,9 \%$

Altro (specificare)

37. $\grave{E}$ stato facile riuscire a venire in Italia?

$\square$ Molto 16,1\% $\square$ Abbastanza 39,1\% $\square$ Poco 23\% $\square$ Per niente 21,7\%

38. Puoi raccontare brevemente la tua esperienza?

39. Quali motivi ti hanno spinto ad iscriverti all'Università dell'Aquila?

$\square$ Esenzione dalle tasse universitarie $24,8 \%$

$\square$ Il tipo e la qualità della formazione $11,8 \%$

$\square$ La presenza di accordi o progetti internazionali $5 \%$

$\square$ L'Aquila è una piccola città e si studia bene $13,7 \%$

$\square$ Vicinanza al mio luogo di residenza/Vicinanza alla mia famiglia $23,6 \%$

$\square$ Ho seguito amici/familiari $21,1 \%$

40. Frequenti le lezioni e l'ambiente universitario?

$\square$ Molto 41,6\% $\square$ Abbastanza 34,2 $\square$ Poco 15,5\% $\square$ Non frequento 8,7\% 
41. Nell'affrontare lo studio universitario, quante volte incontri le seguenti difficoltà?

\begin{tabular}{lrrcc}
\hline & Sempre & Spesso & Poco & Mai \\
\hline $\begin{array}{l}\text { Comprendere i professori quando } \\
\text { spiegano a lezione }\end{array}$ & $5,6 \%$ & $26,1 \%$ & $45,3 \%$ & $23,0 \%$ \\
\hline Comprendere i libri e i materiali didattici & $3,8 \%$ & $24,4 \%$ & $49,4 \%$ & $22,5 \%$ \\
\hline $\begin{array}{l}\text { Rispondere alle domande aperte durante } \\
\text { le prove di esame }\end{array}$ & $11,3 \%$ & $25,2 \%$ & $45,9 \%$ & $17,6 \%$ \\
$\begin{array}{l}\text { Rispondere ai test scritti durante } \\
\text { le prove di esame }\end{array}$ & $6,9 \%$ & $18,9 \%$ & $50,9 \%$ & $23,3 \%$ \\
\hline $\begin{array}{l}\text { Comprendere le domande poste dai } \\
\text { professori durante gli esami orali }\end{array}$ & $6,4 \%$ & $24,2 \%$ & $42,7 \%$ & $26,8 \%$ \\
\hline $\begin{array}{l}\text { Rispondere alle domande negli esami orali } \\
\text { nispon }\end{array}$ & $12,6 \%$ & $29,6 \%$ & $38,4 \%$ & $19,5 \%$ \\
\hline
\end{tabular}

\section{In media, quante ore al giorno dedichi allo studio per prepararti agli esami?}

\section{Quanto sei d'accordo con le affermazioni che seguono?}

\begin{tabular}{lcccc}
\hline & Molto & Abbastanza & Poco & $\begin{array}{c}\text { Per } \\
\text { niente }\end{array}$ \\
\hline $\begin{array}{l}\text { Studio per dare soddisfazione ai miei } \\
\text { familiari }\end{array}$ & $20,1 \%$ & $36,5 \%$ & $23,9 \%$ & $19,5 \%$ \\
\hline $\begin{array}{l}\text { Studio per poter accrescere il mio livello } \\
\text { sociale e culturale }\end{array}$ & $68,1 \%$ & $28,1 \%$ & $1,9 \%$ & $1,9 \%$ \\
\hline $\begin{array}{l}\text { Studio per poter trovare un lavoro ben } \\
\text { pagato }\end{array}$ & $50,0 \%$ & $37,5 \%$ & $8,8 \%$ & $3,8 \%$ \\
\hline $\begin{array}{l}\text { Lo studio è indispensabile per migliorare } \\
\text { come persona }\end{array}$ & $59,4 \%$ & $26,9 \%$ & $12,5 \%$ & $1,3 \%$ \\
\hline $\begin{array}{l}\text { Studio per poter trovare il lavoro che più } \\
\text { mi piace }\end{array}$ & $61,6 \%$ & $30,8 \%$ & $4,4 \%$ & $3,1 \%$ \\
\hline $\begin{array}{l}\text { Studio per tenermi occupato, visto che } \\
\text { ancora non riesco a trovare un lavoro }\end{array}$ & $4,4 \%$ & $14,4 \%$ & $23,8 \%$ & $57,5 \%$ \\
\hline
\end{tabular}

\section{Valuta da un minimo di 1 a un massimo di 5 la qualità dei servizi offerti dall'università:}

\begin{tabular}{lccccc}
\hline & 1 & 2 & 3 & 4 & 5 \\
\hline $\begin{array}{l}\text { Tutorato (orientamento allo studio, tutor } \\
\text { per i piani di studio...) }\end{array}$ & $29,8 \%$ & $24,8 \%$ & $25,5 \%$ & $14,3 \%$ & $5,6 \%$ \\
\hline $\begin{array}{l}\text { Servizi di segreteria (orari di } \\
\begin{array}{l}\text { apertura, efficienza, disponibilità, } \\
\text { chiarezza della modulistica...) }\end{array}\end{array}$ & $13,1 \%$ & $28,7 \%$ & $32,5 \%$ & $17,5 \%$ & $8,1 \%$ \\
\hline
\end{tabular}




\begin{tabular}{|c|c|c|c|c|c|}
\hline $\begin{array}{l}\text { Sistemi di diffusione delle informazioni } \\
\text { (guide, bacheche, siti internet...) }\end{array}$ & $15,0 \%$ & $21,9 \%$ & $30,6 \%$ & $23,1 \%$ & $9,4 \%$ \\
\hline $\begin{array}{l}\text { Qualità della didattica (disponibilità } \\
\text { docenti, regolarità lezioni, efficacia } \\
\text { dell'insegnamento) }\end{array}$ & $7,5 \%$ & $16,3 \%$ & $31,9 \%$ & $30,6 \%$ & $13,8 \%$ \\
\hline $\begin{array}{l}\text { Strutture di supporto allo studio } \\
\text { (biblioteche, videoteche, laboratori, } \\
\text { aule informatiche...) }\end{array}$ & $11,9 \%$ & $27,0 \%$ & $29,6 \%$ & $23,9 \%$ & $7,5 \%$ \\
\hline $\begin{array}{l}\text { Diritto allo studio (residenze, } \\
\text { servizio mensa, borse di studio) }\end{array}$ & $30,0 \%$ & $17,5 \%$ & $28,1 \%$ & $16,9 \%$ & $7,5 \%$ \\
\hline $\begin{array}{l}\text { Logistica (dislocazione dei dipartimenti, } \\
\text { delle segreterie, degli uffici...) }\end{array}$ & $20,8 \%$ & $27,0 \%$ & $28,9 \%$ & $16,4 \%$ & $6,9 \%$ \\
\hline $\begin{array}{l}\text { Chiarezza della modulistica e } \\
\text { delle procedure amministrative }\end{array}$ & $17,5 \%$ & $29,4 \%$ & $25,6 \%$ & $21,3 \%$ & $6,3 \%$ \\
\hline
\end{tabular}

45. Come valuti l'operato dei rappresentanti degli studenti (min 1/ $\max 5)$ ?

\begin{tabular}{|c|c|c|c|c|}
\hline 1 & 2 & 3 & 4 & 5 \\
$13,7 \%$ & $21,1 \%$ & $37,3 \%$ & $23 \%$ & $5 \%$ \\
\hline
\end{tabular}

46. Le organizzazioni studentesche quanto tengono in considerazione i problemi degli studenti stranieri?

$\square$ Molto 1,2\% $\square$ Abbastanza 24,2\% $\square$ Poco 50,9\% $\square$ Per niente 23,6\%

47. Valuta da un minimo di 1 a un massimo di 5 le azioni dell'Università dell'Aquila a favore degli studenti stranieri:

\begin{tabular}{|c|c|c|c|c|}
\hline 1 & 2 & 3 & 4 & 5 \\
$20,5 \%$ & $31,1 \%$ & $34,8 \%$ & $11,8 \%$ & $1,9 \%$ \\
\hline
\end{tabular}

48. Perché? (Esponi sinteticamente le motivazioni)

49. Hai proposte da fare per migliorare le condizioni di vita e di studio degli studenti stranieri in Univaq?

\section{LA SOCIALITÀ NELL'AMBIENTE UNIVERSITARIO}

50. Situazione sentimentale:

$\square$ Single 39,6\% $\square$ Impegnata/o 49,4\% $\square$ Non definita $11 \%$

51. Quante sono le persone che nell'ambiente universitario puoi considerare veri e propri amici?
$\square$ Nessuna $11,7 \%$
$1-236,4 \%$
3-5 $34,4 \%$
Più di 5 17,5\% 
52. Tra questi ci sono:

amici della tua stessa nazionalità

Sì 43,6\%

No $56,4 \%$

amici di nazionalità italiana

Sì $80,4 \%$

No $19,6 \%$

amici di altre nazionalità

Sì $54,6 \%$

No $45,4 \%$

53. Quante sono le persone che nell'ambiente universitario puoi considerare buoni conoscenti?

$\square$ Nessuna $4,5 \%$

$\square 1-215,6 \%$

3-5 22,1\%

$\square$ 6-10 26,6\%

$\square$ Più di 10 31,2\%

54. Tra questi ci sono:

Conoscenti della tua stessa nazionalità

Conoscenti di nazionalità italiana

$\square$ Sì $52 \% \quad \square$ No $48 \%$

Conoscenti di altre nazionalità

$\square$ Sì $92,2 \% \quad \square$ No $7,8 \%$

$\square$ Sì $72 \% \square$ No $28 \%$

55. Nel rapporto con i tuoi amici e/o conoscenti dell'ambiente universitario, che cosa ti sembra più significativo e importante?

$\square$ Studiare insieme per raggiungere gli obiettivi accademici $27,9 \%$

$\square$ Trascorrere insieme il tempo libero (cene, uscite serali, cinema, ecc.) $15,6 \%$

$\square$ Ccambiarsi informazioni, materiali, ecc. 38,3\%

Confidarsi, condividere i problemi $18,2 \%$

56. Di solito dove vi incontrate?

$\square$ A casa $46,1 \%$

$\square \mathrm{Al} \mathrm{bar/locali/pub} \mathrm{/} \mathrm{Quali?} \mathrm{31,2 \%}$

$\square$ Centri/Associazioni sportive / Quali? 8,4\%

$\square$ Centri/Associazioni culturali / Quali? 6,5\%

$\square$ In giro per la città / Dove? 35,1\%

$\square$ Negozi/Centri commerciali / Quali? 18,2\%

All'università / Dove? 92,2\%

$\square$ Centri religiosi/luoghi di culto / Quali? 2,6\%

Social network: Facebook, Whatsapp, Skype, ecc. 58,4\%

57. Appartieni o collabori con qualche associazione/organizzazione che opera in ambito sociale e culturale?

$\square$ Sì $17,5 \% \quad \square$ No $82,5 \%$

58. Se sì, quale?

59. Secondo te qual è la percentuale di studenti stranieri che studiano all'Università dell'Aquila? In media $16 \%$ 


\section{Quanto sei d'accordo con le seguenti affermazioni?}

\begin{tabular}{lrrrr}
\hline $\begin{array}{l}\text { I miei colleghi italiani.../ } \\
\text { le mie colleghe italiane... }\end{array}$ & Molto & Abbastanza & Poco & $\begin{array}{c}\text { Per } \\
\text { niente }\end{array}$ \\
\hline Sono disponibili al confronto e all'aiuto & $24,7 \%$ & $53,2 \%$ & $16,9 \%$ & $5,2 \%$ \\
\hline $\begin{array}{l}\text { Sono aperti e curiosi nei confronti } \\
\text { delle altre culture }\end{array}$ & $20,9 \%$ & $32,0 \%$ & $36,6 \%$ & $10,5 \%$ \\
\hline $\begin{array}{l}\text { Sanno condividere le esperienze } \\
\text { personali }\end{array}$ & $16,4 \%$ & $43,4 \%$ & $34,2 \%$ & $5,9 \%$ \\
\hline Sono sensibili ai problemi degli altri & $11,8 \%$ & $43,4 \%$ & $34,9 \%$ & $9,9 \%$ \\
\hline Hanno sensibilità per i problemi sociali & $9,2 \%$ & $45,4 \%$ & $35,5 \%$ & $9,9 \%$ \\
\hline Sono disponibili all'amicizia & $22,9 \%$ & $45,8 \%$ & $25,5 \%$ & $5,9 \%$ \\
\hline $\begin{array}{l}\text { Sanno condividere le esperienze } \\
\text { nello studio }\end{array}$ & $17,1 \%$ & $50,0 \%$ & $29,6 \%$ & $3,3 \%$ \\
\hline $\begin{array}{l}\text { Si impegnano per ottenere buoni } \\
\text { risultati accademici }\end{array}$ & $23,0 \%$ & $59,2 \%$ & $15,8 \%$ & $2,0 \%$ \\
\hline $\begin{array}{l}\text { Partecipano attivamente alle iniziative } \\
\text { di carattere sociale }\end{array}$ & $7,9 \%$ & $32,9 \%$ & $50,0 \%$ & $9,2 \%$ \\
\hline $\begin{array}{l}\text { Partecipano attivamente alle iniziative per } \\
\text { il miglioramento della realtà universitaria }\end{array}$ & $5,9 \%$ & $27,6 \%$ & $52,6 \%$ & $13,8 \%$ \\
\hline $\begin{array}{l}\text { Rispettano le diversità culturali } \\
\text { Rispettano le diversità religiose }\end{array}$ & $19,1 \%$ & $52,6 \%$ & $23,0 \%$ & $5,3 \%$ \\
\hline Accettano di buon grado amici stranieri & $22,4 \%$ & $59,1 \%$ & $17,4 \%$ & $4,7 \%$ \\
\hline \begin{tabular}{l} 
Sono solidali \\
\hline
\end{tabular} & $16,1 \%$ & $49,0 \%$ & $27,0 \%$ & $4,6 \%$ \\
\hline
\end{tabular}

\section{Quanto sei d'accordo con le seguenti affermazioni?}

\begin{tabular}{|c|c|c|c|c|}
\hline $\begin{array}{l}\text { I miei colleghi stranieri.../ } \\
\text { le mie colleghe straniere... }\end{array}$ & Molto & Abbastanza & Poco & $\begin{array}{l}\text { Per } \\
\text { niente }\end{array}$ \\
\hline Sono disponibili al confronto e all'aiuto & $22,1 \%$ & $63,0 \%$ & $12,3 \%$ & $2,6 \%$ \\
\hline $\begin{array}{l}\text { Sono aperti e curiosi nei confronti } \\
\text { delle altre culture }\end{array}$ & $23,5 \%$ & $57,5 \%$ & $15,0 \%$ & $3,9 \%$ \\
\hline $\begin{array}{l}\text { Sanno condividere le esperienze } \\
\text { personali }\end{array}$ & $18,3 \%$ & $54,9 \%$ & $24,2 \%$ & $2,6 \%$ \\
\hline Sono sensibili ai problemi degli altri & $13,1 \%$ & $56,9 \%$ & $26,1 \%$ & $3,9 \%$ \\
\hline Hanno sensibilità per i problemi sociali & $17,2 \%$ & $54,3 \%$ & $25,2 \%$ & $3,3 \%$ \\
\hline Sono disponibili all'amicizia & $25,0 \%$ & $60,5 \%$ & $11,8 \%$ & $2,6 \%$ \\
\hline $\begin{array}{l}\text { Sanno condividere le esperienze } \\
\text { nello studio }\end{array}$ & $20,0 \%$ & $56,0 \%$ & $20,7 \%$ & $3,3 \%$ \\
\hline $\begin{array}{l}\text { Si impegnano per ottenere buoni } \\
\text { risultati accademici }\end{array}$ & $27,2 \%$ & $56,3 \%$ & $13,9 \%$ & $2,6 \%$ \\
\hline $\begin{array}{l}\text { Partecipano attivamente alle iniziative } \\
\text { di carattere sociale }\end{array}$ & $9,3 \%$ & $39,1 \%$ & $44,4 \%$ & $7,3 \%$ \\
\hline
\end{tabular}




\begin{tabular}{lcccc}
\hline $\begin{array}{l}\text { Partecipano attivamente alle iniziative per } \\
\text { il miglioramento della realtà universitaria }\end{array}$ & $5,3 \%$ & $31,8 \%$ & $51,7 \%$ & $11,3 \%$ \\
\hline Rispettano le diversità culturali & $29,8 \%$ & $57,0 \%$ & $10,6 \%$ & $2,6 \%$ \\
\hline Rispettano le diversità religiose & $29,8 \%$ & $55,0 \%$ & $12,6 \%$ & $2,6 \%$ \\
\hline Accettano di buon grado amici italiani & $30,9 \%$ & $55,7 \%$ & $10,7 \%$ & $2,7 \%$ \\
\hline Sono solidali & $23,5 \%$ & $59,7 \%$ & $14,1 \%$ & $2,7 \%$ \\
\hline
\end{tabular}

62. Ti sei mai sentito colpito da forme di pregiudizio nell'ambiente accademico?
$\square$ Sì $53,2 \%$
$\square$ No $46,8 \%$

\section{Se Sì, da parte di chi?}

$\square$ Altri studenti $69,3 \% \quad \square$ Docenti $56 \% \quad \square$ Personale amministrativo 25,3\%

64. Puoi raccontare brevemente?

\section{LA VITA IN ITALIA E ALL'AQUILA}

65. Come valuti su una scala da 1 a 5 i seguenti aspetti della società e della cultura italiana?

\begin{tabular}{lrrrrr}
\hline & 1 & 2 & 3 & 4 & \multicolumn{1}{c}{5} \\
\hline Abitudini alimentari & $3,3 \%$ & $6,6 \%$ & $18,5 \%$ & $32,5 \%$ & $39,1 \%$ \\
\hline Sistema di istruzione & $3,9 \%$ & $21,1 \%$ & $42,1 \%$ & $27,0 \%$ & $5,9 \%$ \\
\hline $\begin{array}{l}\text { Apertura e disponibilità verso } \\
\text { altre culture e religioni }\end{array}$ & $10,6 \%$ & $31,1 \%$ & $26,5 \%$ & $22,5 \%$ & $9,3 \%$ \\
\hline Musica & $3,3 \%$ & $11,3 \%$ & $29,8 \%$ & $33,8 \%$ & $21,9 \%$ \\
\hline Cinema, teatro & $6,0 \%$ & $10,6 \%$ & $30,5 \%$ & $33,8 \%$ & $19,2 \%$ \\
\hline Programmi televisivi & $12,7 \%$ & $26,0 \%$ & $31,3 \%$ & $22,0 \%$ & $8,0 \%$ \\
\hline Sistema socio-economico & $13,2 \%$ & $29,1 \%$ & $40,4 \%$ & $14,6 \%$ & $2,6 \%$ \\
\hline Sport & $2,0 \%$ & $8,7 \%$ & $32,7 \%$ & $34,7 \%$ & $22,0 \%$ \\
\hline Sistema politico & $40,7 \%$ & $28,7 \%$ & $20,7 \%$ & $6,7 \%$ & $3,3 \%$ \\
\hline Sistema sanitario & $6,0 \%$ & $18,7 \%$ & $32,0 \%$ & $34,7 \%$ & $8,7 \%$ \\
\hline Territorio e ambiente & $6,6 \%$ & $16,6 \%$ & $30,5 \%$ & $29,1 \%$ & $17,2 \%$ \\
\hline
\end{tabular}

66. Conosci all'Aquila luoghi dedicati alla cultura del tuo Paese (ristoranti, alimentari, circoli e associazioni, luoghi di culto, luoghi di incontro, ecc.)?

$\square$ Sì $16,45 \%$

Quali?

$\square$ No $83,55 \%$ 


\section{Il tuo Paese di origine:}

Mi manca tanto che voglio tornare a viverci $21,7 \%$

$\square$ Mi manca, ma voglio costruire il mio futuro altrove $48,7 \%$

$\square$ Sono indeciso se tornare a viverci $17,1 \%$

$\square$ Non mi manca affatto $12,5 \%$

\section{Pensi di rimanere a vivere in Italia?}

$\square$ Sì, perché $21,7 \%$

$\square$ No, perché $24,3 \%$

$\square$ Non so, perché $53,9 \%$

70. Come valuti, su una scala da 1 a 5 , la città dell'Aquila rispetto ai seguenti aspetti?

\begin{tabular}{|c|c|c|c|c|c|}
\hline & 1 & 2 & 3 & 4 & 5 \\
\hline $\begin{array}{l}\text { Condizioni abitative generali } \\
\text { (qualità, costi e reperibilità } \\
\text { degli alloggi) }\end{array}$ & $32,5 \%$ & $31,8 \%$ & $27,2 \%$ & $5,3 \%$ & $3,3 \%$ \\
\hline Prospettive occupazionali & $33,3 \%$ & $44,2 \%$ & $16,3 \%$ & $4,8 \%$ & $1,4 \%$ \\
\hline Servizi sociosanitari & $7,6 \%$ & $28,3 \%$ & $41,4 \%$ & $18,6 \%$ & $4,1 \%$ \\
\hline $\begin{array}{l}\text { Relazioni interpersonali e vita } \\
\text { sociale }\end{array}$ & $11,4 \%$ & $34,2 \%$ & $32,2 \%$ & $16,8 \%$ & $5,4 \%$ \\
\hline $\begin{array}{l}\text { Servizi per il pubblico offerti } \\
\text { da uffici e istituzioni }\end{array}$ & $17,1 \%$ & $32,9 \%$ & $32,9 \%$ & $15,1 \%$ & $2,1 \%$ \\
\hline Intrattenimento e svago & $30,4 \%$ & $33,1 \%$ & $23,0 \%$ & $10,1 \%$ & $3,4 \%$ \\
\hline Stimoli culturali & $19,7 \%$ & $40,1 \%$ & $26,5 \%$ & $11,6 \%$ & $2,0 \%$ \\
\hline $\begin{array}{l}\text { Spazi per il tempo libero } \\
\text { e luoghi di aggregazione }\end{array}$ & $26,0 \%$ & $40,4 \%$ & $23,3 \%$ & $8,9 \%$ & $1,4 \%$ \\
\hline $\begin{array}{l}\text { Trasporti e mobilità sul territorio } \\
\text { urbano }\end{array}$ & $33,3 \%$ & $31,3 \%$ & $21,3 \%$ & $11,3 \%$ & $2,7 \%$ \\
\hline $\begin{array}{l}\text { Assetto urbano (nuovi } \\
\text { agglomerati, situazione } \\
\text { del centro storico...) }\end{array}$ & $29,7 \%$ & $35,8 \%$ & $26,4 \%$ & $6,1 \%$ & $2,0 \%$ \\
\hline Sicurezza & $7,5 \%$ & $23,1 \%$ & $30,6 \%$ & $26,5 \%$ & $12,2 \%$ \\
\hline Qualità del paesaggio & $6,1 \%$ & $12,9 \%$ & $29,9 \%$ & $28,6 \%$ & $22,4 \%$ \\
\hline Qualità del territorio & $4,8 \%$ & $19,7 \%$ & $34,7 \%$ & $28,6 \%$ & $12,2 \%$ \\
\hline Vita politica & $34,9 \%$ & $27,4 \%$ & $30,1 \%$ & $4,1 \%$ & $3,4 \%$ \\
\hline Luoghi di culto e spiritualità & $19,0 \%$ & $22,4 \%$ & $33,3 \%$ & $15,6 \%$ & $9,5 \%$ \\
\hline Qualità della vita & $13,3 \%$ & $30,0 \%$ & $40,0 \%$ & $14,7 \%$ & $2,0 \%$ \\
\hline
\end{tabular}


71. Nella tua esperienza di studio all'Aquila, quanto ti senti coinvolto nelle vicende legate ai problemi della ricostruzione? (da un minimo di 1 a un massimo di 5)

\begin{tabular}{|c|c|c|c|c|}
\hline 1 & 2 & 3 & 4 & 5 \\
$30,3 \%$ & $19,7 \%$ & $27 \%$ & $13,2 \%$ & $9,9 \%$ \\
\hline
\end{tabular}

72. Vuoi aggiungere qualcosa?

GRAZIE PER LA COLLABORAZIONE! 


\section{QUESTIONARIO STUDENTI ITALIANI}

Garantiamo l'assoluto anonimato e la riservatezza delle informazioni che ci saranno fornite nel rispetto della normativa sulla privacy

\section{DATI GENERALI}
1. Sesso:
Maschio 44,9\%
Femmina 55,1\%

2. Età:

3. Luogo di nascita:

4. Cittadinanza:
5. Religione:
$\square$ Nessuna $24,3 \%$
Musulmana
Cristiana 70,2\%
$\square$ Ebraica $0,3 \%$
Buddista $1,3 \%$
Induista
$\square$ Altro $3,5 \%$

6. Sei un credente praticante?

Molto $11,3 \%$

Abbastanza 27,2\%

$\square$ Poco $38,5 \% \quad \square$ Per niente $22,9 \%$

7. Comune di residenza:

8. Comune di domicilio: $\square$ L'Aquila $\square$ Altro comune (specificare)

9. Dove vive la tua famiglia?

Nazione Città

10. Per frequentare l'università:

$\square$ Vivo con altri studenti in una casa in affitto (indicare quartiere o frazione) $35,9 \%$

$\square$ Vivo con altri studenti (in una residenza universitaria) 2,5\%

$\square$ Viaggio $38,2 \%$

$\square$ Risiedo all'Aquila con la mia famiglia $17,6 \%$

$\square$ Altro: $5,8 \%$

11. Se vivi con altri studenti, puoi dirci se ci sono stranieri tra di loro?

$\square$ Sì $12,1 \%$

$\square$ No $87,9 \%$ 
12. Titolo di studio dei genitori (indica quello corrispondente al titolo italiano)

\begin{tabular}{lcccccc}
\hline & Nessuno & $\begin{array}{c}\text { Scuola } \\
\text { Elementare }\end{array}$ & $\begin{array}{c}\text { Scuola } \\
\text { Media }\end{array}$ & $\begin{array}{c}\text { Qualifica } \\
\text { professionale }\end{array}$ & $\begin{array}{c}\text { Scuola } \\
\text { Superiore }\end{array}$ & Laurea \\
\hline Madre & $\square$ & $7,3 \%$ & $23,9 \%$ & $8,8 \%$ & $41,5 \%$ & $18,6 \%$ \\
\hline Padre & $\square$ & $5,8 \%$ & $26,4 \%$ & $12,6 \%$ & $40,2 \%$ & $15,1 \%$ \\
\hline
\end{tabular}

13. Puoi indicare la fascia di reddito della tua famiglia in Italia/nel tuo Paese?

$\square$ Bassa $12,8 \% \quad \square$ Medio/bassa $28,9 \% \quad \square$ Media $48 \%$

\section{Chi paga i tuoi studi?}

$\square$ La mia famiglia $57 \%$

$\square$ Li pago da solo $19,3 \%$

$\square$ La mia famiglia mi aiuta, ma cerco di contribuire personalmente $19,3 \%$

$\square$ Ho una borsa di studio italiana $4 \%$

$\square$ Altro $0,3 \%$

\section{Svolgi qualche attività lavorativa?}

$\square$ Sì, lavoro 21,4\% $\square$ Sì, lavoro ma saltuariamente 30,2\%

$\square$ No, studio soltanto $48,6 \%$

\section{LA SCUOLA}

\section{Scuola superiore frequentata}

$\square$ Liceo ad indirizzo umanistico e/o scientifico $66,8 \%$

$\square$ Istituto tecnico-Istituto professionale $33,2 \%$

Specificare:

17. Voto finale del diploma di scuola superiore:

\begin{tabular}{llll}
\hline $60-70$ & $71-80$ & $81-90$ & $91-100$ \\
\hline $27,6 \%$ & $27,1 \%$ & $22,6 \%$ & $21,6 \%$ \\
\hline
\end{tabular}

Non è previsto il voto finale: $1 \%$ 
18. In base alle tue esperienze dirette o indirette, la scuola italiana quanto è in grado di supportare i ragazzi stranieri nelle seguenti aree?

\begin{tabular}{lcccc}
\hline & Molto & Abbastanza & Poco & $\begin{array}{c}\text { Per } \\
\text { niente }\end{array}$ \\
\hline Lingua italiana & $9,8 \%$ & $52,0 \%$ & $35,9 \%$ & $2,3 \%$ \\
\hline Lingue straniere & $1,8 \%$ & $37,3 \%$ & $51,6 \%$ & $9,3 \%$ \\
\hline $\begin{array}{l}\text { Apprendimenti nelle discipline } \\
\text { umanistiche }\end{array}$ & $6,4 \%$ & $49,4 \%$ & $41,2 \%$ & $3,1 \%$ \\
$\begin{array}{l}\text { Apprendimenti nelle discipline } \\
\text { tecnico-scientifiche }\end{array}$ & $6,9 \%$ & $54,7 \%$ & $35,1 \%$ & $3,3 \%$ \\
\hline $\begin{array}{l}\text { Attività artistiche e/o sportive } \\
\text { Integrazione sociale e benessere }\end{array}$ & $10,4 \%$ & $52,4 \%$ & $30,6 \%$ & $6,6 \%$ \\
\hline scolastico & $4,8 \%$ & $40,9 \%$ & $45,4 \%$ & $8,9 \%$ \\
\hline
\end{tabular}

\section{GLI STUDI UNIVERSITARI}

19. Indica il corso di laurea cui sei iscritto:

$\square$ Laurea triennale 54,3\% $\square$ Laurea magistrale $34,4 \%$

Magistrale a ciclo unico 11,3\%

20. Specifica la denominazione del tuo corso di Laurea:

\section{Indica il tuo Dipartimento:}

Ingegneria civile, edile-architettura, ambientale (DICEAA) $6,5 \%$ Ingegneria e scienze dell'informazione e matematica (DISIM) $\quad 7,3 \%$ Ingegneria industriale e dell'informazione e di economia (DIIIE) 21,9\% Medicina clinica, sanità pubblica, scienze della vita e dell'ambiente (MESVA)

Scienze cliniche applicate e biotecnologiche (DISCAB) $5,5 \%$

Scienze fisiche e chimiche (DSFC) $1,8 \%$

Scienze umane (DSU)

\section{Il tuo corso di Laurea è a numero programmato?}
$\square$ Sì $29,4 \%$
$\square$ No $70,6 \%$ 
23. Anno al quale sei attualmente iscritto:

$\begin{array}{lr}\text { I } & 4,8 \% \\ \text { II } & 30,9 \% \\ \text { III } & 21,1 \% \\ \text { IV } & 6,8 \% \\ \text { V } & 2,3 \% \\ \text { VI } & 2,5 \% \\ \text { Fuori corso } & 31,7 \%\end{array}$

24. Numero di CFU acquisiti:

25. Media dei voti:

26. Come o dove hai ottenuto informazioni sull'Università dell'Aquila?

$\square$ Familiari/Amici 51\% $\square$ Ambasciata/Consolato $\square$ Televisione/Radio

$\square$ Giornali $\square$ Internet 30,4\% $\square$ Scuola 16,1\% $\square$ Altro (specificare) 2,5\%

27. Quali motivi principali ti hanno spinto ad iscriverti all'Università dell'Aquila?

$\square$ Esenzione dalle tasse universitarie 22,9\%

$\square$ Il tipo e la qualità della formazione $14,6 \%$

$\square$ La presenza di accordi o progetti internazionali $0,3 \%$

$\square$ L'Aquila è una piccola città e si studia bene 13,3\%

$\square$ Vicinanza al mio luogo di residenza/Vicinanza alla mia famiglia 35,9\%

$\square$ Ho seguito amici/familiari $6 \%$

$\square$ Altro $7 \%$

28. Frequenti le lezioni e l'ambiente universitario?

Molto 36,9\% $\square$ Abbastanza 30,2\%

$\square$ Poco $18,3 \% \quad \square$ Non frequento $14,6 \%$

29. Nell'affrontare lo studio universitario, quante volte incontri le seguenti difficoltà?

\begin{tabular}{|c|c|c|c|c|}
\hline & Sempre & Spesso & Poco & Mai \\
\hline $\begin{array}{l}\text { Comprendere i professori quando } \\
\text { spiegano a lezione }\end{array}$ & $2,3 \%$ & $19,7 \%$ & $56,5 \%$ & $21,5 \%$ \\
\hline Comprendere i libri e i materiali didattici & $2,5 \%$ & $18,9 \%$ & $61,7 \%$ & $16,9 \%$ \\
\hline $\begin{array}{l}\text { Rispondere alle domande aperte } \\
\text { durante le prove di esame }\end{array}$ & $2,3 \%$ & $15,4 \%$ & $58,7 \%$ & $23,6 \%$ \\
\hline
\end{tabular}




\begin{tabular}{lcccc}
\hline $\begin{array}{l}\text { Rispondere ai test scritti durante } \\
\text { le prove di esame }\end{array}$ & $2,3 \%$ & $17,6 \%$ & $59,7 \%$ & $20,4 \%$ \\
\hline $\begin{array}{l}\text { Comprendere le domande poste dai } \\
\text { professori durante gli esami orali }\end{array}$ & $2,0 \%$ & $15,3 \%$ & $54,7 \%$ & $27,9 \%$ \\
\hline \begin{tabular}{l} 
Rispondere alle domande negli esami orali \\
\hline
\end{tabular} & $2,6 \%$ & $15,8 \%$ & $58,0 \%$ & $23,6 \%$ \\
\hline
\end{tabular}

30. In media, quante ore al giorno dedichi allo studio per prepararti agli esami?

\section{Quanto sei d'accordo con le affermazioni che seguono?}

\begin{tabular}{lcccc}
\hline & Molto & Abbastanza & Poco & $\begin{array}{c}\text { Per } \\
\text { niente }\end{array}$ \\
\hline $\begin{array}{l}\text { Studio per dare soddisfazione } \\
\text { ai miei familiari }\end{array}$ & $14,2 \%$ & $33,3 \%$ & $31,8 \%$ & $20,6 \%$ \\
\hline $\begin{array}{l}\text { Studio per poter accrescere il mio } \\
\text { livello sociale e culturale }\end{array}$ & $65,0 \%$ & $28,0 \%$ & $6,0 \%$ & $1,0 \%$ \\
\hline $\begin{array}{l}\text { Studio per poter trovare un lavoro } \\
\text { ben pagato }\end{array}$ & $35,0 \%$ & $42,2 \%$ & $16,9 \%$ & $5,9 \%$ \\
\hline $\begin{array}{l}\text { Lo studio è indispensabile per } \\
\text { migliorare come persona }\end{array}$ & $53,1 \%$ & $33,5 \%$ & $9,1 \%$ & $4,3 \%$ \\
\hline $\begin{array}{l}\text { Studio per poter trovare il lavoro che } \\
\text { più mi piace }\end{array}$ & $60,7 \%$ & $26,8 \%$ & $7,9 \%$ & $4,6 \%$ \\
\hline $\begin{array}{l}\text { Studio per tenermi occupato, visto che } \\
\text { ancora non riesco a trovare un lavoro }\end{array}$ & $3,3 \%$ & $5,4 \%$ & $26,3 \%$ & $65,1 \%$ \\
\hline
\end{tabular}

\section{Valuta da un minimo di 1 a un massimo di 5 la qualità dei servizi offerti dall'università:}

\begin{tabular}{lccccc}
\hline & 1 & 2 & 3 & 4 & 5 \\
\hline $\begin{array}{l}\text { Tutorato (orientamento allo studio, tutor } \\
\text { per i piani di studio...) }\end{array}$ & $34,2 \%$ & $26,0 \%$ & $30,1 \%$ & $8,7 \%$ & $1,0 \%$ \\
\hline $\begin{array}{l}\text { Servizi di segreteria (orari di } \\
\text { apertura, efficienza, disponibilità, } \\
\text { chiarezza della modulistica...) }\end{array}$ & $22,7 \%$ & $30,0 \%$ & $28,0 \%$ & $14,9 \%$ & $4,5 \%$ \\
\hline $\begin{array}{l}\text { Sistemi di diffusione delle } \\
\text { informazioni (guide, bacheche, siti } \\
\text { internet...) }\end{array}$ & $15,2 \%$ & $26,8 \%$ & $35,9 \%$ & $18,5 \%$ & $3,5 \%$ \\
\hline $\begin{array}{l}\text { Qualità della didattica (disponibilità } \\
\text { docenti, regolarità lezioni, efficacia } \\
\text { dell'insegnamento) }\end{array}$ & $7,1 \%$ & $14,4 \%$ & $31,3 \%$ & $36,9 \%$ & $10,4 \%$ \\
\hline $\begin{array}{l}\text { Strutture di supporto allo studio } \\
\text { (biblioteche, videoteche, laboratori, } \\
\text { aule informatiche...) }\end{array}$ & $20,4 \%$ & $29,3 \%$ & $30,0 \%$ & $18,3 \%$ & $2,0 \%$ \\
\hline
\end{tabular}


Diritto allo studio (residenze,

$31,9 \% \quad 32,4 \% \quad 23,7 \% \quad 10,5 \% \quad 1,5 \%$

servizio mensa, borse di studio)

Logistica (dislocazione dei

$24,5 \% \quad 29,6 \% \quad 31,1 \% \quad 11,7 \% \quad 3,1 \%$

dipartimenti, delle segreterie,

degli uffici...)

Chiarezza della modulistica

$22,3 \% \quad 30,6 \% \quad 30,1 \% \quad 12,9 \% \quad 4,1 \%$

e delle procedure amministrative

33. Come valuti l'operato dei rappresentanti degli studenti (min 1/ $\max 5)$ ?

\begin{tabular}{|c|c|c|c|c|}
\hline 1 & 2 & 3 & 4 & 5 \\
$12,8 \%$ & $14,6 \%$ & $40,5 \%$ & $24,6 \%$ & $7,5 \%$ \\
\hline
\end{tabular}

34. Le organizzazioni studentesche quanto tengono in considerazione $i$ problemi degli studenti stranieri?

$\square$ Molto 5,8\% $\square$ Abbastanza 52,8\% $\square$ Poco 35,2\% $\square$ Per niente 6,3\%

35. Valuta da un minimo di 1 a un massimo di 5 le azioni dell'Università dell'Aquila a favore degli studenti stranieri:

\begin{tabular}{|c|c|c|c|c|}
\hline 1 & 2 & 3 & 4 & 5 \\
$10,8 \%$ & $19,3 \%$ & $49 \%$ & $17,8 \%$ & $3 \%$ \\
\hline
\end{tabular}

36. Perché? (Esponi sinteticamente le motivazioni)

37. Hai proposte da fare per migliorare le condizioni di vita e di studio degli studenti stranieri in Univaq?

\section{LA SOCIALITÀ NELL'AMBIENTE UNIVERSITARIO}

38. Situazione sentimentale:

$\square$ Single $31,2 \% \quad \square$ Impegnata/o 61,1\% $\square$ Non definita $7,8 \%$

39. Quante sono le persone che nell'ambiente universitario puoi considerare veri e propri amici?

$\square$ Nessuna $12,6 \% \quad \square 1-232,4 \% \quad \square 3-538,2 \% \quad \square$ Più di $516,8 \%$

40. Tra questi ci sono:

Amici di nazionalità italiana $\quad \square$ Sì 91,9\% $\square$ No 8,1\%

Amici di altre nazionalità

$\square$ Sì $16,3 \% \quad \square$ No $83,7 \%$ 
41. Oltre ai tuoi amici, quante sono le persone che nell'ambiente universitario puoi considerare buoni conoscenti?

$\square$ Nessuna $3,8 \%$

$\square 1-210,6 \%$

$3-523,6 \%$

6-10 22,4\%

$\square$ Più di 10 39,7\%

42. Tra questi ci sono:

Conoscenti di nazionalità italiana

$\square$ Sì $97,2 \% \quad \square$ No 2,8\%

Conoscenti di altre nazionalità

Sì $38,6 \% \square$ No $61,4 \%$

43. Nel rapporto con i tuoi amici e/o conoscenti dell'ambiente universitario, che cosa ti sembra più significativo e importante?

$\square$ Studiare insieme per raggiungere gli obiettivi accademici 23,1\%

Trascorrere insieme il tempo libero (cene, uscite serali, cinema, ecc.) 20,9\%

Scambiarsi informazioni, materiali, ecc. $41 \%$

Confidarsi, condividere i problemi $15,1 \%$

44. Di solito dove vi incontrate?
A casa $32,2 \%$
$\square \mathrm{Al}$ bar/locali/pub 30,4\%
Centri/associazioni sportive 3,5\%
In giro per la città $23,9 \%$
$\square$ Centri/associazioni culturali 4,3\%
All'università $82,7 \%$
$\square$ Negozi/centri commerciali $6,5 \%$
Social network: Facebook, Whatsapp, Skype, ecc. 33,9\%

45. Appartieni o collabori con qualche associazione/organizzazione che opera in ambito sociale e culturale?

Sì $19,8 \%$

$\square$ No $80,2 \%$

46. Se sì, quale?

47. Secondo te qual è la percentuale di stranieri residenti in Italia sul totale della popolazione?

48. Secondo te qual è la percentuale di studenti stranieri che studiano all'Università dell'Aquila?

In media $11,5 \%$ 


\section{Quanto sei d'accordo con le seguenti affermazioni?}

\begin{tabular}{lrrrr}
\hline $\begin{array}{l}\text { I miei colleghi italiani.../ } \\
\text { le mie colleghe italiane... }\end{array}$ & Molto & Abbastanza & Poco & $\begin{array}{c}\text { Per } \\
\text { niente }\end{array}$ \\
\hline Sono disponibili al confronto e all'aiuto & $11,6 \%$ & $64,7 \%$ & $22,4 \%$ & $1,3 \%$ \\
\hline $\begin{array}{l}\text { Sono aperti e curiosi nei confronti } \\
\text { delle altre culture }\end{array}$ & $7,3 \%$ & $50,5 \%$ & $39,2 \%$ & $3,0 \%$ \\
\hline $\begin{array}{l}\text { Sanno condividere le esperienze } \\
\text { personali }\end{array}$ & $8,4 \%$ & $55,8 \%$ & $34,3 \%$ & $1,5 \%$ \\
\hline Sono sensibili ai problemi degli altri & $7,1 \%$ & $48,6 \%$ & $38,3 \%$ & $6,0 \%$ \\
\hline Hanno sensibilità per i problemi sociali & $5,5 \%$ & $48,1 \%$ & $42,1 \%$ & $4,3 \%$ \\
\hline Sono disponibili all'amicizia & $15,7 \%$ & $64,9 \%$ & $18,4 \%$ & $1,0 \%$ \\
\hline $\begin{array}{l}\text { Sanno condividere le esperienze } \\
\text { nello studio }\end{array}$ & $12,9 \%$ & $58,7 \%$ & $25,1 \%$ & $3,3 \%$ \\
\hline $\begin{array}{l}\text { Si impegnano per ottenere buoni } \\
\text { risultati accademici }\end{array}$ & $17,8 \%$ & $69,3 \%$ & $12,1 \%$ & $0,8 \%$ \\
\hline $\begin{array}{l}\text { Partecipano attivamente alle iniziative } \\
\text { di carattere sociale }\end{array}$ & $2,5 \%$ & $29,0 \%$ & $60,5 \%$ & $8,1 \%$ \\
\hline $\begin{array}{l}\text { Partecipano attivamente alle iniziative } \\
\text { per il miglioramento della realtà } \\
\text { universitaria }\end{array}$ & $2,8 \%$ & $25,6 \%$ & $57,0 \%$ & $14,7 \%$ \\
\hline Rispettano le diversità culturali & $15,6 \%$ & $63,7 \%$ & $18,1 \%$ & $2,5 \%$ \\
\hline $\begin{array}{l}\text { Rispettano le diversità religiose } \\
\text { Accettano di buon grado amici stranieri }\end{array}$ & $14,7 \%$ & $62,0 \%$ & $20,0 \%$ & $3,3 \%$ \\
\hline \begin{tabular}{l} 
Sono solidali \\
\hline
\end{tabular} & $12,1 \%$ & $65,7 \%$ & $17,2 \%$ & $1,3 \%$ \\
\hline
\end{tabular}

\section{Quanto sei d'accordo con le seguenti affermazioni?}

\begin{tabular}{|c|c|c|c|c|}
\hline $\begin{array}{l}\text { I miei colleghi stranieri.../ } \\
\text { le mie colleghe straniere... }\end{array}$ & Molto & Abbastanza & Poco & $\begin{array}{l}\text { Per } \\
\text { niente }\end{array}$ \\
\hline Sono disponibili al confronto e all'aiuto & $12,8 \%$ & $68,6 \%$ & $16,6 \%$ & $2,0 \%$ \\
\hline $\begin{array}{l}\text { Sono aperti e curiosi nei confronti delle } \\
\text { altre culture }\end{array}$ & $15,4 \%$ & $63,4 \%$ & $18,9 \%$ & $2,3 \%$ \\
\hline $\begin{array}{l}\text { Sanno condividere le esperienze } \\
\text { personali }\end{array}$ & $11,7 \%$ & $60,4 \%$ & $25,9 \%$ & $2,0 \%$ \\
\hline Sono sensibili ai problemi degli altri & $8,9 \%$ & $60,1 \%$ & $28,8 \%$ & $2,3 \%$ \\
\hline Hanno sensibilità per i problemi sociali & $7,9 \%$ & $57,3 \%$ & $30,8 \%$ & $4,1 \%$ \\
\hline Sono disponibili all'amicizia & $19,3 \%$ & $63,4 \%$ & $15,5 \%$ & $1,8 \%$ \\
\hline $\begin{array}{l}\text { Sanno condividere le esperienze nello } \\
\text { studio }\end{array}$ & $10,0 \%$ & $64,8 \%$ & $22,1 \%$ & $3,1 \%$ \\
\hline $\begin{array}{l}\text { Si impegnano per ottenere buoni } \\
\text { risultati accademici }\end{array}$ & $17,6 \%$ & $67,1 \%$ & $13,8 \%$ & $1,5 \%$ \\
\hline $\begin{array}{l}\text { Partecipano attivamente alle iniziative di } \\
\text { carattere sociale }\end{array}$ & $5,4 \%$ & $46,4 \%$ & $41,3 \%$ & $6,9 \%$ \\
\hline
\end{tabular}


\begin{tabular}{lllll}
\hline Partecipano attivamente alle iniziative & $3,6 \%$ & $36,6 \%$ & $47,3 \%$ & $12,5 \%$
\end{tabular} per il miglioramento della realtà

universitaria

\begin{tabular}{lllll}
\hline Rispettano le diversità culturali & $15,8 \%$ & $64,5 \%$ & $16,6 \%$ & $3,1 \%$ \\
\hline Rispettano le diversità religiose & $14,8 \%$ & $61,9 \%$ & $19,4 \%$ & $3,8 \%$ \\
\hline Accettano di buon grado amici italiani & $21,3 \%$ & $64,8 \%$ & $12,1 \%$ & $1,8 \%$ \\
\hline Sono solidali & $14,1 \%$ & $66,1 \%$ & $17,2 \%$ & $2,6 \%$ \\
\hline
\end{tabular}

51. Pensi che ci siano forme di pregiudizio nei confronti degli studenti stranieri nell'ambiente universitario?

$\square$ Sì $25,1 \%$

$\square$ No $74,9 \%$

52. Se Sì, da parte di chi?

$\square$ Altri studenti $87,1 \% \quad \square$ Docenti $31,7 \%$

$\square$ Personale amministrativo $15,8 \% \quad \square$ Altro $4 \%$

53. Puoi raccontare brevemente?

\section{LA VITA IN ITALIA E ALL'AQUILA}

54. Valuta i seguenti aspetti della società e della cultura italiana su una scala da 1 a 5

\begin{tabular}{lrrrrr}
\hline & 1 & 2 & 3 & 4 & 5 \\
\hline Abitudini alimentari & $3,4 \%$ & $6,0 \%$ & $20,2 \%$ & $34,2 \%$ & $36,3 \%$ \\
\hline Sistema di istruzione & $11,3 \%$ & $24,5 \%$ & $43,6 \%$ & $18,0 \%$ & $2,6 \%$ \\
\hline $\begin{array}{l}\text { Apertura e disponibilità verso } \\
\text { altre culture e religioni }\end{array}$ & $11,6 \%$ & $26,9 \%$ & $44,2 \%$ & $14,0 \%$ & $3,4 \%$ \\
\hline Musica & $3,4 \%$ & $18,3 \%$ & $33,5 \%$ & $30,7 \%$ & $14,2 \%$ \\
\hline Cinema, teatro & $3,6 \%$ & $17,8 \%$ & $34,6 \%$ & $32,0 \%$ & $11,9 \%$ \\
\hline Programmi televisivi & $28,9 \%$ & $21,9 \%$ & $25,8 \%$ & $14,4 \%$ & $9,0 \%$ \\
\hline Sistema socio-economico & $31,9 \%$ & $38,2 \%$ & $22,9 \%$ & $5,7 \%$ & $1,3 \%$ \\
\hline Sport & $5,2 \%$ & $15,8 \%$ & $39,5 \%$ & $24,4 \%$ & $15,1 \%$ \\
\hline Sistema politico & $58,4 \%$ & $23,0 \%$ & $12,9 \%$ & $4,1 \%$ & $1,6 \%$ \\
\hline Sistema sanitario & $19,9 \%$ & $30,7 \%$ & $35,7 \%$ & $10,1 \%$ & $3,6 \%$ \\
\hline Territorio e ambiente & $15,3 \%$ & $28,5 \%$ & $32,6 \%$ & $15,0 \%$ & $8,5 \%$ \\
\hline
\end{tabular}


55. Indica quanto sei d'accordo con le seguenti affermazioni:

\begin{tabular}{|c|c|c|c|c|}
\hline & $\begin{array}{c}\text { Molto } \\
\text { d'accordo }\end{array}$ & $\begin{array}{c}\text { Abbastanza } \\
\text { d'accordo }\end{array}$ & $\begin{array}{l}\text { Abbastanza } \\
\text { contrario }\end{array}$ & $\begin{array}{c}\text { Molto } \\
\text { contrario }\end{array}$ \\
\hline $\begin{array}{l}\text { La presenza di stranieri } \\
\text { in Italia è positiva perché } \\
\text { stimola il confronto tra la } \\
\text { nostra cultura e le altre }\end{array}$ & $32,0 \%$ & $50,3 \%$ & $14,4 \%$ & $3,4 \%$ \\
\hline $\begin{array}{l}\text { Gli stranieri danno un utile } \\
\text { contributo all'economia } \\
\text { del territorio }\end{array}$ & $19,6 \%$ & $47,2 \%$ & $25,8 \%$ & $7,5 \%$ \\
\hline $\begin{array}{l}\text { Gli stranieri sono persone } \\
\text { che cercano soltanto di } \\
\text { migliorare la loro condizione } \\
\text { di vita senza recar danno } \\
\text { a nessuno }\end{array}$ & $18,6 \%$ & $55,8 \%$ & $19,9 \%$ & $5,7 \%$ \\
\hline $\begin{array}{l}\text { Le amministrazioni locali } \\
\text { devono essere più attente } \\
\text { alle problematiche degli } \\
\text { stranieri per favorire la loro } \\
\text { integrazione }\end{array}$ & $29,5 \%$ & $47,7 \%$ & $18,7 \%$ & $4,1 \%$ \\
\hline $\begin{array}{l}\text { Gli stranieri portano via il } \\
\text { lavoro e le case agli italiani }\end{array}$ & $3,6 \%$ & $14,5 \%$ & $43,0 \%$ & $38,9 \%$ \\
\hline $\begin{array}{l}\text { Gli stranieri portano degrado } \\
\text { nei luoghi in cui viviamo }\end{array}$ & $4,1 \%$ & $16,0 \%$ & $43,2 \%$ & $36,7 \%$ \\
\hline
\end{tabular}

56. Conosci all'Aquila luoghi dedicati ad altre culture (ristoranti, alimentari, circoli e associazioni, luoghi di culto, luoghi di incontro, ecc.)?

$\square$ Sì $23,71 \%$
$\square$ No $76,29 \%$

Quali?

\section{Pensi di rimanere a vivere in Italia?}

$\square$ Sì, perché $33,8 \%$

$\square$ No, perché $13,1 \%$

$\square$ Non so, perché $53,1 \%$

58. Come valuti, su una scala da 1 a 5 , la città dell'Aquila rispetto ai seguenti aspetti?

\begin{tabular}{lrcccc}
\hline & 1 & 2 & 3 & 4 & 5 \\
\hline $\begin{array}{l}\text { Condizioni abitative generali } \\
\text { (qualità, costi e reperibilità } \\
\text { degli alloggi) }\end{array}$ & $20,4 \%$ & $36,6 \%$ & $33,8 \%$ & $7,7 \%$ & $1,5 \%$ \\
\hline Prospettive occupazionali & & & & & \\
\hline Servizi sociosanitari & $31,8 \%$ & $41,9 \%$ & $23,0 \%$ & $3,4 \%$ & $0,0 \%$ \\
\hline
\end{tabular}




\begin{tabular}{lrrrrr}
\hline Relazioni interpersonali e vita sociale & $14,5 \%$ & $25,6 \%$ & $39,0 \%$ & $18,1 \%$ & $2,8 \%$ \\
\hline $\begin{array}{l}\text { Servizi per il pubblico offerti da } \\
\text { uffici e istituzioni }\end{array}$ & $21,4 \%$ & $37,1 \%$ & $34,8 \%$ & $5,4 \%$ & $1,3 \%$ \\
\hline Intrattenimento e svago & $21,7 \%$ & $37,7 \%$ & $31,3 \%$ & $8,5 \%$ & $0,8 \%$ \\
\hline Stimoli culturali & $22,3 \%$ & $33,9 \%$ & $33,2 \%$ & $9,3 \%$ & $1,3 \%$ \\
\hline $\begin{array}{l}\text { Spazi per il tempo libero e luoghi } \\
\text { di aggregazione }\end{array}$ & $26,0 \%$ & $36,6 \%$ & $28,3 \%$ & $7,5 \%$ & $1,6 \%$ \\
\hline $\begin{array}{l}\text { Trasporti e mobilità sul territorio } \\
\text { urbano }\end{array}$ & $38,4 \%$ & $33,8 \%$ & $19,1 \%$ & $8,2 \%$ & $0,5 \%$ \\
\hline $\begin{array}{l}\text { Assetto urbano (nuovi agglomerati, } \\
\text { situazione del centro storico...) }\end{array}$ & $45,9 \%$ & $35,8 \%$ & $14,2 \%$ & $3,1 \%$ & $1,0 \%$ \\
\hline $\begin{array}{l}\text { Sicurezza } \\
\text { Qualità del paesaggio }\end{array}$ & $18,1 \%$ & $33,6 \%$ & $33,9 \%$ & $12,7 \%$ & $1,8 \%$ \\
\hline Qualità del territorio & $6,5 \%$ & $12,9 \%$ & $30,2 \%$ & $31,4 \%$ & $20,6 \%$ \\
\hline Vita politica & $29,8 \%$ & $36,6 \%$ & $28,7 \%$ & $4,2 \%$ & $0,8 \%$ \\
\hline Luoghi di culto e spiritualità & $11,1 \%$ & $22,6 \%$ & $45,3 \%$ & $16,3 \%$ & $4,7 \%$ \\
\hline Qualità della vita & $10,4 \%$ & $25,2 \%$ & $48,6 \%$ & $13,5 \%$ & $2,3 \%$ \\
\hline
\end{tabular}

59. Nella tua esperienza di studio all'Aquila, quanto ti senti solidale oggi rispetto alle vicende legate ai problemi della ricostruzione del tessuto urbano, sociale e culturale? (da un minimo di 1 a un massimo di 5)

\begin{tabular}{|c|c|c|c|c|}
\hline 1 & 2 & 3 & 4 & 5 \\
$3,4 \%$ & $10,1 \%$ & $27,6 \%$ & $26,8 \%$ & $32,2 \%$ \\
\hline
\end{tabular}

60. Vuoi aggiungere qualcosa?

GRAZIE PER LA COLLABORAZIONE! 


\section{Bibliografia}

M. Aime, Eccessi di culture, Einaudi, Torino, 2004.

M. Allevi, "Cultura e luoghi: quando l'abitare diventa atopico. Esempi da L'Aquila post sisma”, in: M. Pedrana (a cura di), Multiculturalità e territorializzazione. Casi di studio, IF Press, Roma, 2013, pp. 33-54.

M. Allevi, "La quotidianità aquilana stravolta", in: L.M. Calandra (a cura di), Territorio e democrazia. Un laboratorio di geografia sociale nel doposisma aquilano, L'Una, L'Aquila, 2012, pp. 125-137.

G.W. Allport, La natura del pregiudizio, tr. it.: La Nuova Italia, Firenze, 1973.

M. Ambrosini, Non passa lo straniero. Le politiche migratorie tra sovranità nazionale e diritti umani, Cittadella, Assisi, 2014.

M. Ambrosini e M. Caneva, "Les adolescents d'origine immigrée: processus d'identification entre liens familiaux et société d'accueil", Migrations Société, 141-142, mai-août 2012, pp. 119-139 tr. it.

U. Ascoli (a cura di), Il welfare mix in Europa, Carocci, Roma, 2003.

J. Atkinson, La motivazione, Il Mulino, Bologna, 1973.

A. Baldry e R. Ardone, Mediare $i$ conflitti a scuola. Presupposti teorici e intervento psicososociale, Carocci, Roma, 2003.

A. Bandura, Adolescenti e autoefficacia. Il ruolo delle credenze personali nello sviluppo individuale, tr. it. Erickson, Trento, 2012.

Z. Bauman, La solitudine del cittadino globale, tr. it. Feltrinelli, Milano, 2000.

T. Ben Jelloun, Il razzismo spiegato a mia figlia, tr. it. Bompiani, Milano, 1998.

G. Benvenuto (a cura di), La scuola diseguale. Dispersione ed equità nel sistema di istruzione e formazione, Anicia, Roma, 2011.

G. Benvenuto e P. Sposetti, "Valutare il parlato degli studenti universitari", in: E. Lugarini (a cura di), Valutare le competenze linguistiche, Atti del XV Convegno nazionale GISCEL, FrancoAngeli, Milano, 2010, pp. 405-415.

C. Bonifazi, L'Italia delle migrazioni, Il Mulino, Bologna, 2013.

P. Boscolo, La fatica e il piacere di imparare. Psicologia della motivazione scolastica, UTET, Novara, 2012.

P. Bourdieu, La distinzione, critica sociale del gusto, tr. it. Il Mulino, Bologna, 2001.

R.A. Bush Baruch e J. Folger, La promessa della mediazione, tr. it. Vallecchi, Firenze, 2009.

L.M. Calandra (a cura di), Territorio e democrazia. Un laboratorio di geografia sociale nel doposisma aquilano, L'Una, L'Aquila, 2012. 
M. Campanini e K. Merzan, Arcipelago Islam. Tradizione, riforma e militanza in età contemporanea, Laterza, Bari-Roma, 2007.

R. Canestrari e A. Godino, Trattato di psicologia, CLUEB, Bologna, 1997.

T. Caponio, Città italiane e immigrazione. Discorso pubblico e politiche a Milano, Bologna e Napoli, Il Mulino, Bologna, 2006.

M. Catarci, All'incrocio dei saperi. Una didattica per una società multiculturale, Anicia, Roma, 2004.

M. Catarci e M. Fiorucci, Orientamenti interculturali. Scelte scolastiche e opportunità sociali degli alunni con cittadinanza non italiana, Armando, Roma, 2013.

M. Catarci e M. Fiorucci, Intercultural Education in the European Context. Theories, Experiences, Challenges, Ashgate, London, 2015.

A. Colombo, Fuori controllo? Miti e realtà dell'immigrazione in Italia, Il Mulino, Bologna, 2012.

E. Colombo, "Le multiculturalisme quotidien des enfants d'immigrés en Italie", Migrations Société, 141-142, mai-août 2012, pp. 87-102.

A. Colombo, A. Genovese e A. Canevaro (a cura di), Immigrazione e nuove identità urbane. La città come luogo di incontro e scambio culturale, Erickson, Trento, 2006.

M. Colombo e V. Ongini (a cura di), Alunni con cittadinanza non italiana. L'eterogeneità dei percorsi scolastici - Rapporto 2012-2013, Quaderni ISMU, 1/2014, Fondazione ISMU, Milano, 2014.

Consiglio di Europa, Guida per lo sviluppo e l'attuazione di curricoli per un'educazione plurilingue e interculturale, Università degli Studi di Milano, Italiano LinguaDue, Semestrale del Master Promoitals, Supplemento n. 1, 2011 (http://riviste.unimi.it/index.php/promoitals/index/).

P. Coppola, “Territori di Allah", Terra d'Africa, III, 1994, pp. 9-14.

P. Corbetta, Metodologia e tecniche della ricerca sociale, Il Mulino, Bologna, 1999.

V. Cotesta, Sociologia dei conflitti etnici. Razzismo, immigrazione e società multiculturale, Laterza, Bari-Roma, 1999.

J. Cummins e M. Swain, Bilingualism in education: aspects of theory, research and practice, Longman, London-New York, 1986.

F. D'Ascenzo, "A Sud dell'immigrato. I congolesi tra Africa ed Europa", Meridione. Sud e Nord nel mondo, 2, aprile-giugno 2010, pp. 110-146.

A. Dal Lago, (a cura di), Lo straniero e il nemico. Materiali per l'etnografia contemporanea, Costa \& Nolan, Genova-Milano, 1998.

A. Dal Lago, Non persone. L'esclusione dei migranti in una società globale, Feltrinelli, Milano, 2004.

M. De Certeau, L'invenzione del quotidiano, Edizioni Lavoro, Roma, 2001.

M. de Donato e A. Stifano, "15 anni di sbarchi in Italia e nel Mediterraneo", in: IDOS, Dossier Statistico Immigrazione 2014 - Rapporto UNAR. Dalle discriminazioni ai diritti, IDOS, Roma, 2014, pp. 50-56.

E.L. Deci e R.M. Ryan, Intrinsic Motivation and Self-Determination in Human Behavior, Springer Science \& Business Media, New York, 1985.

E.L. Deci e R.M. Ryan, "Self-determination theory and the facilitation of intrinsic motivation, social development, and well-being", American Psychologist, 55, 2000, pp. 68-78. 
M. Delle Donne, Un cimitero chiamato Mediterraneo. Per una storia del diritto d'asilo nell'Unione Europea, DeriveApprodi, Roma, 2004.

EMN-Italia, L'immigrazione degli studenti internazionali in Italia, Roma, 2012.

Eurispes, $23^{\circ}$ Rapporto Italia 2011, Roma, Eurolink, 2011.

FAO, FIDA e PAM, L'état de l'insécurité alimentaire dans le monde 2013. Les multiples dimensions de la sécurité alimentaire, Fao, Roma, 2013.

C. Farvacque-Vitkovic, M. Raghunath, C. Eghoff e C. Boakye, Development of the Cities of Ghana. Challenges, Priorities and Tools, Africa Region Working Paper Series, Number 110, The World Bank, 2008 (www.worldbank.org/afr/ wps/wp110.pdf).

G. Favaro, I mediatori linguistici e culturali nella scuola, EMI, Bologna, 2001.

G. Favaro, Insegnare l'italiano agli alunni stranieri, La Nuova Italia, Firenze, 2002.

G. Favaro e E. Fumagalli, Capirsi diversi. Idee e pratiche di mediazione interculturale, Carocci, Roma, 2004.

L. Festinger, "A theory of social comparison processes", Human Relations, 7, 1954.

J. Finn e D. Finn, "Withdrawing From School”, Review of Educational Research, 59, 2, 1989, pp. 117-142.

M. Fiorucci, La mediazione culturale: strategie per l'incontro, Armando, Roma, 2000.

M. Fiorucci (a cura di), Incontri. Spazi e luoghi della mediazione interculturale, Armando, Roma, 2004.

M. Fiorucci (a cura di), Una scuola per tutti. Idee e proposte per una didattica interculturale delle discipline, FrancoAngeli, Milano, 2011.

M. Fiorucci, Gli altri siamo noi: la formazione interculturale degli operatori dell'educazione, Armando, Roma, 2011.

M. Foucault, Biopolitica e liberalismo, tr. it. Medusa, Milano, 2001.

Fondazione Migrantes, Rapporto Italiani nel Mondo 2014, Tau Editrice, Roma, 2014.

F. Frabboni e F. Pinto Minerva, Manuale di pedagogia generale, Laterza, BariRoma, 1999.

D. Francescato e E. Giusti, Empowerment e clinica, Edizioni Kappa, Roma, 1999.

L. Gaffuri, "I minori stranieri tra vantaggio demografico e rischi di marginalizzazione", in: IDOS, Dossier Statistico Immigrazione 2013 - Rapporto UNAR. Dalle discriminazioni ai diritti, IDOS, Roma, 2013, pp. 159-165.

L. Gaffuri, "Istituzione famigliare e unioni miste", in: IDOS, Dossier Statistico Immigrazione 2014 - Rapporto UNAR. Dalle discriminazioni ai diritti, IDOS, Roma, 2014, pp. 211-216.

U. Galimberti, Psicologia, Garzanti, Milano, 1999.

R. Galissot, M. Kilani e A. Rivera, L'imbroglio etnico in quattordici parolechiave, tr. it. Dedalo, Bari, 2001.

A. Genovese, Per una pedagogia interculturale. Dalla stereotipia dei pregiudizi all'impegno dell'incontro, Bononia University Press, Bologna, 2003.

Gfk-Eurisko, Gli atteggiamenti verso l'integrazione sociale degli stranieri, Rapporto di ricerca, Milano, 2010.

P. Ginsborg, Storia d'Italia dal dopoguerra a oggi, Einaudi, Torino, 1989.

IDOS, Dossier Statistico Immigrazione 2013 - Rapporto UNAR. Dalle discriminazioni ai diritti, IDOS, Roma, 2013.

IDOS, Dossier Statistico Immigrazione 2014 - Rapporto UNAR. Dalle discriminazioni ai diritti, IDOS, Roma, 2014. 
M.V. Isidori e A. Vaccarelli, Formazione e apprendimento in situazioni di emergenza e di post-emergenza, Armando, Roma, 2012.

K. Kornetis, "Una diaspora adriatica: la migrazione degli studenti universitari greci in Italia”, in: G. Minardi e E. Cocco (a cura di), Immaginare l'Adriatico, FrancoAngeli, Milano, 2007.

F. La Cecla, Mente locale. Per un'antropologia dell'abitare, Elèuthera, Milano, 1993.

M. Laeng, Pedagogia sperimentale, La Nuova Italia, Firenze, 1992.

F. Lagomarsino e A. Ravecca, Il passo seguente. I giovani di origine straniera all'università, FrancoAngeli, Milano, 2014.

F. Laudisa e A. Stanchi (a cura di), Alcuni dati sugli studenti stranieri iscritti nelle università piemontesi, Osservatorio per il Diritto allo Studio Universitario, 2004 (www.ossreg.piemonte.it/_library/downloadfile.asp?id=1955).

K. Lewin, I conflitti sociali. Saggi di dinamica di gruppo, tr. it. FrancoAngeli, Milano, 1972.

G. Li, L. Wang (ed.), Model Minority Myth Revisited: An Interdisciplinary Approach to Demystifing Asian american Educational Experience, Information Age, Charlotte (NC), 2008.

E. Lombardo, I dati statistici in pedagogia. Esplorazione e analisi, La Nuova Italia, Firenze, 1993.

L. Luatti, "Le rimesse nel 2013: segnali di ripresa a livello globale e in Italia", in: IDOS, Dossier Statistico Immigrazione 2014 - Rapporto UNAR. Dalle discriminazioni ai diritti, IDOS, Roma, 2014, pp. 26-33.

LUNARIA (a cura di), Cronache di ordinario razzismo. Terzo libro bianco sul razzismo in Italia, Lunaria, Roma, 2014 (www.cronachediordinariorazzismo.org).

A. Mariani (a cura di), 25 saggi di pedagogia, FrancoAngeli, Milano, 2011.

A.H. Maslow, Motivazione e Personalità, tr. it. Armando, Roma, 2010.

C. Midgley, M.L. Maehr, L.Z. Hruda e E. Anderman, Manual for the Patterns of Adaptive Learning Scales, University of Michigan, Michigan, 2000.

Ministero dell'Interno e IDOS (a cura di), Gli studenti internazionali nelle università italiane: indagine empirica e approfondimenti, Sesto Rapporto EMN-Italia, IDOS, Roma, 2013.

MIUR, Linee guida per l'accoglienza e l'integrazione degli alunni stranieri, Roma, 2006.

MIUR, Gli alunni stranieri nel sistema scolastico italiano. Anno scolastico 20112012, Ufficio di Statistica, 2012.

MIUR, Focus "Il passaggio dalla Scuola Secondaria di II grado all'Università". Diplomati Anno Scolastico 2011-2012 - Immatricolati Anno Accademico 20122013, Ufficio di Statistica, 2013a.

MIUR, Focus "La dispersione scolastica”, Ufficio di Statistica, Roma, 2013b.

MIUR, Linee guida per l'accoglienza e l'integrazione degli alunni stranieri, Roma, 2014.

MIUR e ISMU, Alunni con cittadinanza non italiana. L'eterogeneità dei percorsi scolastici. Rapporto nazionale Anno scolastico 2012-2013, Quaderni ISMU, 1/2014, Fondazione ISMU, Milano, 2014.

MIUR e ISMU, Alunni con cittadinanza non italiana. Tra difficoltà e successi. Rapporto nazionale Anno scolastico 2013-2014, Quaderni ISMU, 1/2015, Fondazione ISMU, Milano, 2015. 
T.B. Murdock, "The social context of risk: Status and motivational predictors of alienation in middle school", Journal of Educational Psychology, 91, 1999, pp. $62-75$.

T.B. Murdock e A. Miller, "Teachers as Sources of Middle School Students' Motivational Identity: Variable-Centered and Person-Centered Analytic Approaches", The Elementary School Journal, 103, 2003, pp. 383-399.

M.P. Nanni e F. Pittau (a cura di), Africa-Italia. Scenari migratori, IDOS, Roma, 2010.

C. Neri, Gruppo, Borla, Roma, 2003.

E. Nigris, I conflitti a scuola: la mediazione pedagogico-didattica, Mondadori, Milano, 2002.

A. Nobile, Il pregiudizio. Natura, fonti e modalità di risoluzione, La Scuola, Brescia, 2014.

OECD, Education at a Glance 2011, OECD Indicators, OECD Publishing, 2011.

OECD, "Indicator C4: Who studies abroad and where?", Education at a Glance 2014: OECD Indicators, OECD Publishing, Paris, 2014 (www.oecd.org/edu/ EAG2014-Indicator\%20C4\%20\%28eng\%29.pdf).

Osservatorio di Pavia, "Notiziabilità della sicurezza nei telegiornali in Italia e in Europa", in: Osservatorio Europeo sulla Sicurezza, La sicurezza in Italia $e$ in Europa. Significati, immagine e realtà. Indagine sulla rappresentazione sociale e mediatica della sicurezza in Italia, Francia, Germania, Gran Bretagna, Spagna. Report 1/2011, luglio 2011.

F. Palma, "Dimensioni dell'abitare dopo il sisma. L'Aquila tra territorialità, emergenza e C.A.S.E.", in: L.M. Calandra (a cura di), Territorio e democrazia. Un laboratorio di geografia sociale nel doposisma aquilano, L'Una, L'Aquila, 2012, pp. 109-124.

V. Papadimitriou, Greci in Italia: Una emigrazione intellettuale, 1997 (www. culturitalia.info/ARCHIVIO/siena/97_1/Papadi.htm).

A. Pelliccia, "Gli studenti greci”, in: Ministero dell'Interno e IDOS (a cura di), Gli studenti internazionali nelle università italiane: indagine empirica $e$ approfondimenti, Sesto Rapporto EMN-Italia, IDOS, Roma, 2013, pp. 187189.

L. Pépin, The history of European cooperation in education and training, European Commission, Luxembourg, 2006.

A. Portera, Manuale di pedagogia interculturale, Laterza, Bari-Roma, 2013.

L. Queirolo Palmas, “"Nous sommes ici!» Les enfants de l'immigration contre une postérité inopportune”, Migrations Société, 141-142, mai-août 2012, pp. 103-118.

A. Reffieuna, Le relazioni sociali in classe: il test sociometrico, Carocci, Roma, 2003.

V. Rosa, “L'empowerment e il suo sviluppo nell'ambito clinico e nella formazione: alcune proposte per l'addestramento all'auto-potenziamento", in: D. Francescato e E. Giusti (a cura di), Empowerment e clinica: integrazione di tecniche per l'autopotenziamento in psicologia clinica di comunità $e$ psicoterapia umanistica integrata, Edizioni Kappa, Roma, 1999, pp. 61-212.

C. Saraceno, N. Sartor e G. Sciortino, Stranieri e disuguali. Le disuguaglianze nei diritti e nelle condizioni di vita degli immigrati, Il Mulino, Bologna, 2013.

A. Sayad, L’immigrazione o i paradossi dell'alterità, tr. it. Ombre corte, Verona, 2008. 
J.H. Schumann, "The acculturation model for second language acquisition", in: R.C. Gingras (ed.), Second language acquisition and foreign language teaching, Center for Applied Linguistics, Arlington, 1978.

A. Spila, L'Aquila solidale: racconti del post-terremoto, in: R. Solnit, Un paradiso all'inferno, Fandango Libri, Roma, 2009, pp. 437-467.

F. Susi, L'educazione interculturale tra teoria e prassi, Corso di Perfezionamento in Educazione Interculturale, Università di Roma Tre, Roma, 1998.

F. Susi (a cura di), Come si è stretto il mondo. L'educazione interculturale in Italia e in Europa: teorie, esperienze e strumenti, Armando, Roma, 1999.

P. Tabet, La pelle giusta, Einaudi, Torino, 1997.

P.A. Taguieff, Il razzismo. Pregiudizi, teorie, comportamenti, tr. it. Raffaello Cortina, Milano, 1999.

P.A. Taguieff, La forza del pregiudizio. Saggio sul razzismo e sull'antirazzismo, tr. it. Il Mulino, Bologna, 1994.

Transatlatic Trends, Transatlantic Trends: Immigration. Key Findings 2010 (http://trends.gmfus.org/immigration/).

A. Tosi, Dalla madrelingua all'italiano. Lingue ed educazione linguistica nell'Italia multietnica, La Nuova Italia, Firenze, 1995.

Tuttoscuola, Dispersione nella scuola secondaria superiore, Dossier Tuttoscuola, Roma, 2013.

S. Ulivieri, (a cura di), L'educazione e i marginali. Storie, teorie, luoghi e tipologie dell'emarginazione, La Nuova Italia, Firenze, 1997.

S. Ulivieri, Educare al femminile, ETS, Pisa, 2001.

UN-DESA, World Population Prospects: The 2012 Revision, Dvd Edition, June 2013.

UNDP, Africa. Human Development Report 2012. Towards a Food Secure Future, Regional Bureau for Africa, New York, 2012.

UN-Habitat, The State of African Cities 2010. Governance, Inequality and Urban Land Markets, Nairobi (Kenya), 2010.

UN-Habitat, The State of African Cities 2014. Re-imagining sustainable urban transitions, Nairobi (Kenya), 2014.

UNICEF, La condizione dell'infanzia nel mondo 2012. Figli delle città, Valprinting, Nepi (Viterbo), 2012.

A. Vaccarelli, L'italiano e le lingue altre nella scuola multiculturale. Fattori culturali e psico-socio-pedagogici negli apprendimenti linguistici degli studenti immigrati, ETS, Pisa, 2001.

A. Vaccarelli, L'apprendimento e l'insegnamento dell'italiano come L2, Università degli Studi di RomaTre, Roma, 2003.

A. Vaccarelli, Dal razzismo al dialogo interculturale. Il ruolo dell'educazione negli scenari della contemporaneità, ETS, Pisa, 2008.

A. Vaccarelli (a cura di), Italiani e immigrati dopo il terremoto nel territorio aquilano. Rapporto di ricerca sui bisogni sociali, educativi e sullo stato di convivenza, RicostruireInsieme, L'Aquila, 2010.

A. Vaccarelli, "L'inserimento scolastico degli alunni di cittadinanza non italiana: storia, problemi e prospettive pedagogiche", in: G. Benvenuto (a cura di), La scuola diseguale. Dispersione ed equità nel sistema di istruzione e formazione, Anicia, Roma, 2011.

A. Vaccarelli, Migranti e italiani all'Aquila dopo il terremoto. Stato della convivenza e bisogni formativi, in: L.M. Calandra (a cura di), Territorio e 
democrazia. Un laboratorio di geografia sociale nel doposisma aquilano, L'Una, L'Aquila, 2012, pp. 165-183.

C. Wihtol de Wenden, Atlas mondial des migrations, Éditions Autrement, Paris, 2009.

L. Zanfrini, Sociologia della convivenza interetnica, Laterza, Bari-Roma, 2004a.

L. Zanfrini, Sociologia delle migrazioni, Laterza, Bari-Roma, 2004b.

D. Zoletto, Dall'intercultura ai contesti eterogenei. Presupposti e ambiti di ricerca pedagogica, FrancoAngeli, Milano, 2012. 


\section{Gli autori}

Mario Alaggio si è laureato in Ingegneria presso l'Università dell'Aquila. Ha iniziato la sua esperienza di volontariato con Amnesty International, di cui ancora fa parte, proseguendo la sua attività con l'ARCI. È attivo nell'associazionismo aquilano sui temi dei diritti umani, della promozione sociale e della pace. Attualmente è Presidente provinciale di ARCI servizio Civile e membro della Casa della Pace. Fa parte del Direttivo provinciale ARCI che rappresenta anche in seno al Coordinamento RicostruireInsieme.

Marta Allevi è laureata presso l'Università di L'Aquila con tesi in Geografia sul turismo sostenibile e cartografia. Si occupa di GIS (Geographic Information Systems) e collabora con il laboratorio Cartolab del Dipartimento di Scienze Umane dell'Aquila. Ha collaborato alla redazione dell'Atlante del turismo sostenibile in Africa (a cura di L.M. Calandra e A. Turco, FrancoAngeli, Milano, 2007) ed è autrice di diversi contributi, tra cui "La quotidianità stravolta", in L.M. Calandra (a cura di), Territorio e democrazia. Un laboratorio di geografia sociale nel doposisma aquilano (Ed. L'Una, L'Aquila, 2012); "Cultura e luoghi: quando l'abitare diventa atopico. Esempi da L'Aquila post-sisma”, in M. Pedrana (a cura di), Multiculturalità e territorializzazione. Casi di studio (IF Press, Roma, 2014). Nell'ambito del progetto "Unidiversità" ha collaborato come tutrice della convivenza.

Sergio Bontempelli è Presidente di Africa Insieme e di Straniamenti, due associazioni toscane attive nella tutela dei diritti dei migranti. Lavora come operatore degli sportelli di consulenza legale dei comuni ed è formatore e consulente di amministrazioni pubbliche e cooperative. È redattore del giornale on line Corriere delle Migrazioni, e ha al suo attivo numerose pubblicazioni su immigrazione, rifugiati e rom. Fa parte dell'équipe scientifica dei Libri Bianchi sul razzismo in Italia.

Serena Castellani è dottoranda in Geografia presso l'Università di Padova con un progetto di ricerca sulla resilienza e gli spazi pubblici in situazioni di post-disastro. Ha fatto parte della redazione della rivista Terra d'Africa, si occupa di cartografia, GIS (Geographic Information System) e telerilevamento. Nel 2010, l'Associazione Nazionale di Cartografia (AIC) premia il suo elaborato Un atlante 
dello Sviluppo Locale in Guinea Bissau e Brasile (in parte pubblicato sulla rivista dell'AIC). Dal 2005, collabora attivamente con il laboratorio Cartolab del Dipartimento di Scienze Umane dell'Università dell'Aquila. È autrice di diversi contribuiti sulla salvaguardia del patrimonio storico e paesaggistico e sull'analisi integrata del rischio grazie ad una collaborazione con l'ENEA di Bologna. La pubblicazione più recente è del 2014: "Participation as a Possible Strategy of Post-Disaster Resilience: Young People and Mobility in L'Aquila (Italy)" in L.M. Calandra, G. Forino, A. Porru (ed.), Multiple Geographical Perspectives on Hazards and Disasters (Valmar, Roma, 2014).

Valentina Ciaccio si è laureata in Scienze dell'Investigazione presso l'Università degli Studi dell'Aquila. Rappresentante degli studenti dal 2010 ed ex-Presidente del Consiglio Studentesco, si occupa di diritto universitario, attivismo sociale e del rapporto fra Ateneo e territorio. Nel progetto "Unidiversità" ha preso parte come operatrice di banca dati.

Chiara Ciccozzi si è laureata in Scienze giuridiche presso l'Università di Roma Tre e in Scienze Psicologiche applicate presso l'Università degli Studi dell'Aquila. Svolge progetti nelle scuole primarie volti a promuovere nei bambini comportamenti di cooperazione, collaborazione e sentimenti di solidarietà.

Maria Grazia Ferretti ha conseguito la laurea specialistica in Scienze dell'Educazione e della Formazione nella società complessa presso l'Università degli Studi dell'Aquila. Collabora con l'associazione Abruzzo Centro Internazionale Crocevia con vari interventi nell'ambito di progetti sulle tematiche interculturali. Ha collaborato alla stesura del testo "Non uno di meno. Approccio all'interculturalità nella scuola secondaria di II grado", a cura di Valentina Botti (Associazione Abruzzo Centro Internazionale Crocevia, 2009) e del Rapporto "Ricerca qualiquantitativa sulla condizione delle assistenti familiari immigrate presenti sul territorio della provincia dell'Aquila" a cura di L. Rapacchietta, A. Vaccarelli (Provincia dell'Aquila, 2008).

Arianna Fiorenza è laureanda in Scienze della Formazione Primaria presso l'Università degli Studi dell'Aquila. Ricopre cariche di rappresentanza degli studenti presso gli organi centrali dell'Ateneo dell'Aquila. È attenta da anni ai temi del sociale ed è socia della Comunità XXIV Luglio. Svolge progetti nelle scuole riguardanti i temi della cittadinanza attiva. Nel progetto "Unidiversità" ha preso parte come operatrice di banca dati.

Massimiliano Fiorucci è professore associato di Pedagogia interculturale e sociale presso il Dipartimento di Scienze della Formazione dell'Università Roma Tre. La sua ricerca guarda soprattutto al tema della mediazione culturale. Tra i suoi più recenti volumi ricordiamo: Per forza nomadi. Problemi, possibilità e limiti delle politiche di integrazione sociale per $i$ Rom e Sinti a Roma (Aemme Publishing, Roma, 2011); con M. Catarci (a cura di), Orientamenti interculturali. Scelte scolastiche e opportunità sociali degli alunni con cittadinanza non italiana (Armando, Roma, 2013); con M. Catarci (ed.), Intercultural Education in the European Context. Theories, Experiences, Challenges (Ashgate, London, 2015). 
Luigi Gaffuri insegna Geografia presso il Dipartimento di Scienze Umane dell'Università dell'Aquila. È stato redattore e ha fatto parte del comitato scientifico della rivista Terra d'Africa e della rivista di cultura, arte, tecnologia Tutto da capo. Da venticinque anni svolge ricerca africanistica, con missioni di studio sul terreno nella fascia tropicale, occupandosi anche di immigrati e rifugiati. È stato tra i componenti del comitato scientifico sia del Dossier statistico immigrazione promosso da IDOS sia del Rapporto sugli indici di inserimento territoriale degli immigrati in Italia patrocinato dal CNEL. Ha scritto e curato numerosi volumi, pubblicando oltre centocinquanta lavori su riviste italiane e internazionali o inclusi in opere collettive, senza trascurare l'attività pubblicistica su testate nazionali.

Roberto Manzi, dopo essersi diplomato come attore presso l'Accademia Nazionale d'Arte Drammatica "Silvio D'Amico" di Roma, ha conseguito la laurea in Scienze della Formazione e del Servizio Sociale presso l'Università dell'Aquila. Ad oggi alterna il lavoro di attore a studi e ricerche sul rapporto e le connessioni tra pedagogia e teatro. Nel Progetto "Unidiversità" ha lavorato come operatore di banca dati.

Farnaz Mirzapoor, dopo gli studi secondari in Iran, ha conseguito la laurea in Ingegneria presso l'Università dell'Aquila. Dopo 10 anni da progettista elettronica decide di dimettersi per poter lavorare nel sociale. Da allora ha seguito un corso di mediazione culturale della regione Abruzzo, conseguito un master universitario di I livello in "Politiche dell'Incontro e Mediazione Culturale in contesto migratorio" presso l'Università degli Studi di Roma Tre e l'abilitazione all'insegnamento dell'informatica. Fondatrice nel 2004 dell'associazione culturale "il Mondo in una Stanza", di cui è stata presidente fino al 2012, ha lavorato come mediatrice culturale in vari progetti. Attualmente è docente di informatica e titolare di un centro ricreativo culturale a Carsoli.

Francesco Marola ha conseguito la laurea magistrale in Filologia moderna presso l'Università dell'Aquila, con una tesi in letterature comparate. È attivista di diverse associazioni e movimenti impegnati nel campo del sociale, ricoprendo anche incarichi di responsabilità. Nel progetto "Unidiversità" ha assunto il ruolo di operatore di banca dati.

Maria Marronaro, psicologa e psicoterapeuta sistemico relazionale, svolge la sua attività privata a L'Aquila. Collabora con l'Associazione Koinonia Onlus e l'Associazione RicostruireInsieme nell'ambito di progetti legati all'integrazione culturale nel difficile contesto sociale aquilano. È co-autrice del lavoro "Il giro del mondo giocando. Come il mondo può essere svelato attraverso il gioco" in S. Nanni (a cura di), Educare senza confini (FrancoAngeli, Milano, 2015).

Francesca Palma è laureanda in Culture per la comunicazione presso l'Università dell'Aquila. Si occupa di GIS (Geographic Information System) e di analisi socio-territoriale. Collabora dal 2010 con il laboratorio Cartolab del Dipartimento di Scienze Umane dell'Aquila. È autrice di "Dimensioni dell'abitare dopo il sisma. L'Aquila tra territorialità, emergenza e C.A.S.E." e diverse elaborazioni grafiche e cartografiche del percorso espositivo "Per una geografia sociale del doposisma 
aquilano" pubblicati in L.M. Calandra (a cura di), Territorio e democrazia. Un laboratorio di geografia sociale nel doposisma aquilano (L'Una, L'Aquila, 2012). È co-autrice di varie carte tra le quali "Il Comune dell'Aquila dopo il sisma del 6 aprile 2009" (2011).

Jean Pierre Ndayambaje ha una laurea in Contabilità e Gestione aziendale (Accounting and Management c/o Université Nationale du Rwanda - 1987). Lavora presso il Centro per l'Impiego dell'Amministrazione Provinciale dell'Aquila. Attualmente è Presidente del Coordinamento RicostruireInsieme, operando attivamente nei processi di inclusione dopo il sisma che ha colpito L'Aquila nel 2009.

Sonia Pagnanelli è laureanda in Lettere - curriculum geografia presso il Dipartimento di Scienze Umane dell'Aquila. Dal 2010 collabora attivamente con il Laboratorio Cartolab del Dipartimento di Scienze Umane su temi di geografia sociale. Ha partecipato al progetto "Unidiversità" in qualità di operatrice di banca dati.

Ilaria Salvati si è laureata in Amministrazione, Economia e Finanza presso l'Università dell'Aquila e ha sempre mostrato uno spiccato interesse per l'intercultura. Il suo percorso di studi è stato arricchito da diverse partecipazioni in attività didattiche e di ricerca e da esperienze formative all'estero, negli Stati Uniti, in Sudafrica e in Francia. Nel progetto "Unidiversità" ha ricoperto il ruolo di operatrice di banca dati. Attualmente è titolare di una borsa di ricerca per l'economia internazionale presso l'ICE-Agenzia per la promozione all'estero e l'internazionalizzazione delle imprese italiane di Roma ed è responsabile di un tutorial course in International Industrial Organization presso l'Università degli Studi dell'Aquila.

Anna Tozzi afferisce al Dipartimento di Ingegneria e Scienze dell'Informazione e Matematica dell'Università dell'Aquila. La sua attività didattica e di ricerca, con numerose pubblicazioni di livello internazionale, si concentra sui temi della geometria e della matematica e delle loro applicazioni. Dal 2004 è pro-rettore per le Relazioni Internazionali ed è responsabile dell'internazionalizzazione dell'Università dell'Aquila.

Alessandro Vaccarelli è professore associato di Pedagogia generale e interculturale presso il Dipartimento di Scienze Umane dell'Aquila. È Presidente del Corso di laurea in Scienze della Formazione e del Servizio sociale. Da anni si occupa, nella didattica e nella ricerca, dei temi relativi all'inclusione scolastica degli studenti di cittadinanza non italiana, di pregiudizio e di educazione antirazzista. Nel contesto del post-terremoto aquilano, ha attivato studi, ricerche e interventi sull'educazione nelle emergenze e nel post-emergenza. Tra i suoi lavori, si segnalano: Dal razzismo al dialogo interculturale (ETS, Pisa, 2008); Razzismo. Prospettive pedagogiche per la descostruzione, in M. Catarci, E. Macinai, Le parole chiave della pedagogia interculturale (ETS, Pisa, 2015); con M.V. Isidori, Pedagogia dell'emergenza. Didattica nell'emergenza (FrancoAngeli, Milano, 2014). 


\section{La melagrana}

diretta da Graziella Favaro e Massimiliano Fiorucci

\section{Ultimi volumi pubblicati:}

Idee e metodi per l'intercultura

Massimiliano TAROzZI, Dall'intercultura alla giustizia sociale. Per un progetto pedagogico e politico di cittadinanza globale.

Davide Zoletto, Dall'intercultura ai contesti eterogenei. Presupposti teorici e ambiti di ricerca pedagogica.

MARCo CATARCI, L'integrazione dei rifugiati. Formazione e inclusione nelle rappresentazioni degli operatori sociali (disponibile anche in e-book).

IVANA BologneSI, Insieme per crescere. Scuola dell'infanzia e dialogo interculturale.

Duccio Demetrio, Graziella Favaro, Didattica interculturale. Nuovi sguardi, competenze, percorsi.

MASSIMILIANO FIORUCCI (a cura di), Una scuola per tutti. Idee e proposte per una didattica interculturale delle discipline.

MANILA FRANZINI, Formazione alle competenze interculturali nell'adozione internazionale (disponibile anche in e-book).

Monica Ferrari, Filippo Ledda, SapienssII. Strumento per l'Autovalutazione dei Processi Interculturali in Educazione nella Scuola Secondaria di Secondo Grado.

IVANA Bolognesi, AdRIANA Di Rienzo, Io non sono proprio straniero. Dalle parole dei bambini alla progettualità interculturale.

Raffaele Mantegazza, Manuale di pedagogia interculturale. Tracce, pratiche e politiche per l'educazione alla differenza.

PaOla D'IGNAZI, Ragazzi immigrati. L'esperienza scolastica degli adolescenti attraverso l'intervista biografica.

Graziella Favaro, Susanna Mantovani, Tullia Musatti (a cura di), Nello stesso nido. Famiglie e bambini stranieri nei servizi educativi.

Caritas Ambrosiana, Centro Come, a cura di Sabrina Ignazi, Monica Napoli, L'inserimento scolastico dei bambini rom e sinti.

\section{Esperienze e progetti per l'intercultura}

IVAna Bolognesi, Adriana Di Rienzo, Stefania Lorenzini, Anna Pileri, Di cultura in culture. Esperienze e percorsi interculturali nei nidi d'infanzia.

Rosita Deluigi (a cura di), Formazione professionale e intercultura. Sfide pedagogiche tra pratica e riflessività (disponibile anche in e-book).

Agostino Portera (a cura di), Competenze interculturali. Teoria e pratica nei settori scolastico-educativo, giuridico, aziendale, sanitario e della mediazione culturale (disponibile anche in e-book). 


\section{STUDIARE IN ITALIA}

Dopo anni di dibattito sulla presenza degli studenti di cittadinanza non italiana nel sistema scolastico, i tempi sono maturi per aprire le prospettive di studio e di intervento della pedagogia interculturale anche al mondo accademico. Avanguardia dello stesso multiculturalismo, con una presenza straniera che da sempre l'ha strutturalmente segnata, seppur esigua al confronto con altri Paesi, l'università si trova oggi a rispondere a nuove sfide, che partono da una ridefinizione, quantitativa e qualitativa, della popolazione studentesca di cittadinanza non italiana: accanto alla figura più classica dello studente internazionale, che sceglie l'Italia come meta dei suoi studi e che vi arriva ponendo questo come suo obiettivo prioritario, si afferma oggi la presenza di quegli studenti, figli delle migrazioni, che si affacciano al mondo accademico dopo un'esperienza di scolarizzazione e di inserimento sociale non sempre semplice e lineare. Il volume, oltre a inquadrare gli scenari generali riferiti alla società e alla città multiculturale, all'internazionalizzazione degli studi, documenta un lavoro di ricerca pedagogica, con aperture anche alle scienze sociali e geografiche, svolto nell'Università dell'Aquila. L'indagine sul campo ha mirato a ricostruire i background, i percorsi accademici, i bisogni formativi, i problemi dell'inserimento sociale degli studenti internazionali e degli studenti stranieri scolarizzati in Italia, prestando particolare attenzione anche ai temi della socialità, della reciprocità, del pregiudizio e dell'inclusione anche in riferimento agli studenti italiani. La ricerca, ma anche l'azione: L'Aquila, una città universitaria ancora segnata dal sisma del 2009 e dall'alterazione dei rapporti sociali. Nel volume si riportano la descrizione e i principali risultati del progetto "Unidiversità" (FEI, annualità 2013, Azione 7, PROG-1059381), all'interno del quale si è puntato alla formazione di una nuova figura professionale, il tutore della convivenza, e alla sua implementazione in ambito accademico e urbano. Il libro si rivolge agli studiosi, agli amministratori, al mondo del volontariato e, naturalmente, agli studenti, per offrire un contributo tanto nell'ottica della conoscenza di una realtà ancora poco studiata, quanto come riferimento per la costruzione di buone prassi.

Alessandro Vaccarelli è professore associato di Pedagogia generale e interculturale presso il Dipartimento di Scienze Umane dell'Aquila e presidente del Corso di laurea in Scienze della Formazione e del Servizio sociale. Da anni si occupa dei temi relativi all'inclusione scolastica degli studenti di cittadinanza non italiana, di pregiudizio e di educazione antirazzista. Tra i suoi lavori si segnalano: Dal razzismo al dialogo interculturale (ETS, Pisa, 2008); Razzismo. Prospettive pedagogiche per la descostruzione, in M. Catarci, E. Macinai, Le parole chiave della pedagogia interculturale (ETS, Pisa, 2015); con M.V. Isidori, Pedagogia dell'emergenza. Didattica nell'emergenza (FrancoAngeli, Milano, 2014).

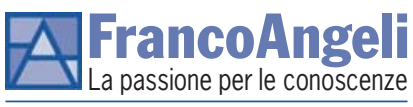

